\title{
Mathematical Analysis of Vortex Sheets
}

\author{
SIJUE WU \\ University of Michigan
}

\begin{abstract}
We consider the motion of the interface separating two domains of the same fluid that moves with different velocities along the tangential direction of the interface. The evolution of the interface (the vortex sheet) is governed by the Birkhoff-Rott (BR) equations. We consider the question of the weakest possible assumptions such that the Birkhoff-Rott equation makes sense. This leads us to introduce chord-arc curves to this problem. We present three results. The first can be stated as the following: Assume that the Birkhoff-Rott equation has a solution in a weak sense and that the vortex strength is bounded away from 0 and $\infty$. Moreover, assume that the solution gives rise to a vortex sheet curve that is chord-arc. Then the curve is automatically smooth, in fact analytic, for fixed time. The second and third results demonstrate that the Birkhoff-Rott equation can be solved if and only if only half the initial data is given. (C) 2005 Wiley Periodicals, Inc.
\end{abstract}

\section{Introduction}

Vortex dynamics is of fundamental importance for a wide variety of concrete physical problems, such as lift of airfoils, mixing of fluids, separation of boundary layers, and generation of sounds. In mathematical analysis, one often neglects surface tension and viscosity when they are small in the real physical problem. This necessitates justifying such simplifications.

In this paper, we consider the motion of the interface separating two domains of the same fluid in $\mathbb{R}^{2}$ that moves with different velocities along the tangential direction of the interface. We assume that the fluids occupying the two domains separated by the interface are of constant densities that are equal and inviscid, incompressible, and irrotational. We also assume that the surface tension is 0 , and that there are no external forces. The interface in the aforementioned fluid motion is a so-called vortex sheet. We want to study the following problem:

Given vortex sheet initial data, is there a unique solution to the problem?

In general, there are two approaches to the aforementioned problem. One is to solve the initial value problem of the incompressible Euler equation in $\mathbb{R}^{2}$ :

$$
\left\{\begin{array}{l}
\mathfrak{v}_{t}+\mathfrak{v} \cdot \nabla \mathfrak{v}+\nabla p=0 \\
\operatorname{div} \mathfrak{v}=0 \\
\mathfrak{v}(x, y, 0)=\mathfrak{v}_{0}(x, y)
\end{array} \quad(x, y) \in \mathbb{R}^{2}, t \geq 0\right.
$$


where the initial incompressible velocity $\mathfrak{v}_{0} \in L_{\text {loc }}^{2}\left(\mathbb{R}^{2}\right)$, in which the vorticity $\omega_{0}=$ curl $\mathfrak{v}_{0}$ is a finite Radon measure. Here $\mathfrak{v}$ is the fluid velocity, $p$ is the pressure, and the density of the fluid is assumed to be 1 . Notice that a vortex sheet gives a measure-valued vorticity supported on the interface. This approach was posed by DiPerna and Majda in 1987 [21]. In 1991, Delort [20] proved the existence of weak solutions global in time of the two-dimensional incompressible Euler equation (1.1) for measure-valued initial vorticity in $H_{\mathrm{loc}}^{-1}\left(\mathbb{R}^{2}\right)$ that has a distinguished sign. However, the problem of uniqueness of the weak solution is still unresolved.

In 1963, Yudovich [51] obtained the existence and uniqueness of weak solutions of the two-dimensional incompressible Euler equation (1.1) for initially bounded vorticity. The best results on uniqueness to date are given by Yudovich [52] and Vishik [49] for weak solutions with vorticity in a class slightly larger than $L^{\infty}$. This does not include vortex sheets, which admit measure-valued vorticity. Examples of weak solutions with the velocity field $\mathfrak{v} \in L^{2}\left(\mathbb{R}^{2} \times(-T, T)\right)$ that is compactly supported in space-time was constructed by Scheffer [44] and later by Shnirelman [45]. This gives nonuniqueness of weak solutions in $L^{2}\left(\mathbb{R}^{2} \times(-T, T)\right)$. However, nonuniqueness in the physically relevant class of conserved energy $\mathfrak{v} \in$ $L^{\infty}\left([0, \infty), L_{\text {loc }}^{2}\left(\mathbb{R}^{2}\right)\right)$ remains open. Sources containing numerical evidence of nonuniqueness of weak solutions for vortex sheet data include [33, 40].

Furthermore, weak solutions give little information on the specific nature of the vortex sheet evolution. For instance, does the vorticity remain supported on a curve for a later time given that the initial vorticity is supported on a curve in $\mathbb{R}^{2}$ ? If we further assume that the free interface between the two fluid domains remains a curve in $\mathbb{R}^{2}$ at a later time, equation (1.1) can be reduced to an evolutionary differential-integral equation along the interface. This is the Birkhoff-Rott equation, written explicitly by Birkhoff [2] and implied in the work of Rott [42]. The second approach uses the Birkhoff-Rott equation as a model for the evolution of the vortex sheet.

\subsection{The Birkhoff-Rott Equation}

For convenience, we use the complex variable $z=x+i y$ to denote a point in $\mathbb{R}^{2} \cdot \bar{z}=x-i y$ denotes the complex conjugate and $f_{t}=\partial_{t} f$ and $f_{x}=\partial_{x} f$ are the partial derivatives of the function $f . H^{s}(\mathbb{R})$ indicates a Sobolev space.

In search of the equation for the evolution of the vortex sheet, we suppose that at time $t \geq 0$ the vorticity is a measure supported on the curve $\Gamma(t)$ given by complex position $\xi=\xi(s, t)$ in arc length $s$, in which $\xi(0, t)$ is the particle path of a reference particle; on this curve the vorticity density is $\gamma=\gamma(s, t)$. That is, the vorticity at time $t$ is $\omega(x, y, t)$ satisfying

$$
\iint \phi(x, y) \omega(x, y, t) d x d y=\int \phi(\xi(s, t)) \gamma(s, t) d s \quad \text { for any } \phi \in C_{0}^{\infty}\left(\mathbb{R}^{2}\right) .
$$


From the Biot-Savart law, the velocity field $\mathfrak{v}$ induced by the vorticity is given by

$$
\overline{\mathfrak{v}}(z, t)=\frac{1}{2 \pi i} \int \frac{\gamma\left(s^{\prime}, t\right)}{z-\xi\left(s^{\prime}, t\right)} d s^{\prime} \quad \text { for } z \notin \Gamma(t) .
$$

Notice that the velocity is discontinuous just on $\Gamma(t)$. We define the velocity on the sheet as the average of the velocities at the two sides of the sheet that is given by the principal value integral:

$$
\overline{\mathfrak{v}}(\xi(s, t), t)=\frac{1}{2 \pi i} \mathrm{p} \cdot \mathrm{v} \cdot \int \frac{\gamma\left(s^{\prime}, t\right)}{\xi(s, t)-\xi\left(s^{\prime}, t\right)} d s^{\prime} .
$$

As suggested by the properties of the Euler equation, we assume that the vortex sheet is convected by the average velocity (1.2), and the vorticity is conserved along the particle path. We arrive at the evolution equation of the vortex sheet:

$$
\begin{aligned}
\xi_{t}(s, t)+a(s, t) \xi_{s}(s, t) & =\mathfrak{v}(\xi(s, t), t), \\
\gamma_{t}(s, t)+\partial_{s}(a(s, t) \gamma(s, t)) & =0,
\end{aligned}
$$

where $a(s, t)$ is a real-valued function satisfying $a(0, t)=0$.

A rigorous justification of the equivalence between equation (1.3) and equation (1.1) for smooth graphs $\xi(s, t)$ and smooth vortex strength $\gamma(s, t)$ can be found in [35]. Recently it was shown that equations (1.3) and (1.1) are equivalent under the assumptions that $\xi(\cdot, t)$ is a regular curve (see the paragraph following Theorem 1.1 for the definition of a regular curve) and $\gamma(\cdot, t) \in L^{2}(d s) \cap L^{1}(d s)$ (see [34, theorem 3.2]).

Assuming $\alpha(s, t)=\int_{0}^{s} \gamma\left(s^{\prime}, t\right) d s^{\prime}$ defines an increasing function of $s$, we make a change of variables: $z(\alpha, t)=\xi(s(\alpha, t), t)$, in which $s(\cdot, t)$ is the inverse of $\alpha(\cdot, t): \alpha(s(\alpha, t), t)=\alpha$. We get from equation (1.3) the Birkhoff-Rott equation

$$
\partial_{t} \bar{z}(\alpha, t)=\frac{1}{2 \pi i} \mathrm{p} \cdot \mathrm{v} \cdot \int \frac{1}{z(\alpha, t)-z(\beta, t)} d \beta .
$$

Notice that $z=z(\alpha, t)$ is a parametrization of the vortex sheet in the circulation variable $\alpha$, and $1 /\left|z_{\alpha}\right|=\gamma$ is the vortex strength. A steady solution of (1.4) is the flat sheet $z=\alpha$.

Equation (1.4) has been under active investigation for the last four decades. A well-known property of (1.4) is that perturbations of the flat sheet grow due to the Kelvin-Helmholtz instability, following from a linearization of equation (1.4) about the flat sheet. For given analytic data, Sulem, Sulem, Bardos, and Frisch [47] established the short-time existence and uniqueness of solutions in the analytic class for two-dimensional and three-dimensional vortex sheet evolution. Duchon and Robert [22] obtained the global existence of solutions of equation (1.4) for a special class of initial data that is close to the flat sheet.

However, numerous results show that a vortex sheet can develop curvature singularities in finite time from analytic data. Moore [37] was the first to provide analytical evidence that predicts the occurrence and time of singularity formation, which was verified numerically by Meiron, Baker, and Orszag [36] and by 
Krasny [28]. Caflisch and Orellana [3] proved existence almost up to the time of expected singularity formation for analytic data that is close to the flat sheet. Duchon and Robert [22] and Caflisch and Orellana [4] constructed specific examples of solutions of equation (1.4) where a curvature singularity develops in finite time from analytic data. The example of Caflisch and Orellana [4] has the form $z(\alpha, t)=\alpha+S(\alpha, t)+r(\alpha, t)$, where

$$
S(\alpha, t)=\epsilon(1-i)\left\{\left(1-e^{-t / 2-i \alpha}\right)^{1+\mu}-\left(1-e^{-t / 2+i \alpha}\right)^{1+\mu}\right\}
$$

is a solution of the linearized equation in which $\epsilon$ is small, $\mu>0 ; r(\alpha, t)$ is the correction term that is negligible relative to $S(\alpha, t)$ in the sense that $S(\alpha, t)+r(\alpha, t)$ exhibits the same kind of behavior as $S(\alpha, t)$ [4]. Notice that $S(\alpha, t)$ is an analytic function for $t>0$, but $S(\alpha, 0)$ has an infinite second derivative at $\alpha=0$ for $\mu \in(0,1)$. In fact, the $(1+v)^{\text {th }}$ derivative of $S(\alpha, 0)$ for $v>\mu$ becomes infinite at $\alpha=0$. Now inverting time gives an example $\hat{z}(\alpha, t)$ that is analytic at $t_{0}<0$ but has an infinite second derivative at $\alpha=0, t=0$. At the singularity formation time $t=0$, the vortex strength $1 /\left|\hat{z}_{\alpha}\right|$ of this example satisfies

$$
0<c \leq \frac{1}{\left|\hat{z}_{\alpha}\right|} \leq C<\infty
$$

for some constants $c$ and $C$, and $\hat{z}(\alpha, t) \in C^{1+\rho}\left(\mathbb{R} \times\left[t_{0}, 0\right]\right)$ for $0<\rho<\mu$.

These examples also show that the initial value problem of the Birkhoff-Rott equation (1.4) is ill-posed in $C^{1+v}(\mathbb{R}), v>0$, and in Sobolev spaces $H^{s}(\mathbb{R}), s>\frac{3}{2}$, in the Hadamard sense [4, 22]. Ill-posedness was also proved by Ebin [23] using a different approach. However, the existence of solutions in spaces less regular than $C^{1+v}(\mathbb{R})$ or $H^{s}(\mathbb{R})$ and the nature of the vortex sheet at and beyond the singularity time remained unknown analytically in general.

This suggests that we look for solutions of the Birkhoff-Rott equation in the largest possible spaces where the equation makes sense. For the purpose of this paper, we consider functions $z(\alpha, t)$ so that both sides of equation (1.4) are functions

locally in $L^{2}$, and on which the $L^{2}$ analysis is available. This leads us to consider chord-arc curves.

Another reason that chord-arc curves are to be considered is due to physical and numerical evidence. Krasny [27, 29] studied the evolution of the vortex sheet beyond singularity using numerical methods (more specifically, the vortex blob method). He found that the approximate solutions have the form of an infinitely rolled-up spiral beyond singularity. We know a special example of chord-arc curves is a logarithmic spiral. Physical and experimental evidence also suggests that vortex sheets are spirals (see [48]).

\subsection{Chord-Arc Curves and Some Recent Results}

Let $\Gamma$ be a rectifiable Jordan curve in $\mathbb{R}^{2}$ given by $\xi=\xi(s)$ in the arc length $s$. We say $\Gamma$ is a chord-arc curve if there is a constant $M \geq 1$ such that

$$
\left|s_{1}-s_{2}\right| \leq M\left|\xi\left(s_{1}\right)-\xi\left(s_{2}\right)\right| \text { for all } s_{1}, s_{2} .
$$


The infimum of all such constants $M$ is called the chord-arc constant.

For $Q \subset \mathbb{R}$ interval, let

$$
m_{Q}(f)=\frac{1}{|Q|} \int_{Q} f(x) d x .
$$

Let $I \subset \mathbb{R}$ be an interval. We say a function $f \in L_{\text {loc }}^{1}(I)$ is in $\operatorname{BMO}(I)$ if

$$
\|f\|_{\mathrm{BMO}(I)}=\sup _{\substack{Q \subset I \\ Q \text { interval }}} m_{Q}\left(\left|f-m_{Q} f\right|\right)<\infty .
$$

For a chord-arc curve $\xi=\xi(s), s$ the arc length, it is proved in [15] that $\xi^{\prime}(s)$ exists almost everywhere, and there is a choice of the argument function $b \in \mathrm{BMO}$, with $\xi^{\prime}(s)=e^{i b(s)}$. In particular, if the chord-arc constant is close to 1 , there is a choice of $b \in \mathrm{BMO}$ such that $\|b\|_{\mathrm{BMO}}$ is close to 0 . Moreover, the subset of all such functions $b$ is an open subset of BMO. And if $b \in \mathrm{BMO}$ and $\|b\|_{\mathrm{BMO}}<1$, $\xi(s)=\xi_{0}+\int_{0}^{s} e^{i b\left(s^{\prime}\right)} d s^{\prime}$ defines a chord-arc curve, with chord-arc constant $\leq$ $1 /\left(1-\|b\|_{\text {ВMO }}\right)$.

Examples of chord-arc curves include Lipschitz curves and logarithmic spirals $r= \pm e^{\theta}, \theta \in R$, where $(r, \theta)$ are the polar coordinates.

A theorem of David [17] states the following:

THEOREM 1.1 For all chord-arc curves $\Gamma: \xi=\xi(s)$, s the arc length, the corresponding Cauchy integral operator $\mathcal{C}_{\Gamma}$, where

$$
\mathcal{C}_{\Gamma} f(s)=\mathrm{p} . \mathrm{v} \cdot \int \frac{f\left(s^{\prime}\right)}{\xi(s)-\xi\left(s^{\prime}\right)} d \xi\left(s^{\prime}\right)
$$

is bounded from $L^{2}(d s)$ to $L^{2}(d s)$.

In fact, the result in [17] is stronger than stated above. David proved that the Cauchy integral operator $\mathcal{C}_{\Gamma}$ is bounded from $L^{2}(d s)$ to $L^{2}(d s)$ if and only if $\Gamma$ is a regular curve. A rectifiable curve $\Gamma$ is said to be regular if there is a constant $M$ such that for every $r>0$ and every disc $D$ with radius $r$, the length of $\Gamma \cap D$ does not exceed $M r$. A chord-arc curve is regular but not vice versa. We choose to work in the class of chord-arc curves instead of regular curves because for chordarc curves, there is a description in terms of BMO functions, and the chord-arc class is only slightly smaller than the regular class.

Now we go back to the Birkhoff-Rott equation. Notice that the Biot-Savart integral representing an incompressible velocity field $\mathfrak{v}$ in terms of the vorticity $\omega$ can be divergent if $\omega$ does not vanish fast enough at infinity, even if the velocity field $\mathfrak{v}$ is well-defined. We extend the definition of the Birkhoff-Rott equation (1.4) 
by considering the differences of the velocities between any two points,

$$
\begin{aligned}
\overline{z_{t}}(\alpha, t)-\overline{z_{t}}\left(\alpha^{\prime}, t\right) \\
=\frac{1}{2 \pi i} \mathrm{p} \cdot \mathrm{v} \cdot \int_{|\beta| \leq N}\left\{\frac{1}{z(\alpha, t)-z(\beta, t)}-\frac{1}{z\left(\alpha^{\prime}, t\right)-z(\beta, t)}\right\} d \beta \\
\quad+\frac{1}{2 \pi i} \mathrm{p} \cdot \mathrm{v} \cdot \int_{|\beta|>N} \frac{z\left(\alpha^{\prime}, t\right)-z(\alpha, t)}{(z(\alpha, t)-z(\beta, t))\left(z\left(\alpha^{\prime}, t\right)-z(\beta, t)\right)} d \beta
\end{aligned}
$$

for all $(\alpha, t)$ and $\left(\alpha^{\prime}, t\right)$, and some $N>|\alpha|+\left|\alpha^{\prime}\right|+1$. This admits a larger class of solutions. In particular, the integral on the right-hand side of equation (1.6) is convergent for those similarity solutions considered in [26, 39, 40, 41], which otherwise give divergent Cauchy integrals in (1.4) due to the divergent contributions from the vorticities at infinity. It follows from the theorem of David that the integral on the right-hand side of equation (1.6) is convergent for a.e. $(\alpha, t)$ and $\left(\alpha^{\prime}, t\right)$ and is in $L^{\infty}\left([0, T], L_{\mathrm{loc}}^{2}(d \alpha) \times L_{\mathrm{loc}}^{2}\left(d \alpha^{\prime}\right)\right)$ for the solutions considered in Theorem 1.2 in the following.

Notice that equation (1.6) determines a solution $z=z(\alpha, t)$ up to translations. That is, if $z=z(\alpha, t)$ is a solution of (1.6), then for any function $c(t), z(\alpha, t)+c(t)$ is also a solution of (1.6).

Roughly speaking, if a function $z=z(\alpha, t)$ satisfies equation (1.4), it also satisfies equation (1.6). On the other hand, if $z=z(\alpha, t)$ satisfies (1.6), and the Cauchy integral

$$
\frac{1}{2 \pi i} \mathrm{p} . \mathrm{v} \cdot \int \frac{1}{z(\alpha, t)-z(\beta, t)} d \beta
$$

is convergent at some point $\alpha$, say at $\alpha=0$, then there is a function $c=c(t)$ such that $z(\alpha, t)+c(t)$ satisfies equation (1.4). The function $c(t)$ is determined by

$$
\overline{\partial_{t}(z(0, t)+c(t))}=\frac{1}{2 \pi i} \text { p. v. } \int \frac{1}{z(0, t)-z(\beta, t)} d \beta .
$$

For $f \in L_{\text {loc }}^{1}(I), I \subset \mathbb{R}$ interval, we say $f$ is of bounded local mean oscillation on $I$ if there exists $\delta_{0}>0$ such that

$$
\|f\|_{\mathrm{BMO}(I), \delta_{0}}=\sup _{\substack{\text { all } Q \subset I \\|Q| \leq \delta_{0}}} \frac{1}{|Q|} \int_{Q}\left|f(\alpha)-m_{Q}(f)\right| d \alpha<\infty ;
$$

here $Q$ is an interval. We say $f$ is analytic on $I$ if $f \in C^{\infty}(I)$, and for any compact subset $K$ of $I$, there is a constant $\rho>0$ such that

$$
\sum_{m=o}^{\infty} \frac{\rho^{m}}{m !}\left(\int_{K}\left|\partial_{\alpha}^{m} f(\alpha)\right|^{2} d \alpha\right)^{1 / 2}<\infty
$$

Notice that $\ln z$ is multivalued for a complex number $z$. In the following, $\ln z_{\alpha}$ refers to one choice of the multivalues. We have the following results concerning the solutions of the Birkhoff-Rott equation (1.6) (or (1.4)): 
TheOREM 1.2 Assume that $z \in H^{1}\left([0, T], L_{\text {loc }}^{2}(\mathbb{R})\right) \cap L^{2}\left([0, T], H_{\text {loc }}^{1}(\mathbb{R})\right)$ and that $z$ is a solution of the Birkhoff-Rott equation (1.6) for $0 \leq t \leq T$ satisfying the following:

(i) There are constants $m>0$ and $M>0$, independent of $t$, such that

(1.7) $m|\alpha-\beta| \leq|z(\alpha, t)-z(\beta, t)| \leq M|\alpha-\beta|$ for all $\alpha, \beta \in R, 0 \leq t \leq T$.

Then there is a constant $c(m, M)>0$ as follows: if also

(ii) on some fixed interval $(a, b)$, there exist a determination of $\ln z_{\alpha}$ and $a$ constant $\delta_{0}>0$, independent of $t$, satisfying

$$
\sup _{[0, T]}\left\|\ln z_{\alpha}(\cdot, t)\right\|_{\mathrm{BMO}(a, b), \delta_{0}} \leq c(m, M),
$$

then $z_{\alpha} \in C((a, b) \times(0, T))$, and for each $t_{0} \in(0, T), z_{\alpha}\left(\cdot, t_{0}\right)$ is analytic on $(a, b)$.

Remark 1.3. Notice that assumption (ii) is satisfied if $\ln z_{\alpha} \in C([a, b] \times[0, T])$. Assumption (i) is equivalent to assuming that the vortex strength $\gamma=1 /\left|z_{\alpha}\right|$ is bounded away from 0 and $\infty$, with bounds independent of $t$, and $z=z(\cdot, t)$ defines a chord-arc curve for each fixed $t \in[0, T]$, with the chord-arc constant independent of $t$. Heuristically, assumption (i) is equivalent to assuming that the chord-arc curve $z=z(\cdot, t)$ "doesn't roll up too fast locally on the section $(a, b)$." Assumption (i) can be relaxed by requiring that $1 /\left|z_{\alpha}(\alpha, t)\right|$ be bounded away from 0 and $\infty$ for $\alpha \in(a, b)$ only and by assuming some weaker conditions for $\alpha \notin$ $(a, b)$.

A result similar to Theorem 1.2 under the stronger assumptions that the solution of the Birkhoff-Rott equation (1.4) $z=z(\alpha, t) \in C^{1+\rho_{0}}$ for some $\rho_{0}>0$, the vortex strength $c_{0}<\gamma(\alpha, t)<C_{0}$ for some constants $c_{0}>0$ and $C_{0}>0$, and the vortex sheets $\Gamma(t)$ are closed Jordan curves (it is easy to check that such curves are chordarc) is also obtained by Lebeau [31] using an independent approach. We'd like to point out here that the result of Lebeau doesn't aim at answering the question of the nature of the vortex sheet after singularity formation, since $C^{1+\rho_{0}}$ is a class that doesn't include any spiral curves; moreover, the assumption that the solution is $C^{1+\rho_{0}}$ in time also seems too strong.

As a consequence of Theorem 1.2, we know that the example constructed by Caflisch and Orellana [4] will fail to satisfy properties (i) and (ii) as stated in Theorem 1.2 near the singularity after the singularity formation time. That is, after the singularity formation time, the vortex sheet will fail to be in the class of chord-arc curves that don't roll up too fast near the singularity.

We now consider the question of existence of solutions of the Birkhoff-Rott equation for given initial data. What would constitute an initial datum for the Birkhoff-Rott equation? Notice that for the incompressible Euler equation (1.1), the initial datum is the initial velocity $\mathfrak{v}(\cdot, 0)$, which determines the initial vorticity $\omega_{0}=\operatorname{curl} \mathfrak{v}(\cdot, 0)$, and vice versa through the Biot-Savart law. For a vortex sheet datum, the initial vorticity $\omega_{0}$ is a Radon measure supported on a curve, which is 
determined if and only if both the initial position $\xi(\cdot, 0)$ of the vortex sheet and the initial vortex strength $\gamma(\cdot, 0)$ are (independently) given. We know $z(\alpha, 0)$ is the reparametrization of the vortex sheet in the circulation variable $\alpha$, with vortex strength $\gamma=1 /\left|z_{\alpha}\right|$. Therefore equivalent to the initial velocity $\mathfrak{v}(\cdot, 0)$ for the Euler equation is the initial datum $z(\cdot, 0)$ for the Birkhoff-Rott equation.

Let $\operatorname{Re} z$ and $\operatorname{Im} z$ be the real and imaginary parts of a complex number $z$, respectively. Regarding the existence of solutions, we have the following:

THEOREM 1.4 For any real-valued function $w_{0} \in H^{3 / 2}(\mathbb{R})$, there exists $T=$ $T\left(\left\|w_{0}\right\|_{H^{3 / 2}}\right)>0$ such that the Birkhoff-Rott equation (1.6) has a solution $z=$ $z(\alpha, t)$ for $0 \leq t \leq T$ satisfying $\ln z_{\alpha} \in C\left([0, T], H^{3 / 2}(\mathbb{R})\right) \cap C^{1}\left([0, T], H^{1 / 2}(\mathbb{R})\right)$ and $\operatorname{Im}\left\{(1+i) \ln z_{\alpha}(\alpha, 0)\right\}=w_{0}(\alpha)$, with the property that there exist constants $m>0$ and $M>0$ independent of $t$ such that

$$
m|\alpha-\beta| \leq|z(\alpha, t)-z(\beta, t)| \leq M|\alpha-\beta| \text { for all } \alpha, \beta \in \mathbb{R}, 0 \leq t \leq T .
$$

Notice that a consequence of $\ln z_{\alpha} \in C\left([0, T], H^{3 / 2}(\mathbb{R})\right)$ is that

$$
\lim _{\delta \rightarrow 0} \sup _{0 \leq t \leq T}\left\|\ln z_{\alpha}(\alpha, t)\right\|_{\mathrm{BMO}(\mathbb{R}), \delta}=0 .
$$

Therefore the solution obtained in Theorem 1.4 satisfies both properties (i) and (ii) (for $(a, b)=\mathbb{R})$ of Theorem 1.2, so it is analytic on $\mathbb{R}$ for each fixed $t \in(0, T)$.

Theorem 1.4 states that if only half of the data $z_{\alpha}(\alpha, 0)$ is given, there is a solution of the Birkhoff-Rott equation (1.6) for a finite time period. In general, there can be more than one solution admitting this half data $w_{0}$; this is evident in the proof of Theorem 7.8 (consequently, Theorem 1.4), in which the choice of $v_{T}$ is not unique (see Section 7).

Theorem 1.4 is a generalization of the existence result of Duchon and Robert [22] to general data. While it is reasonable to expect an existence result under some weaker regularity assumption $w_{0} \in H^{1 / 2}(\mathbb{R})$, we opt for the version presented in Theorem 1.4, with a shorter and less technical proof (see Section 7).

The following result implies that Theorem 1.4 is optimal in the sense that, in general, there is no solution of the Birkhoff-Rott equation satisfying properties (i) and (ii) as stated in Theorem 1.2 beyond the initial time $t=0$ for arbitrarily given data.

THEOREM 1.5 Assume that $z \in H^{1}\left([0, T], L_{\mathrm{loc}}^{2}(\mathbb{R})\right) \cap L^{2}\left([0, T], H_{\mathrm{loc}}^{1}(\mathbb{R})\right)$ is a solution of the Birkhoff-Rott equation (1.6) for $0 \leq t \leq T, T>0$, satisfying properties (i) and (ii) on some interval $(a, b)$ as stated in Theorem 1.2. Assume further that $w_{0}=\operatorname{Im}\left\{(1+i) \ln z_{\alpha}(\cdot, 0)\right\}$ is analytic on $(a, b)$. Then $z_{\alpha} \in C((a, b) \times$ $[0, T))$ and $\operatorname{Re}\left\{(1+i) \ln z_{\alpha}(\cdot, 0)\right\}$ is also analytic on $(a, b)$.

The reason that Theorems 1.2 through 1.5 hold is that under assumptions (i) and (ii) in Theorem 1.2, the Birkhoff-Rott equation (1.6) is of "elliptic" type on $(a, b) \times$ $(0, T)$. From the result of David, we know that assumption (i) already implies that $\ln z_{\alpha}(\cdot, t) \in \operatorname{BMO}(\mathbb{R})$. So assumption (ii) is just a further "smallness" assumption 
on section $(a, b)$. It would be interesting to see whether assumption (ii) can be removed or whether one can construct a nonsmooth solution of equation (1.6) that violates assumption (ii). At this point, it appears that assumption (ii) is a technical assumption necessary for the proof presented in this paper. Furthermore, it would be interesting to relax assumption (i) by considering vortex strength that is not necessarily bounded away from 0 and $\infty$. We refer to [50] for further discussions on open problems.

In Section 2, we give notation and some results in harmonic analysis that are needed in the proofs of Theorems 1.2 through 1.5. In Sections 3 through 6, we give proofs of Theorem 1.2 and 1.5. In particular, in Section 3 we prove that under the assumption of Theorem 1.2, the solution $z_{\alpha} \in H_{\mathrm{loc}}^{1}((a, b) \times(0, T))$ and the initial data is $H_{\text {loc }}^{1 / 2}(a, b)$ compatible (see Proposition 3.3 for the precise statement of the result and the definition of $H_{\text {loc }}^{1 / 2}$ compatibility). In Section 3, we prove that under the assumption of Theorem 1.2, the solution $z_{\alpha} \in H_{\mathrm{loc}}^{2}((a, b) \times(0, T))$ and the initial data is $H_{\mathrm{loc}}^{3 / 2}(a, b)$ compatible (Proposition 4.1). In Section 5, we prove that the solution is $C^{\infty}$ and the initial data is $C^{\infty}$ compatible (Proposition 5.1). In Section 6, we prove that the solution is analytic and analytically compatible initially and therefore complete the proof of Theorems 1.2 and 1.5. In Section 7, we prove Theorem 1.4.

The main difficulty lies in the proof of $H^{1}$ and $H^{2}$ regularity of the solution and the $H^{1 / 2}$ and $H^{3 / 2}$ compatibility of the initial data, since minimum regularity is assumed on the solution. Once we obtain the $H^{2}$ regularity of the solution and $H^{3 / 2}$ compatibility of the data, it becomes relatively easier to obtain the $C^{\infty}$ regularity and analyticity of the solution and $C^{\infty}$ and analytic compatibility of the data.

In the following, we will omit the p. v. in front of integrals. It is understood that the integrals are in principal value when necessary.

\section{Notation and Some Results from Harmonic Analysis}

Besides that introduced in the introduction, we use the following notation and abbreviations:

We write $\|b\|_{\mathrm{BMO}(\mathbb{R})}=\|b\|_{*},\|b\|_{\mathrm{BMO}(a, b), \delta_{0}}=\|b\|_{*, \delta_{0}}$, and $\|f\|_{L^{2}(\mathbb{R})}=\|f\|_{2}$. For a bounded linear operator $T: L^{2}\left(\mathbb{R}^{n}\right) \rightarrow L^{2}\left(\mathbb{R}^{n}\right),\|T\|_{2,2}$ indicates the $L^{2} \rightarrow$ $L^{2}$ operator norm of $T$.

For a set $S \subset \mathbb{R}^{n}, S^{\mathrm{C}}=\mathbb{R}^{n} \backslash S$ is the complement of $S, \chi_{S}$ is the characteristic function over the set $S$, and $\partial S$ is the boundary of the set $S$. For an interval $I \subset \mathbb{R}$ and $\lambda>0, \lambda I$ is the interval having the same center as $I$ and of length $|\lambda I|=\lambda|I|$.

For a one-variable function $f=f(x), f^{\prime}=f_{x}=\partial_{x} f=\frac{d}{d x} f, f^{\prime \prime}$, and $f^{(j)}$ indicate the first-, second-, and $j^{\text {th }}$-order derivatives of $f$. For a multivariable function $f=f(\alpha, t), f_{t}=\partial_{t} f, f_{\alpha}=\partial_{\alpha} f, f_{\alpha \alpha \alpha}=\partial_{\alpha}^{3} f$, and $f_{\alpha t}=\partial_{\alpha} \partial_{t} f$, etc., 
indicate the partial derivatives of $f$, while

$$
P(D) f(\alpha, t)=\frac{1}{\sqrt{2 \pi}} \int P(\xi) \hat{f}(\xi, t) e^{i \alpha \xi} d \xi,
$$

where $\hat{f}(\xi, t)=(1 / \sqrt{2 \pi}) \int f(\alpha, t) e^{-i \alpha \xi} d \alpha$ is the Fourier transform of $f$ in $\alpha$, always indicates the pseudodifferential operator $P(D)$ in the space variable $\alpha$. In particular,

$$
|D| f(\alpha, t)=\frac{1}{\pi} \int \frac{f(\alpha, t)-f(\beta, t)}{(\alpha-\beta)^{2}} d \beta
$$

For a one-variable function $f=f(x)$,

$$
H f(x)=\frac{1}{\pi} \int \frac{1}{x-y} f(y) d y
$$

is the Hilbert transform. For a multivariable function $f=f(\alpha, t)$,

$$
H f(\alpha, t)=\frac{1}{\pi} \int \frac{1}{\alpha-\beta} f(\beta, t) d \beta
$$

always indicates the Hilbert transform in the space variable $\alpha$. Notice that $|D|=$ $H \partial_{\alpha}$.

We write $\varphi^{h}(\alpha)=(1 /|h|) \varphi(\alpha / h)$ for any one-variable function $\varphi, h \neq 0$.

We will use the difference quotient argument to prove Theorems 1.2 and 1.5. We introduce the following notation: for a function $f=f(\alpha, t), h \neq 0$,

- $f_{h}(\alpha, t)=\frac{1}{h}(f(\alpha+h, t)-f(\alpha, t))$,

- $[f]_{h}(\alpha, t)=\frac{1}{h} \int_{\alpha}^{\alpha+h} f(\gamma, t) d \gamma$, and

- $\tilde{f}_{h}(\alpha, t)=\frac{1}{h}\left(f(\alpha, t)-[f]_{h}(\alpha, t)\right)$.

Notice that the difference quotient is always in the space variable $\alpha$. Similarly, for a one-variable function $f=f(\alpha)$,

- $f_{h}(\alpha)=\frac{1}{h}(f(\alpha+h)-f(\alpha))$,

- $[f]_{h}(\alpha)=\frac{1}{h} \int_{\alpha}^{\alpha+h} f(\gamma) d \gamma$, and

- $\tilde{f}_{h}(\alpha)=\frac{1}{h}\left(f(\alpha)-[f]_{h}(\alpha)\right)$.

Therefore $f_{h}(\alpha)=\left[f_{\alpha}\right]_{h}(\alpha)$. We will mainly work on difference quotients in $\alpha$.

We use $\mathfrak{S}\left(\mathbb{R}^{n}\right)$ to indicate the Schwartz class of functions on $\mathbb{R}^{n}$, i.e., those functions that are infinitely differentiable and decay rapidly at infinity. $C^{j, 1}\left(\mathbb{R}^{n}\right)$ consists of functions $f \in C^{j}\left(\mathbb{R}^{n}\right)$ with its $j^{\text {th }}$-order derivatives Lipschitz. For an open subset $U \subset \mathbb{R}^{n}, C_{0}^{i}(U), C_{0}^{\infty}(U)$, and $L_{0}^{\infty}(U)$ consist of functions in $C^{i}(U)$, $C^{\infty}(U)$, and $L^{\infty}(U)$, respectively, with compact support in $U . H^{s}(U)$ indicates the Sobolev space over the set $U . H_{0}^{s}(U)$ is the closure of $C_{0}^{\infty}(U)$ in $H^{s}(U)$. $L_{\mathrm{loc}}^{p}(U), H_{\mathrm{loc}}^{s}(U)$, etc., consist of functions $f$ such that $f \in L^{p}(K), H^{s}(K)$, etc., respectively, for any compact subset $K \subset U$. In this paper, we mostly work on $\mathbb{R}^{n}=\mathbb{R}, \mathbb{R}^{2}$, and $U \subset \mathbb{R}, \mathbb{R}^{2}$. In particular, we use the norm $\|f\|_{H^{s}(\mathbb{R})}=\left(\int(1+\right.$ $\left.\left.|\xi|^{2}\right)^{s}|\hat{f}(\xi)|^{2} d \xi\right)^{1 / 2}$ (here $\hat{f}$ is the Fourier transform of $f$ ), while on an interval $I \subset \mathbb{R}, I \neq \mathbb{R}$, we use $\|f\|_{H^{k}(I)}=\sum_{j=0}^{k}\left\|f^{(j)}\right\|_{L^{2}(I)}$ for nonnegative integer $k$. 
We state a few results from harmonic analysis that will be used in the proof of our theorems. Most of the material can be found in [8] and [25].

Let $\Delta=\left\{(x, y) \in \mathbb{R}^{n} \times \mathbb{R}^{n}: x=y\right\}$. A standard kernel is a continuous and differentiable function $K: \Delta^{\mathrm{c}} \rightarrow \mathbb{C}$ for which there exists a positive constant $C$ such that for all $(x, y) \in \Delta^{\mathrm{c}}$,

$$
\begin{aligned}
|K(x, y)| & \leq \frac{C}{|x-y|^{n}}, \\
\left|\nabla_{x} K(x, y)\right|+\left|\nabla_{y} K(x, y)\right| & \leq \frac{C}{|x-y|^{n+1}} .
\end{aligned}
$$

An operator $T$ taking $C_{0}^{\infty}\left(\mathbb{R}^{n}\right)$ to $L_{\text {loc }}^{1}\left(\mathbb{R}^{n}\right)$ is a Calderón-Zygmund operator (CZO) if

(1) $T$ extends to a bounded linear operator on $L^{2}\left(\mathbb{R}^{n}\right)$ to $L^{2}\left(\mathbb{R}^{n}\right)$, and

(2) there exists a standard kernel $K$ such that for every $f \in L_{0}^{\infty}\left(\mathbb{R}^{n}\right)$,

$$
T f(x)=\int K(x, y) f(y) d y \quad \text { a.e. on }\{\operatorname{supp} f\}^{\mathrm{c}} .
$$

We let $\|T\|_{c z}=\|T\|_{2,2}+C(K)$; here $C(K)$ is the smallest constant $C$ associated with the standard kernel.

A nonnegative function $\mathfrak{w}: \mathbb{R}^{n} \rightarrow \mathbb{R}$ is called an $A_{p}$ weight if there exists a constant $C>0$ such that for all cubes $Q$,

$$
m_{Q}(\mathfrak{w})\left[m_{Q}\left(\mathfrak{w}^{-\frac{1}{p-1}}\right)\right]^{p-1} \leq C .
$$

THEOREM 2.1 ([9]) If $1<p<\infty$ and $\mathfrak{w} \in A_{p}$, then every CZO $T$ is bounded on $L^{p}(\mathfrak{w} d x)$.

The following is a consequence of the " $T 1$ theorem" of Journé and David [18]. For the statement of the $T 1$ theorem in a general setting, see [18].

TheOREM 2.2 ([18]) Assume that $T: C_{0}^{\infty}\left(\mathbb{R}^{n}\right) \rightarrow L_{\mathrm{loc}}^{1}\left(\mathbb{R}^{n}\right)$ is a linear operator defined by an antisymmetric standard kernel $K: K(x, y)=-K(y, x)$ and (2.2). Then the necessary and sufficient condition for $T$ to extend to a bounded operator from $L^{2}\left(\mathbb{R}^{n}\right)$ to $L^{2}\left(\mathbb{R}^{n}\right)$ is that $T 1, T^{*} 1 \in \mathrm{BMO}$. Here $T^{*}$ is the dual operator of $T$.

Let $f: X \rightarrow Y$ be a function between two Banach spaces $X$ and $Y$ over complex numbers $\mathbb{C}$. We say that $f: X \rightarrow Y$ is holomorphic in a neighborhood of $x_{0} \in X$ if there is a $r_{0}>0$ such that $f$ is bounded and Gateaux-differentiable on $B_{r_{0}}\left(x_{0}\right)=\left\{x \in X:\left\|x-x_{0}\right\|<r_{0}\right\}$.

Let $B\left(L^{2}, L^{2}\right)$ be the space of bounded linear operators from $L^{2}\left(\mathbb{R}^{1}\right)$ to $L^{2}\left(\mathbb{R}^{1}\right)$ with norm $\|\cdot\|_{2,2}$. Let BMO be the space of complex-valued BMO functions. For $a \in \mathbf{B M O}\left(\mathbb{R}^{1}\right)$ we define the Cauchy integral operator

$$
C(a)(f)=\frac{1}{\pi} \int \frac{e^{i a(y)}}{\int_{y}^{x} e^{i a(t)} d t} f(y) d y .
$$


We know that there is a $r_{0}>0$ such that if $\|a\|_{\mathrm{BMO}(\mathbb{R})} \leq r_{0}, C(a)$ defines a bounded operator from $L^{2}\left(\mathbb{R}^{1}\right)$ to $L^{2}\left(\mathbb{R}^{1}\right)$ (see $[5,8,11,17]$ and Theorem 1.1). This can be shown as a consequence of Theorems 2.1 and 2.2. Moreover, we have the following:

THEOREM $2.3([8,12,13])$ There is a $r_{0}>0$ such that the function $C$ defined in (2.3) is holomorphic from $B_{r_{0}}(0) \subset \mathbf{B M O}\left(\mathbb{R}^{1}\right)$ to $B\left(L^{2}, L^{2}\right)$.

We have the following corollary of Theorem 2.3.

COROLlary 2.4 ([8]) Let $a \in \mathbf{B M O}\left(\mathbb{R}^{1}\right)$ with $\|a\|_{\mathrm{BMO}(\mathbb{R})}<r_{0}$; here $r_{0}$ is as in Theorem 2.3. Then $C(a)-H: L^{2}\left(\mathbb{R}^{1}\right) \rightarrow L^{2}\left(\mathbb{R}^{1}\right)$ is bounded, and there is a constant $C_{0}>0$ such that

$$
\|C(a)-H\|_{2,2} \leq C_{0}\|a\|_{\mathrm{BMO}(\mathbb{R})} .
$$

Another corollary of Theorem 2.2 is the $L^{2}$ boundedness of the Calderón commutators:

$$
C_{a}^{(k)} f(x)=\int \frac{(A(x)-A(y))^{k}}{(x-y)^{k+1}} f(y) d y .
$$

COROLlaRY 2.5 ([18]) There is a constant $C>0$ such that for all $A$, with $A^{\prime} \in$ $L^{\infty}(\mathbb{R}), f \in L^{2}(\mathbb{R})$, and positive integer $k$,

$$
\left\|C_{a}^{(k)} f\right\|_{2} \leq C^{k}\left\|A^{\prime}\right\|_{\infty}^{k}\|f\|_{2} .
$$

The following are some other useful results. Let $T$ be a CZO, $a$ a function, and $k$ a positive integer. Define the commutator

$$
M_{1} f=[a, T] f=a T(f)-T(a f) \quad \text { and } \quad M_{k+1} f=\left[a, M_{k}\right] f .
$$

THEOREM $2.6([8,14])$ Let $T$ be a $C Z O, a \in \mathrm{BMO}\left(\mathbb{R}^{1}\right)$, and $k$ a positive integer. Then there exist constants $C_{k}>0$ depending on $\|T\|_{c z}$ such that the operator $M_{k}$ is bounded from $L^{2}\left(\mathbb{R}^{1}\right)$ to $L^{2}\left(\mathbb{R}^{1}\right)$ and

$$
\left\|M_{k}\right\|_{2,2} \leq C_{k}\|a\|_{\mathrm{BMO}(\mathbb{R})}^{k} .
$$

PROPOSITION 2.7 ([8])

(i) Assume that $b \in \mathbf{B M O}(\mathbb{R})$, with $\operatorname{Re} b \in L^{\infty}(\mathbb{R}),\|\operatorname{Re} b\|_{L^{\infty}} \leq N$. There exist constants $r_{1}, C_{1}>0$, depending on $N$, such that if $\|b\|_{\mathrm{BMO}(\mathbb{R})} \leq r_{1}$, then $e^{b} \in \mathbf{B M O}(\mathbb{R})$, and

$$
\left\|e^{b}\right\|_{\mathrm{BMO}(\mathbb{R})} \leq C_{1}\|b\|_{\mathrm{BMO}(\mathbb{R})} .
$$

(ii) Assume that $b \in \mathbf{B M O}(\mathbb{R})$, with $\ln |b| \in L^{\infty}(\mathbb{R})$ and $\|\ln |b|\|_{L^{\infty}} \leq N$. There exist constants $r_{1}, C_{1}>0$, depending on $N$, such that if $\|b\|_{\mathrm{BMO}(\mathbb{R})} \leq r_{1}$, then there exist a determination of $\ln b$, with $\ln b \in \mathrm{BMO}(\mathbb{R})$ and

$$
\|\ln b\|_{\mathrm{BMO}(\mathbb{R})} \leq C_{1}\|b\|_{\mathrm{BMO}(\mathbb{R})} .
$$


Now assume that $b \in \mathrm{BMO}([c, d])$; we define the reflective periodic extension $a$ of $b$ by

$$
a(x)= \begin{cases}b(x), & x \in[c, d], \\ b(2 c-x), & x \in[2 c-d, c],\end{cases}
$$

$a$ of period $2(d-c)$. Then the following holds:

PROpOSITION 2.8 ([8]) Let $b \in \mathrm{BMO}([c, d])$ and $a$ be the reflective periodic extension of $b$ to $\mathbb{R}$. Then $a \in \mathrm{BMO}(\mathbb{R})$ and

$$
\|a\|_{\mathrm{BMO}(\mathbb{R})} \leq 8\|b\|_{\mathrm{BMO}[c, d]} .
$$

In the following, $c_{1}, c_{2}, c^{\prime}, c^{\prime \prime}$, etc., are used to indicate constants. Constants appearing in different contexts may bear the same notation, although they are not necessarily the same.

More notation will be introduced along the way.

\section{$3 H^{1}$ Regularity of $z_{\alpha}$ and $H^{1 / 2}$ Compatibility of the Initial Data}

Assume that $z \in H^{1}\left([0, T], L_{\text {loc }}^{2}(\mathbb{R})\right) \cap L^{2}\left([0, T], H_{\mathrm{loc}}^{1}(\mathbb{R})\right)$ is a solution of the Birkhoff-Rott equation (1.6) for $0 \leq t \leq T$, satisfying assumption (i) of Theorem 1.2. We want to show that there exists a constant $c(m, M)>0$ such that if on some fixed interval $(a, b)$ there exists a $\delta_{0}>0$ such that for some determination of $\ln z_{\alpha}$ we have

$$
\sup _{[0, T]}\left\|\ln z_{\alpha}(\cdot, t)\right\|_{\operatorname{BMO}(a, b), \delta_{0}} \leq c(m, M),
$$

then $\ln z_{\alpha} \in H^{1}(K)$ for any compact subset $K$ of $(a, b) \times(0, T)$. We also want to show that if, in addition, $\operatorname{Im}\left((1+i) \ln z_{\alpha}(\cdot, 0)\right) \in H_{\text {loc }}^{1 / 2}(a, b)$, then we have $\operatorname{Re}\left((1+i) \ln z_{\alpha}(\cdot, 0)\right) \in H_{\mathrm{loc}}^{1 / 2}(a, b)$ and $\ln z_{\alpha} \in H^{1}(K)$ for any compact subset $K$ of $(a, b) \times[0, T)$.

\subsection{A Heuristic Argument}

We first give a heuristic argument, since it better illustrates the main ideas. We assume $(a, b)$ is of finite length, $\ln z_{\alpha} \in L^{2}((a, b) \times[0, T])$, and all the terms that appear are finite, so that subtraction of terms makes sense. For any compact subset $K$ of $(a, b) \times(0, T)$, we will derive an estimate that bounds $\left\|\ln z_{\alpha}\right\|_{H^{1}(K)}$ by $\left\|\ln z_{\alpha}\right\|_{L^{2}((a, b) \times[0, T])}$. In the process, we will find the constant $c(m, M)$. We begin with $c(m, M) \leq \min \left\{r_{0} / 8, r_{1} / 8\right\}$; here $r_{0}$ and $r_{1}$ are as in Corollary 2.4 and Proposition 2.7.

Let $Q$ be an interval satisfying $2 Q \subset(a, b)$ and $|2 Q| \leq \delta_{0}$. Take $\eta, \rho \in C_{0}^{\infty}(\mathbb{R})$ such that

$$
\begin{array}{ll}
\eta(\alpha)=1 \text { on } Q, & \operatorname{supp} \eta \subset \frac{5}{4} Q, \\
\rho(\alpha)=1 \text { on } \frac{3}{2} Q, & \text { supp } \rho \subset 2 Q,
\end{array}
$$


and $0 \leq \eta(\alpha), \rho(\alpha) \leq 1$, and $\left|\eta^{\prime}(\alpha)\right|+\left|\rho^{\prime}(\alpha)\right| \leq C /|Q|$ for some constant $C>0$. Taking the derivative with respect to $\alpha$ on both sides of (1.6) and using integration by parts, we obtain that for $\alpha \in \frac{5}{4} Q$

$$
\begin{aligned}
\bar{z}_{t \alpha}(\alpha, t)= & \frac{1}{2 \pi i} \int \frac{z_{\alpha}(\alpha, t)}{z(\alpha, t)-z(\beta, t)} \rho(\beta) \partial_{\beta} \frac{1}{z_{\beta}} d \beta \\
& +\frac{1}{2 \pi i} \int \frac{z_{\alpha}(\alpha, t)}{(z(\alpha, t)-z(\beta, t)) z_{\beta}} \rho^{\prime}(\beta) d \beta \\
& -\frac{1}{2 \pi i} \int \frac{z_{\alpha}(\alpha, t)(1-\rho(\beta))}{(z(\alpha, t)-z(\beta, t))^{2}} d \beta
\end{aligned}
$$

Let

$$
\begin{aligned}
F_{1}(\alpha, t)= & \frac{1}{2 \pi i} \int \frac{z_{\alpha}}{(z(\alpha, t)-z(\beta, t)) z_{\beta}} \rho^{\prime}(\beta) d \beta \\
& -\frac{1}{2 \pi i} \int \frac{z_{\alpha}(1-\rho(\beta))}{(z(\alpha, t)-z(\beta, t))^{2}} d \beta .
\end{aligned}
$$

For $\alpha \in \frac{5}{4} Q$ and $t \in[0, T]$, we have from assumption (i) that

$$
\left|F_{1}(\alpha, t)\right| \leq \frac{c_{1}}{|Q|}
$$

for some constant $c_{1}$. Now

$$
\begin{aligned}
& \int \frac{z_{\alpha}(\alpha, t)}{z(\alpha, t)-z(\beta, t)} \rho(\beta) \partial_{\beta} \frac{1}{z_{\beta}} d \beta \\
& =\int \frac{1}{\alpha-\beta} \rho(\beta) \partial_{\beta} \frac{1}{z_{\beta}} d \beta+\int\left(\frac{z_{\alpha}(\alpha, t)}{z(\alpha, t)-z(\beta, t)}-\frac{1}{\alpha-\beta}\right) \rho(\beta) \partial_{\beta} \frac{1}{z_{\beta}} d \beta \\
& =-\frac{1}{z_{\alpha}} \int \frac{1}{\alpha-\beta} \rho(\beta) \partial_{\beta} \ln z_{\beta} d \beta+\pi\left[\frac{1}{z_{\alpha}}, H\right]\left(\rho \partial_{\alpha} \ln z_{\alpha}\right) \\
& \quad-\int\left(\frac{z_{\alpha}(\alpha, t)}{z(\alpha, t)-z(\beta, t)}-\frac{1}{\alpha-\beta}\right) \rho(\beta) \frac{1}{z_{\beta}} \partial_{\beta} \ln z_{\beta} d \beta
\end{aligned}
$$

Let

$$
\begin{aligned}
F_{2}(\alpha, t)= & \pi\left[\frac{1}{z_{\alpha}}, H\right]\left(\rho \partial_{\alpha} \ln z_{\alpha}\right) \\
& -\int\left(\frac{z_{\alpha}(\alpha, t)}{z(\alpha, t)-z(\beta, t)}-\frac{1}{\alpha-\beta}\right) \rho(\beta) \frac{1}{z_{\beta}} \partial_{\beta} \ln z_{\beta} d \beta .
\end{aligned}
$$

For fixed $t \in[0, T]$, using a reflective periodic extension of $\ln z_{\alpha}(\alpha, t)$ from $\alpha \in$ $2 Q$ to $\mathbb{R}$ when necessary, applying Theorem 2.6 to the first term and Corollary 2.4 to the second term, and furthermore, using Propositions 2.7 and 2.8, we get

$$
\int \eta^{2}(\alpha)\left|F_{2}(\alpha, t)\right|^{2} d \alpha \leq c_{2}\left\|\ln z_{\alpha}\right\|_{*, \delta_{0}}^{2} \int\left|\rho(\alpha) \partial_{\alpha} \ln z_{\alpha}(\alpha, t)\right|^{2} d \alpha
$$


for some constant $c_{2}$ independent of $t$. Therefore

$$
\partial_{t}\left(\ln \bar{z}_{\alpha}\right)=-\frac{1}{2 \pi i\left|z_{\alpha}\right|^{2}} \int \frac{1}{\alpha-\beta} \rho(\beta) \partial_{\beta} \ln z_{\beta} d \beta+\frac{1}{\bar{z}_{\alpha}}\left(\frac{1}{2 \pi i} F_{2}+F_{1}\right) .
$$

Multiplying $\eta(\alpha)$ on both sides of equation (3.6) gives

$$
\begin{aligned}
\eta(\alpha) \partial_{t}\left(\ln \bar{z}_{\alpha}\right)= & -\frac{1}{2 \pi i\left|z_{\alpha}\right|^{2}} \int \frac{\eta(\beta)}{\alpha-\beta} \partial_{\beta} \ln z_{\beta} d \beta \\
& -\frac{1}{2 \pi i\left|z_{\alpha}\right|^{2}} \int \frac{\eta(\alpha)-\eta(\beta)}{\alpha-\beta} \rho(\beta) \partial_{\beta} \ln z_{\beta} d \beta \\
& +\frac{\eta(\alpha)}{\bar{z}_{\alpha}}\left(\frac{1}{2 \pi i} F_{2}+F_{1}\right) .
\end{aligned}
$$

Now by writing

$$
\int \frac{\eta(\beta)}{\alpha-\beta} \partial_{\beta} \ln z_{\beta} d \beta=\int \frac{1}{\alpha-\beta} \partial_{\beta}\left(\eta(\beta) \ln z_{\beta}\right) d \beta-\int \frac{\eta^{\prime}(\beta)}{\alpha-\beta} \ln z_{\beta} d \beta,
$$

we get from (3.7) that

$$
\eta(\alpha) \partial_{t}\left(\ln \bar{z}_{\alpha}\right)=-\frac{1}{2 \pi i\left|z_{\alpha}\right|^{2}} \int \frac{1}{\alpha-\beta} \partial_{\beta}\left(\eta(\beta) \ln z_{\beta}\right) d \beta+F(\alpha, t),
$$

where

$$
\begin{aligned}
F(\alpha, t)= & \frac{1}{2 \pi i\left|z_{\alpha}\right|^{2}}\left\{\int \frac{\eta^{\prime}(\beta)}{\alpha-\beta} \ln z_{\beta} d \beta-\int \frac{\eta(\alpha)-\eta(\beta)}{\alpha-\beta} \rho(\beta) \partial_{\beta} \ln z_{\beta} d \beta\right\} \\
& +\frac{\eta(\alpha)}{\bar{z}_{\alpha}}\left(\frac{1}{2 \pi i} F_{2}+F_{1}\right) .
\end{aligned}
$$

From (3.4), (3.5), Corollary 2.5, and Theorem 2.6, there exist constants $c_{1}, c_{2}$, and $c_{3}$, independent of $t \in[0, T]$, such that

$$
\begin{aligned}
\int|F(\alpha, t)|^{2} d \alpha \leq & c_{1}\left\|\ln z_{\alpha}\right\|_{*, \delta_{0}}^{2} \int\left|\rho(\alpha) \partial_{\alpha} \ln z_{\alpha}(\alpha, t)\right|^{2} d \alpha \\
& +\frac{c_{2}}{|Q|^{2}} \int_{2 Q}\left|\ln z_{\alpha}\right|^{2} d \alpha+\frac{c_{3}}{|Q|} .
\end{aligned}
$$

Let $V=(1+i) \ln z_{\alpha}$. We get from (3.8) that

$$
\eta \overline{V_{t}}=\frac{1}{2\left|z_{\alpha}\right|^{2}}|D|(\eta V)+(1-i) F(\alpha, t)
$$

We now estimate

$$
\int\left|z_{\alpha}(\alpha, t)\right|^{2}\left|\eta(\alpha) \overline{V_{t}}(\alpha, t)-\frac{1}{2\left|z_{\alpha}\right|^{2}}\right| D|(\eta V)(\alpha, t)|^{2} d \alpha
$$

We have the following identity: 
Proposition 3.1

$$
\begin{aligned}
\int\left|z_{\alpha}\right|^{2}\left|\eta \overline{V_{t}}-\frac{1}{2\left|z_{\alpha}\right|^{2}}\right| D|(\eta V)|^{2} d \alpha \\
=\int\left(\left|z_{\alpha}\right|^{2}\left|\eta(\alpha) V_{t}\right|^{2}+\frac{1}{4\left|z_{\alpha}\right|^{2}}|| D|(\eta V)|^{2}\right) d \alpha \\
\quad-\frac{1}{2} \operatorname{Re} \frac{d}{d t} \int \eta(\alpha) V(\alpha, t)|D|(\eta V)(\alpha, t) d \alpha .
\end{aligned}
$$

Proof: Notice that $|D|: L^{2}(\mathbb{R}) \rightarrow L^{2}(\mathbb{R})$ is a self-adjoint operator. We have

$$
\begin{aligned}
\int\left|z_{\alpha}\right|^{2}\left|\eta \overline{V_{t}}-\frac{1}{2\left|z_{\alpha}\right|^{2}}\right| D|(\eta V)|^{2} d \alpha \\
=\int\left(\left|z_{\alpha}\right|^{2}\left|\eta V_{t}\right|^{2}+\frac{1}{4\left|z_{\alpha}\right|^{2}}|| D|(\eta V)|^{2}\right) d \alpha-\operatorname{Re} \int \eta V_{t}|D|(\eta V) d \alpha \\
=\int\left(\left|z_{\alpha}\right|^{2}\left|\eta(\alpha) V_{t}\right|^{2}+\frac{1}{4\left|z_{\alpha}\right|^{2}}|| D|(\eta V)|^{2}\right) d \alpha \\
\quad-\frac{1}{2} \operatorname{Re} \frac{d}{d t} \int \eta(\alpha) V(\alpha, t)|D|(\eta V)(\alpha, t) d \alpha .
\end{aligned}
$$

Assumption (i) of Theorem 1.2 implies that $m \leq\left|z_{\alpha}\right| \leq M$; therefore from (3.10) and (3.11) we have

$$
\begin{aligned}
m^{2} \int\left|\eta V_{t}\right|^{2} d \alpha & +\frac{1}{4 M^{2}} \int|| D|(\eta V)|^{2} d \alpha \\
& \leq \frac{1}{2} \operatorname{Re} \frac{d}{d t} \int \eta V|D|(\eta V) d \alpha+2 M^{2} \int|F(\alpha, t)|^{2} d \alpha
\end{aligned}
$$

Taking $\delta(t)=t^{2}(T-t)^{2}$ for $t \in[0, T]$ and $\delta(t)=0$ for $t \notin[0, T]$, integrating with respect to $t$, and using integration by parts, we get

$$
\begin{aligned}
& m^{2} \iint \delta(t)\left|\eta V_{t}\right|^{2} d \alpha d t+\frac{1}{4 M^{2}} \iint \delta(t)|| D|(\eta V)|^{2} d \alpha d t \\
& \leq-\frac{1}{2} \operatorname{Re} \iint \delta^{\prime}(t) \eta V|D|(\eta V) d \alpha d t+2 M^{2} \iint \delta(t)|F(\alpha, t)|^{2} d \alpha d t \\
& \leq 2 M^{2} T^{2} \int_{0}^{T} \int|\eta V|^{2} d \alpha d t+\frac{1}{8 M^{2}} \iint \delta(t)|| D|(\eta V)|^{2} d \alpha d t \\
& \quad+2 M^{2} \iint \delta(t)|F(\alpha, t)|^{2} d \alpha d t .
\end{aligned}
$$

Here we used the fact that $\delta^{\prime}(t)^{2} \leq 4 T^{2} \delta(t)$ for $t \in[0, T]$ and the Hölder inequality. Therefore 


$$
\begin{aligned}
& m^{2} \iint \delta(t)\left|\eta V_{t}\right|^{2} d \alpha d t+\frac{1}{8 M^{2}} \iint \delta(t)|| D|(\eta V)|^{2} d \alpha d t \\
& \leq 2 M^{2} T^{2} \int_{0}^{T} \int|\eta V|^{2} d \alpha d t+2 M^{2} \iint \delta(t)|F(\alpha, t)|^{2} d \alpha d t
\end{aligned}
$$

Using (3.9) and that

$$
\int|| D|(\eta V)|^{2} d \alpha=\int\left|\partial_{\alpha}(\eta V)\right|^{2} d \alpha \geq \frac{1}{2} \int\left|\eta V_{\alpha}\right|^{2} d \alpha-\int\left|\eta^{\prime}(\alpha) V\right|^{2} d \alpha,
$$

we arrive at

$$
\begin{gathered}
m^{2} \iint \delta(t)\left|\eta V_{t}\right|^{2} d \alpha d t+\frac{1}{16 M^{2}} \iint \delta(t)\left|\eta V_{\alpha}\right|^{2} d \alpha d t \\
\leq 2 M^{2} c_{1} \sup _{[0, T]}\left\|\ln z_{\alpha}\right\|_{*, \delta_{0}}^{2} \iint \delta(t)\left|\rho(\alpha) V_{\alpha}\right|^{2} d \alpha d t \\
\quad+\left(c_{2}+\frac{c_{3}}{|Q|^{2}}\right) \int_{0}^{T} \int_{2 Q}|V|^{2} d \alpha d t+\frac{c_{4}}{|Q|}
\end{gathered}
$$

for some constants $c_{i}, i=1, \ldots, 4$. Now if we take the number $c(m, M)$ in (3.1) by

$$
c(m, M)=\min \left\{\frac{r_{0}}{8}, \frac{r_{1}}{8}, \frac{1}{20 M^{2} \sqrt{c_{1}}}\right\}
$$

where $r_{0}$ is as in Corollary 2.4 and $r_{1}$ is as in Proposition 2.7, we get

$$
\begin{aligned}
m^{2} \int \delta(t) \int_{Q}\left|V_{t}\right|^{2} d \alpha d t+\frac{1}{16 M^{2}} \int \delta(t) \int_{Q}\left|V_{\alpha}\right|^{2} d \alpha d t \\
\leq \frac{1}{200 M^{2}} \int \delta(t) \int_{2 Q}\left|V_{\alpha}\right|^{2} d \alpha d t \\
\quad+\left(c_{2}+\frac{c_{3}}{|Q|^{2}}\right) \int_{0}^{T} \int_{2 Q}|V|^{2} d \alpha d t+\frac{c_{4}}{|Q|} .
\end{aligned}
$$

We prove the following proposition:

Proposition 3.2 Let $I \subset R$ be a finite-length interval. Assume that the function $V \in H^{1}(I \times[0, T])$ satisfies (3.13) for all subintervals $2 Q \subset I$. Then there are constants $C_{1}$ and $C_{2}$ such that

$$
\begin{aligned}
& m^{2} \int \delta(t) \int_{I} \operatorname{dist}^{2}(\alpha, \partial I)\left|V_{t}\right|^{2} d \alpha d t \\
& +\frac{1}{32 M^{2}} \int \delta(t) \int_{I} \operatorname{dist}^{2}(\alpha, \partial I)\left|V_{\alpha}\right|^{2} d \alpha d t \\
& \quad \leq C_{1} \int_{0}^{T} \int_{I}|V|^{2} d \alpha d t+C_{2} .
\end{aligned}
$$


Here $\operatorname{dist}(\alpha, \partial I)$ is the distance from point $\alpha$ to the boundary $\partial I$ of the interval $I$.

ProOF: Let $I=[p, q]$. We divide the interval $I$ into a disjoint union of subintervals $I_{1, k}$ and $I_{2, k}, k=1,2, \ldots$, such that

$$
\begin{aligned}
& \left|I_{1, k}\right|=\operatorname{dist}\left(p, I_{1, k}\right)=\frac{1}{2^{k+1}}|I|, \quad \text { for } k=1,2, \ldots \\
& \left|I_{2, k}\right|=\operatorname{dist}\left(q, I_{2, k}\right)=\frac{1}{2^{k+1}}|I|,
\end{aligned}
$$

We apply (3.13) to $Q=I_{i, k}, i=1,2, k=1,2, \ldots$ Multiply $|Q|^{2}=\left|I_{i, k}\right|^{2}$ on both sides of (3.13) and then sum up with respect to $i=1,2, k=1,2, \ldots$ We get

$$
\begin{aligned}
m^{2} \sum_{i, k} \int \delta(t) \int_{I_{i, k}}\left|I_{i, k}\right|^{2}\left|V_{t}\right|^{2} d \alpha d t+\frac{1}{16 M^{2}} \sum_{i, k} \int \delta(t) \int_{I_{i, k}}\left|I_{i, k}\right|^{2}\left|V_{\alpha}\right|^{2} d \alpha d t \\
\leq \frac{1}{200 M^{2}} \sum_{i, k} \int \delta(t) \int_{2 I_{i, k}}\left|I_{i, k}\right|^{2}\left|V_{\alpha}\right|^{2} d \alpha d t \\
\quad+\sum_{i, k}\left(\left(c_{2}\left|I_{i, k}\right|^{2}+c_{3}\right) \int_{0}^{T} \int_{2 I_{i, k}}|V|^{2} d \alpha d t+c_{4}\left|I_{i, k}\right|\right) .
\end{aligned}
$$

Notice that

$$
\begin{aligned}
\sum_{i, k}\left|I_{i, k}\right|^{2} \chi_{2 I_{i, k}}(\alpha) & \leq 6 \sum_{i, k}\left|I_{i, k}\right|^{2} \chi_{I_{i, k}}(\alpha), \\
\sum_{i, k} \chi_{2 I_{i, k}}(\alpha) & \leq 3 \sum_{i, k} \chi_{I_{i, k}}(\alpha)=3 \chi_{I}(\alpha),
\end{aligned}
$$

for all $\alpha \in \mathbb{R}$; therefore

$$
\begin{aligned}
m^{2} \int \delta(t) \int \sum_{i, k}\left|I_{i, k}\right|^{2} \chi_{I_{i, k}}\left|V_{t}\right|^{2} d \alpha d t \\
+\frac{1}{16 M^{2}} \int \delta(t) \int \sum_{i, k}\left|I_{i, k}\right|^{2} \chi_{I_{i, k}}\left|V_{\alpha}\right|^{2} d \alpha d t \\
\quad \leq \frac{6}{200 M^{2}} \int \delta(t) \int \sum_{i, k}\left|I_{i, k}\right|^{2} \chi_{I_{i, k}}\left|V_{\alpha}\right|^{2} d \alpha d t \\
\quad+3\left(c_{2}|I|^{2}+c_{3}\right) \int_{0}^{T} \int_{I}|V|^{2} d \alpha d t+c_{4}|I|,
\end{aligned}
$$


and

$$
\begin{aligned}
& m^{2} \int \delta(t) \int \sum_{i, k}\left|I_{i, k}\right|^{2} \chi_{I_{i, k}}\left|V_{t}\right|^{2} d \alpha d t \\
& +\frac{1}{32 M^{2}} \int \delta(t) \int \sum_{i, k}\left|I_{i, k}\right|^{2} \chi_{I_{i, k}}\left|V_{\alpha}\right|^{2} d \alpha d t \\
& \quad \leq 3\left(c_{2}|I|^{2}+c_{3}\right) \int_{0}^{T} \int_{I}|V|^{2} d \alpha d t+c_{4}|I| .
\end{aligned}
$$

From

$$
\frac{1}{4} \operatorname{dist}^{2}(\alpha, \partial I) \leq \sum_{i, k}\left|I_{i, k}\right|^{2} \chi_{I_{i, k}}(\alpha) \quad \text { for all } \alpha \in I
$$

we get (3.14).

If we apply Proposition 3.2 to $V=(1+i) \ln z_{\alpha}$ (assume $V \in H^{1}$ ) on any interval $I \subset(a, b)$ with $|I| \leq \delta_{0}$, we get a bound on

$$
\left\|\partial_{t}\left(\ln z_{\alpha}\right)\right\|_{L^{2}\left(\frac{1}{2} I \times\left(T_{1}, T_{2}\right)\right)}+\left\|\partial_{\alpha}\left(\ln z_{\alpha}\right)\right\|_{L^{2}\left(\frac{1}{2} I \times\left(T_{1}, T_{2}\right)\right)}
$$

by $\left\|\ln z_{\alpha}\right\|_{L^{2}(I \times(0, T))}$ for any interval $\left[T_{1}, T_{2}\right] \subset(0, T)$. This suggests that we can bound the $H^{1}(K)$ norm of $\ln z_{\alpha}$ by its $L^{2}((a, b) \times[0, T])$ norm for any compact subset $K$ of $(a, b) \times(0, T)$.

Let $V(\cdot, 0)=v+i w$; here $v$ and $w$ are the real and imaginary parts of $V(\cdot, 0)$, respectively. If we assume, moreover, $w \in H^{1 / 2}(a, b)$, we can modify the above argument to get a bound on $\|v\|_{H^{1 / 2}(K)}$ for any compact subset $K$ of $(a, b)$. Taking $\zeta(t)=(T-t)^{2}$ for $t \in[0, T]$ and $\zeta(t)=0$ for $t \notin[0, T]$, we get from (3.12) that

$$
\begin{aligned}
& m^{2} \iint \zeta(t)\left|\eta V_{t}\right|^{2} d \alpha d t+\frac{1}{4 M^{2}} \iint \zeta(t)|| D|(\eta V)|^{2} d \alpha d t \\
& \quad \leq-\frac{1}{2} \operatorname{Re} \iint \zeta^{\prime}(t) \eta V|D|(\eta V) d \alpha d t \\
& \quad-\frac{1}{2} \operatorname{Re} \zeta(0) \int \eta V(\alpha, 0)|D|(\eta V)(\alpha, 0) d \alpha+2 M^{2} \iint \zeta(t)|F(\alpha, t)|^{2} d \alpha d t .
\end{aligned}
$$

Notice that

$$
\operatorname{Re}(\eta V(\cdot, 0)|D|(\eta V)(\cdot, 0))=\eta v|D|(\eta v)-\eta w|D|(\eta w)
$$

and

$$
\left|\zeta^{\prime}(t)\right|^{2} \leq 4 \zeta(t), \quad \zeta(0)=T^{2}
$$


Again using the Hölder inequality, we get

$$
\begin{aligned}
& m^{2} \iint \zeta(t)\left|\eta V_{t}\right|^{2} d \alpha d t+\frac{1}{4 M^{2}} \iint \zeta(t)|| D|(\eta V)|^{2} d \alpha d t \\
& \quad+\frac{1}{2} T^{2} \int \eta v|D|(\eta v) d \alpha \\
& \quad \leq 2 M^{2} \int_{0}^{T} \int|\eta V|^{2} d \alpha d t+\frac{1}{8 M^{2}} \iint \zeta(t)|| D|(\eta V)|^{2} d \alpha d t \\
& \quad+\frac{1}{2} T^{2} \int \eta w|D|(\eta w) d \alpha+2 M^{2} \iint \zeta(t)|F(\alpha, t)|^{2} d \alpha d t
\end{aligned}
$$

Proceeding as above and taking the same constant $c(m, M)$, we arrive at

$$
\begin{aligned}
m^{2} \int \zeta(t) \int_{Q}\left|V_{t}\right|^{2} d \alpha d t+\frac{1}{16 M^{2}} \int \zeta(t) \int_{Q}\left|V_{\alpha}\right|^{2} d \alpha d t \\
+\frac{1}{2} T^{2} \int_{Q} \eta v|D|(\eta v) d \alpha \\
\leq \frac{1}{200 M^{2}} \int \zeta(t) \int_{2 Q}\left|V_{\alpha}\right|^{2} d \alpha d t \\
+\left(c_{2}+\frac{c_{3}}{|Q|^{2}}\right) \int_{0}^{T} \int_{2 Q}|V|^{2} d \alpha d t \\
+\frac{c_{4}}{|Q|}+\frac{1}{2} T^{2} \int \eta w|D|(\eta w) d \alpha
\end{aligned}
$$

Relation (3.15) holds for all intervals $Q$, with $2 Q \subset(a, b)$ and $|2 Q| \leq \delta_{0}$.

Now for any interval $I \subset(a, b),|I| \leq \delta_{0}$, we use the same argument as in Proposition 3.2. Let $\eta_{i, k}$ be the function $\eta$ corresponding to the interval $I_{i, k}$ such that $\left|I_{i, k}\right|\left\|\eta_{i, k}^{\prime}\right\|_{\infty} \leq C$ for a constant $C$ independent of $i$ and $k$. Notice that for any function $f \in C^{\infty}(\mathbb{R})$,

$$
\begin{aligned}
& \sum_{i, k}\left|I_{i, k}\right|^{2} \int \eta_{i, k} f|D|\left(\eta_{i, k} f\right) d \alpha \\
& \quad=\frac{1}{2 \pi} \sum_{i, k}\left|I_{i, k}\right|^{2} \iint \frac{\left(\eta_{i, k}(\alpha) f(\alpha)-\eta_{i, k}(\beta) f(\beta)\right)^{2}}{(\alpha-\beta)^{2}} d \alpha d \beta \\
& \quad=\frac{1}{2 \pi} \iint \frac{\sum_{i, k}\left|I_{i, k}\right|^{2}\left\{\eta_{i, k}^{2}(\alpha) f^{2}(\alpha)+\eta_{i, k}^{2}(\beta) f^{2}(\beta)-2 \eta_{i, k}(\alpha) \eta_{i, k}(\beta) f(\alpha) f(\beta)\right\}}{(\alpha-\beta)^{2}} d \alpha d \beta .
\end{aligned}
$$


Let $\theta(\alpha) \geq 0$,

$$
\theta^{2}(\alpha)=\sum_{i, k}\left|I_{i, k}\right|^{2} \eta_{i, k}^{2}(\alpha)
$$

We have $\theta \in C^{\infty}(a, b) \cap C^{0,1}(\mathbb{R}), \operatorname{supp} \theta \subset I$, and

$$
\begin{aligned}
\frac{1}{4} \operatorname{dist}^{2}(\alpha, \partial I) \leq \sum_{i, k}\left|I_{i, k}\right|^{2} \chi_{I_{i, k}} \leq \theta^{2}(\alpha) & \leq \sum_{i, k}\left|I_{i, k}\right|^{2} \chi_{2 I_{i, k}} \\
& \leq 6 \operatorname{dist}^{2}(\alpha, \partial I)
\end{aligned}
$$

for $\alpha \in I$. Therefore

$$
\begin{aligned}
& \sum_{i, k}\left|I_{i, k}\right|^{2} \int \eta_{i, k} f|D|\left(\eta_{i, k} f\right) d \alpha \\
& =\int \theta f|D|(\theta f) d \alpha \\
& \quad+\frac{1}{\pi} \iint \frac{\theta(\alpha) \theta(\beta)-\sum_{i, k}\left|I_{i, k}\right|^{2} \eta_{i, k}(\alpha) \eta_{i, k}(\beta)}{(\alpha-\beta)^{2}} f(\alpha) f(\beta) \chi_{I}(\alpha) \chi_{I}(\beta) d \alpha d \beta .
\end{aligned}
$$

Since $2 \sum_{i, k}\left|I_{i, k}\right|^{2} \eta_{i, k}(\alpha) \eta_{i, k}(\beta) \leq 2 \theta(\alpha) \theta(\beta) \leq \theta^{2}(\alpha)+\theta^{2}(\beta)$,

$$
\begin{aligned}
& \left|\iint \frac{2 \theta(\alpha) \theta(\beta)-2 \sum_{i, k}\left|I_{i, k}\right|^{2} \eta_{i, k}(\alpha) \eta_{i, k}(\beta)}{(\alpha-\beta)^{2}} f(\alpha) f(\beta) \chi_{I}(\alpha) \chi_{I}(\beta) d \alpha d \beta\right| \\
& \quad \leq \iint \frac{2 \theta(\alpha) \theta(\beta)-2 \sum_{i, k}\left|I_{i, k}\right|^{2} \eta_{i, k}(\alpha) \eta_{i, k}(\beta)}{(\alpha-\beta)^{2}}|f(\alpha) f(\beta)| \chi_{I}(\alpha) \chi_{I}(\beta) d \alpha d \beta \\
& \quad \leq \iint \frac{\sum_{i, k}\left|I_{i, k}\right|^{2}\left(\eta_{i, k}(\alpha)-\eta_{i, k}(\beta)\right)^{2}}{(\alpha-\beta)^{2}}|f(\alpha) f(\beta)| \chi_{I}(\alpha) \chi_{I}(\beta) d \alpha d \beta
\end{aligned}
$$

From the assumption $\left|I_{i, k}\right|\left\|\eta_{i, k}^{\prime}\right\|_{\infty} \leq C$ and Corollary 2.5, there is a constant $C_{1}$ such that

$$
\begin{aligned}
& \left|\iint \frac{2 \theta(\alpha) \theta(\beta)-2 \sum_{i, k}\left|I_{i, k}\right|^{2} \eta_{i, k}(\alpha) \eta_{i, k}(\beta)}{(\alpha-\beta)^{2}} f(\alpha) f(\beta) \chi_{I}(\alpha) \chi_{I}(\beta) d \alpha d \beta\right| \\
& \quad \leq 2 \sum_{i, k}\left|I_{i, k}\right|^{2} \iint \frac{\eta_{i, k}(\alpha)\left(\eta_{i, k}(\alpha)-\eta_{i, k}(\beta)\right)}{(\alpha-\beta)^{2}}|f(\alpha) f(\beta)| \chi_{I}(\alpha) \chi_{I}(\beta) d \alpha d \beta \\
& \quad \leq C_{1} \sum_{i, k}\left|I_{i, k}\right| \int_{I} f^{2}(\alpha) d \alpha \leq C_{1}|I| \int_{I} f^{2}(\alpha) d \alpha .
\end{aligned}
$$


Applying the above calculation to $f=v$ and $f=w$, and using (3.15) and the same argument as in Proposition 3.2, we arrive at

$$
\begin{aligned}
& m^{2} \int \zeta(t) \int_{I} \operatorname{dist}^{2}(\alpha, \partial I)\left|V_{t}\right|^{2} d \alpha d t \\
& +\frac{1}{32 M^{2}} \int_{I} \zeta(t) \int_{I} \operatorname{dist}^{2}(\alpha, \partial I)\left|V_{\alpha}\right|^{2} d \alpha d t+\frac{1}{2} T^{2} \int \theta v|D|(\theta v) d \alpha \\
& \quad \leq C_{1} \int_{0}^{T} \int_{I}|V|^{2} d \alpha d t+\frac{1}{2} T^{2} \int \theta w|D|(\theta w) d \alpha \\
& \quad+C_{2}|I| \int_{I}|V(\alpha, 0)|^{2} d \alpha+C_{3}
\end{aligned}
$$

for some constants $C_{1}, C_{2}$, and $C_{3}$. This gives a bound on the $H^{1 / 2}\left(K_{1}\right)$ norm of $\operatorname{Re} V(\cdot, 0)$ on any compact subset $K_{1}$ of $(a, b)$. Furthermore, we have a bound on the $H^{1}(K)$ norm of $V=(1+i) \ln z_{\alpha}$ on any compact subset $K$ of $(a, b) \times[0, T)$. Notice that this compact set $K$ can contain the initial time $t=0$.

\subsection{A Strict Argument}

We prove the following result:

Proposition 3.3 Assume that $z \in H^{1}\left([0, T], L_{\mathrm{loc}}^{2}(\mathbb{R})\right) \cap L^{2}\left([0, T], H_{\mathrm{loc}}^{1}(\mathbb{R})\right)$ is a solution of the Birkhoff-Rott equation (1.6) for $0 \leq t \leq T$, satisfying assumption (i) of Theorem 1.2. Then there exists a constant $c(m, M)>0$ such that if on some fixed interval $(a, b)$ there exists a $\delta_{0}>0$ such that for some determination of the multivalued function $\ln z_{\alpha}$ we have

$$
\sup _{[0, T]}\left\|\ln z_{\alpha}(\cdot, t)\right\|_{\mathrm{BMO}(a, b), \delta_{0}} \leq c(m, M),
$$

then $z_{\alpha} \in H^{1}(K)$ for every compact rectangle $K$ in $(a, b) \times(0, T)$. If, moreover, $\operatorname{Im}\left((1+i) \ln z_{\alpha}(\cdot, 0)\right) \in H_{\mathrm{loc}}^{1 / 2}(a, b)$, then $\operatorname{Re}\left((1+i) \ln z_{\alpha}(\cdot, 0)\right) \in H_{\mathrm{loc}}^{1 / 2}(a, b)$, $z_{\alpha}(\cdot, 0) \in H_{\mathrm{loc}}^{1 / 2}(a, b)$, and $z_{\alpha} \in H^{1}(K)$ for every compact rectangle $K$ in $(a, b) \times$ $[0, T)$.

We use a difference quotient argument to prove Proposition 3.3. Without loss of generality, we assume $(a, b)$ is a bounded interval. We first prove a lemma.

Lemma 3.4 Assume that $z: \mathbb{R} \rightarrow \mathbb{C}$ satisfies

$$
m|x-y| \leq|z(x)-z(y)| \leq M|x-y| \text { for all } x, y \in \mathbb{R},
$$

where $m, M>0$ are some constants. Assume further that $\left\|z^{\prime}\right\|_{\mathrm{BMO}(I), \delta_{0}} \leq \frac{1}{4}$ for some open interval $I$, and $\delta_{0}>0$. Let

$$
b(x)=\int \frac{1}{z(x)-z(y)} d y .
$$


Then there is a constant $k=k(m, M)>0$, depending only on $m$ and $M$ such that on any interval $[c, d] \subset I$,

$$
\|b\|_{\mathrm{BMO}(c, d), \delta_{1}} \leq k\left\|z^{\prime}\right\|_{\mathrm{BMO}(I), \delta_{0}}^{1 / 2},
$$

where $\delta_{1}=\min \left\{\delta_{0}\left\|z^{\prime}\right\|_{\mathrm{BMO}(I), \delta_{0}}^{1 / 2}, 2 \operatorname{dist}([c, d], \partial I)\left\|z^{\prime}\right\|_{\mathrm{BMO}(I), \delta_{0}}^{1 / 2}\right\}$.

PROOF: Let $Q \subset[c, d]$ be an interval with length $|Q| \leq \delta_{1}$ and $\bar{Q}=\lambda Q$, where $\lambda=\left\|z^{\prime}\right\|_{\mathrm{BMO}(I), \delta_{0}}^{-1 / 2}$. From our assumptions, we have $\lambda \geq 2, \bar{Q} \subset I$, and $|\bar{Q}|=\lambda|Q| \leq \delta_{0}$. Since p. v. $\int \frac{z^{\prime}(y)}{z(x)-z(y)} d y=0$, we have

$$
b(x)=\int \frac{1}{z(x)-z(y)} d y=\int \frac{1}{z(x)-z(y)}\left(1-\frac{1}{m_{\bar{Q}}\left(z^{\prime}\right)} z^{\prime}(y)\right) d y .
$$

Let $a(y)=1-\left[1 / m_{\bar{Q}}\left(z^{\prime}\right)\right] z^{\prime}(y)$. We have that $a \in L^{\infty}(\mathbb{R})$ and $\|a\|_{\infty} \leq 1+M / m$. Let $a_{1}=a \chi_{\bar{Q}}$ and $a_{2}=a-a_{1}$. Then

$$
b(x)=\int \frac{1}{z(x)-z(y)} a_{1}(y) d y+\int \frac{1}{z(x)-z(y)} a_{2}(y) d y=b_{1}(x)+b_{2}(x) .
$$

From the $L^{2}$ boundedness of the Cauchy integral,

$$
C_{1} f(x)=\int \frac{1}{z(x)-z(y)} f(y) d y
$$

(see Theorem 1.1 and $[5,11,13,17]$ ), there are constants $k_{1}$ and $k_{2}$ such that

$$
\begin{aligned}
& \frac{1}{|Q|} \int_{Q}\left|b_{1}(x)\right| d x \leq \frac{1}{|Q|^{1 / 2}}\left(\int_{Q}\left|b_{1}(x)\right|^{2} d x\right)^{1 / 2} \\
& \leq \frac{k_{1}}{|Q|^{1 / 2}}\left(\int\left|a_{1}(x)\right|^{2} d x\right)^{1 / 2} \leq \frac{k_{1}}{m|Q|^{1 / 2}}\left(\int_{\bar{Q}}\left|z^{\prime}(x)-m_{\bar{Q}}\left(z^{\prime}\right)\right|^{2} d x\right)^{1 / 2} \\
& \leq k_{2}\left\|z^{\prime}\right\|_{\mathrm{BMO}(I), \delta_{0}}\left(\frac{|\bar{Q}|}{|Q|}\right)^{1 / 2}=k_{2}\left\|z^{\prime}\right\|_{\mathrm{BMO}(I), \delta_{0}}^{3 / 4} \leq k_{2}\left\|z^{\prime}\right\|_{\mathrm{BMO}(I), \delta_{0}}^{1 / 2} .
\end{aligned}
$$

(In the last inequality above we used the assumption that $\left\|z^{\prime}\right\|_{\mathrm{BMO}(I), \delta_{0}} \leq \frac{1}{4}<1$.) 
Let $x_{0}$ be the center of the interval $Q$. For $b_{2}$, we have

$$
\begin{aligned}
& \frac{1}{|Q|} \int_{Q}\left|b_{2}(x)-b_{2}\left(x_{0}\right)\right| d x \\
& \leq \frac{1}{|Q|} \int_{Q}\left(\int_{\bar{Q}^{c}}\left|\frac{1}{z(x)-z(y)}-\frac{1}{z\left(x_{0}\right)-z(y)}\right||a(y)| d y\right) d x \\
& \leq \frac{2 M}{m^{2}}\left(1+\frac{M}{m}\right) \frac{1}{|Q|} \int_{Q} \int_{\bar{Q}^{c}} \frac{\left|x-x_{0}\right|}{\left|x_{0}-y\right|^{2}} d y d x \leq \frac{2 M}{m^{2}}\left(1+\frac{M}{m}\right) \frac{|Q|}{|\bar{Q}|} \\
& =\frac{2 M}{m^{2}}\left(1+\frac{M}{m}\right)\left\|z^{\prime}\right\|_{\mathrm{BMO}(I), \delta_{0}}^{1 / 2} .
\end{aligned}
$$

Therefore

$$
\frac{1}{|Q|} \int_{Q}\left|b(x)-b_{2}\left(x_{0}\right)\right| d x \leq\left(k_{2}+\frac{2 M}{m^{2}}\left(1+\frac{M}{m}\right)\right)\left\|z^{\prime}\right\|_{\mathrm{BMO}(I), \delta_{0}}^{1 / 2}
$$

for every $Q \subset[c, d]$ satisfying $|Q| \leq \delta_{1}$. This proves Lemma 3.4.

Assume that $z=z(\alpha, t)$ satisfies the assumptions of Proposition 3.3; we want to find the constant $c(m, M)$. We begin with $c(m, M) \leq \min \left\{r_{0} / 8, r_{1} / 8,1 /\left(32 C_{1}\right)\right.$, $m /(10 M)\}$, where $r_{0}$ is as in Theorem 2.3 and Corollary 2.4, and $r_{1}$ and $C_{1}$ are as in Proposition 2.7. From Lemma 3.4, we have that for $t \in[0, T]$ and any closed interval $[c, d] \subset(a, b),\left\|z_{t}(\cdot, t)\right\|_{\mathrm{BMO}(c, d), \delta_{1}} \leq k\left\|z_{\alpha}(\cdot, t)\right\|_{\mathrm{BMO}(a, b), \delta_{0}}^{1 / 2}$, where $k=$ $k(m, M)$ is a constant depending on $m$ and $M$ only, and $\delta_{1}$ is as defined in Lemma 3.4 .

Now for any given $[c, d] \subset(a, b)$, let $Q$ be any interval satisfying $2 Q \subset$ [c,d], $|2 Q| \leq \frac{1}{2} \delta_{1} \leq \frac{1}{4} \delta_{0}$; let $Q_{\delta}$ be the interval having the same center as $Q$ and length $2 \delta_{1}$. We know $Q_{\delta} \subset(a, b)$ and $\left|Q_{\delta}\right| \leq \delta_{0}$. Let $\eta, \rho \in C_{0}^{\infty}(\mathbb{R})$ be as in (3.2). Let $0<|h|<\frac{1}{2} \delta_{1}$. We want to estimate the $L_{\text {loc }}^{2}$ norm of $z_{\alpha h}=$ $\frac{1}{h}\left(z_{\alpha}(\alpha+h, t)-z_{\alpha}(\alpha, t)\right)$ and $z_{t h}=\frac{1}{h}\left(z_{t}(\alpha+h, t)-z_{t}(\alpha, t)\right)$; in the process, $\tilde{z}_{\alpha h}=$ $\frac{1}{h}\left(z_{\alpha}(\alpha, t)-z_{h}(\alpha, t)\right)$, where $z_{h}(\alpha, t)=\frac{1}{h}(z(\alpha+h, t)-z(\alpha, t))$ is also involved. Notice that it follows from our assumption that $\tilde{z}_{\alpha h}, z_{\alpha h}, z_{t h} \in L^{2}([c, d] \times[0, T])$ for each $h \neq 0$.

Taking the difference quotient with respect to $\alpha$ on both sides of (1.6), we obtain that for any $h, 0<|h|<\frac{1}{2} \delta_{1}$, and $\alpha, \alpha \in \frac{5}{4} Q$,

$$
\begin{aligned}
\bar{z}_{t h}(\alpha, t) & \\
= & \frac{1}{2 \pi i h} \int\left(\frac{1}{z(\alpha+h, t)-z(\beta+h, t)}-\frac{1}{z(\alpha, t)-z(\beta, t)}\right) \rho(\beta) d \beta \\
& +\frac{1}{2 \pi i} \int \frac{-z_{h}(\alpha, t)+z_{h}(\beta, t)}{(z(\alpha+h, t)-z(\beta+h, t))(z(\alpha, t)-z(\beta, t))}(1-\rho(\beta)) d \beta .
\end{aligned}
$$


For fixed $t \in[0, T]$, let $Z_{\alpha}(\alpha, t)$ be the reflective periodic extension of $z_{\alpha}(\alpha, t)$ from $\alpha \in Q_{\delta}$ to $\mathbb{R}$. For $\alpha \in \operatorname{supp} \eta \subset \frac{5}{4} Q, \beta \in 2 Q$, and $0<|h|<\frac{1}{2} \delta_{1}$, we have

$$
\begin{gathered}
z(\alpha, t)-z(\beta, t)=Z(\alpha, t)-Z(\beta, t), \quad z_{h}(\beta, t)=Z_{h}(\beta, t), \\
z(\alpha+h, t)-z(\beta+h, t)=Z(\alpha+h, t)-Z(\beta+h, t), \quad \text { etc. }
\end{gathered}
$$

it follows from Proposition 2.8 that $\left\|\ln z_{\alpha}\right\|_{\mathrm{BMO}(\mathbb{R})} \leq 8\left\|\ln z_{\alpha}\right\|_{\mathrm{BMO}(a, b), \delta_{0}}$. Moreover, $Z(\cdot, t)$ defines a chord-arc curve for each fixed $t \in[0, T]$.

We calculate the first term on the right-hand side of (3.19). Let $\alpha \in \frac{5}{4} Q$. We have

$$
\begin{aligned}
\frac{1}{h} \int & \left(\frac{1}{z(\alpha+h, t)-z(\beta+h, t)}-\frac{1}{z(\alpha, t)-z(\beta, t)}\right) \rho(\beta) d \beta \\
= & \frac{1}{h} \int\left(\frac{z_{\beta}(\beta+h, t)}{z(\alpha+h, t)-z(\beta+h, t)} \frac{1}{z_{\beta}(\beta+h, t)}\right. \\
& \left.\quad-\frac{z_{\beta}(\beta, t)}{z(\alpha, t)-z(\beta, t)} \frac{1}{z_{\beta}(\beta, t)}\right) \rho(\beta) d \beta \\
= & \int \frac{z_{\beta}(\beta+h, t)}{z(\alpha+h, t)-z(\beta+h, t)}\left(\frac{1}{z_{\beta}}\right)_{h} \rho(\beta) d \beta \\
& +\int\left(\frac{z_{\beta}(\beta+h, t)}{z(\alpha+h, t)-z(\beta+h, t)}-\frac{z_{\beta}(\beta, t)}{z(\alpha, t)-z(\beta, t)}\right) \\
& +\frac{1}{h}\left(\frac{1}{z_{\beta}(\beta, t)}-\frac{1}{z_{h}(\beta, t)}\right) \rho(\beta) d \beta \\
\quad & \quad \cdot\left(\frac{z_{\beta}(\beta+h, t)}{z(\alpha+h, t)-z(\beta+h, t)}-\frac{z_{\beta}(\beta, t)}{z(\alpha, t)-z(\beta, t)}\right) \\
= & \frac{1}{h} \int\left(\frac{1}{z_{h}(\beta, t)}-\frac{1}{z_{h}(\alpha, t)}\right) \rho(\beta) d \beta \\
= & \frac{1}{\alpha-\beta}\left(\frac{1}{\left.\left.z_{\beta}\right)_{h} \rho(\beta) h t\right)-z(\beta+h, t)}-\frac{z_{\beta}(\beta, t)}{z(\alpha, t)-z(\beta, t)}\right) \rho(\beta) d \beta \frac{1}{z_{h}(\alpha, t)}
\end{aligned}
$$

where

$$
\mathrm{I}=\int\left(\frac{z_{\beta}(\beta+h, t)}{z(\alpha+h, t)-z(\beta+h, t)}-\frac{1}{\alpha-\beta}\right)\left(\frac{1}{z_{\beta}}\right)_{h} \rho(\beta) d \beta
$$

some further calculation gives

$$
\begin{aligned}
\mathrm{II}= & \int\left(\frac{z_{\beta}(\beta+h, t)}{z(\alpha+h, t)-z(\beta+h, t)}-\frac{z_{\beta}(\beta, t)}{z(\alpha, t)-z(\beta, t)}\right) \frac{-\tilde{z}_{\beta h}(\beta, t)}{z_{\beta}(\beta, t) z_{h}(\beta, t)} \rho(\beta) d \beta, \\
\mathrm{III}= & \int \frac{z_{\beta h}(\beta, t) \rho(\beta)}{z(\alpha+h, t)-z(\beta+h, t)}\left(\frac{1}{z_{h}(\beta, t)}-\frac{1}{z_{h}(\alpha, t)}\right) d \beta \\
& +\int \frac{z_{\beta}(\beta, t)\left(z_{h}(\beta, t)-z_{h}(\alpha, t)\right)}{(z(\alpha, t)-z(\beta, t))(z(\alpha+h, t)-z(\beta+h, t))}\left(\frac{1}{z_{h}(\beta, t)}-\frac{1}{z_{h}(\alpha, t)}\right) \rho(\beta) d \beta,
\end{aligned}
$$


and from integration by parts,

$$
\begin{aligned}
\mathrm{IV} & =\frac{1}{h z_{h}(\alpha, t)} \int \ln \frac{z(\alpha+h, t)-z(\beta+h, t)}{z(\alpha, t)-z(\beta, t)} \rho^{\prime}(\beta) d \beta \\
& =\int \frac{1}{h z_{h}(\alpha, t)}\left\{\int_{0}^{h} \frac{z_{\alpha}(\alpha+r, t)-z_{\beta}(\beta+r, t)}{z(\alpha+r, t)-z(\beta+r, t)} d r\right\} \rho^{\prime}(\beta) d \beta .
\end{aligned}
$$

Define

$$
\begin{aligned}
G_{1}(\alpha, t)= & \frac{1}{2 \pi i} \int \frac{-z_{h}(\alpha, t)+z_{h}(\beta, t)}{(z(\alpha+h, t)-z(\beta+h, t))(z(\alpha, t)-z(\beta, t))}(1-\rho(\beta)) d \beta \\
& +\frac{1}{2 \pi i}(\mathrm{I}+\mathrm{II}+\mathrm{III}+\mathrm{IV}) .
\end{aligned}
$$

From (3.19) and (3.21) we get

$$
\bar{z}_{t h}(\alpha, t)=\frac{1}{2 \pi i} \int \frac{1}{\alpha-\beta}\left(\frac{1}{z_{\beta}}\right)_{h} \rho(\beta) d \beta+G_{1}(\alpha, t) .
$$

Now multiplying $\eta(\alpha)$ on both sides of (3.22), we obtain

$$
\begin{aligned}
\eta(\alpha) \bar{z}_{t h}(\alpha, t)= & \frac{1}{2 \pi i} \int \frac{\eta(\beta)}{\alpha-\beta}\left(\frac{1}{z_{\beta}}\right)_{h} d \beta+\frac{1}{2 i}[\eta, H]\left\{\rho\left(\frac{1}{z_{\alpha}}\right)_{h}\right\} \\
& +\eta(\alpha) G_{1}(\alpha, t) .
\end{aligned}
$$

We first estimate $\int\left|\eta(\alpha) G_{1}(\alpha, t)\right|^{2} d \alpha$ for fixed $t \in[0, T]$. Replace $z(\alpha, t)$ by its reflective periodic extension $Z(\alpha, t)$ when necessary; notice that we can do so because of (3.20) and because we calculate only for $\alpha, \alpha+h \in Q_{\delta}$ and $\beta, \beta+h \in Q_{\delta}$. Apply Corollary 2.4 to $\eta(\alpha) \mathrm{I}$ and $\eta(\alpha) \mathrm{II}$; apply Proposition 2.7 and Theorem 2.6 to the first term of $\eta(\alpha)$ III; for the second term of $\eta(\alpha)$ III, notice that for $\alpha \in \operatorname{supp} \eta \subset \frac{5}{4} Q$,

$$
\begin{aligned}
\mid \int & \frac{z_{\beta}(\beta, t)\left(z_{h}(\beta, t)-z_{h}(\alpha, t)\right)}{(z(\alpha, t)-z(\beta, t))(z(\alpha+h, t)-z(\beta+h, t))}\left(\frac{1}{z_{h}(\beta, t)}-\frac{1}{z_{h}(\alpha, t)}\right) \rho(\beta) d \beta \mid \\
\leq & \frac{M}{m^{4}} \int \frac{\left|-z_{h}(\beta, t)+z_{h}(\alpha, t)\right|^{2}}{(\alpha-\beta)^{2}} \rho(\beta) d \beta \\
= & -\frac{M}{m^{4}} \int \frac{\left|-z_{h}(\beta, t)+z_{h}(\alpha, t)\right|^{2}}{\alpha-\beta} \rho^{\prime}(\beta) d \beta \\
& +2 \operatorname{Re} \frac{M}{m^{4}} \int \frac{-z_{h}(\beta, t)+z_{h}(\alpha, t)}{\alpha-\beta} \bar{z}_{\beta h}(\beta, t) \rho(\beta) d \beta \\
\leq & \frac{C}{|Q|}+2 \operatorname{Re} \frac{M}{m^{4}} \int \frac{-z_{h}(\beta, t)+z_{h}(\alpha, t)}{\alpha-\beta} \bar{z}_{\beta h}(\beta, t) \rho(\beta) d \beta=\mathrm{III}_{1}+\mathrm{III}_{2}
\end{aligned}
$$

for some constant $C$. We apply Proposition 2.7 and Theorem 2.6 to the second term $\mathrm{III}_{2}$. Furthermore, for $\alpha \in \frac{5}{4} Q$, we have $|\mathrm{IV}| \leq C /|Q|$ for some constant $C$. Therefore for fixed $t \in[0, T]$, there are constants $c_{1}$ and $c_{2}$, independent of $h, t$, $\delta_{0}$, and $\delta_{1}$, such that 


$$
\begin{aligned}
& \int|\eta(\alpha)|^{2}\left|G_{1}(\alpha, t)\right|^{2} d \alpha \\
& \leq c_{1}\left\|\ln z_{\alpha}\right\|_{\mathrm{BMO}(a, b), \delta_{0}}^{2}\left(\int\left|\rho(\beta) z_{\beta h}\right|^{2} d \beta+\int\left|\rho(\beta) \tilde{z}_{\beta h}\right|^{2} d \beta\right)+\frac{c_{2}}{|Q|} .
\end{aligned}
$$

For the second term on the right-hand side of (3.23), we have the following lemma:

LEMMA 3.5 There is a constant $c_{3}$ such that

$$
\left\|[\eta, H]\left\{\rho\left(\frac{1}{z_{\alpha}}\right)_{h}\right\}\right\|_{L^{2}(\mathbb{R})} \leq \frac{c_{3}}{|Q|^{1 / 2}} .
$$

PROOF: We have

$$
\begin{aligned}
{[\eta, H] } & \left\{\rho\left(\frac{1}{z_{\alpha}}\right)_{h}\right\}(\alpha, t) \\
= & \frac{1}{h} \int \frac{\eta(\alpha)-\eta(\beta)}{\alpha-\beta}\left(\frac{1}{z_{\beta}(\beta+h, t)}-\frac{1}{z_{\beta}(\beta, t)}\right) \rho(\beta) d \beta \\
= & \frac{1}{h} \int\left(\frac{\eta(\alpha)-\eta(\beta-h)}{\alpha-\beta+h}-\frac{\eta(\alpha)-\eta(\beta)}{\alpha-\beta}\right) \frac{1}{z_{\beta}(\beta, t)} \rho(\beta) d \beta \\
& +\int \frac{\eta(\alpha)-\eta(\beta)}{\alpha-\beta} \frac{1}{h}(\rho(\beta)-\rho(\beta+h)) \frac{1}{z_{\beta}(\beta+h, t)} d \beta
\end{aligned}
$$

where

$$
\begin{aligned}
\frac{1}{h} \int & \left(\frac{\eta(\alpha)-\eta(\beta-h)}{\alpha-\beta+h}-\frac{\eta(\alpha)-\eta(\beta)}{\alpha-\beta}\right) \frac{1}{z_{\beta}(\beta, t)} \rho(\beta) d \beta \\
= & \frac{1}{h} \iint_{0}^{h}\left(-\frac{\eta(\alpha)-\eta(\beta-r)}{(\alpha-\beta+r)^{2}}+\frac{\eta^{\prime}(\beta-r)}{\alpha-\beta+r}\right) d r \frac{1}{z_{\beta}(\beta, t)} \rho(\beta) d \beta \\
= & \frac{1}{h} \iint_{0}^{h}\left(-\frac{\eta(\alpha)-\eta(\beta)}{(\alpha-\beta)^{2}}+\frac{\eta^{\prime}(\beta)}{\alpha-\beta}\right) \frac{1}{z_{\beta}(\beta+r, t)} \rho(\beta+r) d r d \beta .
\end{aligned}
$$

Lemma 3.5 follows from Corollary 2.5 and the $L^{2}$ boundedness of the Hilbert transform $H$.

Finally, let's calculate

$$
\begin{aligned}
\int \mid \eta(\alpha) \bar{z}_{t h}(\alpha, t)-\frac{1}{2 \pi i} \int \frac{\eta(\beta)}{\alpha-\beta} & \left.\left(\frac{1}{z_{\beta}}\right)_{h} d \beta\right|^{2} d \alpha \\
& =\int\left|\eta(\alpha) \bar{z}_{t h}(\alpha, t)-\frac{1}{2 i} H\left(\eta\left(\frac{1}{z_{\alpha}}\right)_{h}\right)\right|^{2} d \alpha
\end{aligned}
$$


We have

$$
\begin{aligned}
\int\left|\eta(\alpha) \bar{z}_{t h}(\alpha, t)-\frac{1}{2 i} H\left(\eta\left(\frac{1}{z_{\alpha}}\right){ }_{h}\right)\right|^{2} d \alpha \\
=\int\left|\eta(\alpha) z_{t h}\right|^{2} d \alpha+\frac{1}{4} \int\left|H\left(\eta\left(\frac{1}{z_{\alpha}}\right)_{h}\right)\right|^{2} d \alpha \\
\quad-\frac{1}{\pi} \operatorname{Im} \iint \frac{\eta(\alpha) \eta(\beta) z_{t h}(\alpha, t)\left(1 / z_{\beta}\right)_{h}(\beta, t)}{\alpha-\beta} d \alpha d \beta,
\end{aligned}
$$

where

$$
\begin{aligned}
- & \iint \frac{\eta(\alpha) \eta(\beta) z_{t h}(\alpha, t)\left(1 / z_{\beta}\right)_{h}(\beta, t)}{\alpha-\beta} d \alpha d \beta \\
= & \iint \frac{\eta(\alpha) \eta(\beta) z_{t h}(\alpha, t) z_{\beta h}(\beta, t)}{(\alpha-\beta) z_{h}^{2}(\beta, t)} d \alpha d \beta \\
& \quad+\iint \frac{\eta(\alpha) \eta(\beta) z_{t h}(\alpha, t) z_{\beta h}(\beta, t)}{\alpha-\beta}\left(\frac{1}{z_{\beta}(\beta, t) z_{\beta}(\beta+h, t)}-\frac{1}{z_{h}^{2}(\beta, t)}\right) d \alpha d \beta \\
= & \mathrm{I}+\mathrm{II} .
\end{aligned}
$$

Using integration by parts, symmetry, antisymmetry, and interchange of the variables $\alpha$ and $\beta$, we have for the first term I of (3.26),

$$
\begin{aligned}
\mathrm{I}= & -\iint \frac{\eta(\alpha) \eta(\beta) z_{t h}(\alpha, t)}{\alpha-\beta} \partial_{\beta}\left(\frac{1}{z_{h}(\beta, t)}-\frac{1}{z_{h}(\alpha, t)}\right) d \alpha d \beta \\
= & \iint \frac{\eta(\alpha) \eta(\beta) z_{t h}(\alpha, t)}{(\alpha-\beta)^{2}}\left(\frac{1}{z_{h}(\beta, t)}-\frac{1}{z_{h}(\alpha, t)}\right) d \alpha d \beta \\
& +\iint \frac{\eta(\alpha) \eta^{\prime}(\beta) z_{t h}(\alpha, t)}{\alpha-\beta}\left(\frac{1}{z_{h}(\beta, t)}-\frac{1}{z_{h}(\alpha, t)}\right) d \alpha d \beta \\
= & \frac{1}{4} \iint \frac{\eta(\alpha) \eta(\beta) \partial_{t}\left(z_{h}(\alpha, t)-z_{h}(\beta, t)\right)^{2}}{(\alpha-\beta)^{2} z_{h}(\alpha, t) z_{h}(\beta, t)} d \alpha d \beta \\
& +\int \eta(\alpha) z_{t h}(\alpha, t) \int \frac{\eta^{\prime}(\beta)}{\alpha-\beta}\left(\frac{1}{z_{h}(\beta, t)}-\frac{1}{z_{h}(\alpha, t)}\right) d \beta d \alpha \\
= & \frac{1}{4} \frac{d}{d t} \iint \frac{\eta(\alpha) \eta(\beta)\left(z_{h}(\alpha, t)-z_{h}(\beta, t)\right)^{2}}{(\alpha-\beta)^{2} z_{h}(\alpha, t) z_{h}(\beta, t)} d \alpha d \beta \\
& +\frac{1}{2} \iint \frac{\eta(\alpha) \eta(\beta)\left(z_{h}(\alpha, t)-z_{h}(\beta, t)\right)^{2} z_{t h}(\alpha, t)}{(\alpha-\beta)^{2} z_{h}^{2}(\alpha, t) z_{h}(\beta, t)} d \alpha d \beta \\
& +\int \eta(\alpha) z_{t h}(\alpha, t) \int \frac{\eta^{\prime}(\beta)}{\alpha-\beta}\left(\frac{1}{z_{h}(\beta, t)}-\frac{1}{z_{h}(\alpha, t)}\right) d \beta d \alpha \\
= & \mathrm{I}_{1}+\mathrm{I}_{2}+\mathrm{I}_{3} .
\end{aligned}
$$


For the second term II in (3.26), we again interchange variables $\alpha$ and $\beta$ and use the antisymmetry of $\frac{1}{\alpha-\beta}$; we get

$$
\begin{aligned}
\mathrm{II}= & \iint \frac{\eta(\alpha) \eta(\beta) z_{t h}(\alpha, t) z_{\beta h}(\beta, t)}{\alpha-\beta}\left(\frac{1}{z_{\beta}(\beta, t) z_{\beta}(\beta+h, t)}-\frac{1}{z_{h}^{2}(\beta, t)}\right) d \alpha d \beta \\
= & \frac{1}{2} \iint \eta(\alpha) \eta(\beta) \frac{z_{t h}(\alpha, t) z_{\beta h}(\beta, t)-z_{t h}(\beta, t) z_{\alpha h}(\alpha, t)}{\alpha-\beta} \\
& \quad \cdot\left(\frac{1}{z_{\beta}(\beta, t) z_{\beta}(\beta+h, t)}-\frac{1}{z_{h}^{2}(\beta, t)}\right) d \alpha d \beta \\
& +\frac{1}{2} \iint \eta(\alpha) \eta(\beta) \frac{z_{t h}(\beta, t) z_{\alpha h}(\alpha, t)}{\alpha-\beta}\left(\frac{1}{z_{\beta}(\beta, t) z_{\beta}(\beta+h, t)}-\frac{1}{z_{\alpha}(\alpha, t) z_{\alpha}(\alpha+h, t)}\right) d \alpha d \beta \\
+ & \frac{1}{2} \iint \eta(\alpha) \eta(\beta) \frac{z_{t h}(\beta, t) z_{\alpha h}(\alpha, t)}{\alpha-\beta}\left(\frac{1}{z_{h}^{2}(\alpha, t)}-\frac{1}{z_{h}^{2}(\beta, t)}\right) d \alpha d \beta=\mathrm{II}_{1}+\mathrm{II}_{2}+\mathrm{II}_{3} .
\end{aligned}
$$

Furthermore,

$$
\mathrm{I}_{2}=\frac{1}{2} \int \eta(\alpha) \frac{z_{t h}(\alpha, t)}{z_{h}^{2}(\alpha, t)} \int \frac{\eta(\beta)\left(z_{h}(\alpha, t)-z_{h}(\beta, t)\right)^{2}}{(\alpha-\beta)^{2} z_{h}(\beta, t)} d \beta d \alpha
$$

where

$$
\begin{aligned}
\mid \int & \frac{\eta(\beta)\left(z_{h}(\alpha, t)-z_{h}(\beta, t)\right)^{2}}{(\alpha-\beta)^{2} z_{h}(\beta, t)} d \beta \mid \\
\leq & \frac{1}{m} \int \frac{\eta(\beta)\left|z_{h}(\alpha, t)-z_{h}(\beta, t)\right|^{2}}{(\alpha-\beta)^{2}} d \beta \\
= & -\frac{1}{m} \int \frac{\eta^{\prime}(\beta)\left|z_{h}(\alpha, t)-z_{h}(\beta, t)\right|^{2}}{\alpha-\beta} d \beta \\
& +\frac{2}{m} \operatorname{Re} \int \frac{\eta(\beta)\left(z_{h}(\alpha, t)-z_{h}(\beta, t)\right) \overline{z_{\beta h}(\beta, t)}}{\alpha-\beta} d \beta,
\end{aligned}
$$

while

$$
\begin{aligned}
\mathrm{II}_{1}= & \frac{1}{2} \iint \eta(\alpha) \eta(\beta) \frac{z_{t h}(\alpha, t)\left(h z_{\beta h}(\beta, t)-h z_{\alpha h}(\alpha, t)\right)}{\alpha-\beta} \frac{1}{h}\left(\frac{1}{z_{\beta}(\beta, t) z_{\beta}(\beta+h, t)}-\frac{1}{z_{h}^{2}(\beta, t)}\right) d \alpha d \beta \\
& +\frac{1}{2} \iint \eta(\alpha) \eta(\beta) \frac{z_{\alpha h}(\alpha, t)\left(h z_{t h}(\alpha, t)-h z_{t h}(\beta, t)\right)}{\alpha-\beta} \frac{1}{h}\left(\frac{1}{z_{\beta}(\beta, t) z_{\beta}(\beta+h, t)}-\frac{1}{z_{h}^{2}(\beta, t)}\right) d \alpha d \beta .
\end{aligned}
$$

Notice that $h z_{\alpha h}(\alpha, t)=z_{\alpha}(\alpha+h, t)-z_{\alpha}(\alpha, t)$, and $h z_{t h}(\alpha, t)=z_{t}(\alpha+h, t)-$ $z_{t}(\alpha, t)$. Summing up the above calculations and applying the results in Section 2 
and Lemma 3.4, we conclude that there are constants $c_{4}, c_{5}, c_{6}$, and $c_{7}$ such that

$$
\begin{aligned}
\frac{1}{\pi} \operatorname{Im} & \iint \frac{\eta(\alpha) \eta(\beta) z_{t h}(\alpha, t)\left(1 / z_{\beta}\right)_{h}(\beta, t)}{\alpha-\beta} d \alpha d \beta \\
\leq & -\frac{1}{4 \pi} \frac{d}{d t} \operatorname{Im} \iint \frac{\eta(\alpha) \eta(\beta)\left(z_{h}(\alpha, t)-z_{h}(\beta, t)\right)^{2}}{(\alpha-\beta)^{2} z_{h}(\alpha, t) z_{h}(\beta, t)} d \alpha d \beta \\
& +\frac{c_{4}}{|Q|^{1 / 2}}\left\|\eta z_{t h}\right\|_{2}+c_{5}\left\|\ln z_{\alpha}\right\|_{\mathrm{BMO}(a, b), \delta_{0}}\left\|\eta z_{t h}\right\|_{2}\left(\left\|\eta z_{\alpha h}\right\|_{2}+\left\|\eta \tilde{z}_{\alpha h}\right\|_{2}\right) \\
& +c_{6}\left\|z_{t}(\cdot, t)\right\|_{\mathrm{BMO}(c, d), \delta_{1}}\left\|\eta z_{\alpha h}\right\|_{2}\left(\left\|\eta z_{\alpha h}\right\|_{2}+\left\|\eta \tilde{z}_{\alpha h}\right\|_{2}\right) \\
\leq & -\frac{1}{4 \pi} \frac{d}{d t} \operatorname{Im} \iint \frac{\eta(\alpha) \eta(\beta)\left(z_{h}(\alpha, t)-z_{h}(\beta, t)\right)^{2}}{(\alpha-\beta)^{2} z_{h}(\alpha, t) z_{h}(\beta, t)} d \alpha d \beta \\
& +\frac{1}{2}\left\|\eta z_{t h}\right\|_{2}^{2}+\frac{c_{4}^{2}}{|Q|} \\
& +\left(2 c_{5}^{2}\left\|\ln z_{\alpha}\right\|_{\mathrm{BMO}(a, b), \delta_{0}}^{2}+c_{7}\left\|\ln z_{\alpha}\right\|_{\mathrm{BMO}(a, b), \delta_{0}}^{1 / 2}\right)\left(\left\|\eta z_{\alpha h}\right\|_{2}^{2}+\left\|\eta \tilde{z}_{\alpha h}\right\|_{2}^{2}\right) .
\end{aligned}
$$

This together with (3.25) gives that

$$
\begin{aligned}
& \int\left|\eta(\alpha) \bar{z}_{t h}(\alpha, t)-\frac{1}{2 i} H\left(\eta\left(\frac{1}{z_{\alpha}}\right)_{h}\right)\right|^{2} d \alpha \\
& \geq \frac{1}{2} \int\left|\eta(\alpha) z_{t h}\right|^{2} d \alpha+\frac{1}{4 M^{4}} \int\left|\eta(\alpha) z_{\alpha h}\right|^{2} d \alpha \\
& \quad+\frac{1}{4 \pi} \frac{d}{d t} \operatorname{Im} \iint \frac{\eta(\alpha) \eta(\beta)\left(z_{h}(\alpha, t)-z_{h}(\beta, t)\right)^{2}}{(\alpha-\beta)^{2} z_{h}(\alpha, t) z_{h}(\beta, t)} d \alpha d \beta-\frac{c_{4}^{2}}{|Q|} \\
& \quad-\left(2 c_{5}^{2}\left\|\ln z_{\alpha}\right\|_{\mathrm{BMO}(a, b), \delta_{0}}^{2}+c_{7}\left\|\ln z_{\alpha}\right\|_{\mathrm{BMO}(a, b), \delta_{0}}^{1 / 2}\right)\left(\left\|\eta z_{\alpha h}\right\|_{2}^{2}+\left\|\eta \tilde{z}_{\alpha h}\right\|_{2}^{2}\right) .
\end{aligned}
$$

From (3.23), combining (3.24), Lemma 3.5, and (3.28), we conclude that there are constants $c_{1}, c_{2}$, and $c_{3}$, depending only on $M$ and $m$ such that for $t \in[0, T]$ and $0<|h|<\frac{1}{2} \delta_{1}$,

$$
\begin{aligned}
\frac{1}{2} \int \mid & \left.\eta(\alpha) z_{t h}(\alpha, t)\right|^{2} d \alpha+\frac{1}{4 M^{4}} \int\left|\eta(\alpha) z_{\alpha h}(\alpha, t)\right|^{2} d \alpha \\
\leq & -\frac{1}{4 \pi} \frac{d}{d t} \operatorname{Im} \iint \frac{\eta(\alpha) \eta(\beta)\left(z_{h}(\alpha, t)-z_{h}(\beta, t)\right)^{2}}{(\alpha-\beta)^{2} z_{h}(\alpha, t) z_{h}(\beta, t)} d \alpha d \beta+\frac{c_{3}}{|Q|} \\
& +\left(c_{1}\left\|\ln z_{\alpha}\right\|_{\mathrm{BMO}(a, b), \delta_{0}}^{2}+c_{2}\left\|\ln z_{\alpha}\right\|_{\mathrm{BMO}(a, b), \delta_{0}}^{1 / 2}\right) \\
& \cdot \int_{2 Q}\left(\left|z_{\alpha h}(\alpha, t)\right|^{2}+\left|\tilde{z}_{\alpha h}(\alpha, t)\right|^{2}\right) d \alpha .
\end{aligned}
$$




\subsection{Interior $H^{1}$ Regularity of $z_{\alpha}$}

Taking $\delta(t)=t^{2}(T-t)^{2}$ for $t \in[0, T]$ and $\delta(t)=0$ for $t \notin[0, T]$ as in the heuristic argument, and using integration by parts, we get

$$
\begin{aligned}
& \frac{1}{2} \int \delta(t) \int\left|\eta(\alpha) z_{t h}(\alpha, t)\right|^{2} d \alpha d t \\
& +\frac{1}{4 M^{4}} \int \delta(t) \int\left|\eta(\alpha) z_{\alpha h}(\alpha, t)\right|^{2} d \alpha d t \\
& \leq \frac{1}{4 \pi} \operatorname{Im} \int \delta^{\prime}(t) \iint \frac{\eta(\alpha) \eta(\beta)\left(z_{h}(\alpha, t)-z_{h}(\beta, t)\right)^{2}}{(\alpha-\beta)^{2} z_{h}(\alpha, t) z_{h}(\beta, t)} d \alpha d \beta d t \\
& \quad+\frac{c_{3}}{|Q|} \int \delta(t) d t \\
& +\left(c_{1} \sup _{[0, T]}\left\|\ln z_{\alpha}\right\|_{*, \delta_{0}}^{2}+c_{2} \sup _{[0, T]}\left\|\ln z_{\alpha}\right\|_{*, \delta_{0}}^{1 / 2}\right) \\
& \quad \cdot \int \delta(t) \int_{2 Q}\left(\left|z_{\alpha h}(\alpha, t)\right|^{2}+\left|\tilde{z}_{\alpha h}(\alpha, t)\right|^{2}\right) d \alpha d t .
\end{aligned}
$$

From the fact that $\delta^{\prime}(t)^{2} \leq 4 T^{2} \delta(t)$ for $t \in[0, T]$, integration by parts, the Hölder inequality, and Theorem 2.6, we have

$$
\begin{aligned}
\frac{1}{4 \pi} \mid & \operatorname{Im} \int \delta^{\prime}(t) \iint \frac{\eta(\alpha) \eta(\beta)\left(z_{h}(\alpha, t)-z_{h}(\beta, t)\right)^{2}}{(\alpha-\beta)^{2} z_{h}(\alpha, t) z_{h}(\beta, t)} d \alpha d \beta d t \mid \\
\leq & \frac{1}{4 \pi m^{2}} \int\left|\delta^{\prime}(t)\right| \int \eta(\alpha)\left|\int \frac{\eta^{\prime}(\beta)\left|z_{h}(\alpha, t)-z_{h}(\beta, t)\right|^{2}}{\alpha-\beta} d \beta\right| d \alpha d t \\
& +\frac{1}{2 \pi m^{2}} \int\left|\delta^{\prime}(t)\right|\left|\int \eta(\alpha) \int \frac{\eta(\beta)}{\alpha-\beta}\left(z_{h}(\alpha, t)-z_{h}(\beta, t)\right) \overline{z_{\beta h}(\beta, t)} d \beta d \alpha\right| d t \\
\leq & k_{1}+k_{2}|Q|+\frac{1}{8 M^{4}} \iint \delta(t)\left|\eta(\alpha) z_{\alpha h}(\alpha, t)\right|^{2} d \alpha d t<\infty,
\end{aligned}
$$

where $k_{1}$ and $k_{2}$ are constants depending on $T, m$, and $M$. Therefore

$$
\begin{aligned}
& \frac{1}{2} \int \delta(t) \int\left|\eta(\alpha) z_{t h}(\alpha, t)\right|^{2} d \alpha d t+\frac{1}{8 M^{4}} \int \delta(t) \int\left|\eta(\alpha) z_{\alpha h}(\alpha, t)\right|^{2} d \alpha d t \\
& \leq\left(c_{1} \sup _{[0, T]}\left\|\ln z_{\alpha}\right\|_{*, \delta_{0}}^{2}+c_{2} \sup _{[0, T]}\left\|\ln z_{\alpha}\right\|_{*, \delta_{0}}^{1 / 2}\right) \\
& \quad \cdot \int \delta(t) \int_{2 Q}\left(\left|z_{\alpha h}(\alpha, t)\right|^{2}+\left|\tilde{z}_{\alpha h}(\alpha, t)\right|^{2}\right) d \alpha d t+c_{4}|Q|+\frac{c_{5}}{|Q|}
\end{aligned}
$$


for some constants $c_{1}, c_{2}, c_{4}$, and $c_{5}$. Recalling $\eta(\alpha)=1$ for all $\alpha \in Q$, we then obtain

$$
\begin{aligned}
\frac{1}{2} \int \delta(t) \int_{Q}\left|z_{t h}(\alpha, t)\right|^{2} d \alpha d t+\frac{1}{8 M^{4}} \int \delta(t) \int_{Q}\left|z_{\alpha h}(\alpha, t)\right|^{2} d \alpha d t \\
\leq\left(c_{1} \sup _{[0, T]}\left\|\ln z_{\alpha}\right\|_{*, \delta_{0}}^{2}+c_{2} \sup _{[0, T]}\left\|\ln z_{\alpha}\right\|_{*, \delta_{0}}^{1 / 2}\right) \\
\quad \cdot \int \delta(t) \int_{2 Q}\left(\left|z_{\alpha h}(\alpha, t)\right|^{2}+\left|\tilde{z}_{\alpha h}(\alpha, t)\right|^{2}\right) d \alpha d t+c_{4}|Q|+\frac{c_{5}}{|Q|} .
\end{aligned}
$$

Now let's study the term $\int_{2 Q}\left|\tilde{z}_{\alpha h}(\alpha, t)\right|^{2} d \alpha$. For a set $S, S=Q$ or $S=2 Q$, we have

$$
\begin{aligned}
|h|^{3 / 2} \int_{S}\left|\tilde{z}_{\alpha h}(\alpha, t)\right|^{2} d \alpha & =|h|^{-1 / 2} \int_{S}\left|z_{\alpha}(\alpha, t)-z_{h}(\alpha, t)\right|^{2} d \alpha \\
& =|h|^{-1 / 2} \int_{S}\left|\frac{1}{h} \int_{0}^{h}\left(z_{\alpha}(\alpha, t)-z_{\alpha}(\alpha+s, t)\right) d s\right|^{2} d \alpha \\
& \leq|h|^{-3 / 2} \int_{S}\left|\int_{0}^{h}\right| z_{\alpha}(\alpha, t)-\left.z_{\alpha}(\alpha+s, t)\right|^{2} d s \mid d \alpha
\end{aligned}
$$

Therefore for $l>0$,

$$
\begin{aligned}
& \int_{0}^{l} h^{3 / 2} \int_{S}\left|\tilde{z}_{\alpha h}(\alpha, t)\right|^{2} d \alpha d h \\
& \quad \leq \int_{0}^{l} h^{-3 / 2} \iint_{S}^{h}\left|z_{\alpha}(\alpha, t)-z_{\alpha}(\alpha+s, t)\right|^{2} d s d \alpha d h \\
& \quad=\int_{0}^{l} \int_{S}\left|z_{\alpha}(\alpha, t)-z_{\alpha}(\alpha+s, t)\right|^{2}\left(\int_{s}^{l} h^{-3 / 2} d h\right) d s d \alpha \\
& \quad=2 \int_{0}^{l} \int_{S}\left(\frac{1}{s^{1 / 2}}-\frac{1}{l^{1 / 2}}\right) s^{2}\left|z_{\alpha s}(\alpha, t)\right|^{2} d \alpha d s \\
& \quad \leq 2 \int_{0}^{l} h^{3 / 2} \int_{S}\left|z_{\alpha h}(\alpha, t)\right|^{2} d \alpha d h .
\end{aligned}
$$

For the same reason, if $l<0$,

$$
\int_{l}^{0}|h|^{3 / 2} \int_{S}\left|\tilde{z}_{\alpha h}(\alpha, t)\right|^{2} d \alpha d h \leq 2 \int_{l}^{0}|h|^{3 / 2} \int_{S}\left|z_{\alpha h}(\alpha, t)\right|^{2} d \alpha d h .
$$


Let

$$
\begin{aligned}
& U_{[\alpha l]}(\alpha, t)=\frac{\operatorname{sign} l}{|l|^{5 / 2}} \int_{0}^{l}|h|^{3 / 2}\left|z_{\alpha h}(\alpha, t)\right|^{2} d h, \\
& U_{[t l]}(\alpha, t)=\frac{\operatorname{sign} l}{|l|^{5 / 2}} \int_{0}^{l}|h|^{3 / 2}\left|z_{t h}(\alpha, t)\right|^{2} d h .
\end{aligned}
$$

We multiply each term in (3.31) by $|h|^{3 / 2}$ and then integrate with respect to $h$ from 0 to $l$ if $l>0$, and from $l$ to 0 if $l<0$; we get for $0<|l|<\frac{1}{2} \delta_{1}$,

$$
\begin{aligned}
& \frac{1}{2} \int \delta(t) \int_{Q} U_{[t l]}(\alpha, t) d \alpha d t+\frac{1}{8 M^{4}} \int \delta(t) \int_{Q} U_{[\alpha l]}(\alpha, t) d \alpha d t \\
& \leq 3\left(c_{1} \sup _{[0, T]}\left\|\ln z_{\alpha}\right\|_{*, \delta_{0}}^{2}+c_{2} \sup _{[0, T]}\left\|\ln z_{\alpha}\right\|_{*, \delta_{0}}^{1 / 2}\right) \int \delta(t) \int_{2 Q} U_{[\alpha l]}(\alpha, t) d \alpha d t \\
& \quad+c_{4}|Q|+\frac{c_{5}}{|Q|}
\end{aligned}
$$

Now take

$$
c(m, M)=\min \left\{\frac{1}{\sqrt{600 M^{4} c_{1}}}, \frac{1}{\left(600 M^{4} c_{2}\right)^{2}}, \frac{r_{0}}{8}, \frac{r_{1}}{8}, \frac{1}{32 C_{1}}, \frac{m}{10 M}\right\}
$$

where $r_{0}$ is as in Theorem 2.3 and Corollary 2.4, and $r_{1}$ and $C_{1}$ are as in Proposition 2.7. We have from (3.33) that for $0<|l|<\frac{1}{2} \delta_{1}$,

$$
\begin{aligned}
\frac{1}{2} \int \delta(t) \int_{Q} U_{[t]]}(\alpha, t) d \alpha d t+\frac{1}{8 M^{4}} \int \delta(t) \int_{Q} U_{[\alpha l]}(\alpha, t) d \alpha d t \\
\leq \frac{1}{100 M^{4}} \int \delta(t) \int_{2 Q} U_{[\alpha l]}(\alpha, t) d \alpha d t \\
\quad+c_{4}|Q|+\frac{c_{5}}{|Q|} .
\end{aligned}
$$

Notice that (3.35) is similar to (3.13); it holds for all intervals $Q$ satisfying $2 Q \subset$ $[c, d]$ and $|2 Q| \leq \delta_{1}$.

Now let $I \subset[c, d]$ be any closed interval that satisfies $|I| \leq \frac{1}{2} \delta_{1}$. Notice that for each fixed $l, 0<|l|<\frac{1}{2} \delta_{1}$,

$$
\int \delta(t) \int_{I} U_{[\alpha l]}(\alpha, t) d \alpha d t<\infty
$$


It follows from the same argument as in Proposition 3.2 that there is a constant $K$, independent of $l$, such that for $0<|l|<\frac{1}{2} \delta_{1}$,

$$
\begin{aligned}
& \frac{1}{2} \int \delta(t) \int_{I} \operatorname{dist}^{2}(\alpha, \partial I) U_{[t l]}(\alpha, t) d \alpha d t \\
& \quad+\frac{1}{16 M^{4}} \int \delta(t) \int_{I} \operatorname{dist}^{2}(\alpha, \partial I) U_{[\alpha l]}(\alpha, t) d \alpha d t \leq K .
\end{aligned}
$$

Since

$$
\begin{aligned}
\left|\tilde{z}_{\alpha l}(\alpha, t)\right|^{2} & =\frac{1}{l^{4}}\left|\int_{0}^{l}\left(z_{\alpha}(\alpha, t)-z_{\alpha}(\alpha+h, t)\right) d h\right|^{2} \\
& \leq \frac{1}{\mid l l^{5 / 2}} \int_{0}^{l}|h|^{-1 / 2}\left|z_{\alpha}(\alpha, t)-z_{\alpha}(\alpha+h, t)\right|^{2} d h \\
& =U_{[\alpha l]}(\alpha, t),
\end{aligned}
$$

and for the same reason

$$
\left|\tilde{z}_{t l}(\alpha, t)\right|^{2} \leq U_{[t l]}(\alpha, t)
$$

by a slightly modified discussion similar to that for theorem 3(ii) in Evans [24, sec. 5.8.2]; from (3.37) we conclude that

$$
z_{\alpha} \in H^{1}\left(\kappa_{1} I \times \kappa_{2}[0, T]\right) \quad \text { for any } 0<\kappa_{1}, \kappa_{2}<1 \text {; }
$$

moreover,

$$
\begin{aligned}
& \frac{1}{2} \int \delta(t) \int_{I} \operatorname{dist}^{2}(\alpha, \partial I)\left|z_{\alpha t}(\alpha, t)\right|^{2} d \alpha d t \\
& \quad+\frac{1}{16 M^{4}} \int \delta(t) \int_{I} \operatorname{dist}^{2}(\alpha, \partial I)\left|z_{\alpha \alpha}(\alpha, t)\right|^{2} d \alpha d t \leq K .
\end{aligned}
$$

Notice that $[c, d] \subset(a, b)$ is arbitrary, and $I \subset[c, d]$ is any interval with $|I| \leq$ $\frac{1}{2} \delta_{1}$. This proves the first part of Proposition 3.3.

\section{4 $H^{1 / 2}$ Compatibility of the Initial Data}

The second part of Proposition 3.3 can be proved by modifying the above argument. Taking $\zeta(t)=(T-t)^{2}$ for $t \in[0, T]$ and $\zeta(t)=0$ for $t \notin[0, T]$ as in the 
heuristic argument, and using integration by parts, we get from (3.29) that

$$
\begin{aligned}
& \frac{1}{2} \int \zeta(t) \int\left|\eta(\alpha) z_{t h}(\alpha, t)\right|^{2} d \alpha d t \\
& +\frac{1}{4 M^{4}} \int \zeta(t) \int\left|\eta(\alpha) z_{\alpha h}(\alpha, t)\right|^{2} d \alpha d t \\
& \leq \frac{T^{2}}{4 \pi} \operatorname{Im} \iint \frac{\eta(\alpha) \eta(\beta)\left(z_{h}(\alpha, 0)-z_{h}(\beta, 0)\right)^{2}}{(\alpha-\beta)^{2} z_{h}(\alpha, 0) z_{h}(\beta, 0)} d \alpha d \beta \\
& \quad+\frac{1}{4 \pi} \operatorname{Im} \int \zeta^{\prime}(t) \iint \frac{\eta(\alpha) \eta(\beta)\left(z_{h}(\alpha, t)-z_{h}(\beta, t)\right)^{2}}{(\alpha-\beta)^{2} z_{h}(\alpha, t) z_{h}(\beta, t)} d \alpha d \beta d t \\
& \quad+\frac{c_{3}}{|Q|} \int \zeta(t) d t \\
& \quad+\left(c_{1} \sup _{[0, T]}\left\|\ln z_{\alpha}\right\|_{*, \delta_{0}}^{2}+c_{2} \sup _{[0, T]}\left\|\ln z_{\alpha}\right\|_{*, \delta_{0}}^{1 / 2}\right) \\
& \quad \cdot \int \zeta(t) \int\left(\left|z_{\alpha h}(\alpha, t)\right|^{2}+\left|\tilde{z}_{\alpha h}(\alpha, t)\right|^{2}\right) d \alpha d t .
\end{aligned}
$$

Using the same argument as that from (3.30) leading to (3.37), with a similar consideration as that in the heuristic argument from (3.15) to (3.19) to deal with the extra term involving the initial data, defining $\theta(\alpha)$ as in (3.16), and taking into consideration the relations (3.17), (3.38), (3.39), and

$$
\begin{aligned}
2 \sum_{i, k}\left|I_{i, k}\right|^{2} \eta_{i, k}(\alpha) \eta_{i, k}(\beta)-2 \theta(\alpha) \theta(\beta) & \\
= & (\theta(\alpha)-\theta(\beta))^{2}-\sum_{i, k}\left|I_{i, k}\right|^{2}\left(\eta_{i, k}(\alpha)-\eta_{i, k}(\beta)\right)^{2},
\end{aligned}
$$

we conclude that for any closed interval $[c, d] \subset(a, b), I \subset[c, d]$, with $|I| \leq \frac{1}{2} \delta_{1}$, and for the same $c(m, M)$ as defined in (3.34), there is a constant $K$, independent of $l$, such that for $0<|l|<\frac{1}{2} \delta_{1}$,

$$
\begin{aligned}
& \frac{1}{2} \int \zeta(t) \int_{I}\left|\theta(\alpha) \tilde{z}_{t l}(\alpha, t)\right|^{2} d \alpha d t+\frac{1}{16 M^{4}} \int \zeta(t) \int_{I}\left|\theta(\alpha) \tilde{z}_{\alpha l}(\alpha, t)\right|^{2} d \alpha d t \\
& \quad \leq \frac{T^{2}}{4 \pi} \operatorname{Im} \frac{\operatorname{sign} l}{|l|^{5 / 2}} \int_{0}^{l}|h|^{3 / 2} \iint \frac{\theta(\alpha) \theta(\beta)\left(z_{h}(\alpha, 0)-z_{h}(\beta, 0)\right)^{2}}{(\alpha-\beta)^{2} z_{h}(\alpha, 0) z_{h}(\beta, 0)} d \alpha d \beta d h+K .
\end{aligned}
$$

Notice that the right-hand side of inequality (3.43) is fully nonlinear in $z_{\alpha}(\cdot, 0)$ and $\ln z_{\alpha}(\cdot, 0)$, while we have assumed only that the initial data satisfies

$$
\operatorname{Im}(1+i) \ln z_{\alpha}(\cdot, 0) \in H_{\text {loc }}^{1 / 2}(a, b) .
$$

We need to establish one further result that shows that the right-hand side of (3.43) is in fact almost bilinear in $\ln z_{\alpha}(\cdot, 0)$. This result is stated in Proposition 3.6. Once Proposition 3.6 is established, the second part of Proposition 3.3 will follow from (3.43). 
Take $\phi \in \mathfrak{S}(\mathbb{R})$ even such that its Fourier transform $\hat{\phi}(\xi)=1$ for $|\xi| \leq 1$ and $\hat{\phi}(\xi)=0$ for $|\xi|>2, \hat{\phi} \in C_{0}^{\infty}(\mathbb{R})$. Recall that we define $\phi^{h}(\alpha)=1 /|h| \bar{\phi}(\alpha / h)$. We have the following:

Proposition 3.6 Let $I \subset \mathbb{R}$ be an interval, $z: \mathbb{R} \rightarrow \mathbb{C}$ be a complex-valued function of period $4|I|$ satisfying

$$
m|\alpha-\beta| \leq|z(\alpha)-z(\beta)| \leq M|\alpha-\beta| \text { for all } \alpha, \beta \in \mathbb{R}
$$

for some constants $m, M>0$. Assume $\theta \in C^{0,1}(\mathbb{R})$, $\operatorname{supp} \theta \subset I$, and $\theta \geq 0$. Then for any $\epsilon>0$, there are constants $0<r<8, c_{0}>0$, and $K_{1}<\infty$, with $r$ and $c_{0}$ depending only on $m, M$, and $\epsilon$, such that if there is a determination of $\ln z_{\alpha}$ satisfying $\left\|\ln z_{\alpha}\right\|_{\mathrm{BMO}(4 I)} \leq c_{0}$, then for all $0<|l| \leq 1$,

$$
\begin{aligned}
& \left|\int_{0}^{l}\right| \iint \frac{\theta(\alpha) \theta(\beta)}{(\alpha-\beta)^{2}}\left(\frac{\left(z_{h}(\alpha)-z_{h}(\beta)\right)^{2}}{z_{h}(\alpha) z_{h}(\beta)}\right. \\
& \left.\quad-\left(\phi^{r h} *\left[\ln z_{\alpha}\right]_{h}(\alpha)-\phi^{r h} *\left[\ln z_{\beta}\right]_{h}(\beta)\right)^{2}\right) d \alpha d \beta|d h| \\
& \leq\left.\epsilon\left|\int_{0}^{l} \int\right||D|^{1 / 2}\left[\theta z_{\alpha}\right]_{h}(\alpha)\right|^{2} d \alpha d h \mid \\
& \quad+\left.\epsilon\left|\int_{0}^{l} \int\right||D|^{1 / 2} \phi^{r h} *\left[\theta \ln z_{\alpha}\right]_{h}(\alpha)\right|^{2} d \alpha d h\left|+K_{1}\right| l \mid .
\end{aligned}
$$

Remark 3.7. The constant $K_{1}$ depends on $\left\|\ln z_{\alpha}\right\|_{L^{2}(4 I)},\left\|\ln z_{\alpha}\right\|_{\mathrm{BMO}(4 I)}, m, M, \epsilon^{-1}$, $|I|,\left\|\theta^{\prime}\right\|_{L^{\infty}}$, and $\|\theta\|_{L^{\infty}}$, and is independent of $l ; K_{1}<\infty$ if all the aforementioned quantities are $<\infty$.

We also state the following:

LEMma 3.8 Let $I \subset \mathbb{R}$ be an interval, $\theta \in C^{0,1}(\mathbb{R})$, $\operatorname{supp} \theta \subset I$, and $\varphi: \mathbb{R} \rightarrow \mathbb{R}$ be a function such that $\varphi(x), \varphi^{\prime}(x), x \varphi(x)$, and $x \varphi^{\prime}(x) \in L^{1}(\mathbb{R})$. Let

$$
\begin{aligned}
& K_{2}= K_{2}(\theta, \varphi) \\
&=\left\|\theta^{\prime}\right\|_{L^{\infty}}\|\theta\|_{L^{\infty}}\left(\|\varphi\|_{L^{1}(\mathbb{R})}^{2}+\|x \varphi\|_{L^{1}(\mathbb{R})}\left\|\varphi^{\prime}\right\|_{L^{1}(\mathbb{R})}\right. \\
&\left.+\left\|x \varphi^{\prime}\right\|_{L^{1}(\mathbb{R})}\|\varphi\|_{L^{1}(\mathbb{R})}\right) .
\end{aligned}
$$

There exists a constant $c_{4}>0$, independent of $f, \varphi$, and $\theta$, such that

$$
\begin{aligned}
& \mid \iint \frac{\theta(\alpha) \theta(\beta)}{(\alpha-\beta)^{2}}(f * \varphi(\alpha)-f * \varphi(\beta))^{2} d \alpha d \beta \\
& \quad-\iint \frac{((\theta f) * \varphi(\alpha)-(\theta f) * \varphi(\beta))^{2}}{(\alpha-\beta)^{2}} d \alpha d \beta \mid \\
& \quad \leq c_{4} K_{2}\left(\|f\|_{L^{2}(4 I)}^{2}+|I|\|f\|_{\mathrm{BMO}(4 I)}\|f\|_{L^{2}(4 I)}\right),
\end{aligned}
$$

for all $f: \mathbb{R} \rightarrow \mathbb{R}$ periodic with period $4|I|$ and $f \in L^{2}(4 I) \cap \mathrm{BMO}(4 I)$.

The proof is straightforward and thus omitted. 
Remark 3.9. Notice that there is a constant $\bar{K}_{2}$, independent of $h$ and $r$, such that $K_{2}\left(\theta,\left[\phi^{r h}\right]_{h}\right) \leq \bar{K}_{2}$ for all $h \neq 0$ and $0<r<8$.

Remark 3.10. Lemma 3.8 also applies to $\varphi=\frac{1}{h} \chi_{[0, h]}$, with $K_{2}\left(\theta, \frac{1}{h} \chi_{[0, h]}\right) \leq \tilde{K}_{2}$, for some constant $\tilde{K}_{2}$ independent of $h \neq 0$.

Remark 3.11. The main point of Lemma 3.8 is that commutating with a smooth function gains one more order of derivative. We will use Lemma 3.8 and similar results in the same spirit as Lemma 3.8 a few times in the following. We refer to all of them as "Lemma 3.8."

Besides that in Section 2, we introduce one further notation that

$$
\tilde{[} f]_{h}=\frac{1}{h^{2}} \int_{0}^{h} \int_{\alpha}^{\alpha+r} f(s) d s d r .
$$

Assuming Proposition 3.6 holds, we prove the second part of Proposition 3.3. That is, we show that if we assume in addition that $\operatorname{Im}\left((1+i) \ln z_{\alpha}(\cdot, 0)\right) \in$ $H_{\mathrm{loc}}^{1 / 2}(a, b)$, then $\operatorname{Re}\left((1+i) \ln z_{\alpha}(\cdot, 0)\right) \in H_{\mathrm{loc}}^{1 / 2}(a, b)$ and $z_{\alpha} \in H^{1}(K)$ for any compact subset $K$ in $(a, b) \times[0, T)$.

Let $I \subset[c, d] \subset(a, b)$ be an arbitrary interval with $|I| \leq \frac{1}{2} \delta_{1}$. Notice that

$$
\begin{aligned}
T^{2} \int & \theta(\alpha) \overline{\tilde{z}_{l}(\alpha, 0)}|D|\left(\theta(\alpha) \tilde{z}_{l}(\alpha, 0)\right) d \alpha \\
= & -\int_{0}^{T} \int \partial_{t}\left(\zeta(t) \theta(\alpha) \overline{\tilde{z}_{l}(\alpha, t)}|D|\left(\theta(\alpha) \tilde{z}_{l}(\alpha, t)\right)\right) d \alpha d t \\
= & -\int_{0}^{T} \int \zeta^{\prime}(t) \theta(\alpha) \overline{\tilde{z}_{l}(\alpha, t)}|D|\left(\theta(\alpha) \tilde{z}_{l}(\alpha, t)\right) d \alpha d t \\
& \left.-2 \operatorname{Re} \int_{0}^{T} \int \zeta(t) \theta(\alpha) \overline{\tilde{z}_{t l}(\alpha, t)}|D|\left(\theta(\alpha) \tilde{z}_{l}(\alpha, t)\right)\right) d \alpha d t \\
\leq & \left.c_{5} \int_{0}^{T} \int \theta(\alpha) \tilde{z}_{l}(\alpha, t)\right|^{2} d \alpha d t+c_{5} \int_{0}^{T} \int \zeta(t)\left|\theta^{\prime}(\alpha) \tilde{z}_{l}(\alpha, t)\right|^{2} d \alpha d t \\
& +8 M^{2}\left(\frac{1}{2} \int \zeta(t) \int_{I}\left|\theta(\alpha) \tilde{z}_{t l}(\alpha, t)\right|^{2} d \alpha d t\right. \\
& \left.+\frac{1}{16 M^{4}} \int_{I} \zeta(t) \int_{I}\left|\theta(\alpha) \tilde{z}_{\alpha l}(\alpha, t)\right|^{2} d \alpha d t\right),
\end{aligned}
$$

where $c_{5}$ is a constant depending on $M$. 
Let $Z: \mathbb{R} \rightarrow \mathbb{C}$ be the reflective periodic extension of $z(\cdot, 0)$ from the interval $2 I$ to $\mathbb{R}$. Notice that for $\alpha \in I$ and $0<|h|<|I| / 2, z_{h}(\alpha, 0)=Z_{h}(\alpha)$. Let $0<$ $|\iota|<\min \{|I| / 2,1\}$. We apply Proposition 3.6 to $Z$. We have that for any $\epsilon>0$, there are $0<r<8$ and $c_{0}>0$ such that whenever $\left\|\ln z_{\alpha}(\cdot, 0)\right\|_{\mathrm{BMO}(2 I)} \leq c_{0}$,

$$
\begin{array}{r}
\text { Im sign } \iota \int_{0}^{\iota}\left(1-\left(\frac{h}{\iota}\right)^{3 / 2}\right) \\
\cdot \iint \frac{\theta(\alpha) \theta(\beta)}{(\alpha-\beta)^{2}} \frac{\left(z_{h}(\alpha, 0)-z_{h}(\beta, 0)\right)^{2}}{z_{h}(\alpha, 0) z_{h}(\beta, 0)} d \alpha d \beta d h \\
\leq \operatorname{Imsign~} \iota \int_{0}^{\iota}\left(1-\left(\frac{h}{\iota}\right)^{3 / 2}\right) \\
\cdot \iint \frac{\theta(\alpha) \theta(\beta)}{(\alpha-\beta)^{2}}\left(\phi^{r h} *\left[\ln z_{\alpha}\right]_{h}(\alpha)\right. \\
+\left.\epsilon\left|\int_{0}^{\iota} \int\right||D|^{1 / 2}\left[\theta z_{\alpha}(\cdot, 0)\right]_{h}\right|^{2} d \alpha d h \mid \\
+\left.\epsilon\left|\int_{0}^{\iota} \int\right||D|^{1 / 2} \phi^{r h} *\left[\theta \ln z_{\alpha}(\cdot, 0)\right]_{h}\right|^{2} d \alpha d h\left|+K_{1}\right| \iota \mid .
\end{array}
$$

Integrate (3.43) with respect to $l$ from 0 to $\iota$ if $\iota>0$ and from $\iota$ to 0 if $\iota<0$. From (3.47), (3.48), and Lemma 3.8, we get

$$
\begin{aligned}
& \left.\left|\int_{0}^{\iota} \int\right||D|^{1 / 2}\left[\theta z_{\alpha}(\cdot, 0)\right]_{h}\right|^{2} d \alpha d h \mid \\
& \quad \leq \operatorname{Im} \frac{8 M^{2}}{3} \operatorname{sign} \iota \int_{0}^{\iota}\left(1-\left(\frac{h}{\iota}\right)^{3 / 2}\right) \\
& \quad \cdot \int \phi^{r h} *\left[\theta \ln z_{\alpha}(\cdot, 0)\right]_{h}|D|\left(\phi^{r h} *\left[\theta \ln z_{\alpha}(\cdot, 0)\right]_{h}\right) d \alpha d h \\
& \quad+\left.\epsilon_{1}\left|\int_{0}^{\iota} \int\right||D|^{1 / 2}\left[\theta z_{\alpha}(\cdot, 0)\right]_{h}\right|^{2} d \alpha d h \mid \\
& \quad+\left.\epsilon_{1}\left|\int_{0}^{\iota} \int\right||D|^{1 / 2} \phi^{r h} *\left[\theta \ln z_{\alpha}(\cdot, 0)\right]_{h}\right|^{2} d \alpha d h\left|+K_{3}\right| \iota \mid
\end{aligned}
$$

where $\epsilon_{1}=\left(8 M^{2} / 3\right) \epsilon$ and $K_{3}$ is independent of $\iota$. ( $K_{3}$ depends on $M, m, T,|I|$, $\epsilon^{-1},\left\|\theta^{\prime}\right\|_{L^{\infty}},\|\theta\|_{L^{\infty}},\left\|\ln z_{\alpha}(\cdot, 0)\right\|_{L^{2}(2 I)},\left\|\ln z_{\alpha}(\cdot, 0)\right\|_{\mathrm{BMO}(2 I)}$, and the constant $K$ in (3.43).) Let $(1+i) \ln z_{\alpha}(\cdot, 0)=v+i w$, where $v=\operatorname{Re}\left\{(1+i) \ln z_{\alpha}(\cdot, 0)\right\}$ and 
$w=\operatorname{Im}\left\{(1+i) \ln z_{\alpha}(\cdot, 0)\right\}$. From (3.49) we get

$$
\begin{aligned}
& \left.\left|\int_{0}^{\iota} \int\right||D|^{1 / 2}\left[\theta z_{\alpha}(\cdot, 0)\right]_{h}\right|^{2} d \alpha d h \mid \\
& +\left.\frac{8 M^{2}}{3}\left|\int_{0}^{\iota}\left(1-\left(\frac{h}{\iota}\right)^{3 / 2}\right) \int\right||D|^{1 / 2} \phi^{r h} *\left[\theta \ln z_{\alpha}(\cdot, 0)\right]_{h}\right|^{2} d \alpha d h \mid \\
& \leq\left.\frac{8 M^{2}}{3}\left|\int_{0}^{\iota}\left(1-\left(\frac{h}{\iota}\right)^{3 / 2}\right) \int\right||D|^{1 / 2} \phi^{r h} *[\theta w]_{h}\right|^{2} d \alpha d h \mid \\
& +\left.\epsilon_{1}\left|\int_{0}^{\iota} \int\right||D|^{1 / 2}\left[\theta z_{\alpha}(\cdot, 0)\right]_{h}\right|^{2} d \alpha d h \mid \\
& +\left.\epsilon_{1}\left|\int_{0}^{\iota} \int\right||D|^{1 / 2} \phi^{r h} *\left[\theta \ln z_{\alpha}(\cdot, 0)\right]_{h}\right|^{2} d \alpha d h\left|+K_{3}\right| \iota \mid .
\end{aligned}
$$

Notice that

$$
\begin{array}{rl}
\left.\left|\int_{0}^{\iota}\left(1-\left(\frac{h}{\iota}\right)^{3 / 2}\right) \int\right||D|^{1 / 2} \phi^{r h} *[\theta w]_{h}\right|^{2} & d \alpha d h \mid \\
& \leq\left.\left. C \iota \int|| D\right|^{1 / 2}(\theta w)\right|^{2} d \alpha<\infty
\end{array}
$$

for some constant $C$ independent of $\iota$. Using Fourier analysis and Plancherel's theorem on both sides of (3.50) and taking a small enough $\epsilon_{1} \neq 0\left(\epsilon_{1}\right.$ depends on $M$ only), we conclude that there are $0<r<8$ and $c_{0}>0, r$ and $c_{0}$ depending on $m$ and $M$ only, such that if

$$
\sup _{[0, T]}\left\|\ln z_{\alpha}(\cdot, t)\right\|_{\mathrm{BMO}(a, b), \delta_{0}} \leq \min \left\{c_{0}, c(m, M)\right\}
$$

with $c(m, M)$ as in (3.34), then

$$
\begin{array}{r}
\left.\left.\frac{1}{\iota} \int_{0}^{\iota} \int|| D\right|^{1 / 2}\left[\theta z_{\alpha}(\cdot, 0)\right]_{h}\right|^{2} d \alpha d h \leq K_{4}, \\
\left.\left.\frac{1}{\iota} \int_{0}^{\iota} \int|| D\right|^{1 / 2} \phi^{r h} *\left[\theta \ln z_{\alpha}(\cdot, 0)\right]_{h}\right|^{2} d \alpha d h \leq K_{4},
\end{array}
$$

for some constant $K_{4}$ depending on $K_{3}, M$, and $\left.\left.\int|| D\right|^{1 / 2}(\theta w)\right|^{2} d \alpha$ only.

Let $\iota \rightarrow 0$. Since $I \subset[c, d] \subset(a, b)$ and $|I| \leq \frac{1}{2} \delta_{1}$ is arbitrary, we get $z_{\alpha}(\cdot, 0), \ln z_{\alpha}(\cdot, 0) \in H_{\text {loc }}^{1 / 2}(a, b)$. The fact that $z_{\alpha} \in H^{1}(K)$ for $K$ compact in $(a, b) \times[0, T)$ therefore follows directly from (3.43) by taking $l \rightarrow 0$.

We now prove Proposition 3.6. Assume $z, \theta$, and $\phi$ satisfy the assumption of Proposition 3.6. We proceed by decomposing the term

$$
\begin{aligned}
& \iint \frac{\theta(\alpha) \theta(\beta)}{(\alpha-\beta)^{2}} \\
& \quad \cdot\left(\frac{\left(z_{h}(\alpha)-z_{h}(\beta)\right)^{2}}{z_{h}(\alpha) z_{h}(\beta)}-\left(\phi^{8 r h} *\left[\ln z_{\alpha}\right]_{h}(\alpha)-\phi^{8 r h} *\left[\ln z_{\beta}\right]_{h}(\beta)\right)^{2}\right) d \alpha d \beta
\end{aligned}
$$


into several terms and then proving each of them to be relatively small compared with $\left.\left.\int|| D\right|^{1 / 2}\left[\theta z_{\alpha}\right]\right|^{2} d \alpha$ and $\left.\left.\int|| D\right|^{1 / 2}\left[\theta \ln z_{\alpha}\right]\right|^{2} d \alpha$ for suitably chosen $0<r<1$ and small enough $\left\|\ln z_{\alpha}\right\|_{\mathrm{BMO}(4 I)}$. Notice that for convenience in writing the proof, we have replaced $\phi^{r h}$ in (3.44) by $\phi^{8 r h}$. To validate the decomposition, we need the following lemma.

Lemma 3.12 Assume that $z$ and $\phi$ satisfy the assumption of Proposition 3.6. Then for any $|h|>0, r>0$, and $\alpha \in \mathbb{R}$,

(i) $\left|z_{h} * \phi^{r h}(\alpha)-z_{h}(\alpha)\right| \leq 2 r M\|x \phi\|_{L^{1}(\mathbb{R})}$,

(ii) $\left|z_{\alpha} * \phi^{h}(\alpha)-z_{h}(\alpha)\right| \leq \sum_{n=-\infty}^{\infty} 2 n^{2} \max _{[n-1, n]}|\phi(x)|\left\|z_{\alpha}\right\|_{\mathrm{BMO}(\mathbb{R})}$.

PROOF:

(i) Notice that $\int \phi=1$. And for any $\alpha, \beta$, and $h \neq 0,\left|z_{h}(\alpha)-z_{h}(\beta)\right| \leq$ $2 M|\alpha-\beta| /|h|$. We have for any $\alpha \in \mathbb{R}, r>0$, and $h \neq 0$,

$$
\begin{aligned}
\left|z_{h} * \phi^{r h}(\alpha)-z_{h}(\alpha)\right| & =\left|\int\left(z_{h}(\alpha)-z_{h}(\beta)\right) \phi^{r h}(\alpha-\beta) d \beta\right| \\
& \leq \int \frac{2 M|\alpha-\beta|}{|h|}\left|\phi^{r h}(\alpha-\beta)\right| d \beta=2 r M \int|x||\phi(x)| d x .
\end{aligned}
$$

(ii) For $h \neq 0$, we have for any $\alpha \in \mathbb{R}$,

$$
\begin{aligned}
\mid z_{\alpha} & * \phi^{h}(\alpha)-z_{h}(\alpha) \mid \\
& \leq \frac{1}{h} \int_{0}^{h} \int\left|z_{\alpha}(\alpha+\gamma)-z_{\alpha}(\alpha+\beta)\right| \phi^{h}(\beta) \mid d \beta d \gamma \\
& =\sum_{n=-\infty}^{\infty} \frac{1}{h^{2}} \int_{0}^{h} \int_{(n-1) h}^{n h}\left|z_{\alpha}(\alpha+\gamma)-z_{\alpha}(\alpha+\beta)\right|\left|\phi\left(\frac{\beta}{h}\right)\right| d \beta d \gamma \\
& \leq \sum_{n=-\infty}^{\infty} \frac{\max _{[n-1, n]}|\phi(x)|}{h^{2}} \int_{0}^{h} \int_{(n-1) h}^{n h}\left|z_{\alpha}(\alpha+\gamma)-z_{\alpha}(\alpha+\beta)\right| d \beta d \gamma,
\end{aligned}
$$

while

$$
\begin{aligned}
& \frac{1}{h^{2}} \int_{0}^{h} \int_{(n-1) h}^{n h}\left|z_{\alpha}(\alpha+\beta)-z_{\alpha}(\alpha+\gamma)\right| d \beta d \gamma \\
& \quad \leq \frac{n^{2}}{n^{2} h^{2}} \int_{\alpha}^{\alpha+n h} \int_{\alpha}^{\alpha+n h}\left|z_{\alpha}(\beta)-z_{\alpha}(\gamma)\right| d \beta d \gamma \\
& \quad \leq \frac{2 n^{2}}{n h} \int_{\alpha}^{\alpha+n h}\left|z_{\alpha}(\beta)-m_{[\alpha, \alpha+n h]}\left(z_{\alpha}\right)\right| d \beta \leq 2 n^{2}\left\|z_{\alpha}\right\|_{\mathrm{BMO}(\mathbb{R})} .
\end{aligned}
$$

Assertion (ii) therefore follows.

It follows from Lemma 3.12 that for

$$
\left\|z_{\alpha}\right\|_{\mathrm{BMO}(\mathbb{R})} \leq \frac{m}{4 \sum_{-\infty}^{\infty} n^{2} \max _{[n-1, n]}|\phi(x)|} \quad \text { and } \quad 0<r \leq \frac{m}{4 M\|x \phi\|_{L^{1}(\mathbb{R})}},
$$


we have

$$
\left|z_{h} * \phi^{r h}(\alpha)\right| \geq \frac{m}{2}, \quad\left|z_{\alpha} * \phi^{h}(\alpha)\right| \geq \frac{m}{2}, \quad \text { for all } \alpha \in \mathbb{R}, h \neq 0 .
$$

We know from Propositions 2.7 and 2.8 that if $\left\|\ln z_{\alpha}\right\|_{\mathrm{BMO}(4 I)} \leq r_{1} / 8$, where $r_{1}$ is the constant in Proposition 2.7, then

$$
\left\|z_{\alpha}\right\|_{\mathrm{BMO}(\mathbb{R})} \leq C_{1}\left\|\ln z_{\alpha}\right\|_{\mathrm{BMO}(\mathbb{R})} \leq 8 C_{1}\left\|\ln z_{\alpha}\right\|_{\mathrm{BMO}(4 I)}
$$

for some constant $C_{1}$. We begin with

$$
\begin{aligned}
0<r \leq & \frac{m}{4 M\|x \phi\|_{L^{1}(\mathbb{R})}}, \\
& \left\|\ln z_{\alpha}\right\|_{\mathrm{BMO}(4 I)} \leq \min \left\{\frac{r_{1}}{8}, \frac{m}{32 C_{1} \sum_{-\infty}^{\infty} n^{2} \max _{[n-1, n]}|\phi(x)|}\right\},
\end{aligned}
$$

where $r_{1}$ and $C_{1}$ are the constants in Proposition 2.7. Now

$$
\begin{aligned}
& \iint \frac{\theta(\alpha) \theta(\beta)}{(\alpha-\beta)^{2}}\left(\frac{\left(z_{h}(\alpha)-z_{h}(\beta)\right)^{2}}{z_{h}(\alpha) z_{h}(\beta)}\right. \\
& \left.\quad-\left(\phi^{8 r h} *\left[\ln z_{\alpha}\right]_{h}(\alpha)-\phi^{8 r h} *\left[\ln z_{\beta}\right]_{h}(\beta)\right)^{2}\right) d \alpha d \beta \\
& =\iint \frac{\theta(\alpha) \theta(\beta)}{(\alpha-\beta)^{2}}\left(\frac{z_{h}(\alpha)}{z_{h}(\beta)}+\frac{z_{h}(\beta)}{z_{h}(\alpha)}-\frac{z_{h} * \phi^{r h}(\alpha)}{z_{h} * \phi^{r h}(\beta)}-\frac{z_{h} * \phi^{r h}(\beta)}{z_{h} * \phi^{r h}(\alpha)}\right) d \alpha d \beta \\
& +\iint \frac{\theta(\alpha) \theta(\beta)}{(\alpha-\beta)^{2}}\left(\frac{z_{h} * \phi^{r h}(\alpha)}{z_{h} * \phi^{r h}(\beta)}+\frac{z_{h} * \phi^{r h}(\beta)}{z_{h} * \phi^{r h}(\alpha)}\right. \\
& \left.\quad-\frac{e^{\phi^{8 r h} *\left[\ln z_{\alpha}\right]_{h}(\alpha)}}{e^{8 r h} *\left[\ln z_{\beta}\right]_{h}(\beta)}-\frac{e^{\phi^{8 r h} *\left[\ln z_{\beta}\right]_{h}(\beta)}}{e^{8 r h} *\left[\ln z_{\alpha}\right]_{h}(\alpha)}\right) d \alpha d \beta \\
& +\iint \frac{\theta(\alpha) \theta(\beta)}{(\alpha-\beta)^{2}}\left(\frac{e^{\phi^{8 r h} *\left[\ln z_{\alpha}\right]_{h}(\alpha)}}{e^{\phi^{8 r h} *\left[\ln z_{\beta}\right]_{h}(\beta)}}-\frac{e^{\phi^{8 r h} *\left[\ln z_{\beta}\right]_{h}(\beta)}}{e^{\phi^{8 r h} *\left[\ln z_{\alpha}\right]_{h}(\alpha)}}-2\right. \\
& \left.\quad-\left(\phi^{8 r h} *\left[\ln z_{\alpha}\right]_{h}(\alpha)-\phi^{8 r h} *\left[\ln z_{\beta}\right]_{h}(\beta)\right)^{2}\right) d \alpha d \beta \\
& =\mathrm{I}_{1}+\mathrm{I}_{2}+\mathrm{I}_{3} . \quad
\end{aligned}
$$

where

$$
\begin{gathered}
\mathrm{I}_{1}=\iint \frac{\theta(\alpha) \theta(\beta)}{(\alpha-\beta)^{2}}\left(\frac{z_{h}(\alpha)}{z_{h}(\beta)}+\frac{z_{h}(\beta)}{z_{h}(\alpha)}-\frac{z_{h} * \phi^{r h}(\alpha)}{z_{h} * \phi^{r h}(\beta)}-\frac{z_{h} * \phi^{r h}(\beta)}{z_{h} * \phi^{r h}(\alpha)}\right) d \alpha d \beta, \\
\mathrm{I}_{2}=\iint \frac{\theta(\alpha) \theta(\beta)}{(\alpha-\beta)^{2}}\left(\frac{z_{h} * \phi^{r h}(\alpha)}{z_{h} * \phi^{r h}(\beta)}+\frac{z_{h} * \phi^{r h}(\beta)}{z_{h} * \phi^{r h}(\alpha)}\right. \\
\left.-\frac{e^{\phi^{8 r h} *\left[\ln z_{\alpha}\right]_{h}(\alpha)}}{e^{\phi^{8 r h} *\left[\ln z_{\beta}\right]_{h}(\beta)}}-\frac{e^{\phi^{8 r h} *\left[\ln z_{\beta}\right]_{h}(\beta)}}{e^{\phi^{8 r h} *\left[\ln z_{\alpha}\right]_{h}(\alpha)}}\right) d \alpha d \beta,
\end{gathered}
$$




$$
\begin{aligned}
\mathrm{I}_{3}=\iint \frac{\theta(\alpha) \theta(\beta)}{(\alpha-\beta)^{2}}\left(\frac{e^{\phi^{8 r h} *\left[\ln z_{\alpha}\right]_{h}(\alpha)}}{e^{\phi^{8 r h} *\left[\ln z_{\beta}\right]_{h}(\beta)}}-\frac{e^{\phi^{8 r h} *\left[\ln z_{\beta}\right]_{h}(\beta)}}{e^{\phi^{8 r h} *\left[\ln z_{\alpha}\right]_{h}(\alpha)}}-2\right. \\
\left.\quad-\left(\phi^{8 r h} *\left[\ln z_{\alpha}\right]_{h}(\alpha)-\phi^{8 r h} *\left[\ln z_{\beta}\right]_{h}(\beta)\right)^{2}\right) d \alpha d \beta .
\end{aligned}
$$

For $\mathrm{I}_{1}$, we apply the identity that for any $u, v, u_{1}, v_{1} \neq 0$,

$$
\begin{aligned}
\frac{u}{v}+\frac{v}{u}-\frac{u_{1}}{v_{1}}-\frac{v_{1}}{u_{1}}= & -\frac{\left(u-u_{1}-v+v_{1}\right)^{2}}{u_{1} v_{1}}+\frac{(u-v)^{2}}{u v}\left(1-\frac{u v}{u_{1} v_{1}}\right) \\
& +2\left(u-u_{1}-v+v_{1}\right) \frac{(u-v)}{u_{1} v_{1}} .
\end{aligned}
$$

We get

$$
\begin{aligned}
\left|\mathrm{I}_{1}\right| \leq & \iint \frac{\theta(\alpha) \theta(\beta)}{(\alpha-\beta)^{2}} \frac{\left|z_{h}(\alpha)-z_{h} * \phi^{r h}(\alpha)-z_{h}(\beta)+z_{h} * \phi^{r h}(\beta)\right|^{2}}{\left|z_{h} * \phi^{r h}(\alpha) z_{h} * \phi^{r h}(\beta)\right|} d \alpha d \beta \\
& +\iint \frac{\theta(\alpha) \theta(\beta)}{(\alpha-\beta)^{2}} \frac{\left|z_{h}(\alpha)-z_{h}(\beta)\right|^{2}}{\left|z_{h}(\alpha) z_{h}(\beta)\right|}\left|1-\frac{z_{h}(\alpha) z_{h}(\beta)}{z_{h} * \phi^{r h}(\alpha) z_{h} * \phi^{r h}(\beta)}\right| d \alpha d \beta \\
& +2 \iint \frac{\theta(\alpha) \theta(\beta)}{(\alpha-\beta)^{2}} \frac{\left|z_{h}(\alpha)-z_{h} * \phi^{r h}(\alpha)-z_{h}(\beta)+z_{h} * \phi^{r h}(\beta)\right|\left|z_{h}(\alpha)-z_{h}(\beta)\right|}{\left|z_{h} * \phi^{r h}(\alpha) z_{h} * \phi^{r h}(\beta)\right|} d \alpha d \beta \\
\leq & \frac{4}{m^{2}}\left(1+r^{-1 / 2}\right) \iint \frac{\theta(\alpha) \theta(\beta)}{(\alpha-\beta)^{2}}\left|z_{h}(\alpha)-z_{h} * \phi^{r h}(\alpha)-z_{h}(\beta)+z_{h} * \phi^{r h}(\beta)\right|^{2} d \alpha d \beta \\
& +\left(\frac{16 M^{2}\|x \phi\|_{L^{1}(\mathbb{R})}}{m^{4}}+\frac{4}{m^{2}} r^{1 / 2}\right) \iint \frac{\theta(\alpha) \theta(\beta)}{(\alpha-\beta)^{2}}\left|z_{h}(\alpha)-z_{h}(\beta)\right|^{2} d \alpha d \beta .
\end{aligned}
$$

Here we used Lemma 3.12(i) and (3.52) in the above estimate. An application of Fourier analysis, Plancherel's theorem, and Lemma 3.8 gives

$$
\left|\int_{0}^{l}\right| I_{1}|d h| \leq\left. c_{1}\left(r+r^{1 / 2}\right)\left|\int_{0}^{l} \int\right||D|^{1 / 2}\left[\theta z_{\alpha}\right]_{h}(\alpha)\right|^{2} d \alpha d h\left|+K_{5}\right| l \mid
$$

where $c_{1}$ is a constant depending on $m$ and $M$, and $K_{5}$ is a constant depending on $m, M,|I|, r^{-1},\|\theta\|_{L^{\infty}}$, and $\left\|\theta^{\prime}\right\|_{L^{\infty}}$.

For $\mathrm{I}_{2}$, we use the identity that for any $u, v, u_{1}, v_{1} \neq 0$,

$$
\frac{u}{v}+\frac{v}{u}-\frac{u_{1}}{v_{1}}-\frac{v_{1}}{u_{1}}=\left(\frac{u}{u_{1}}-\frac{v}{v_{1}}\right) \frac{\left(u_{1}-v_{1}\right) u+v_{1}(u-v)}{u v} .
$$

Since the real part of $\ln z_{\alpha}$ is bounded and (3.52) holds, we have

$$
\begin{aligned}
& \mid \frac{z_{h} * \phi^{r h}(\alpha)}{z_{h} * \phi^{r h}(\beta)}+\frac{z_{h} * \phi^{r h}(\beta)}{z_{h} * \phi^{r h}(\alpha)}-\frac{e^{\phi^{8 r h} *\left[\ln z_{\alpha}\right]_{h}(\alpha)}}{e^{\phi^{8 r h} *\left[\ln z_{\beta}\right]_{h}(\beta)}-\frac{e^{\phi^{8 r h} *\left[\ln z_{\beta}\right]_{h}(\beta)}}{e^{\phi^{8 r h} *\left[\ln z_{\alpha}\right]_{h}(\alpha)}} \mid} \\
& \leq C\left|\frac{z_{h} * \phi^{r h}(\alpha)}{e^{\phi^{8 r h} *\left[\ln z_{\alpha}\right]_{h}(\alpha)}}-\frac{z_{h} * \phi^{r h}(\beta)}{e^{\phi^{8 r h} *\left[\ln z_{\beta}\right]_{h}(\beta)}}\right| \\
& \cdot\left(\left|z_{h} * \phi^{r h}(\alpha)-z_{h} * \phi^{r h}(\beta)\right|+\left|\phi^{8 r h} *\left[\ln z_{\alpha}\right]_{h}(\alpha)-\phi^{8 r h} *\left[\ln z_{\beta}\right]_{h}(\beta)\right|\right),
\end{aligned}
$$


where $C$ is a constant depending on $m$ and $M$. Let

$$
\mathrm{II}=\iint \frac{\theta(\alpha) \theta(\beta)}{(\alpha-\beta)^{2}}\left|\frac{z_{h} * \phi^{r h}(\alpha)}{e^{\phi^{8 r h} *\left[\ln z_{\alpha}\right]_{h}(\alpha)}}-\frac{z_{h} * \phi^{r h}(\beta)}{e^{\phi^{8 r h} *\left[\ln z_{\beta}\right]_{h}(\beta)}}\right|^{2} d \alpha d \beta .
$$

Therefore

$$
\begin{aligned}
\left|I_{2}\right| \leq C(\mathrm{II})^{1 / 2}\left\{\left(\iint \frac{\theta(\alpha) \theta(\beta)}{(\alpha-\beta)^{2}}\left|z_{h} * \phi^{r h}(\alpha)-z_{h} * \phi^{r h}(\beta)\right|^{2} d \alpha d \beta\right)^{1 / 2}\right. \\
+\iiint \frac{\theta(\alpha) \theta(\beta)}{(\alpha-\beta)^{2}} \mid \phi^{8 r h} *\left[\ln z_{\alpha}\right]_{h}(\alpha) \\
\left.\left.\quad-\left.\phi^{8 r h} *\left[\ln z_{\beta}\right]_{h}(\beta)\right|^{2} d \alpha d \beta\right)^{1 / 2}\right\} \\
\leq r^{-1 / 2} \mathrm{II}+C^{2} r^{1 / 2} \iint \frac{\theta(\alpha) \theta(\beta)}{(\alpha-\beta)^{2}}\left|z_{h} * \phi^{r h}(\alpha)-z_{h} * \phi^{r h}(\beta)\right|^{2} d \alpha d \beta \\
+C^{2} r^{1 / 2} \iint \frac{\theta(\alpha) \theta(\beta)}{(\alpha-\beta)^{2}} \mid \phi^{8 r h} *\left[\ln z_{\alpha}\right]_{h}(\alpha) \\
-\left.\phi^{8 r h} *\left[\ln z_{\beta}\right]_{h}(\beta)\right|^{2} d \alpha d \beta .
\end{aligned}
$$

From Lemma 3.8, we know

$$
\begin{aligned}
\iint \frac{\theta(\alpha) \theta(\beta)}{(\alpha-\beta)^{2}}\left|z_{h} * \phi^{r h}(\alpha)-z_{h} * \phi^{r h}(\beta)\right|^{2} d \alpha d \beta & \\
& =\left.\left.2 \pi \int|| D\right|^{1 / 2}\left[\theta z_{\alpha}\right]_{h} * \phi^{r h}\right|^{2} d \alpha+K_{6},
\end{aligned}
$$

where $K_{6}$ is a constant depending on $m, M,|I|,\|\theta\|_{L^{\infty}},\left\|\theta^{\prime}\right\|_{L^{\infty}}$, and

$$
\begin{array}{r}
\iint \frac{\theta(\alpha) \theta(\beta)}{(\alpha-\beta)^{2}}\left|\phi^{8 r h} *\left[\ln z_{\alpha}\right]_{h}(\alpha)-\phi^{8 r h} *\left[\ln z_{\beta}\right]_{h}(\beta)\right|^{2} d \alpha d \beta \\
=\left.\left.2 \pi \int|| D\right|^{1 / 2}\left[\theta \ln z_{\alpha}\right]_{h} * \phi^{8 r h}\right|^{2} d \alpha+K_{7},
\end{array}
$$

where $K_{7}$ depends on $\left\|\ln z_{\alpha}\right\|_{\mathrm{BMO}(4 I)},\left\|\ln z_{\alpha}\right\|_{L^{2}(4 I)}, m, M,|I|,\|\theta\|_{L^{\infty}}$, and $\left\|\theta^{\prime}\right\|_{L^{\infty}}$. 
We now consider II. We know from the assumption of Proposition 3.6 and (3.52) that the real parts of $\ln z_{\alpha}$ and $\ln z_{\alpha} * \phi^{r h}$ are bounded. Therefore

$$
\begin{aligned}
&\left|\frac{z_{h} *}{e^{\phi^{8 r h}} *\left[\ln z_{\alpha}\right]_{h}(\alpha)}-\frac{z_{h} * \phi^{r h}(\beta)}{e^{\phi^{8 r h} *\left[\ln z_{\beta}\right]_{h}(\beta)}}\right| \\
& \leq c_{1}\left|\frac{z_{h} * \phi^{r h}(\alpha)}{e^{\left[\ln z_{\alpha} * \phi^{r h}\right]_{h}(\alpha)}}-\frac{z_{h} * \phi^{r h}(\beta)}{e^{\left[\ln z_{\beta} * \phi^{r h}\right] h}(\beta)}\right| \\
&+c_{1}\left|e^{\phi^{8 r h} *\left[\ln z_{\alpha} * \phi^{r h}-\ln z_{\alpha}\right]_{h}(\alpha)}-e^{\phi^{8 r h} *\left[\ln z_{\beta} * \phi^{r h}-\ln z_{\beta}\right]_{h}(\beta)}\right| \\
&+c_{1}\left|e^{\left[\ln z_{\alpha} * \phi^{r h}\right]_{h}(\alpha)-\phi^{8 r h} *\left[\ln z_{\alpha} * \phi^{r h}\right]_{h}(\alpha)}-e^{\left[\ln z_{\beta} * \phi^{r h}\right]_{h}(\beta)-\phi^{8 r h} *\left[\ln z_{\beta} * \phi^{r h}\right]_{h}(\beta)}\right| \\
& \leq c_{1} \mid \frac{z_{h} * \phi^{r h}(\alpha)}{e^{\left[\ln z_{\alpha} * \phi^{r h}\right]_{h}(\alpha)}}-\frac{z_{h} * \phi^{r h}(\beta)}{e^{\left[\ln z_{\beta} * \phi^{r h}\right]_{h}(\beta)} \mid} \\
&+c_{2}\left|\phi^{8 r h} *\left[\ln z_{\alpha} * \phi^{r h}-\ln z_{\alpha}\right]_{h}(\alpha)-\phi^{8 r h} *\left[\ln z_{\beta} * \phi^{r h}-\ln z_{\beta}\right]_{h}(\beta)\right| \\
&+c_{2} \mid\left[\ln z_{\alpha} * \phi^{r h}\right]_{h}(\alpha)-\phi^{8 r h} *\left[\ln z_{\alpha} * \phi^{r h}\right]_{h}(\alpha) \\
&\left.-\left[\ln z_{\beta} * \phi^{r h}\right]_{h}(\beta)+\phi^{8 r h} *\left[\ln z_{\beta} * \phi^{r h}\right]_{h}\right)(\beta) \mid,
\end{aligned}
$$

where $c_{1}$ and $c_{2}$ are constants depending on $m$ and $M$. Consequently,

$$
\mathrm{II} \leq 4 c_{1}^{2} \mathrm{II}_{1}+4 c_{2}^{2} \mathrm{II}_{2}+2 c_{2}^{2} \mathrm{II}_{3}
$$

where

$$
\begin{aligned}
& \mathrm{II}_{1}= \iint \frac{\theta(\alpha) \theta(\beta)}{(\alpha-\beta)^{2}}\left|\frac{z_{h} * \phi^{r h}(\alpha)}{e^{\left[\ln z_{\alpha} * \phi^{r h}\right]_{h}(\alpha)}}-\frac{z_{h} * \phi^{r h}(\beta)}{e^{\left[\ln z_{\beta} * \phi^{r h}\right]_{h}(\beta)}}\right|^{2} d \alpha d \beta, \\
& \mathrm{II}_{2}=\iint \frac{\theta(\alpha) \theta(\beta)}{(\alpha-\beta)^{2}} \mid \phi^{8 r h} *\left[\ln z_{\alpha} * \phi^{r h}-\ln z_{\alpha}\right]_{h}(\alpha) \\
& \quad-\left.\phi^{8 r h} *\left[\ln z_{\beta} * \phi^{r h}-\ln z_{\beta}\right]_{h}(\beta)\right|^{2} d \alpha d \beta, \\
& \mathrm{II}_{3}=\iint \frac{\theta(\alpha) \theta(\beta)}{(\alpha-\beta)^{2}} \mid\left[\ln z_{\alpha} * \phi^{r h}\right]_{h}(\alpha)-\phi^{8 r h} *\left[\ln z_{\alpha} * \phi^{r h}\right]_{h}(\alpha) \\
&\left.\quad-\left[\ln z_{\beta} * \phi^{r h}\right]_{h}(\beta)+\phi^{8 r h} *\left[\ln z_{\beta} * \phi^{r h}\right]_{h}\right)\left.(\beta)\right|^{2} d \alpha d \beta .
\end{aligned}
$$

We consider $\mathrm{II}_{3}$ first. Let $\psi_{1}$ be such that its Fourier transform is

$$
\hat{\psi}_{1}(\xi)=\frac{1-\hat{\phi}(\xi)}{\xi} \text {. }
$$

We know $\psi_{1}, \alpha \psi_{1}(\alpha), \alpha \psi_{1}^{\prime}(\alpha) \in L^{1}(\mathbb{R})$. Now

$$
\left[\ln z_{\alpha} * \phi^{r h}\right]_{h}(\alpha)-\phi^{8 r h} *\left[\ln z_{\alpha} * \phi^{r h}\right]_{h}(\alpha)=8 \psi_{1}^{8 r h} *\left[\frac{z_{\alpha} * \psi_{2}^{r h}}{z_{\alpha} * \phi^{r h}}\right]_{h}(\alpha),
$$


where

$$
\hat{\psi}_{2}(\xi)=\xi \hat{\phi}(\xi)
$$

Therefore

$$
\begin{aligned}
\mathrm{II}_{3}= & 64 \int \frac{\theta(\alpha) \theta(\beta)}{(\alpha-\beta)^{2}} \mid \psi_{1}^{8 r h} *\left[\frac{z_{\alpha} * \psi_{2}^{r h}}{z_{\alpha} * \phi^{r h}}\right]_{h}(\alpha) \\
& -\left.\psi_{1}^{8 r h} *\left[\frac{z_{\beta} * \psi_{2}^{r h}}{z_{\beta} * \phi^{r h}}\right]_{h}(\beta)\right|^{2} d \alpha d \beta \\
= & 64 \iint \frac{1}{(\alpha-\beta)^{2}} \mid \psi_{1}^{8 r h} *\left[\theta \frac{z_{\alpha} * \psi_{2}^{r h}}{z_{\alpha} * \phi^{r h}}\right]_{h}(\alpha) \\
& -\left.\psi_{1}^{8 r h} *\left[\theta \frac{z_{\beta} * \psi_{2}^{r h}}{z_{\beta} * \phi^{r h}}\right]_{h}(\beta)\right|^{2} d \alpha d \beta+K_{8} \\
& =c_{1} \int|\xi|\left|\frac{e^{i h \xi}-1}{h \xi}\right|^{2}\left|\frac{1-\hat{\phi}(8 r h \xi)}{8 r h \xi}\right|^{2}\left|\theta \frac{z_{\alpha} * \psi_{2}^{r h}}{z_{\alpha} * \phi^{r h}}(\xi)\right|^{2} d \xi+K_{8} \\
& \leq c_{2} \frac{r}{h} \int\left|\theta(\alpha) \frac{z_{\alpha} * \psi_{2}^{r h}}{z_{\alpha} * \phi^{r h}}(\alpha)\right|^{2} d \alpha+K_{8} \\
& \leq c_{3} \frac{r}{h} \int\left|\left(\theta z_{\alpha}\right) * \psi_{2}^{r h}\right|^{2} d \alpha+\bar{K}_{8},
\end{aligned}
$$

where $c_{1}$ and $c_{2}$ are universal constants, $c_{3}$ depends on $m$ and $M$, and $K_{8}$ and $\bar{K}_{8}$ depend on $m, M,|I|,\|\theta\|_{L^{\infty}}$, and $\left\|\theta^{\prime}\right\|_{L^{\infty}}$. In (3.64) we used Plancherel's theorem, Lemma 3.8, and the fact that $\hat{\phi}(\xi)=1$ for $|\xi| \leq 1$.

Applying Plancherel's theorem again, we have that for $0 \leq r \leq 1$,

$$
\left.\left.\left.\left|\int_{0}^{l} \int \frac{1}{h}\right|\left(\theta z_{\alpha}\right) * \psi_{2}^{r h}\right|^{2} d \alpha d h\left|\leq c_{4}\right| \int_{0}^{l} \int|| D\right|^{1 / 2}\left[\theta z_{\alpha}\right]_{h}\right|^{2} d \alpha d h \mid,
$$

where $c_{4}$ is a universal constant. Therefore

$$
r^{-1 / 2}\left|\int_{0}^{l} \mathrm{II}_{3} d h\right| \leq\left. c_{3} c_{4} r^{1 / 2}\left|\int_{0}^{l} \int\right||D|^{1 / 2}\left[\theta z_{\alpha}\right]_{h}\right|^{2} d \alpha d h\left|+\bar{K}_{8}\right| l \mid .
$$

Summing up the calculation from (3.57) through (3.65), we have

$$
\begin{aligned}
\left|\int_{0}^{l}\right| I_{2}|d h| \leq & \bar{c}_{1} r^{-1 / 2}\left|\int_{0}^{l} \mathrm{II}_{1} d h\right|+\bar{c}_{2} r^{-1 / 2}\left|\int_{0}^{l} \mathrm{II}_{2} d h\right| \\
& +\left.\bar{c}_{3} r^{1 / 2}\left|\int_{0}^{l} \int\right||D|^{1 / 2}\left[\theta z_{\alpha}\right]_{h}\right|^{2} d \alpha d h \mid \\
& +\left.\bar{c}_{3} r^{1 / 2}\left|\int_{0}^{l} \int\right||D|^{1 / 2}\left[\theta \ln z_{\alpha}\right]_{h} * \phi^{8 r h}\right|^{2} d \alpha d h\left|+K_{9}\right| l \mid
\end{aligned}
$$

where $\bar{c}_{1}, \bar{c}_{2}$, and $\bar{c}_{3}$ are constants depending on $m$ and $M$, and $K_{9}$ is independent of $l$. 
We now consider the term $r^{-1 / 2}\left|\int_{0}^{l}\right| \mathrm{II}_{1}|d h|$. We know

$$
\begin{aligned}
& \mathrm{II}_{1}=2 \iint \frac{\theta(\alpha) \theta(\beta)}{(\alpha-\beta)^{2}} \overline{\left(\frac{z_{h} * \phi^{r h}(\alpha)}{e^{\left[\ln z_{\alpha} * \phi^{r h}\right]_{h}(\alpha)}}-1\right)} \\
& \cdot\left(\frac{z_{h} * \phi^{r h}(\alpha)}{e^{\left[\ln z_{\alpha} * \phi^{r h}\right]_{h}(\alpha)}}-\frac{z_{h} * \phi^{r h}(\beta)}{e^{\left[\ln z_{\beta} * \phi^{r h}\right]_{h}(\beta)}}\right) d \alpha d \beta \\
& =-2 \iint \frac{\theta(\alpha) \theta^{\prime}(\beta)}{\alpha-\beta} \overline{\left(\frac{z_{h} * \phi^{r h}(\alpha)}{e^{\left[\ln z_{\alpha} * \phi^{r h}\right] h}(\alpha)}-1\right)} \\
& \cdot\left(\frac{z_{h} * \phi^{r h}(\alpha)}{e^{\left[\ln z_{\alpha} * \phi^{r h}\right]_{h}(\alpha)}}-\frac{z_{h} * \phi^{r h}(\beta)}{e^{\left[\ln z_{\beta} * \phi^{r h}\right] h}(\beta)}\right) d \alpha d \beta \\
& +2 \iint \frac{\theta(\alpha) \theta(\beta)}{\alpha-\beta} \overline{\left(\frac{z_{h} * \phi^{r h}(\alpha)}{e^{\left[\ln z_{\alpha} * \phi^{r h}\right]_{h}(\alpha)}}-1\right)} \partial_{\beta}\left(\frac{z_{h} * \phi^{r h}(\beta)}{e^{\left[\ln z_{\beta} * \phi^{r h}\right]_{h}(\beta)}}\right) d \alpha d \beta \\
& \leq K_{10}+2 \iint \frac{\theta(\alpha) \theta(\beta)}{\alpha-\beta} \overline{\left(\frac{z_{h} * \phi^{r h}(\alpha)}{e^{\left[\ln z_{\alpha} * \phi^{r h}\right] h}(\alpha)}-1\right)} \\
& \cdot \partial_{\beta}\left(\frac{z_{h} * \phi^{r h}(\beta)}{e^{\left[\ln z_{\beta} * \phi^{r h}\right]_{h}(\beta)}}\right) d \alpha d \beta,
\end{aligned}
$$

where $K_{10}$ depends on $m, M,|I|,\|\theta\|_{L^{\infty}}$, and $\left\|\theta^{\prime}\right\|_{L^{\infty}}$.

Now

$$
\begin{aligned}
& \partial_{\beta}\left(\frac{z_{h} * \phi^{r h}(\beta)}{e^{\left[\ln z_{\beta} * \phi^{r h}\right] h(\beta)}}\right) \\
& =\frac{1}{r h} \frac{z_{h} * \phi^{r h}(\beta)}{e^{\left[\ln z_{\beta} * \phi^{r h}\right] h(\beta)}}\left(\frac{z_{h} * \psi_{2}^{r h}(\beta)}{z_{h} * \phi^{r h}(\beta)}-\left[\frac{z_{\beta} * \psi_{2}^{r h}(\beta)}{z_{\beta} * \phi^{r h}(\beta)}\right]_{h}\right) \\
& =\frac{1}{r h^{2}} \int_{0}^{h} \frac{z_{h} * \phi^{r h}(\beta)}{e^{\left[\ln z_{\beta} * \phi^{r h}\right] h}(\beta)} z_{\beta} * \psi_{2}^{r h}(\beta+s) \\
& \cdot\left(\frac{1}{z_{h} * \phi^{r h}(\beta)}-\frac{1}{z_{\beta} * \phi^{r h}(\beta+s)}\right) d s,
\end{aligned}
$$

where $\psi_{2}$ is as in (3.63). Therefore,

$$
\mathrm{II}_{1} \leq K_{10}+\frac{2}{r h^{2}} \int_{0}^{h} \iint \frac{\theta(\alpha) \theta(\beta)}{\alpha-\beta} f(\alpha) g(\beta, s) h(\beta, s) d \alpha d \beta d s,
$$

where

$$
\begin{aligned}
f(\alpha) & =\overline{\left(\frac{z_{h} * \phi^{r h}(\alpha)}{e^{\left[\ln z_{\alpha} * \phi^{r h}\right]_{h}(\alpha)}}-1\right)}, \\
g(\beta, s) & =\frac{z_{h} * \phi^{r h}(\beta)}{e^{\left[\ln z_{\beta} * \phi^{r h}\right] h}(\beta)} z_{\beta} * \psi_{2}^{r h}(\beta+s), \\
h(\beta, s) & =\frac{1}{z_{h} * \phi^{r h}(\beta)}-\frac{1}{z_{\beta} * \phi^{r h}(\beta+s)} .
\end{aligned}
$$


From the antisymmetry of $1 /(\alpha-\beta)$ and by an interchange of the variables $\alpha$ and $\beta$, we have

$$
\begin{aligned}
\iint & \frac{\theta(\alpha) \theta(\beta)}{\alpha-\beta} f(\alpha) g(\beta, s) h(\beta, s) d \alpha d \beta \\
= & \frac{1}{2} \iint \frac{\theta(\alpha) \theta(\beta)}{\alpha-\beta} f(\alpha)(g(\beta, s)-g(\alpha, s)) h(\beta, s) d \alpha d \beta \\
& +\frac{1}{2} \iint \frac{\theta(\alpha) \theta(\beta)}{\alpha-\beta}(f(\alpha)-f(\beta)) g(\alpha, s) h(\beta, s) d \alpha d \beta \\
& +\frac{1}{2} \iint \frac{\theta(\alpha) \theta(\beta)}{\alpha-\beta} f(\beta) g(\alpha, s)(h(\beta, s)-h(\alpha, s)) d \alpha d \beta .
\end{aligned}
$$

Applying Theorem 2.6 and Proposition 2.7 to (3.69)-(3.70), we get that for $c_{0}$ sufficiently small and $\left\|\ln z_{\alpha}\right\|_{\mathrm{BMO}(4 I)} \leq c_{0}$,

$$
\begin{aligned}
\mathrm{II}_{1} \leq K_{10}+ & C \frac{\left\|\ln z_{\alpha}\right\|_{\mathrm{BMO}(4 I)}}{r h^{2}} \\
& \cdot \int_{0}^{h}\left(\|\theta f\|_{L^{2}(\mathbb{R})}\|\theta h\|_{L^{2}(\mathbb{R})}\right. \\
& \left.+\|\theta g\|_{L^{2}(\mathbb{R})}\|\theta h\|_{L^{2}(\mathbb{R})}+\|\theta f\|_{L^{2}(\mathbb{R})}\|\theta g\|_{L^{2}(\mathbb{R})}\right) d s \\
\leq K_{10}+ & C \frac{\left\|\ln z_{\alpha}\right\|_{\mathrm{BMO}(4 I)}}{r h^{2}} \\
& \cdot \int_{0}^{h}\left(\|\theta f\|_{L^{2}(\mathbb{R})}^{2}+\|\theta g(\cdot, s)\|_{L^{2}(\mathbb{R})}^{2}+\|\theta h(\cdot, s)\|_{L^{2}(\mathbb{R})}^{2}\right) d s
\end{aligned}
$$

where $C$ is a universal constant. Notice that

$$
|f(\alpha)| \leq c_{1} \frac{1}{h} \int_{0}^{h}\left|z_{\alpha} * \phi^{r h}(\alpha+s)-z_{h} * \phi^{r h}(\alpha)\right| d s,
$$

where $c_{1}$ is a constant that depends on $m$ and $M$. Using a result of the type of Lemma 3.8, we get

$$
\begin{aligned}
& \frac{1}{r h^{2}} \int_{0}^{h}\|\theta f\|_{L^{2}(\mathbb{R})}^{2} d s \\
& \leq c_{1} \frac{1}{r h^{2}} \int_{0}^{h} \int\left|\theta(\alpha)\left(z_{\alpha} * \phi^{r h}(\alpha+s)-z_{h} * \phi^{r h}(\alpha)\right)\right|^{2} d \alpha d s \\
& =c_{1} \frac{1}{r h^{2}} \int_{0}^{h} \int\left|\left(\left(\theta z_{\alpha}\right) * \phi^{r h}(\alpha+s)-\left[\theta z_{\alpha}\right]_{h} * \phi^{r h}(\alpha)\right)\right|^{2} d \alpha d s \\
& \quad+K_{11} .
\end{aligned}
$$


Furthermore, we have

$$
\begin{aligned}
& \frac{1}{r h^{2}} \int_{0}^{h}\|\theta g(\cdot, s)\|_{L^{2}(\mathbb{R})}^{2} d s \\
& \quad \leq \frac{c_{2}}{r h^{2}} \int_{0}^{h} \int\left|\theta(\alpha) z_{\alpha} * \psi_{2}^{r h}(\alpha+s)\right|^{2} d \alpha d s \\
& \quad=\frac{c_{2}}{r h^{2}} \int_{0}^{h} \int\left|\left(\theta z_{\alpha}\right) * \psi_{2}^{r h}(\alpha+s)\right|^{2} d \alpha d s+K_{12},
\end{aligned}
$$

and

$$
\begin{aligned}
& \frac{1}{r h^{2}} \int_{0}^{h}\|\theta h(\cdot, s)\|_{L^{2}(\mathbb{R})}^{2} d s \\
& \leq c_{3} \frac{1}{r h^{2}} \int_{0}^{h} \int\left|\theta(\alpha)\left(z_{\alpha} * \phi^{r h}(\alpha+s)-z_{h} * \phi^{r h}(\alpha)\right)\right|^{2} d \alpha d s \\
& =c_{3} \frac{1}{r h^{2}} \int_{0}^{h}\left|\left(\left(\theta z_{\alpha}\right) * \phi^{r h}(\alpha+s)-\left[\theta z_{\alpha}\right]_{h} * \phi^{r h}(\alpha)\right)\right|^{2} d \alpha d s \\
& \quad+K_{13},
\end{aligned}
$$

where $c_{2}$ and $c_{3}$ are constants that depend on $m$ and $M$, and $K_{11}, K_{12}$, and $K_{13}$ depend on $m, M,|I|,\|\theta\|_{L^{\infty}}$, and $\left\|\theta^{\prime}\right\|_{L^{\infty}}$. Summing up (3.71)-(3.74) and applying Plancherel's theorem, we obtain

$$
\begin{aligned}
r^{-1 / 2}\left|\int_{0}^{l} \mathrm{II}_{1} d h\right| \leq & c_{4} \frac{\left\|\ln z_{\alpha}\right\|_{\mathrm{BMO}(4 I)}}{r^{3 / 2}}\left(1+\ln r^{-1}\right) \\
& \left.\cdot\left|\int_{0}^{l} \int\right||D|^{1 / 2}\left[\theta z_{\alpha}\right]_{h}\right|^{2} d \alpha d h\left|+K_{14}\right| l \mid,
\end{aligned}
$$

where $c_{4}$ depends on $m$ and $M$, and $K_{14}$ is independent of $l$. ( $K_{14}$ depends on $m$, $M,|I|, r^{-1 / 2},\left\|\ln z_{\alpha}\right\|_{\mathrm{BMO}(4 I)},\|\theta\|_{L^{\infty}}$, and $\left\|\theta^{\prime}\right\|_{L^{\infty}}$.)

We need the following lemma before we proceed to estimate $r^{-1 / 2}\left|\int_{0}^{l} \mathrm{II}_{2} d h\right|$. Let $\phi$ and $z$ be as in Proposition 3.6, with $\left\|\ln z_{\alpha}\right\|_{\mathrm{BMO}(4 I)}$ satisfying (3.53).

LEMMA 3.13 There exists a constant $C$, independent of $h \neq 0$, such that

$$
\left\|\phi^{4 h} *\left(\ln z_{\alpha} * \phi^{h}-\ln z_{\alpha}\right)\right\|_{L^{\infty}(\mathbb{R})} \leq C\left\|\ln z_{\alpha}\right\|_{\mathrm{BMO}(4 I)} .
$$

$$
\left\|\ln z_{\alpha} * \phi^{h}\right\|_{L^{2}(4 I)} \leq C .
$$

PROOF:

(i) Without loss of generality, we assume that $h>0$. We know

$$
\ln z_{\alpha} * \phi^{h}-\ln z_{\alpha}=\int_{0}^{h} \frac{1}{t} \frac{z_{\alpha} * \psi^{t}}{z_{\alpha} * \phi^{t}} d t
$$


where

$$
\hat{\psi}(\xi)=\xi \hat{\phi}^{\prime}(\xi)
$$

Therefore

$$
\operatorname{supp} \hat{\psi}(t \xi) \subset\{1 \leq|t \xi| \leq 2\} .
$$

Let $\psi_{3} \in \mathfrak{S}(\mathbb{R})$ be such that $\hat{\psi}_{3} \in C_{0}^{\infty}(\mathbb{R})$ and

$$
\hat{\psi}_{3}(\xi)= \begin{cases}1 & \text { for } \frac{1}{2} \leq|\xi| \leq \frac{5}{2} \\ 0 & \text { for }|\xi| \leq \frac{1}{4} \text { or }|\xi| \geq 3\end{cases}
$$

Since for $|t| \leq|h|, \operatorname{supp} \hat{\phi}(4 h \xi) \subset\{4|h \xi| \leq 2\} \subset\left\{|t \xi| \leq \frac{1}{2}\right\}$, we have

$$
\begin{aligned}
\phi^{4 h} *\left(\ln z_{\alpha} * \phi^{h}-\ln z_{\alpha}\right) & =\int_{0}^{h} \frac{1}{t} \phi^{4 h} * \frac{z_{\alpha} * \psi^{t}}{z_{\alpha} * \phi^{t}} d t \\
& =\int_{0}^{h} \frac{1}{t} \phi^{4 h} *\left(z_{\alpha} * \psi^{t}\left(\frac{1}{z_{\alpha} * \phi^{t}}\right) * \psi_{3}^{t}\right) d t \\
& =\int_{0}^{h} \frac{1}{t} \phi^{4 h} *\left(z_{\alpha} * \psi^{t}\left(\frac{z_{\alpha} * \psi_{2}^{t}}{\left(z_{\alpha} * \phi^{t}\right)^{2}}\right) * \psi_{4}^{t}\right) d t,
\end{aligned}
$$

where $\psi_{2}$ is as in (3.63), and

$$
\hat{\psi}_{4}(\xi)=-\frac{\hat{\psi}_{3}(\xi)}{\xi}
$$

With an application of the Schwarz inequality and the Minkowski inequality, and the fact that for any functions $f$ and $g$,

$$
\begin{aligned}
|f * g(x)|^{2}=\left|\int f(x-y) g(y) d y\right|^{2} & \leq \int|g(y)| d y \int|f(x-y)|^{2}|g(y)| d y \\
& =\left(\int|g(y)| d y\right)|g| *|f|^{2},
\end{aligned}
$$

we get

$$
\begin{array}{rl}
\mid \phi^{4 h} & *\left(\ln z_{\alpha} * \phi^{h}-\ln z_{\alpha}\right) \mid \\
\leq & \left(\int_{0}^{h} \frac{1}{t}|\phi|^{4 h} *\left|z_{\alpha} * \psi^{t}\right|^{2} d t\right)^{1 / 2}\left(\int_{0}^{h} \frac{1}{t}|\phi|^{4 h} *\left|\left(\frac{z_{\alpha} * \psi_{2}^{t}}{\left(z_{\alpha} * \phi^{t}\right)^{2}}\right) * \psi_{4}^{t}\right|^{2} d t\right)^{1 / 2} \\
\leq & \left(\int_{0}^{h} \frac{1}{t}|\phi|^{4 h} *\left|z_{\alpha} * \psi^{t}\right|^{2} d t\right)^{1 / 2} \\
& \cdot\left(\int_{0}^{h} \frac{1}{t}\left(\int\left|\psi_{4}\right|^{t}(\alpha) d \alpha\right)|\phi|^{4 h} *\left|\psi_{4}\right|^{t} *\left|\left(\frac{z_{\alpha} * \psi_{2}^{t}}{\left(z_{\alpha} * \phi^{t}\right)^{2}}\right)\right|^{2} d t\right)^{1 / 2}
\end{array}
$$




$$
\begin{aligned}
\leq & \frac{4}{m^{2}} \int\left|\psi_{4}(\alpha)\right| d \alpha\left(\int_{0}^{h} \frac{1}{t}|\phi|^{4 h} *\left|z_{\alpha} * \psi^{t}\right|^{2} d t\right)^{1 / 2} \\
& \cdot\left(\int_{0}^{h} \frac{1}{t}|\phi|^{4 h} *\left|\psi_{4}\right|^{t} *\left|z_{\alpha} * \psi_{2}^{t}\right|^{2} d t\right)^{1 / 2},
\end{aligned}
$$

where in the last inequality, we used (3.52). Now (3.76) is just a direct consequence of Propositions 2.7 and 2.8, and the fact that for any $\psi \in \mathfrak{S}(\mathbb{R})$, with $\int \psi=0$ and $b \in \mathrm{BMO}(\mathbb{R})$,

$$
\left|b * \psi^{t}(x)\right|^{2} \frac{d x d t}{t}
$$

is a Carleson measure (see [25, p. 85]).

(ii) We know

$$
\ln z_{\alpha} * \phi^{h}-\phi^{4 h} * \ln z_{\alpha} * \phi^{h}=4 \psi_{1}^{4 h} *\left(\frac{z_{\alpha} * \psi_{2}^{h}}{z_{\alpha} * \phi^{h}}\right),
$$

where $\psi_{1}$ and $\psi_{2}$ are as in (3.62) and (3.63). Because $\psi_{i} \in L^{1}, i=1,2$, and we have (3.52), $\ln z_{\alpha} * \phi^{h}-\phi^{4 h} * \ln z_{\alpha} * \phi^{h} \in L^{\infty}(\mathbb{R})$, with $\| \ln z_{\alpha} * \phi^{h}-\phi^{4 h} * \ln z_{\alpha} *$ $\phi^{h} \|_{L^{\infty}} \leq c(m, M)$ for some constant $c(m, M)$ depending on $m$ and $M$. Assertion (ii) follows from (3.76) and the fact that $\ln z_{\alpha} \in L^{2}(4 I)$.

We are now ready to consider $r^{-1 / 2}\left|\int_{0}^{l} \mathrm{II}_{2} d h\right|$. From Lemma 3.8 and Lemma 3.13(ii), we know there is a constant $K_{15}$ such that

$$
\begin{aligned}
\mathrm{II}_{2} \leq \iint \frac{1}{(\alpha-\beta)^{2}} \mid \phi^{8 r h} *\left[\theta\left(\ln z_{\alpha} * \phi^{r h}-\ln z_{\alpha}\right)\right]_{h}(\alpha) \\
-\left.\phi^{8 r h} *\left[\theta\left(\ln z_{\beta} * \phi^{r h}-\ln z_{\beta}\right)\right]_{h}(\beta)\right|^{2} d \alpha d \beta+K_{15} .
\end{aligned}
$$

Using Fourier analysis and Plancherel's theorem, we obtain

$$
\begin{aligned}
\left|\int_{0}^{l} \mathrm{II}_{2} d h\right| \leq\left. c_{1}\left|\int_{0}^{l} \int\right| \xi|| \hat{\phi}(8 r h \xi)\right|^{2}\left|\frac{e^{i h \xi}-1}{h \xi}\right|^{2} \\
\cdot\left|\theta\left(\ln z_{\alpha} * \phi^{r h}-\ln z_{\alpha}\right)(\xi)\right|^{2} d \xi d h\left|+K_{15}\right| l \mid,
\end{aligned}
$$

where $c_{1}$ is a universal constant. Let

$$
\hat{F}(h \xi)=\int_{|h|}^{\infty}|\xi||\hat{\phi}(8 r t \xi)|^{2}\left|\frac{e^{i t \xi}-1}{t \xi}\right|^{2} d t=\int_{|h \xi|}^{\infty}|\hat{\phi}(8 r t)|^{2}\left|\frac{e^{i t}-1}{t}\right|^{2} d t
$$

(here we used the spherical symmetry of $\hat{\phi}$ ).

We have

$$
\operatorname{supp} \hat{F}(h \xi) \subset\{8 r|h \xi| \leq 2\}
$$


Therefore

$$
\begin{aligned}
& \left|\int_{0}^{l} \mathrm{II}_{2} d h\right| \\
& \leq\left. c_{1}\left|\iint_{0}^{l}\right| \theta\left(\ln z_{\alpha} \widehat{* \phi^{r h}}-\ln z_{\alpha}\right)(\xi)\right|^{2} d \hat{F}(h \xi) d \xi\left|+K_{15}\right| l \mid \\
& \leq 2 c_{1}\left|\iint_{0}^{l} \hat{F}(h \xi) \overline{\theta\left(\ln z_{\alpha} \widehat{* \phi^{r h}}-\ln z_{\alpha}\right)}(\xi) \theta \frac{z_{\alpha} * \psi^{r h}}{h z_{\alpha} * \phi^{r h}}(\xi) d h d \xi\right| \\
& +\left.c_{1}\left|\int \hat{F}(l \xi)\right| \theta\left(\ln z_{\alpha} \widehat{* \phi^{r l}}-\ln z_{\alpha}\right)(\xi)\right|^{2} d \xi\left|+K_{15}\right| l \mid,
\end{aligned}
$$

where $\psi$ is as in (3.79). Using the same calculation as that from (3.78) through (3.82), applying Lemma 3.12, and several times results in the same spirit as Lemma 3.8 , we get

$$
\begin{aligned}
& c_{1} \int \hat{F}(l \xi)\left|\theta\left(\ln z_{\alpha} * \widehat{\phi^{r l}}-\ln z_{\alpha}\right)(\xi)\right|^{2} d \xi \\
& =\left|\int F^{l} *\left(\theta\left(\ln z_{\alpha} * \phi^{r l}-\ln z_{\alpha}\right)\right)(\alpha) \overline{\theta\left(\ln z_{\alpha} * \phi^{r l}-\ln z_{\alpha}\right)}(\alpha) d \alpha\right| \\
& \leq\left|\int \theta(\alpha) F^{l} *\left(\ln z_{\alpha} * \phi^{r l}-\ln z_{\alpha}\right)(\alpha) \overline{\theta\left(\ln z_{\alpha} * \phi^{r l}-\ln z_{\alpha}\right)}(\alpha) d \alpha\right|+K_{16}|l| \\
& =\left|\int F^{l} *\left(\ln z_{\alpha} * \phi^{r l}-\ln z_{\alpha}\right)(\alpha) \theta^{2}(\alpha) \int_{0}^{l} \frac{1}{h} \overline{z_{\alpha} * \psi^{r h}} d h d \alpha\right|+K_{16}|l| \\
& \leq\left|\int_{0}^{l} \int F^{l} *\left(\ln z_{\alpha} * \phi^{r l}-\ln z_{\alpha}\right)(\alpha) \frac{1}{h} \overline{\left(\theta z_{\alpha}\right) * \psi^{r h}\left(\frac{\theta(\alpha)}{z_{\alpha} * \phi^{r h}}\right) * \psi_{3}^{r h}} d h d \alpha\right| \\
& +\bar{K}_{16}|l| \\
& \leq\left|\int_{0}^{l} \int F^{l} *\left(\ln z_{\alpha} * \phi^{r l}-\ln z_{\alpha}\right)(\alpha) \overline{\frac{1}{h}\left(\theta z_{\alpha}\right) * \psi^{r h}\left(\frac{\left(\theta z_{\alpha}\right) * \psi_{2}^{r h}}{\left(z_{\alpha} * \phi^{r h}\right)^{2}}\right) * \psi_{4}^{r h}} d h d \alpha\right| \\
& +\overline{\bar{K}}_{16}|l| \\
& \leq\left.\left. c_{2}\left\|\ln z_{\alpha}\right\|_{\mathrm{BMO}(4 I)}\left|\int_{0}^{l} \int \frac{1}{h}\right|\left(\theta z_{\alpha}\right) * \psi^{r h}\right|^{2} d \alpha d h\right|^{1 / 2} \\
& \left.\left.\cdot\left|\int_{0}^{l} \int \frac{1}{h}\right|\left(\theta z_{\alpha}\right) * \psi_{2}^{r h}\right|^{2} d \alpha d h\right|^{1 / 2}+\overline{\bar{K}}_{16}|l| \\
& \leq\left. c_{3}\left\|\ln z_{\alpha}\right\|_{\mathrm{BMO}(4 I)}\left|\int_{0}^{l} \int\right||D|^{1 / 2}\left[\theta z_{\alpha}\right]_{h}(\alpha)\right|^{2} d \alpha d h\left|+\overline{\bar{K}}_{16}\right| l \mid
\end{aligned}
$$

where $c_{2}$ and $c_{3}$ depend on $m, M$, and $\|F\|_{L^{1}(\mathbb{R})}$; consequently, $c_{2}$ and $c_{3}$ depend on $m, M$, and $r$; and $K_{16}, \bar{K}_{16}$, and $\overline{\bar{K}}_{16}$ are independent of $l$. In (3.86) we used the fact that $\hat{F}(l \xi)=\hat{F}(l \xi) \hat{\phi}(4 r l \xi)$ and therefore

$$
F^{l} *\left(\ln z_{\alpha} * \phi^{r l}-\ln z_{\alpha}\right)=F^{l} * \phi^{4 r l} *\left(\ln z_{\alpha} * \phi^{r l}-\ln z_{\alpha}\right) .
$$

The last step is a consequence of Plancherel's theorem. 
The calculation of

$$
c_{1}\left|\iint_{0}^{l} \hat{F}(h \xi) \overline{\theta\left(\ln z_{\alpha} \widehat{* \phi^{r h}}-\ln z_{\alpha}\right)}(\xi) \theta \frac{z_{\alpha} \widehat{* \psi^{r h}}}{h z_{\alpha} * \phi^{r h}}(\xi) d h d \xi\right|
$$

is similar. We have

$$
\begin{aligned}
& \left.c_{1} \mid \iint_{0}^{l} \hat{F}(h \xi) \overline{\theta\left(\ln z_{\alpha} * \phi^{r h}\right.}-\ln z_{\alpha}\right)(\xi) \theta \frac{z_{\alpha} * \psi^{r h}}{h z_{\alpha} * \phi^{r h}}(\xi) d h d \xi \mid \\
& =\left|\iint_{0}^{l} F^{h} *\left(\theta\left(\ln z_{\alpha} * \phi^{r h}-\ln z_{\alpha}\right)\right) \overline{\theta \frac{z_{\alpha} * \psi^{r h}}{h z_{\alpha} * \phi^{r h}}} d h d \alpha\right| \\
& \leq\left|\int_{0}^{l} \int F^{h} *\left(\ln z_{\alpha} * \phi^{r h}-\ln z_{\alpha}\right)(\alpha) \frac{1}{h}\left(\theta z_{\alpha}\right) * \psi^{r h}\left(\frac{\left(\theta z_{\alpha}\right) * \psi_{2}^{r h}}{\left(z_{\alpha} * \phi^{r h}\right)^{2}}\right) * \psi_{4}^{r h} d h d \alpha\right| \\
& +K_{17}|l| \\
& \leq\left. c_{3}\left\|\ln z_{\alpha}\right\|_{\mathrm{BMO}(4 I)}\left|\int_{0}^{l} \int\right||D|^{1 / 2}\left[\theta z_{\alpha}\right]_{h}(\alpha)\right|^{2} d \alpha d h\left|+K_{17}\right| l \mid
\end{aligned}
$$

where $c_{3}$ is the same as in (3.86), and $K_{17}$ is independent of $l$. Summing up (3.85) through (3.88), we obtain

$$
\begin{aligned}
r^{-1 / 2}\left|\int_{0}^{l} \mathrm{II}_{2} d h\right| \leq & 3 c_{3} r^{-1 / 2}\left\|\ln z_{\alpha}\right\|_{\mathrm{BMO}(4 I)} \\
& \left.\cdot\left|\int_{0}^{l} \int\right||D|^{1 / 2}\left[\theta z_{\alpha}\right]_{h}(\alpha)\right|^{2} d \alpha d h\left|+K_{18}\right| l \mid
\end{aligned}
$$

with $c_{3}$ is as in (3.86), and $K_{18}$ is independent of $l$.

Finally, we consider $\left|\int_{0}^{l}\right| I_{3}|d h|$ for $0<|l| \leq 1$. We let $\varphi^{h}=\left[\phi^{8 r h}\right]_{h}$ and $f=\ln z_{\alpha}$ just for convenience. We have for $0<|h| \leq 1$,

$$
\begin{aligned}
\left|I_{3}\right|=\mid \iint \frac{\theta(\alpha) \theta(\beta)}{(\alpha-\beta)^{2}}\left(e^{f * \varphi^{h}(\alpha)-f * \varphi^{h}(\beta)}+e^{f * \varphi^{h}(\beta)-f * \varphi^{h}(\alpha)}\right. \\
\left.-2-\left(f * \varphi^{h}(\alpha)-f * \varphi^{h}(\beta)\right)^{2}\right) d \alpha d \beta \mid \\
\leq 2 \mid \int_{h}^{1} \iint \frac{\theta(\alpha) \theta(\beta)}{(\alpha-\beta)^{2}}\left(\frac{e^{f * \varphi^{t}(\alpha)}}{e^{f * \varphi^{t}(\beta)}}-\frac{e^{f * \varphi^{t}(\beta)}}{e^{f * \varphi^{t}(\alpha)}}-2\left(f * \varphi^{t}(\alpha)-f * \varphi^{t}(\beta)\right)\right) \\
+\frac{d}{d t} \varphi^{t} * f(\alpha) d \alpha d \beta d t \mid \\
+\mid \iint \frac{\theta(\alpha) \theta(\beta)}{(\alpha-\beta)^{2}}\left(e^{f * \varphi^{1}(\alpha)-f * \varphi^{1}(\beta)}+e^{f * \varphi^{1}(\beta)-f * \varphi^{1}(\alpha)}\right. \\
\left.-2-\left(f * \varphi^{1}(\alpha)-f * \varphi^{1}(\beta)\right)^{2}\right) d \alpha d \beta \mid
\end{aligned}
$$




$$
\begin{gathered}
\leq 2 \mid \int_{h}^{1} \iint_{\frac{\theta(\alpha) \theta^{\prime}(\beta)}{\alpha-\beta}}\left(\frac{e^{f * \varphi^{t}(\alpha)}}{e^{f * \varphi^{t}(\beta)}}-\frac{e^{f * \varphi^{t}(\beta)}}{e^{f * \varphi^{t}(\alpha)}}-2\left(f * \varphi^{t}(\alpha)-f * \varphi^{t}(\beta)\right)\right) \\
\cdot \frac{d}{d t} \varphi^{t} * f(\alpha) d \alpha d \beta d t \mid \\
+2 \mid \int_{h}^{1} \iint \frac{\theta(\alpha) \theta(\beta)}{\alpha-\beta}\left(\frac{e^{f * \varphi^{t}(\alpha)}}{e^{f * \varphi^{t}(\beta)}}+\frac{e^{f * \varphi^{t}(\beta)}}{e^{f * \varphi^{t}(\alpha)}}-2\right) \\
\cdot \partial_{\beta} \varphi^{t} * f(\beta) \frac{d}{d t} \varphi^{t} * f(\alpha) d \alpha d \beta d t \mid+K_{19}
\end{gathered}
$$

Therefore

$$
\begin{aligned}
\left|I_{3}\right| \leq & c_{1}\|f\|_{\mathrm{BMO}(4 I)}\left\|\theta^{\prime}\right\|_{L^{2}}\left|\int_{h}^{1}\left\|\theta \frac{d}{d t} \varphi^{t} * f\right\|_{L^{2}(\mathbb{R})} d t\right| \\
& +c_{2}\|f\|_{\mathrm{BMO}(4 I)}\left|\int_{h}^{1}\left\|\theta \partial_{\beta} \varphi^{t} * f\right\|_{L^{2}(\mathbb{R})}\left\|\theta \frac{d}{d t} \varphi^{t} * f\right\|_{L^{2}(\mathbb{R})} d t\right|+K_{19} \\
\leq & \left.c_{3}\|f\|_{\mathrm{BMO}(4 I)}\left|\int_{h}^{1} \int\right| \theta(\alpha) \frac{d}{d t} \varphi^{t} * f(\alpha)\right|^{2} d \alpha d t \mid \\
& +\left.c_{4}\|f\|_{\mathrm{BMO}(4 I)}\left|\int_{h}^{1} \int\right| \theta(\alpha) \partial_{\alpha} \varphi^{t} * f(\alpha)\right|^{2} d \alpha d t \mid+\bar{K}_{19} \\
\leq & \left.c_{3}\|f\|_{\mathrm{BMO}(4 I)}\left|\int_{h}^{1} \int\right| \frac{d}{d t} \varphi^{t} *(\theta f)(\alpha)\right|^{2} d \alpha d t \mid \\
& +\left.c_{4}\|f\|_{\mathrm{BMO}(4 I)}\left|\int_{h}^{1} \int\right| \partial_{\alpha} \varphi^{t} *(\theta f)(\alpha)\right|^{2} d \alpha d t \mid+\overline{\bar{K}}_{19 .} .
\end{aligned}
$$

Here in (3.90)-(3.91) we used integration by parts, Theorem 2.6, and results in the same spirit as Lemma 3.8. Constants $c_{1}, c_{2}, c_{3}$, and $c_{4}$ depend on $m$ and $M$, and $K_{19}, \bar{K}_{19}$, and $\overline{\bar{K}}_{19}$ are independent of $h$. It follows from Plancherel's theorem that

$$
\begin{aligned}
& \left|\int_{0}^{l}\right| I_{3}|d h| \\
& \quad \leq\left. c_{5}\left\|\ln z_{\alpha}\right\|_{\text {ВмО }(4 I) \mid}\left|\int_{0}^{l} \int\right||D|^{1 / 2}\left[\theta \ln z_{\alpha}\right]_{h} * \phi^{8 r h}\right|^{2} d h\left|+K_{20}\right| l \mid
\end{aligned}
$$

where $c_{5}$ depends on $m, M$, and $r$, and $K_{20}$ is independent of $l$.

Summing up the calculation from (3.52) through (3.92), we obtain Proposition 3.6.

\section{$4 H^{2}$ Regularity of $z_{\alpha}$ and $H^{3 / 2}$ Compatibility of the Initial Data}

In this section, we prove the following result:

Proposition 4.1 Assume that $z \in H^{1}\left([0, T], L_{\mathrm{loc}}^{2}(\mathbb{R})\right) \cap L^{2}\left([0, T], H_{\mathrm{loc}}^{1}(\mathbb{R})\right)$ is a solution of the Birkhoff-Rott equation (1.6) for $0 \leq t \leq T$, satisfying assumption (i) of Theorem 1.2. Then there exists a constant $c_{1}(m, M)>0$ such that if on some 
fixed interval $(a, b)$ there exists a $\delta_{0}>0$ such that for some determination of the multivalued function $\ln z_{\alpha}$ we have

$$
\sup _{[0, T]}\left\|\ln z_{\alpha}(\cdot, t)\right\|_{\operatorname{BMO}(a, b), \delta_{0}} \leq c_{1}(m, M),
$$

then $z_{\alpha} \in H^{2}(K)$ for every compact rectangle $K$ of $(a, b) \times(0, T)$, and $z_{\alpha} \in$ $C((a, b) \times(0, T))$. If, in addition, $\operatorname{Im}\left((1+i) \ln z_{\alpha}(\cdot, 0)\right) \in H_{\mathrm{loc}}^{3 / 2}(a, b)$, then $\operatorname{Re}((1+$ $\left.i) \ln z_{\alpha}(\cdot, 0)\right) \in H_{\mathrm{loc}}^{3 / 2}(a, b)$, and $z_{\alpha}(\cdot, 0) \in H_{\mathrm{loc}}^{3 / 2}(a, b)$; moreover, $z_{\alpha} \in H^{2}(K)$ for every compact rectangle $K$ of $(a, b) \times[0, T)$, and $z_{\alpha} \in C((a, b) \times[0, T))$.

In order to prove Proposition 4.1, we need the following lemma:

Lemma 4.2 Assume that $\delta=\delta(t) \in C^{0,1}(\mathbb{R})$ and $\operatorname{supp} \delta \subset\left[T_{1}, T_{2}\right]$. Then for any $u=u(\alpha, t) \in C^{1}\left(\mathbb{R}^{2}\right)$ such that $u$ is compactly supported in $\alpha$ for each fixed $t$, we have

$$
\begin{aligned}
& \iint \delta^{2}(t) u^{4}(\alpha, t) d \alpha d t \\
& \leq 3\left(\iint u^{2}(\alpha, t) \chi_{\left[T_{1}, T_{2}\right]}(t) d \alpha d t\right)\left(\iint \delta^{2}(t)\left(u_{\alpha}^{2}+u_{t}^{2}\right) d \alpha d t\right) \\
& \quad+\left\|\delta^{\prime}\right\|_{L^{\infty}}^{2}\left(\iint u^{2}(\alpha, t) \chi_{\left[T_{1}, T_{2}\right]}(t) d \alpha d t\right)^{2}
\end{aligned}
$$

PROOF: The proof is a modification of that in Ladyzhenskaya [30]. Since $u$ is compactly supported in $\alpha$, we have that

$$
\max _{\alpha} u^{2}(\alpha, t) \leq 2 \int\left|u(\alpha, t) u_{\alpha}(\alpha, t)\right| d \alpha .
$$

Therefore

$$
\begin{aligned}
\iint & \delta^{2}(t) u^{4}(\alpha, t) d \alpha d t \\
\leq & \int \delta^{2}(t) \max _{\alpha} u^{2}(\alpha, t)\left(\int u^{2}(\alpha, t) d \alpha\right) d t \\
\leq & 2\left(\iint \delta(t)\left|u(\alpha, t) u_{\alpha}(\alpha, t)\right| d \alpha d t\right) \max _{t} \int \delta(t) u^{2}(\alpha, t) d \alpha \\
\leq & 2\left(\iint u^{2} \chi_{\left[T_{1}, T_{2}\right]}(t) d \alpha d t\right)^{1 / 2}\left(\iint \delta^{2}(t) u_{\alpha}^{2} d \alpha d t\right)^{1 / 2} \\
& \cdot \iint\left(\left|\delta^{\prime}(t)\right| u^{2}+2\left|u u_{t} \delta(t)\right|\right) d \alpha d t \\
\leq & 2\left(\iint u^{2}(\alpha, t) \chi_{\left[T_{1}, T_{2}\right]}(t) d \alpha d t\right)\left(\iint \delta^{2}(t)\left(u_{\alpha}^{2}+u_{t}^{2}\right) d \alpha d t\right) \\
& +2\left\|\delta^{\prime}\right\|_{L^{\infty}}\left(\iint u^{2}(\alpha, t) \chi_{\left[T_{1}, T_{2}\right]} d \alpha d t\right)^{3 / 2}\left(\iint \delta^{2}(t) u_{\alpha}^{2} d \alpha d t\right)^{1 / 2} .
\end{aligned}
$$


In (4.2) we used Hölder's inequality and the geometric inequality. Inequality (4.1) directly follows from (4.2) by a further application of the geometric inequality.

We record here some identities that will be useful in the proof of Proposition 4.1. The proof is straightforward; we omit it.

LEMMA 4.3 For any function $f=f(\beta), f$ differentiable, we have

$$
\begin{aligned}
& f_{h}(\beta)-\left[f_{h}\right]_{h}(\beta)= \\
& \frac{1}{h^{2}} \int_{0}^{h} k^{2} f_{k k}(\beta+h) d k-\frac{1}{h^{2}} \int_{0}^{h} k^{2} f_{k k}(\beta) d k-\frac{1}{2} \int_{0}^{h} f_{h h}(\beta+k) d k, \\
& \quad \frac{1}{f_{\beta}(\beta)}-\frac{2}{f_{h}(\beta)}+\frac{1}{f_{2 h}(\beta)} \\
& \quad=\left(2 f_{h}(\beta)-f_{\beta}(\beta)-f_{2 h}(\beta)\right)\left(\frac{2}{f_{\beta}(\beta) f_{h}(\beta)}-\frac{1}{f_{\beta}(\beta) f_{2 h}(\beta)}\right) . \\
& \quad-\frac{1}{2} \frac{h^{2} f_{h h}(\beta)^{2}}{f_{\beta}(\beta) f_{h}(\beta) f_{2 h}(\beta)}
\end{aligned}
$$

We again use a difference quotient argument to prove Proposition 4.1. We begin with $c_{1}(m, M)=c(m, M)$, where $c(m, M)$ is as defined in (3.34). We may need to take $c_{1}(m, M)$ smaller than $c(m, M)$ in order to obtain the conclusions of Proposition 4.1 .

For any given closed interval $[c, d] \subset(a, b)$, let $\delta_{1}$ be as defined in Lemma 3.4 and $Q$ any interval satisfying $2 Q \subset[c, d],|2 Q| \leq \frac{1}{2} \delta_{1} \leq \frac{1}{4} \delta_{0}$. Take $\eta, \rho \in C_{0}^{\infty}(\mathbb{R})$ such that

$$
\begin{array}{ll}
\eta(\alpha)=1 \text { on } \frac{9}{8} Q, & \operatorname{supp} \eta \subset \frac{5}{4} Q, \\
\rho(\alpha)=1 \text { on } \frac{3}{2} Q, & \operatorname{supp} \rho \subset \frac{15}{8} Q,
\end{array}
$$

and $0 \leq \eta(\alpha), \rho(\alpha) \leq 1,\left|\eta^{\prime}(\alpha)\right|+\left|\rho^{\prime}(\alpha)\right| \leq C /|Q|,\left|\eta^{\prime \prime}(\alpha)\right|+\left|\rho^{\prime \prime}(\alpha)\right| \leq C /|Q|^{2}$ for some constant $C>0$. For $h \neq 0$, we want to estimate the $L_{\text {loc }}^{2}$-norm of

$$
z_{\alpha h h}=\frac{1}{h^{2}}\left(z_{\alpha}(\alpha+2 h, t)+z_{\alpha}(\alpha, t)-2 z_{\alpha}(\alpha+h, t)\right)
$$

and

$$
z_{t h h}=\frac{1}{h^{2}}\left(z_{t}(\alpha+2 h, t)+z_{t}(\alpha, t)-2 z_{t}(\alpha+h, t)\right)
$$


Let $0<|h| \leq \frac{1}{24}|Q|$. We have from (1.6) that

$$
\begin{aligned}
& 2 \pi i \bar{z}_{t h h}(\alpha, t) \\
& \begin{array}{l}
=\int \frac{z_{\beta}(\beta+h, t)}{z(\alpha+h, t)-z(\beta+h, t)}\left(\frac{1}{z_{\beta}}\right)_{h h}(\beta, t) \rho(\beta) d \beta \\
\quad+\frac{1}{h^{2}} \int\left(\frac{z_{\beta}(\beta, t)}{z(\alpha, t)-z(\beta, t)}-\frac{z_{\beta}(\beta+h, t)}{z(\alpha+h, t)-z(\beta+h, t)}\right) \frac{\rho(\beta)}{z_{\beta}(\beta, t)} d \beta \\
\quad+\frac{1}{h^{2}} \int\left(\frac{z_{\beta}(\beta+2 h, t)}{z(\alpha+2 h, t)-z(\beta+2 h, t)}-\frac{z_{\beta}(\beta+h, t)}{z(\alpha+h, t)-z(\beta+h, t)}\right) \\
\quad \cdot \frac{\rho(\beta)}{z_{\beta}(\beta+2 h, t)} d \beta \\
\quad+\frac{1}{h^{2}} \int\left(\frac{1}{z(\alpha+2 h, t)-z(\beta+2 h, t)}+\frac{1}{z(\alpha, t)-z(\beta, t)}-\frac{2}{z(\alpha+h, t)-z(\beta+h, t)}\right) \\
=\int \frac{1}{\alpha-\beta}\left(\frac{1}{z_{\beta}}\right)_{h h}(\beta, t) \rho(\beta) d \beta+\mathrm{I}+\mathrm{II}+\mathrm{III}+\mathrm{IV},
\end{array}
\end{aligned}
$$

where

$$
\begin{aligned}
& \mathrm{I}=\int\left(\frac{z_{\beta}(\beta+h, t)}{z(\alpha+h, t)-z(\beta+h, t)}-\frac{1}{\alpha-\beta}\right)\left(\frac{1}{z_{\beta}}\right)_{h h}(\beta, t) \rho(\beta) d \beta, \\
& \mathrm{II}=\frac{1}{h^{2}} \int\left(\frac{z_{\beta}(\beta, t)}{z(\alpha, t)-z(\beta, t)}-\frac{z_{\beta}(\beta+h, t)}{z(\alpha+h, t)-z(\beta+h, t)}\right) \frac{\rho(\beta)}{z_{\beta}(\beta, t)} d \beta, \\
& \mathrm{III}=\frac{1}{h^{2}} \int\left(\frac{z_{\beta}(\beta+2 h, t)}{z(\alpha+2 h, t)-z(\beta+2 h, t)}-\frac{z_{\beta}(\beta+h, t)}{z(\alpha+h, t)-z(\beta+h, t)}\right) \frac{\rho(\beta)}{z_{\beta}(\beta+2 h, t)} d \beta, \\
& \mathrm{IV}=\frac{1}{h^{2}} \int\left(\frac{1}{z(\alpha+2 h, t)-z(\beta+2 h, t)}+\frac{1}{z(\alpha, t)-z(\beta, t)}-\frac{2}{z(\alpha+h, t)-z(\beta+h, t)}\right) \\
& \cdot(1-\rho(\beta)) d \beta .
\end{aligned}
$$

Define

$$
G_{2}(\alpha, t)=\mathrm{I}+\mathrm{II}+\mathrm{III}+\mathrm{IV} .
$$

Therefore

$$
\begin{aligned}
& 2 \pi i \eta(\alpha) \bar{z}_{t h h}(\alpha, t) \\
& \quad=\int \frac{\eta(\beta)}{\alpha-\beta}\left(\frac{1}{z_{\beta}}\right)_{h h}(\beta, t) d \beta+[\eta, H]\left\{\rho\left(\frac{1}{z_{\alpha}}\right)_{h h}\right\}+\eta(\alpha) G_{2}(\alpha, t) .
\end{aligned}
$$

We first estimate $\int\left|\eta(\alpha) G_{2}(\alpha, t)\right|^{2} d \alpha$ for fixed $t \in[0, T]$. Notice that the term $\eta(\alpha) \mathrm{I}$ can be readily estimated using Corollary 2.4. We need to calculate further II, 
III, and IV. For II, we write

$$
\begin{aligned}
& \mathrm{II}= \frac{1}{h^{2}} \int\left(\frac{z_{\beta}(\beta, t)}{z(\alpha, t)-z(\beta, t)}-\frac{z_{\beta}(\beta+h, t)}{z(\alpha+h, t)-z(\beta+h, t)}\right) \rho(\beta)\left[\frac{1}{z_{h}}\right]_{h}(\beta, t) d \beta \\
&+ \frac{1}{h^{2}} \int\left(\frac{z_{\beta}(\beta, t)}{z(\alpha, t)-z(\beta, t)}-\frac{z_{\beta}(\beta+h, t)}{z(\alpha+h, t)-z(\beta+h, t)}\right) \rho(\beta) \\
& \cdot\left(\frac{1}{z_{\beta}}-\frac{2}{z_{h}}+\frac{1}{z_{2 h}}\right)(\beta, t) d \beta \\
&+\frac{1}{h^{2}} \int\left(\frac{z_{\beta}(\beta, t)}{z(\alpha, t)-z(\beta, t)}-\frac{z_{\beta}(\beta+h, t)}{z(\alpha+h, t)-z(\beta+h, t)}\right) \rho(\beta) \\
& \\
&+\left(\frac{1}{z_{h}(\beta, t)}-\left[\frac{1}{z_{h}}\right]_{h}(\beta, t)\right) d \beta \\
&+\frac{1}{h^{2}} \int\left(\frac{z_{\beta}(\beta, t)}{z(\alpha, t)-z(\beta, t)}-\frac{z_{\beta}(\beta+h, t)}{z(\alpha+h, t)-z(\beta+h, t)}\right) \rho(\beta) \\
& \quad \cdot\left(\frac{1}{z_{h}(\beta, t)}-\frac{1}{z_{2 h}(\beta, t)}\right) d \beta \\
&=\mathrm{II}_{1}+\mathrm{II}_{2}+\mathrm{I}_{3}+\mathrm{II}_{4} .
\end{aligned}
$$

Notice that $\eta(\alpha) \mathrm{II}_{2}$ can be estimated using identities (4.3) and (4.5), Lemma 4.2, and Corollary 2.4. We use integration by parts on $\mathrm{II}_{3}$ and $\mathrm{II}_{4}$ and the fact that $f(h)-f(0)=\int_{0}^{h} f^{\prime}(\gamma) d \gamma$ for an absolutely continuous function $f$. We get

$$
\begin{aligned}
& \mathrm{I}_{3}=\frac{1}{h^{2}} \int\left(\frac{z_{\beta}(\beta, t)}{z(\alpha, t)-z(\beta, t)}-\frac{z_{\beta}(\beta+h, t)}{z(\alpha+h, t)-z(\beta+h, t)}\right) \rho(\beta) \\
& \cdot\left(\frac{1}{z_{h}(\beta, t)}-\left[\frac{1}{z_{h}}\right]_{h}(\beta, t)\right) d \beta \\
&=-\frac{1}{h^{2}} \int \ln \frac{z(\alpha+h, t)-z(\beta+h, t)}{z(\alpha, t)-z(\beta, t)} \partial_{\beta}\left\{\rho(\beta)\left(\frac{1}{z_{h}(\beta, t)}-\left[\frac{1}{z_{h}}\right]_{h}(\beta, t)\right)\right\} d \beta \\
&=-\frac{1}{h^{2}} \int_{0}^{h} \int \frac{z_{\gamma}(\alpha+\gamma, t)-z_{\gamma}(\beta+\gamma, t)}{z(\alpha+\gamma, t)-z(\beta+\gamma, t)} \\
& \cdot \partial_{\beta}\left\{\rho(\beta)\left(\frac{1}{z_{h}(\beta, t)}-\left[\frac{1}{z_{h}}\right]_{h}(\beta, t)\right)\right\} d \beta d \gamma,
\end{aligned}
$$

and

$$
\begin{aligned}
& \mathrm{II}_{4}=\frac{1}{h^{2}} \int\left(\frac{z_{\beta}(\beta, t)}{z(\alpha, t)-z(\beta, t)}-\frac{z_{\beta}(\beta+h, t)}{z(\alpha+h, t)-z(\beta+h, t)}\right) \\
& \cdot \rho(\beta)\left(\frac{1}{z_{h}(\beta, t)}-\frac{1}{z_{2 h}(\beta, t)}\right) d \beta \\
&=-\frac{1}{h^{2}} \int_{0}^{h} \int \frac{z_{\gamma}(\alpha+\gamma, t)-z_{\gamma}(\beta+\gamma, t)}{z(\alpha+\gamma, t)-z(\beta+\gamma, t)} \\
& \cdot \partial_{\beta}\left\{\rho(\beta)\left(\frac{1}{z_{h}(\beta, t)}-\frac{1}{z_{2 h}(\beta, t)}\right)\right\} d \beta d \gamma,
\end{aligned}
$$


where

$$
\begin{aligned}
\partial_{\beta}\left\{\rho(\beta)\left(\frac{1}{z_{h}(\beta, t)}-\left[\frac{1}{z_{h}}\right]_{h}(\beta, t)\right)\right\} \\
=\rho^{\prime}(\beta)\left(\frac{1}{z_{h}(\beta, t)}-\left[\frac{1}{z_{h}}\right]_{h}(\beta, t)\right) \\
\quad+\rho(\beta)\left(-\frac{z_{\beta h}(\beta, t)}{z_{h}^{2}(\beta, t)}+\frac{z_{h h}(\beta, t)}{z_{h}(\beta, t) z_{h}(\beta+h, t)}\right) \\
=\rho^{\prime}(\beta)\left(\frac{1}{z_{h}(\beta, t)}-\left[\frac{1}{z_{h}}\right]_{h}(\beta, t)\right) \\
\quad+\rho(\beta)\left(\frac{-z_{\beta h}(\beta, t)+z_{h h}(\beta, t)}{z_{h}^{2}(\beta, t)}-\frac{h z_{h h}^{2}(\beta, t)}{z_{h}^{2}(\beta, t) z_{h}(\beta+h, t)}\right)
\end{aligned}
$$

and

$$
\begin{aligned}
\partial_{\beta}\{ & \left.\rho(\beta)\left(\frac{1}{z_{h}(\beta, t)}-\frac{1}{z_{2 h}(\beta, t)}\right)\right\} \\
= & \rho^{\prime}(\beta)\left(\frac{1}{z_{h}(\beta, t)}-\frac{1}{z_{2 h}(\beta, t)}\right)+\rho(\beta)\left(-\frac{z_{\beta h}(\beta, t)}{z_{h}^{2}(\beta, t)}+\frac{\partial_{\beta} z_{2 h}(\beta, t)}{z_{2 h}^{2}(\beta, t)}\right) \\
= & \rho^{\prime}(\beta)\left(\frac{1}{z_{h}(\beta, t)}-\frac{1}{z_{2 h}(\beta, t)}\right)+\frac{h}{2} \rho(\beta) \frac{z_{\beta h h}(\beta, t)}{z_{2 h}^{2}(\beta, t)} \\
& -\rho(\beta)\left(z_{\beta h}(\beta, t)-z_{h h}(\beta, t)\right) \frac{h z_{h h}(\beta, t)\left(z_{h}(\beta, t)+z_{2 h}(\beta, t)\right)}{2 z_{h}^{2}(\beta, t) z_{2 h}^{2}(\beta, t)} \\
& -\frac{h}{2} \rho(\beta) \frac{z_{h h}^{2}(\beta, t)\left(z_{h}(\beta, t)+z_{2 h}(\beta, t)\right)}{z_{h}^{2}(\beta, t) z_{2 h}^{2}(\beta, t)} .
\end{aligned}
$$

Therefore $\eta(\alpha) \mathrm{II}_{3}$ and $\eta(\alpha) \mathrm{II}_{4}$ can be estimated through identity (4.4), Lemma 4.2, and Theorem 2.6. We can handle III in a similar way by rewriting:

$$
\begin{aligned}
& \frac{\rho(\beta)}{z_{\beta}(\beta+2 h, t)} \\
& =\rho(\beta+h)\left[\frac{1}{z_{h}}\right]_{h}(\beta+h, t) \\
& \quad+\rho(\beta)\left(\frac{1}{z_{\beta}(\beta+2 h, t)}-\frac{2}{z_{h}(\beta+2 h, t)}+\frac{1}{z_{2 h}(\beta+2 h, t)}\right) \\
& \quad+\rho(\beta)\left(\frac{1}{z_{h}(\beta+2 h, t)}-\left[\frac{1}{z_{h}}\right]_{h}(\beta+2 h, t)\right) \\
& \quad+\rho(\beta)\left(\frac{1}{z_{h}(\beta+2 h, t)}-\frac{1}{z_{2 h}(\beta+2 h, t)}\right) \\
& \quad+\rho(\beta)\left[\frac{1}{z_{h}}\right]_{h}(\beta+2 h, t)-\rho(\beta+h)\left[\frac{1}{z_{h}}\right]_{h}(\beta+h, t) .
\end{aligned}
$$


Notice that the terms in III corresponding to the second, third, and fourth terms on the right-hand side of (4.13) can be estimated in the same way as $\mathrm{II}_{2}, \mathrm{II}_{3}$, and $\mathrm{II}_{4}$. For the last term

$$
\begin{aligned}
\mathrm{III}_{5}=\frac{1}{h^{2}} \int & \left(\frac{z_{\beta}(\beta+2 h, t)}{z(\alpha+2 h, t)-z(\beta+2 h, t)}-\frac{z_{\beta}(\beta+h, t)}{z(\alpha+h, t)-z(\beta+h, t)}\right) \\
& \cdot\left(\rho(\beta)\left[\frac{1}{z_{h}}\right]_{h}(\beta+2 h, t)-\rho(\beta+h)\left[\frac{1}{z_{h}}\right]_{h}(\beta+h, t)\right) d \beta,
\end{aligned}
$$

we use integration by parts. We have

$$
\begin{aligned}
\mathrm{III}_{5}=\frac{1}{h^{2}} \int_{h}^{2 h} \int \frac{z_{\gamma}(\alpha+\gamma, t)-z_{\gamma}(\beta+\gamma, t)}{z(\alpha+\gamma, t)-z(\beta+\gamma, t)} \\
\quad \cdot \partial_{\beta}\left\{\rho(\beta)\left[\frac{1}{z_{h}}\right]_{h}(\beta+2 h, t)-\rho(\beta+h)\left[\frac{1}{z_{h}}\right]_{h}(\beta+h, t)\right\} d \beta d \gamma,
\end{aligned}
$$

where

$$
\begin{aligned}
\partial_{\beta}\left\{\rho(\beta)\left[\frac{1}{z_{h}}\right]_{h}(\beta+2 h, t)-\rho(\beta+h)\left[\frac{1}{z_{h}}\right]_{h}(\beta+h, t)\right\} \\
=\rho^{\prime}(\beta)\left[\frac{1}{z_{h}}\right]_{h}(\beta+2 h, t)-\rho^{\prime}(\beta+h)\left[\frac{1}{z_{h}}\right]_{h}(\beta+h, t) \\
\quad+\rho(\beta)\left(\frac{1}{z_{h}}\right)_{h}(\beta+2 h, t)-\rho(\beta+h)\left(\frac{1}{z_{h}}\right)_{h}(\beta+h, t) \\
=\rho^{\prime}(\beta)\left(\left[\frac{1}{z_{h}}\right]_{h}(\beta+2 h, t)-\left[\frac{1}{z_{h}}\right]_{h}(\beta+h, t)\right) \\
\quad+\left(\rho^{\prime}(\beta)-\rho^{\prime}(\beta+h)\right)\left[\frac{1}{z_{h}}\right]_{h}(\beta+h, t)+h \rho(\beta)\left(\frac{1}{z_{h}}\right)_{h h}(\beta+h, t) \\
\quad+(\rho(\beta)-\rho(\beta+h))\left(\frac{1}{z_{h}}\right)_{h}(\beta+h, t) .
\end{aligned}
$$

Therefore $\eta(\alpha) \mathrm{III}_{5}$ can also be estimated through Lemma 4.2 and Theorem 2.6. The term $\eta(\alpha)$ IV is a "good term" and can be estimated through rewriting IV as

$$
\begin{aligned}
\mathrm{IV}= & \frac{1}{h^{2}} \int\left(\frac{1}{z(\alpha+2 h, t)-z(\beta+h, t)}-\frac{1}{z(\alpha+h, t)-z(\beta, t)}\right)(1-\rho(\beta-h)) d \beta \\
& +\frac{1}{h^{2}} \int\left(\frac{1}{z(\alpha, t)-z(\beta, t)}-\frac{1}{z(\alpha+h, t)-z(\beta+h, t)}\right)(1-\rho(\beta)) d \beta \\
= & \int \frac{-z_{h}(\alpha+h, t)+z_{h}(\beta, t)}{(z(\alpha+2 h, t)-z(\beta+h, t))(z(\alpha+h, t)-z(\beta, t))} \frac{\rho(\beta)-\rho(\beta-h)}{h} d \beta \\
& -\int \frac{z_{h h}(\alpha, t)}{(z(\alpha+2 h, t)-z(\beta+h, t))(z(\alpha+h, t)-z(\beta, t))}(1-\rho(\beta)) d \beta \\
& +\int \frac{\left(z_{h}(\alpha, t)-z_{h}(\beta, t)\right) z_{h}(\alpha+h, t)(1-\rho(\beta))}{(z(\alpha+h, t)-z(\beta, t))(z(\alpha+2 h, t)-z(\beta+h, t))(z(\alpha+h, t)-z(\beta+h, t))} d \beta \\
& +\int \frac{\left(z_{h}(\alpha, t)-z_{h}(\beta, t)\right) z_{h}(\alpha, t)(1-\rho(\beta))}{(z(\alpha+h, t)-z(\beta+h, t))(z(\alpha+h, t)-z(\beta, t))(z(\alpha, t)-z(\beta, t))} d \beta .
\end{aligned}
$$


What is left to be considered among the terms I $+\mathrm{II}+\mathrm{III}+\mathrm{IV}$ is $\mathrm{II}_{1}+\mathrm{III}_{1}$ :

$$
\begin{aligned}
& \mathrm{II}_{1}+ \mathrm{II}_{1} \\
&= \frac{1}{h^{2}} \int\left(\frac{z_{\beta}(\beta, t)}{z(\alpha, t)-z(\beta, t)}-\frac{z_{\beta}(\beta+h, t)}{z(\alpha+h, t)-z(\beta+h, t)}\right) \rho(\beta)\left[\frac{1}{z_{h}}\right]_{h}(\beta, t) d \beta \\
&+ \frac{1}{h^{2}} \int\left(\frac{z_{\beta}(\beta+2 h, t)}{z(\alpha+2 h, t)-z(\beta+2 h, t)}-\frac{z_{\beta}(\beta+h, t)}{z(\alpha+h, t)-z(\beta+h, t)}\right) \\
& \cdot \rho(\beta+h)\left[\frac{1}{z_{h}}\right]_{h}(\beta+h, t) d \beta \\
&=\frac{1}{h^{2}} \int\left(\frac{z_{\beta}(\beta, t)}{z(\alpha, t)-z(\beta, t)}-\frac{z_{\beta}(\beta+h, t)}{z(\alpha+h, t)-z(\beta+h, t)}\right) \rho(\beta)\left[\frac{1}{z_{h}}\right]_{h}(\beta, t) d \beta \\
&+\frac{1}{h^{2}} \int\left(\frac{z_{\beta}(\beta+h, t)}{z(\alpha+2 h, t)-z(\beta+h, t)}-\frac{z_{\beta}(\beta, t)}{z(\alpha+h, t)-z(\beta, t)}\right) \\
& \cdot \rho(\beta)\left[\frac{1}{z_{h}}\right]_{h}(\beta, t) d \beta .
\end{aligned}
$$

Using integration by parts and a similar calculation as in (4.9), we get

$$
\begin{aligned}
\mathrm{II}_{1}+ & \mathrm{III}_{1} \\
= & \frac{1}{h^{2}} \int_{0}^{h} \int\left(\frac{z_{\alpha}(\alpha+h+\gamma, t)}{z(\alpha+h+\gamma, t)-z(\beta+h, t)}-\frac{z_{\alpha}(\alpha+\gamma, t)}{z(\alpha+\gamma, t)-z(\beta, t)}\right) \\
& \cdot \partial_{\beta}\left\{\rho\left[\frac{1}{z_{h}}\right]_{h}\right\}(\beta, t) d \beta d \gamma \\
= & \frac{1}{h} \int_{0}^{h} \int \frac{z_{\alpha h}(\alpha+\gamma, t)}{z(\alpha+\gamma, t)-z(\beta, t)} \partial_{\beta}\left\{\rho(\beta)\left[\frac{1}{z_{h}}\right]_{h}(\beta, t)\right\} d \beta d \gamma \\
+ & \frac{1}{h^{2}} \int_{0}^{h} z_{\alpha}(\alpha+h+\gamma, t) \\
& \cdot \int\left(\frac{1}{z(\alpha+h+\gamma, t)-z(\beta+h, t)}-\frac{1}{z(\alpha+\gamma, t)-z(\beta, t)}\right) \\
= & \mathrm{V}_{1}+\partial_{\beta}\left\{\rho(\beta)\left[\frac{1}{z_{h}}\right]_{h}(\beta, t)\right\} d \beta d \gamma
\end{aligned}
$$

Now

$$
\mathrm{V}_{1}=\frac{1}{h} \int_{0}^{h} \int \frac{z_{\alpha h}(\alpha+\gamma, t)}{z(\alpha+\gamma, t)-z(\beta, t)} \partial_{\beta}\left\{\rho(\beta)\left[\frac{1}{z_{h}}\right]_{h}(\beta, t)\right\} d \beta d \gamma
$$




$$
\begin{aligned}
= & \frac{1}{h} \int_{0}^{h} \int_{\frac{z_{\alpha h}(\alpha+\gamma, t)}{z(\alpha+\gamma, t)-z(\beta, t)} \rho^{\prime}(\beta)\left[\frac{1}{z_{h}}\right]_{h}(\beta, t) d \beta d \gamma} \\
& -\frac{1}{h} \int_{0}^{h} \int \frac{z_{\alpha h}(\alpha+\gamma, t)-z_{h h}(\alpha, t)}{z(\alpha+\gamma, t)-z(\beta, t)} \rho(\beta) \frac{z_{h h}(\beta, t)}{z_{h}(\beta, t) z_{h}(\beta+h, t)} d \beta d \gamma \\
& -z_{h h}(\alpha, t) \frac{1}{h} \int_{0}^{h} \int \frac{1}{z(\alpha+\gamma, t)-z(\beta, t)} \rho(\beta) \frac{z_{h h}(\beta, t)}{z_{h}(\beta, t) z_{h}(\beta+h, t)} d \beta d \gamma \\
= & \mathrm{V}_{11}-\mathrm{V}_{12}-\mathrm{V}_{13} .
\end{aligned}
$$

Since $\rho^{\prime}(\beta)=0$ for $\beta \in \frac{3}{2} Q$ and $\beta \notin 2 Q$, we have

$$
\left|\eta(\alpha) \mathrm{V}_{11}\right| \leq \frac{c_{1}}{|Q|} \frac{1}{h} \int_{0}^{h}\left|z_{\alpha h}(\alpha+\gamma, t)\right| d \gamma
$$

here $c_{1}$ is a constant that depends on $m$.

Notice that $\mathrm{V}_{13}$ can be estimated using Theorem 2.3 and Lemma 4.2.

We now consider $\mathrm{V}_{12}$. Define for any function $f$ that

$$
C_{1}(f)(\alpha)=\int \frac{1}{z(\alpha, t)-z(\beta, t)} f(\beta) d \beta .
$$

Let

$$
g(\beta)=\rho(\beta) \frac{h z_{h h}(\beta, t)}{z_{h}(\beta, t) z_{h}(\beta+h, t)} .
$$

Since for any function $b \in \mathrm{BMO}(\mathbb{R})$ and $\beta \in \mathbb{R}$,

$$
\begin{aligned}
& \left|[b]_{h}(\beta+h)-[b]_{h}(\beta)\right| \\
& \quad \leq \frac{1}{h^{2}} \int_{\beta}^{\beta+2 h} \int_{\beta}^{\beta+2 h}|b(x)-b(y)| d x d y \leq 8\|b\|_{\text {вмо }}
\end{aligned}
$$

then

$$
\|g\|_{L^{\infty}} \leq \frac{8}{m^{2}}\left\|z_{\alpha}\right\|_{\mathrm{BMO}(a, b), \delta_{0}} .
$$

From the above notation, we have

$$
\mathrm{V}_{12}=\frac{1}{h} \int_{0}^{h} \frac{z_{\alpha h}(\alpha+\gamma, t)-z_{h h}(\alpha, t)}{h} C_{1}(g)(\alpha+\gamma) d \gamma .
$$

Notice that

$$
\int_{0}^{h}\left(z_{\alpha h}(\alpha+\gamma, t)-z_{h h}(\alpha, t)\right) d \gamma=0 .
$$

So for any constant $c$,

$$
\mathrm{V}_{12}=\frac{1}{h} \int_{0}^{h} \frac{z_{\alpha h}(\alpha+\gamma, t)-z_{h h}(\alpha, t)}{h}\left(C_{1}(g)(\alpha+\gamma)-c\right) d \gamma
$$


Because the operator $C_{1}$ is bounded from $L^{\infty}(\mathbb{R}) \rightarrow \operatorname{BMO}(\mathbb{R})$ (see $[25$, p. 49]), there exists a universal constant $c_{2}$ such that

$$
\begin{aligned}
\left|\mathrm{V}_{12}\right| \leq & \left(\frac{1}{h} \int_{0}^{h}\left|\frac{z_{\alpha h}(\alpha+\gamma, t)-z_{h h}(\alpha, t)}{h}\right|^{2} d \gamma\right)^{1 / 2} \\
& \cdot \inf _{c}\left(\frac{1}{h} \int_{0}^{h}\left|C_{1}(g)(\alpha+\gamma)-c\right|^{2} d \gamma\right)^{1 / 2} \\
\leq & c_{2}\|g\|_{L^{\infty}}\left(\frac{1}{h} \int_{0}^{h}\left|\frac{z_{\alpha h}(\alpha+\gamma, t)-z_{h h}(\alpha, t)}{h}\right|^{2} d \gamma\right)^{1 / 2} \\
\leq & \frac{8 c_{2}}{m^{2}}\left\|z_{\alpha}\right\|_{\mathrm{BMO}(a, b), \delta_{0}}\left(\frac{1}{h} \int_{0}^{h}\left|\frac{z_{\alpha h}(\alpha+\gamma, t)-z_{h h}(\alpha, t)}{h}\right|^{2} d \gamma\right)^{1 / 2} .
\end{aligned}
$$

To obtain an estimate for $\mathrm{V}_{2}$, we consider

$$
\begin{aligned}
\frac{1}{h} \int & \left(\frac{1}{z(\alpha+h, t)-z(\beta+h, t)}-\frac{1}{z(\alpha, t)-z(\beta, t)}\right) \partial_{\beta}\left\{\rho(\beta)\left[\frac{1}{z_{h}}\right]_{h}(\beta, t)\right\} d \beta \\
= & \int \frac{z_{\beta}(\beta+h, t)}{z(\alpha+h, t)-z(\beta+h, t)}\left(\frac{1}{z_{\beta}}\right)_{h}(\beta, t) \partial_{\beta}\left\{\rho(\beta)\left[\frac{1}{z_{h}}\right]_{h}(\beta, t)\right\} d \beta \\
& +\frac{1}{h} \int\left(\frac{z_{\beta}(\beta+h, t)}{z(\alpha+h, t)-z(\beta+h, t)}-\frac{z_{\beta}(\beta, t)}{z(\alpha, t)-z(\beta, t)}\right) \frac{1}{z_{\beta}(\beta, t)} \\
= & -\int \frac{z_{\beta}\left\{\rho(\beta)\left[\frac{1}{z_{h}}\right]_{h}(\beta, t)\right\} d \beta}{z(\alpha+h, t)-z(\beta+h, t)} \frac{z_{\beta h}(\beta, t)}{z_{\beta}(\beta, t) z_{\beta}(\beta+h, t)} \partial_{\beta}\left\{\rho(\beta)\left[\frac{1}{z_{h}}\right]_{h}(\beta, t)\right\} d \beta \\
+ & \int\left(\frac{z_{\beta}(\beta+h, t)}{z(\alpha+h, t)-z(\beta+h, t)}-\frac{z_{\beta}(\beta, t)}{z(\alpha, t)-z(\beta, t)}\right) \frac{1}{h}\left(\frac{1}{z_{\beta}}-\frac{1}{z_{h}}\right) \\
& \cdot \partial_{\beta}\left\{\rho(\beta)\left[\frac{1}{z_{h}}\right]_{h}(\beta, t)\right\} d \beta \\
+ & \frac{1}{h} \int_{0}^{h} \int \frac{z_{\alpha}(\alpha+\gamma, t)-z_{\beta}(\beta+\gamma, t)}{z(\alpha+\gamma, t)-z(\beta+\gamma, t)} \partial_{\beta}\left\{\frac{1}{z_{h}(\beta, t)} \partial_{\beta}\left\{\rho(\beta)\left[\frac{1}{z_{h}}\right]_{h}(\beta, t)\right\}\right\} d \beta .
\end{aligned}
$$

In the last step of (4.18), we used integration by parts.

Furthermore,

$$
\begin{aligned}
z_{\beta h}(\beta, t) \partial_{\beta}\left\{\rho(\beta)\left[\frac{1}{z_{h}}\right]_{h}(\beta, t)\right\} \\
=z_{\beta h}(\beta, t) \rho^{\prime}(\beta)\left[\frac{1}{z_{h}}\right]_{h}(\beta, t)+\left(z_{\beta h}-z_{h h}\right)(\beta, t) \rho(\beta)\left(\frac{1}{z_{h}}\right)_{h}(\beta, t) \\
\quad+z_{h h}(\beta, t) \rho(\beta)\left(\frac{1}{z_{h}}\right)_{h}(\beta, t) \\
\quad \frac{1}{h}\left(\frac{1}{z_{\beta}}-\frac{1}{z_{h}}\right) \partial_{\beta}\left\{\rho(\beta)\left[\frac{1}{z_{h}}\right]_{h}(\beta, t)\right\}
\end{aligned}
$$




$$
\begin{aligned}
= & \frac{1}{h}\left(\frac{1}{z_{\beta}}-\frac{1}{z_{h}}\right) \rho^{\prime}(\beta)\left[\frac{1}{z_{h}}\right]_{h}(\beta, t) \\
& -\frac{z_{\beta}(\beta, t)+z_{2 h}(\beta, t)-2 z_{h}(\beta, t)}{h^{2} z_{\beta}(\beta, t) z_{h}(\beta, t)} h \rho(\beta)\left(\frac{1}{z_{h}}\right)_{h}(\beta, t) \\
& +\frac{z_{h h}(\beta, t)}{2 z_{\beta}(\beta, t) z_{h}(\beta, t)} \rho(\beta)\left(\frac{1}{z_{h}}\right)_{h}(\beta, t),
\end{aligned}
$$

and

$$
\begin{aligned}
\partial_{\beta}\{ & \left.\frac{1}{z_{h}(\beta, t)} \partial_{\beta}\left\{\rho(\beta)\left[\frac{1}{z_{h}}\right]_{h}(\beta, t)\right\}\right\} \\
= & -\frac{z_{\beta h}(\beta, t)}{z_{h}^{2}(\beta, t)}\left(\rho^{\prime}(\beta)\left[\frac{1}{z_{h}}\right]_{h}(\beta, t)-\rho(\beta) \frac{z_{h h}(\beta, t)}{z_{h}(\beta, t) z_{h}(\beta+h, t)}\right) \\
& +\frac{1}{z_{h}(\beta, t)}\left(\rho^{\prime \prime}(\beta)\left[\frac{1}{z_{h}}\right]_{h}(\beta, t)-2 \rho^{\prime}(\beta) \frac{z_{h h}(\beta, t)}{z_{h}(\beta, t) z_{h}(\beta+h, t)}\right) \\
& -\rho(\beta) \frac{z_{\beta h h}(\beta, t)}{z_{h}^{2}(\beta, t) z_{h}(\beta+h, t)} \\
& +\rho(\beta) \frac{z_{h h}(\beta, t) z_{\beta h}(\beta, t) z_{h}(\beta+h, t)+z_{h h}(\beta, t) z_{\beta h}(\beta+h, t) z_{h}(\beta, t)}{z_{h}^{3}(\beta, t) z_{h}^{2}(\beta+h, t)}
\end{aligned}
$$

So $\eta(\alpha) \mathrm{V}_{2}$ can be estimated using (4.3), (4.4), Theorem 2.3, Corollary 2.4, and Theorem 2.6.

For the estimate of $\eta(\alpha) \mathrm{I}$, we notice further that

$$
\begin{aligned}
& \left(\frac{1}{z_{\beta}}\right)_{h h}(\beta, t) \\
& \quad=-\left(\frac{z_{\beta h}(\beta, t)}{z_{\beta}(\beta, t) z_{\beta}(\beta+h, t)}\right)_{h} \\
& \quad=-\frac{z_{\beta h h}(\beta, t)}{z_{\beta}(\beta+h, t) z_{\beta}(\beta+2 h, t)}+\frac{z_{\beta h}(\beta, t)\left(z_{\beta h}(\beta, t)+z_{\beta h}(\beta+h, t)\right)}{z_{\beta}(\beta, t) z_{\beta}(\beta+h, t) z_{\beta}(\beta+2 h, t)},
\end{aligned}
$$

and

(4.21) $z_{\beta h}^{2}(\beta, t)$

$$
=\frac{1}{h}\left(z_{\beta h}(\beta, t)-z_{h h}(\beta, t)\right)\left(h z_{\beta h}(\beta, t)+h z_{h h}(\beta, t)\right)+z_{h h}^{2}(\beta, t) .
$$


Summing up the calculation from (4.7) to (4.21) and applying Theorem 2.3, Corollary 2.4, Theorem 2.6, Propositions 2.7 and 2.8, and Lemma 4.3, we arrive at

$$
\begin{aligned}
\int \mid & \left.\eta(\alpha) G_{2}(\alpha, t)\right|^{2} d \alpha \\
\leq & c_{1}\left\|\ln z_{\alpha}\right\|_{\mathrm{BMO}(a, b), \delta_{0}}^{2} \\
& \cdot\left(\int_{2 Q}\left|z_{\alpha h h}(\alpha, t)\right|^{2} d \alpha+\int_{2 Q} \frac{1}{h^{5}} \int_{0}^{h}\left|k^{2} z_{\alpha k k}(\alpha, t)\right|^{2} d k d \alpha\right) \\
& +\left.\left.c_{2} \int_{2}|(\rho(\alpha)+\rho(\alpha-h)+\rho(\alpha-2 h))| z_{h h}(\alpha, t)\right|^{2}\right|^{2} d \alpha \\
& +\frac{c_{3}}{|Q|^{2}} \int_{2 Q}\left|z_{\alpha \alpha}(\alpha, t)\right|^{2} d \alpha+\frac{c_{4}}{|Q|^{3}},
\end{aligned}
$$

where $c_{1}, c_{2}, c_{3}$, and $c_{4}$ are constants independent of $h$. We can estimate

$$
\left\|[\eta, H]\left\{\rho\left(\frac{1}{z_{\alpha}}\right)_{h h}\right\}\right\|_{L^{2}(\mathbb{R})}
$$

in the same way as in Lemma 3.5:

$$
\begin{aligned}
{[\eta, H] } & \left\{\rho\left(\frac{1}{z_{\alpha}}\right)_{h h}\right\}(\alpha, t) \\
= & \frac{1}{h} \int \frac{\eta(\alpha)-\eta(\beta)}{\alpha-\beta}\left(\frac{z_{\beta h}(\beta, t)}{z_{\beta}(\beta, t) z_{\beta}(\beta+h, t)}-\frac{z_{\beta h}(\beta+h, t)}{z_{\beta}(\beta+h, t) z_{\beta}(\beta+2 h, t)}\right) \rho(\beta) d \beta \\
= & -\frac{1}{h} \int\left(\frac{\eta(\alpha)-\eta(\beta-h)}{\alpha-\beta+h}-\frac{\eta(\alpha)-\eta(\beta)}{\alpha-\beta}\right) \frac{z_{\beta h}(\beta, t)}{z_{\beta}(\beta, t) z_{\beta}(\beta+h, t)} \rho(\beta) d \beta \\
& +\int \frac{\eta(\alpha)-\eta(\beta)}{\alpha-\beta} \frac{1}{h}(\rho(\beta+h)-\rho(\beta)) \frac{z_{\beta h}(\beta+h, t)}{z_{\beta}(\beta+h, t) z_{\beta}(\beta+2 h, t)} d \beta,
\end{aligned}
$$

where

$$
\begin{aligned}
\frac{1}{h} \int & \left(\frac{\eta(\alpha)-\eta(\beta-h)}{\alpha-\beta+h}-\frac{\eta(\alpha)-\eta(\beta)}{\alpha-\beta}\right) \frac{z_{\beta h}(\beta, t)}{z_{\beta}(\beta, t) z_{\beta}(\beta+h, t)} \rho(\beta) d \beta \\
= & \frac{1}{h} \iint_{0}^{h}\left(-\frac{\eta(\alpha)-\eta(\beta-r)}{(\alpha-\beta+r)^{2}}+\frac{\eta^{\prime}(\beta-r)}{\alpha-\beta+r}\right) d r \frac{z_{\beta h}(\beta, t)}{z_{\beta}(\beta, t) z_{\beta}(\beta+h, t)} \rho(\beta) d \beta \\
= & \frac{1}{h} \iint_{0}^{h}\left(-\frac{\eta(\alpha)-\eta(\beta)}{(\alpha-\beta)^{2}}+\frac{\eta^{\prime}(\beta)}{\alpha-\beta}\right) \frac{z_{\beta h}(\beta+r, t)}{z_{\beta}(\beta+r, t) z_{\beta}(\beta+h+r, t)} d r d \beta .
\end{aligned}
$$

Therefore for some constant $c_{5}$ depending on $m$,

$$
\int\left|[\eta, H]\left\{\rho\left(\frac{1}{z_{\alpha}}\right)_{h h}\right\}(\alpha, t)\right|^{2} d \alpha \leq \frac{c_{5}}{|Q|^{2}} \int_{2 Q}\left|z_{\alpha \alpha}(\alpha, t)\right|^{2} d \alpha .
$$


We now estimate

$$
\begin{aligned}
\int \mid 2 \pi i \eta(\alpha) \bar{z}_{t h h}(\alpha, t)-\int & \left.\frac{\eta(\beta)}{\alpha-\beta}\left(\frac{1}{z_{\beta}}\right)_{h h}(\beta, t) d \beta\right|^{2} d \alpha \\
& =\pi^{2} \int\left|2 i \eta(\alpha) \bar{z}_{t h h}(\alpha, t)-H\left(\eta\left(\frac{1}{z_{\alpha}}\right)_{h h}\right)\right|^{2} d \alpha .
\end{aligned}
$$

We know

$$
\begin{aligned}
\int \mid & 2 i \eta(\alpha) \bar{z}_{t h h}(\alpha, t)-\left.H\left(\eta\left(\frac{1}{z_{\alpha}}\right)_{h h}\right)\right|^{2} d \alpha \\
= & 4 \int\left|\eta(\alpha) z_{t h h}(\alpha, t)\right|^{2} d \alpha+\int\left|H\left(\eta\left(\frac{1}{z_{\alpha}}\right)_{h h}\right)\right|^{2} d \alpha \\
& -\frac{4}{\pi} \operatorname{Im} \iint \frac{\eta(\alpha) \eta(\beta) z_{t h h}(\alpha, t)\left(1 / z_{\beta}\right)_{h h}(\beta, t)}{\alpha-\beta} d \alpha d \beta .
\end{aligned}
$$

Concerning the estimate of

$$
\int\left|H\left(\eta\left(\frac{1}{z_{\alpha}}\right)_{h h}\right)\right|^{2}(\alpha, t) d \alpha=\int\left|\eta\left(\frac{1}{z_{\alpha}}\right)_{h h}(\alpha, t)\right|^{2} d \alpha,
$$

we have the following lemma:

LEMMA 4.4 There exist constants $c_{6}, c_{7}, c_{8}$, and $c_{9}$, depending only on $m$ and $M$, such that

$$
\begin{aligned}
& \int_{Q}\left|z_{\alpha h h}(\alpha, t)\right|^{2} d \alpha \\
& \leq c_{6} \int_{\frac{9}{8} Q}\left|\left(\frac{1}{z_{\alpha}}\right)_{h h}(\alpha, t)\right|^{2} d \alpha+c_{7} \int_{\frac{9}{8} Q} \frac{1}{h^{5}} \int_{0}^{h}\left|k^{2}\left(\frac{1}{z_{\alpha}}\right)_{k k}(\alpha, t)\right|^{2} d \alpha d k \\
& +c_{8}\left\|\ln z_{\alpha}\right\|_{\mathrm{BMO}(a, b), \delta_{0}}^{2} \\
& \quad \cdot\left(\int_{\frac{9}{8} Q}\left|z_{\alpha h h}(\alpha, t)\right|^{2} d \alpha+\int_{\frac{9}{8} Q} \frac{1}{h^{5}} \int_{0}^{h}\left|k^{2} z_{\alpha k k}(\alpha, t)\right|^{2} d \alpha d k\right) \\
& +c_{9} \int_{\frac{9}{8} Q}\left|z_{h h}(\alpha, t)\right|^{4} d \alpha .
\end{aligned}
$$


PROOF: We have

$$
\begin{aligned}
\left(\frac{1}{z_{\alpha}}\right)_{h h}(\alpha, t)= & -\left(\frac{z_{\alpha h}(\alpha, t)}{z_{\alpha}(\alpha, t) z_{\alpha}(\alpha+h, t)}\right)_{h} \\
= & -\frac{z_{\alpha h h}(\alpha, t)}{z_{\alpha}(\alpha+h, t) z_{\alpha}(\alpha+2 h, t)}-\frac{h z_{\alpha h}(\alpha, t)}{z_{\alpha}(\alpha+h, t)}\left(\frac{1}{z_{\alpha}}\right)_{h h}(\alpha, t) \\
& -\frac{2 h z_{\alpha h}(\alpha, t)}{z_{\alpha}(\alpha+h, t)} \frac{1}{h}\left\{\left(\frac{1}{z_{\alpha}}\right)_{h}(\alpha, t)-\left[\left(\frac{1}{z_{\alpha}}\right)_{h}\right]_{h}(\alpha, t)\right\} \\
& -\frac{2 z_{\alpha h}(\alpha, t)}{z_{\alpha}(\alpha+h, t)}\left[\left(\frac{1}{z_{\alpha}}\right)_{h}\right]_{h}(\alpha, t),
\end{aligned}
$$

where

$$
\begin{aligned}
& \frac{z_{\alpha h}(\alpha, t)}{z_{\alpha}(\alpha+h, t)}\left[\left(\frac{1}{z_{\alpha}}\right)_{h}\right]_{h}(\alpha, t) \\
& =\frac{z_{\alpha h}(\alpha, t)-z_{h h}(\alpha, t)}{h z_{\alpha}(\alpha+h, t)}\left(\left[\frac{1}{z_{\alpha}}\right]_{h}(\alpha+h, t)-\left[\frac{1}{z_{\alpha}}\right]_{h}(\alpha, t)\right) \\
& \quad+\frac{z_{h h}(\alpha, t)}{z_{\alpha}(\alpha+h, t)}\left[\left(\frac{1}{z_{\alpha}}\right)\right]_{h}(\alpha, t) \\
& =\frac{1}{h}\left(z_{\alpha h}(\alpha, t)-z_{h h}(\alpha, t)\right) \frac{1}{z_{\alpha}(\alpha+h, t)}\left(\left[\frac{1}{z_{\alpha}}\right]_{h}(\alpha+h, t)-\left[\frac{1}{z_{\alpha}}\right]_{h}(\alpha, t)\right) \\
& \quad-\frac{h z_{h h}(\alpha, t)}{z_{\alpha}(\alpha+h, t)}\left[\frac{z_{\alpha h}(\alpha, t)-z_{h h}(\alpha, t)}{h z_{\alpha}(\alpha, t) z_{\alpha}(\alpha+h, t)}\right]_{h} \\
& \quad-\frac{z_{h h}(\alpha, t)}{z_{\alpha}(\alpha+h, t)}\left[\frac{z_{h h}(\alpha, t)}{z_{\alpha}(\alpha, t) z_{\alpha}(\alpha+h, t)}\right]_{h}
\end{aligned}
$$

Therefore Lemma 4.4 follows from (4.4), (4.16), and Propositions 2.7 and 2.8.

We now consider the term

$$
-\frac{4}{\pi} \operatorname{Im} \iint \frac{\eta(\alpha) \eta(\beta) z_{t h h}(\alpha, t)\left(1 / z_{\beta}\right)_{h h}(\beta, t)}{\alpha-\beta} d \alpha d \beta .
$$

We calculate as follows:

$$
\begin{aligned}
& -\iint \frac{\eta(\alpha) \eta(\beta) z_{t h h}(\alpha, t)\left(1 / z_{\beta}\right)_{h h}(\beta, t)}{\alpha-\beta} d \alpha d \beta \\
& =\iint \frac{\eta(\alpha) \eta(\beta)}{\alpha-\beta} z_{t h h}(\alpha, t)\left(\frac{z_{\beta h}(\beta, t)}{z_{\beta}(\beta, t) z_{\beta}(\beta+h, t)}\right)_{h} d \alpha d \beta
\end{aligned}
$$




$$
\begin{aligned}
& =\iint \frac{\eta(\alpha) \eta(\beta)}{\alpha-\beta} z_{t h h}(\alpha, t)\left(\frac{z_{\beta h}(\beta, t)}{z_{h}^{2}(\beta, t)}\right)_{h} d \alpha d \beta \\
& +\iint \frac{\eta(\alpha) \eta(\beta)}{\alpha-\beta} z_{t h h}(\alpha, t) \\
& \quad \cdot\left(z_{\beta h}(\beta, t)\left(\frac{1}{z_{\beta}(\beta, t) z_{\beta}(\beta+h, t)}-\frac{1}{z_{h}^{2}(\beta, t)}\right)\right)_{h} d \alpha d \beta \\
& =\mathrm{VI}_{1}+\mathrm{VI}_{2} .
\end{aligned}
$$

For $\mathrm{VI}_{2}$, we further decompose

$$
\begin{aligned}
& \left(z_{\beta h}(\beta, t)\left(\frac{1}{z_{\beta}(\beta, t) z_{\beta}(\beta+h, t)}-\frac{1}{z_{h}^{2}(\beta, t)}\right)\right)_{h} \\
& =z_{\beta h h}(\beta, t)\left(\frac{1}{z_{\beta}(\beta, t) z_{\beta}(\beta+h, t)}-\frac{1}{z_{h}^{2}(\beta, t)}\right) \\
& \quad+\left(z_{\beta h}(\beta+h, t)-z_{h h}(\beta+h, t)\right)\left(\frac{1}{z_{\beta}(\beta, t) z_{\beta}(\beta+h, t)}-\frac{1}{z_{h}^{2}(\beta, t)}\right)_{h} \\
& \quad+z_{h h}(\beta+h, t)\left(\frac{1}{z_{\beta}(\beta, t) z_{\beta}(\beta+h, t)}-\frac{1}{z_{h}^{2}(\beta, t)}\right)_{h} .
\end{aligned}
$$

Furthermore,

$$
\begin{aligned}
& \frac{1}{z_{\beta}(\beta, t) z_{\beta}(\beta+h, t)}-\frac{1}{z_{h}^{2}(\beta, t)} \\
&=\left(\frac{1}{z_{\beta}(\beta, t)}-\frac{1}{z_{h}(\beta, t)}\right) \frac{1}{z_{\beta}(\beta+h, t)} \\
& \quad+\frac{1}{z_{h}(\beta, t)}\left(\frac{1}{z_{\beta}(\beta+h, t)}-\frac{1}{z_{h}(\beta+h, t)}\right)-\frac{h z_{h h}(\beta, t)}{z_{h}^{2}(\beta, t) z_{h}(\beta+h, t)} \\
&=\left(\frac{1}{z_{\beta}(\beta, t)}-\frac{2}{z_{h}(\beta, t)}+\frac{1}{z_{2 h}(\beta, t)}\right) \frac{1}{z_{\beta}(\beta+h, t)} \\
&+\frac{1}{z_{h}(\beta, t)}\left(\frac{1}{z_{\beta}(\beta+h, t)}-\frac{2}{z_{h}(\beta+h, t)}+\frac{1}{z_{2 h}(\beta+h, t)}\right) \\
&+\left(\frac{1}{z_{h}(\beta, t)}-\frac{1}{z_{2 h}(\beta, t)}\right) \frac{1}{z_{\beta}(\beta+h, t)} \\
&+\frac{1}{z_{h}(\beta, t)}\left(\frac{1}{z_{h}(\beta+h, t)}-\frac{1}{z_{2 h}(\beta+h, t)}\right)-\frac{h z_{h h}(\beta, t)}{z_{h}^{2}(\beta, t) z_{h}(\beta+h, t)} .
\end{aligned}
$$


Therefore an application of Lemmas 3.4 and 4.3, a similar calculation as in (3.70), and an application of Theorem 2.6, (4.16), and Propositions 2.7 and 2.8 give that

$$
\begin{aligned}
\frac{4}{\pi}\left|\mathrm{VI}_{2}\right| \leq & \int\left|\eta(\alpha) z_{t h h}(\alpha, t)\right|^{2} d \alpha \\
+ & c_{10}\left\|\ln z_{\alpha}\right\|_{\mathrm{BMO}(a, b), \delta_{0}}^{2} \\
& \cdot\left(\int_{2 Q}\left|z_{\alpha h h}(\alpha, t)\right|^{2} d \alpha+\int_{2 Q} \frac{1}{h^{5}} \int_{0}^{h}\left|k^{2} z_{\alpha k k}(\alpha, t)\right|^{2} d \alpha d k\right) \\
+ & c_{11}\left\|\ln z_{\alpha}\right\|_{\mathrm{BMO}(a, b), \delta_{0}}^{1 / 2} \\
& \cdot\left(\int_{2 Q}\left|z_{\alpha h h}(\alpha, t)\right|^{2} d \alpha+\int_{2 Q} \frac{1}{h^{5}} \int_{0}^{h}\left|k^{2} z_{\alpha k k}(\alpha, t)\right|^{2} d \alpha d k\right) \\
+ & c_{12} \int_{\frac{3}{2} Q}\left|z_{h h}(\alpha, t)\right|^{4} d \alpha,
\end{aligned}
$$

where $c_{10}, c_{11}$, and $c_{12}$ are constants that depend on $m$ and $M$. For $\mathrm{VI}_{1}$, we have

$$
\begin{aligned}
\iint & \frac{\eta(\alpha) \eta(\beta)}{\alpha-\beta} z_{t h h}(\alpha, t)\left(\frac{z_{\beta h}(\beta, t)}{z_{h}^{2}(\beta, t)}\right)_{h} d \alpha d \beta \\
= & \iint \frac{\eta(\alpha) \eta(\beta)}{\alpha-\beta} z_{t h h}(\alpha, t)\left(\frac{z_{\beta h h}(\beta, t)}{z_{h}^{2}(\beta, t)}+z_{\beta h}(\beta+h, t)\left(\frac{1}{z_{h}^{2}}\right)_{h}(\beta, t)\right) d \alpha d \beta \\
= & \iint \frac{\eta(\alpha) \eta(\beta)}{\alpha-\beta} \frac{z_{t h h}(\alpha, t) z_{\beta h h}(\beta, t)}{z_{h}(\alpha, t) z_{h}(\beta, t)} d \alpha d \beta \\
& +\iint \frac{\eta(\alpha) \eta(\beta)}{\alpha-\beta} z_{t h h}(\alpha, t)\left(\frac{1}{z_{h}(\beta, t)}-\frac{1}{z_{h}(\alpha, t)}\right) \frac{z_{\beta h h}(\beta, t)}{z_{h}(\beta, t)} d \alpha d \beta \\
& +\iint \frac{\eta(\alpha) \eta(\beta)}{\alpha-\beta} z_{t h h}(\alpha, t)\left(z_{\beta h}(\beta+h, t)-z_{h h}(\beta+h, t)\right)\left(\frac{1}{z_{h}^{2}}\right)_{h}(\beta, t) d \alpha d \beta \\
& +\iint \frac{\eta(\alpha) \eta(\beta)}{\alpha-\beta} z_{t h h}(\alpha, t) z_{h h}(\beta+h, t)\left(\frac{1}{z_{h}^{2}}\right)_{h}(\beta, t) d \alpha d \beta,
\end{aligned}
$$

where for the term

$$
\iint \frac{\eta(\alpha) \eta(\beta)}{\alpha-\beta} \frac{z_{t h h}(\alpha, t) z_{\beta h h}(\beta, t)}{z_{h}(\alpha, t) z_{h}(\beta, t)} d \alpha d \beta
$$

we follow our calculation by decomposing

$$
\frac{z_{t h h}(\alpha, t)}{z_{h}(\alpha, t)}=\partial_{t}\left(\frac{z_{h h}(\alpha, t)}{z_{h}(\alpha, t)}\right)+\frac{z_{h h}(\alpha, t) z_{t h}(\alpha, t)}{z_{h}^{2}(\alpha, t)}
$$


and

$$
\begin{aligned}
\frac{z_{\beta h h}(\beta, t)}{z_{h}(\beta, t)}= & \partial_{\beta}\left(\frac{z_{h h}(\beta, t)}{z_{h}(\beta, t)}\right) \\
& +\frac{z_{h h}(\beta, t)\left(z_{\beta h}(\beta, t)-z_{h h}(\beta, t)\right)}{z_{h}^{2}(\beta, t)}+\frac{z_{h h}^{2}(\beta, t)}{z_{h}^{2}(\beta, t)} .
\end{aligned}
$$

We further decompose the term

$$
\frac{z_{h h}(\alpha, t) z_{t h}(\alpha, t)}{z_{h}^{2}(\alpha, t)}
$$

in (4.30) or, more precisely, the term $z_{t h}(\alpha, t)$ through the following consideration. From (1.6) we have

$$
\begin{aligned}
\bar{z}_{t h}(\alpha, t)= & \frac{1}{2 \pi i h} \int\left(\frac{1}{z(\alpha+h, t)-z(\beta+h, t)}-\frac{1}{z(\alpha, t)-z(\beta, t)}\right) \rho(\beta) d \beta \\
& +\frac{1}{2 \pi i} \int \frac{-z_{h}(\alpha, t)+z_{h}(\beta, t)}{(z(\alpha+h, t)-z(\beta+h, t))(z(\alpha, t)-z(\beta, t))}(1-\rho(\beta)) d \beta,
\end{aligned}
$$

where it is clear that for $\alpha \in \frac{5}{4} Q$,

$$
\left|\frac{1}{2 \pi i} \int \frac{-z_{h}(\alpha, t)+z_{h}(\beta, t)}{(z(\alpha+h, t)-z(\beta+h, t))(z(\alpha, t)-z(\beta, t))}(1-\rho(\beta)) d \beta\right| \leq \frac{C^{\prime}}{|Q|}
$$

for some constant $C^{\prime}$.

On the other hand,

$$
\begin{aligned}
& \frac{1}{h} \int\left(\frac{1}{z(\alpha+h, t)-z(\beta+h, t)}-\frac{1}{z(\alpha, t)-z(\beta, t)}\right) \rho(\beta) d \beta \\
&= \int \frac{z_{\beta}(\beta+h, t)}{z(\alpha+h, t)-z(\beta+h, t)}\left(\frac{1}{z_{\beta}}\right)_{h}(\beta, t) \rho(\beta) d \beta \\
&+\frac{1}{h} \int\left(\frac{z_{\beta}(\beta+h, t)}{z(\alpha+h, t)-z(\beta+h, t)}-\frac{z_{\beta}(\beta, t)}{z(\alpha, t)-z(\beta, t)}\right) \frac{1}{z_{\beta}(\beta, t)} \rho(\beta) d \beta \\
&=-\int \frac{z_{\beta}(\beta+h, t)}{z(\alpha+h, t)-z(\beta+h, t)} \frac{z_{\beta h}(\beta, t)-z_{h h}(\beta, t)+z_{h h}(\beta, t)}{z_{\beta}(\beta, t) z_{\beta}(\beta+h, t)} \rho(\beta) d \beta \\
&+\frac{1}{h} \int\left(\frac{z_{\beta}(\beta+h, t)}{z(\alpha+h, t)-z(\beta+h, t)}-\frac{z_{\beta}(\beta, t)}{z(\alpha, t)-z(\beta, t)}\right) \\
&+\frac{1}{h} \int\left(\frac{1}{z_{\beta}}-\frac{2}{z_{h}}+\frac{1}{z_{2 h}}\right)(\beta, t) \rho(\beta) d \beta \\
& \quad \cdot\left(\frac{z_{\beta}(\beta+h, t)}{z_{h}(\beta+h, t)-z(\beta+h, t)}-\frac{1}{z_{2 h}(\beta, t)}\right) \rho(\beta) d \beta \\
&+\left.\frac{1}{h} \int_{0}^{h} \int \frac{z_{\alpha}(\alpha+\gamma, t)-z_{\beta}(\beta+\gamma, t)}{z_{(\alpha+\gamma}(\alpha+t)-z(\beta+\gamma, t)}\right) \\
& \partial_{\beta}\left\{\frac{\rho(\beta)}{z_{h}(\beta, t)}\right\} d \beta d \gamma
\end{aligned}
$$


where in the last term we used integration by parts.

Furthermore,

$$
\begin{aligned}
\partial_{\beta}\left\{\frac{\rho(\beta)}{z_{h}(\beta, t)}\right\}= & \frac{\rho^{\prime}(\beta)}{z_{h}(\beta, t)}-\frac{\rho(\beta)}{z_{h}^{2}(\beta, t)}\left(z_{\beta h}(\beta, t)-z_{h h}(\beta, t)\right) \\
& -\frac{\rho(\beta)}{z_{h}^{2}(\beta, t)} z_{h h}(\beta, t) .
\end{aligned}
$$

Now

$$
\begin{aligned}
\iint & \frac{\eta(\alpha) \eta(\beta)}{\alpha-\beta} \frac{z_{t h h}(\alpha, t) z_{\beta h h}(\beta, t)}{z_{h}(\alpha, t) z_{h}(\beta, t)} d \alpha d \beta \\
= & \iint \frac{\eta(\alpha) \eta(\beta)}{\alpha-\beta} \partial_{t}\left(\frac{z_{h h}(\alpha, t)}{z_{h}(\alpha, t)}\right) \partial_{\beta}\left(\frac{z_{h h}(\beta, t)}{z_{h}(\beta, t)}\right) d \alpha d \beta \\
& +\iint \frac{\eta(\alpha) \eta(\beta)}{\alpha-\beta} \frac{z_{h h}(\alpha, t) z_{t h}(\alpha, t)}{z_{h}^{2}(\alpha, t)} \partial_{\beta}\left(\frac{z_{h h}(\beta, t)}{z_{h}(\beta, t)}\right) d \alpha d \beta \\
& +\iint \frac{\eta(\alpha) \eta(\beta)}{\alpha-\beta} \frac{z_{t h h}(\alpha, t)}{z_{h}(\alpha, t)} \frac{z_{h h}(\beta, t)\left(z_{\beta h}(\beta, t)-z_{h h}(\beta, t)\right)+z_{h h}^{2}(\beta, t)}{z_{h}^{2}(\beta, t)} d \alpha d \beta .
\end{aligned}
$$

We further calculate

$$
\begin{aligned}
\iint & \frac{\eta(\alpha) \eta(\beta)}{\alpha-\beta} \partial_{t}\left(\frac{z_{h h}(\alpha, t)}{z_{h}(\alpha, t)}\right) \partial_{\beta}\left(\frac{z_{h h}(\beta, t)}{z_{h}(\beta, t)}\right) d \alpha d \beta \\
= & \iint \frac{\eta(\alpha)}{\alpha-\beta} \partial_{t}\left(\frac{z_{h h}(\alpha, t)}{z_{h}(\alpha, t)}\right) \partial_{\beta}\left(\eta(\beta) \frac{z_{h h}(\beta, t)}{z_{h}(\beta, t)}-\eta(\alpha) \frac{z_{h h}(\alpha, t)}{z_{h}(\alpha, t)}\right) d \alpha d \beta \\
& -\iint \frac{\eta(\alpha) \eta^{\prime}(\beta)}{\alpha-\beta} \partial_{t}\left(\frac{z_{h h}(\alpha, t)}{z_{h}(\alpha, t)}\right) \frac{z_{h h}(\beta, t)}{z_{h}(\beta, t)} d \alpha d \beta \\
= & \frac{1}{4} \frac{d}{d t} \iint \frac{1}{(\alpha-\beta)^{2}}\left(\eta(\alpha) \frac{z_{h h}(\alpha, t)}{z_{h}(\alpha, t)}-\eta(\beta) \frac{z_{h h}(\beta, t)}{z_{h}(\beta, t)}\right)^{2} d \alpha d \beta \\
& -\int \eta(\alpha) \partial_{t}\left(\frac{z_{h h}(\alpha, t)}{z_{h}(\alpha, t)}\right) \int \frac{\eta^{\prime}(\beta)}{\alpha-\beta} \frac{z_{h h}(\beta, t)}{z_{h}(\beta, t)} d \beta d \alpha .
\end{aligned}
$$

Summing up the calculation from (4.26) to (4.35), we obtain that for any $\epsilon>0$,

$$
\begin{aligned}
\frac{4}{\pi} \operatorname{Im} \iint & \frac{\eta(\alpha) \eta(\beta) z_{t h h}(\alpha, t)\left(1 / z_{\beta}\right)_{h h}(\beta, t)}{\alpha-\beta} d \alpha d \beta \\
\leq & -\frac{1}{\pi} \frac{d}{d t} \operatorname{Im} \iint \frac{1}{(\alpha-\beta)^{2}}\left(\eta(\alpha) \frac{z_{h h}(\alpha, t)}{z_{h}(\alpha, t)}-\eta(\beta) \frac{z_{h h}(\beta, t)}{z_{h}(\beta, t)}\right)^{2} d \alpha d \beta \\
& +3 \int\left|\eta(\alpha) z_{t h h}(\alpha, t)\right|^{2} d \alpha+\epsilon \int\left|\eta(\alpha) z_{\alpha h h}(\alpha, t)\right|^{2} d \alpha \\
+ & c_{13}\left\|\ln z_{\alpha}\right\|_{\mathrm{BMO}(a, b), \delta_{0}}^{2} \\
& \cdot\left(\int_{2 Q}\left|z_{\alpha h h}(\alpha, t)\right|^{2} d \alpha+\iint_{2 Q}^{h} \frac{1}{h^{5}}\left|k^{2} z_{\alpha k k}(\alpha, t)\right|^{2} d k d \alpha\right)
\end{aligned}
$$




$$
\begin{aligned}
& +c_{13}\left\|\ln z_{\alpha}\right\|_{\mathrm{BMO}(a, b), \delta_{0}}^{1 / 2} \\
& \cdot\left(\int_{2 Q}\left|z_{\alpha h h}(\alpha, t)\right|^{2} d \alpha+\int_{2 Q} \int_{0}^{h} \frac{1}{h^{5}}\left|k^{2} z_{\alpha k k}(\alpha, t)\right|^{2} d k d \alpha\right) \\
& +c_{14}\left(\frac{1}{\epsilon}+1\right)\left(\frac{1}{|Q|^{2}} \int_{2 Q}\left|z_{\alpha \alpha}(\alpha, t)\right|^{2} d \alpha+\int\left|\rho(\alpha) z_{h h}(\alpha, t)\right|^{4} d \alpha\right),
\end{aligned}
$$

where $c_{13}$ and $c_{14}$ are constants independent of $\epsilon$ and $h$.

Now from (4.7), we know that

$$
\begin{aligned}
\int \mid 2 i \eta(\alpha) \bar{z}_{t h h}(\alpha, t)-H & \left.\left(\eta\left(\frac{1}{z_{\alpha}}\right)_{h h}\right)\right|^{2} d \alpha \\
& =\frac{1}{\pi^{2}} \int\left|[\eta, H]\left\{\rho\left(\frac{1}{z_{\alpha}}\right)_{h h}\right\}+\eta(\alpha) G_{2}(\alpha, t)\right|^{2} d \alpha .
\end{aligned}
$$

Let $\rho_{1} \in C_{0}^{\infty}(\mathbb{R})$ be such that $\rho_{1}(\alpha)=1$ for $\alpha \in\left(\frac{15}{8}+\frac{1}{12}\right) Q, \rho_{1}(\alpha)=0$ for $\alpha \notin 2 Q$, and $\left|\rho_{1}^{\prime}(\alpha)\right| \leq C /|Q|$ for some constant $C$. Summing up the estimates (4.22), (4.23), and (4.36), we conclude that there exist constants $c_{15}, c_{16}, c_{17}$, and $c_{18}$ such that for any $0<|h|<\frac{1}{24}|Q|$ and $\epsilon>0$,

$$
\begin{aligned}
& \int\left|\eta(\alpha) z_{t h h}(\alpha, t)\right|^{2} d \alpha+\int\left|\eta(\alpha)\left(\frac{1}{z_{\alpha}}\right)_{h h}(\alpha, t)\right|^{2} d \alpha \\
& \leq- \frac{1}{\pi} \operatorname{Im} \frac{d}{d t} \iint \frac{1}{(\alpha-\beta)^{2}}\left(\eta(\alpha) \frac{z_{h h}(\alpha, t)}{z_{h}(\alpha, t)}-\eta(\beta) \frac{z_{h h}(\beta, t)}{z_{h}(\beta, t)}\right)^{2} d \alpha d \beta \\
&+ \epsilon \int\left|\eta(\alpha) z_{\alpha h h}(\alpha, t)\right|^{2} d \alpha d h \\
&+ c_{15}\left\|\ln z_{\alpha}\right\|_{\mathrm{BMO}(a, b), \delta_{0}}^{2} \\
& \cdot\left(\int_{2 Q}\left|z_{\alpha h h}(\alpha, t)\right|^{2} d \alpha d h+\frac{1}{h^{5}} \iint_{2 Q}^{h}\left|k^{2} z_{\alpha k k}(\alpha, t)\right|^{2} d k d \alpha\right) \\
&+ c_{16}\left\|\ln z_{\alpha}\right\|_{\mathrm{BMO}(a, b), \delta_{0}}^{1 / 2} \\
& \cdot\left(\int_{2 Q}\left|z_{\alpha h h}(\alpha, t)\right|^{2} d \alpha d h+\frac{1}{h^{5}} \int_{2 Q} \int_{0}^{h}\left|k^{2} z_{\alpha k k}(\alpha, t)\right|^{2} d k d \alpha\right) \\
&+ c_{17}\left(\frac{1}{\epsilon}+1\right)\left(\frac{1}{|Q|^{2}} \int_{2 Q}\left|z_{\alpha \alpha \alpha}(\alpha, t)\right|^{2} d \alpha+\int\left|\rho_{1}(\alpha) z_{h h}(\alpha, t)\right|^{4} d \alpha\right) \\
&+ \frac{c_{18}}{|Q|^{3}} .
\end{aligned}
$$


On the other hand, using (4.32) and (4.33), it is clear that there exists a constant $C_{2}^{\prime}$ such that for all $|h|>\frac{1}{24}|Q|$,

$$
\int\left|\eta(\alpha) z_{t h h}(\alpha, t)\right|^{2} d \alpha+\int\left|\eta(\alpha)\left(\frac{1}{z_{\alpha}}\right)_{h h}(\alpha, t)\right|^{2} d \alpha \leq \frac{C_{2}^{\prime}}{|Q|^{3}} .
$$

Therefore with a constant different from $c_{18}$, estimate (4.37) holds for all $h \neq 0$.

We also know that for any set $S$ and function $f(\alpha, h)$,

$$
\int_{0}^{l} h \iint_{S}^{h} \frac{1}{h^{5}} k^{4} f(\alpha, k) d k d \alpha d h=\frac{1}{3} \int_{0}^{l} \int_{S} k f(\alpha, k)\left(1-\frac{k^{3}}{l^{3}}\right) d \alpha d k .
$$

Multiply both sides of (4.37) by $h$; then integrate with respect to $h$ from 0 to $l$. Using (4.25) and (4.39), we conclude that there exist constants $v>0, c_{1}^{\prime}, c_{2}^{\prime}$, and $c_{3}^{\prime}$ such that for any $l \neq 0, \epsilon>0$,

$$
\begin{aligned}
& \frac{1}{l^{2}} \int_{0}^{l} h \int\left|\eta(\alpha) z_{t h h}(\alpha, t)\right|^{2} d \alpha d h+v \frac{1}{l^{2}} \int_{0}^{l} h \int_{Q}\left|z_{\alpha h h}(\alpha, t)\right|^{2} d \alpha d h \\
& \leq-\frac{1}{\pi l^{2}} \operatorname{Im} \frac{d}{d t} \int_{0}^{l} h \iint_{\frac{1}{(\alpha-\beta)^{2}}}\left(\eta(\alpha) \frac{z_{h h}(\alpha, t)}{z_{h}(\alpha, t)}-\eta(\beta) \frac{z_{h h}(\beta, t)}{z_{h}(\beta, t)}\right)^{2} d \alpha d \beta d h \\
& \quad+\epsilon \frac{1}{l^{2}} \int_{0}^{l} h \int_{2 Q}\left|z_{\alpha h h}(\alpha, t)\right|^{2} d \alpha d h \\
& \quad+c_{1}^{\prime}\left(\left\|\ln z_{\alpha}\right\|_{\mathrm{BMO}(a, b), \delta_{0}}^{2}+\left\|\ln z_{\alpha}\right\|_{\mathrm{BMO}(a, b), \delta_{0}}^{1 / 2}\right) \frac{1}{l^{2}} \int_{0}^{l} h \int_{2 Q}\left|z_{\alpha h h}(\alpha, t)\right|^{2} d \alpha d h \\
& \quad+c_{2}^{\prime}\left(\frac{1}{\epsilon}+1\right)\left(\frac{1}{|Q|^{2}} \int_{2 Q}\left|z_{\alpha \alpha}(\alpha, t)\right|^{2} d \alpha+\frac{1}{l^{2}} \int_{0}^{l} h \int\left|\rho_{1}(\alpha) z_{h h}(\alpha, t)\right|^{4} d \alpha d h\right) \\
& \quad+\frac{c_{3}^{\prime}}{|Q|^{3}} .
\end{aligned}
$$

\subsection{Interior $H^{2}$ Regularity of $z_{\alpha}$}

In the following, we take $0<|l|<\frac{1}{4} \delta_{1}$. Therefore for any $\alpha \in 2 Q \subset[c, d] \subset$ $(a, b)$ and $|h| \leq|l|<\frac{1}{4} \delta_{1}$, we have $\alpha+h, \alpha+2 h \in\left[c+\frac{1}{2} \delta_{1}, d-\frac{1}{2} \delta_{1}\right] \subset(a, b)$. Let $\left[T_{1}, T_{2}\right] \subset(0, T)$ be an arbitrary interval, $\delta(t)=\left(t-T_{1}\right)^{2}\left(t-T_{2}\right)^{2}$ for $t \in\left[T_{1}, T_{2}\right]$, and $\delta(t)=0$ for $t \notin\left[T_{1}, T_{2}\right]$. From (4.40), using integration by parts, we get

$$
\begin{aligned}
\frac{1}{l^{2}} \int_{0}^{l} h \iint \delta(t)\left|\eta(\alpha) z_{t h h}(\alpha, t)\right|^{2} d \alpha d t d h+v \frac{1}{l^{2}} \int_{0}^{l} h \int \delta(t) \int_{Q}\left|z_{\alpha h h}(\alpha, t)\right|^{2} d \alpha d t d h \\
\leq \frac{1}{\pi l^{2}} \operatorname{Im} \int_{0}^{l} h \int \delta^{\prime}(t) \iint_{\frac{1}{(\alpha-\beta)^{2}}}\left(\eta(\alpha) \frac{z_{h h}(\alpha, t)}{z_{h}(\alpha, t)}-\eta(\beta) \frac{z_{h h}(\beta, t)}{z_{h}(\beta, t)}\right)^{2} d \alpha d \beta d t d h \\
\quad+\frac{\epsilon}{l^{2}} \int_{0}^{l} h \int \delta(t) \int_{2}\left|z_{\alpha h h}(\alpha, t)\right|^{2} d \alpha d t d h
\end{aligned}
$$




$$
\begin{aligned}
& +c_{1}^{\prime}\left(\left\|\ln z_{\alpha}\right\|_{\mathrm{BMO}(a, b), \delta_{0}}^{2}+\left\|\ln z_{\alpha}\right\|_{\mathrm{BMO}(a, b), \delta_{0}}^{1 / 2}\right) \\
& +\frac{1}{l^{2}} \int_{0}^{l} h \int \delta(t) \int_{2 Q}\left|z_{\alpha h h}(\alpha, t)\right|^{2} d \alpha d t d h \\
& +c_{2}^{\prime}\left(\frac{1}{\epsilon}+1\right) \frac{1}{|Q|^{2}} \int_{2 Q} \delta(t) \int_{2 Q}\left|z_{\alpha \alpha}(\alpha, t)\right|^{2} d \alpha d t \\
& +c_{2}^{\prime}\left(\frac{1}{\epsilon}+1\right) \frac{1}{l^{2}} \int_{0}^{l} h \int \delta(t) \int\left|\rho_{1}(\alpha) z_{h h}(\alpha, t)\right|^{4} d \alpha d t d h+\frac{c_{3}^{\prime}}{|Q|^{3}} \int \delta(t) d t .
\end{aligned}
$$

Since $\left|\delta^{\prime}(t)\right|^{2} \leq 4\left|T_{1}-T_{2}\right|^{2} \delta(t)$ for all $t$, we have from integration by parts that for any $0<\epsilon<1$,

$$
\begin{aligned}
\mid \frac{1}{\pi l^{2}} & \operatorname{Im} \int_{0}^{l} h \int \delta^{\prime}(t) \iint \frac{1}{(\alpha-\beta)^{2}}\left(\eta(\alpha) \frac{z_{h h}(\alpha, t)}{z_{h}(\alpha, t)}-\eta(\beta) \frac{z_{h h}(\beta, t)}{z_{h}(\beta, t)}\right)^{2} d \alpha d \beta d t d h \mid \\
\leq & \left|\frac{2}{\pi l^{2}} \int_{0}^{l} h \int \delta^{\prime}(t) \iint \frac{\eta(\alpha) \eta^{\prime}(\beta)}{\alpha-\beta} \frac{z_{h h}(\alpha, t)}{z_{h}(\alpha, t)} \frac{z_{h h}(\beta, t)}{z_{h}(\beta, t)} d \alpha d \beta d t d h\right| \\
& +\left|\frac{2}{\pi l^{2}} \int_{0}^{l} h \int \delta^{\prime}(t) \iint \frac{\eta(\alpha) \eta(\beta)}{\alpha-\beta} \frac{z_{h h}(\alpha, t)}{z_{h}(\alpha, t)} \partial_{\beta}\left\{\frac{z_{h h}(\beta, t)}{z_{h}(\beta, t)}\right\} d \alpha d \beta d t d h\right| \\
\leq & \frac{4}{\pi l^{2}} \int_{0}^{l} h \iint \delta(t)\left|\eta(\alpha) z_{h h}^{2}(\alpha, t)\right|^{2} d \alpha d t d h \\
& +c_{19}\left(\frac{1}{|Q|}+\frac{1}{\epsilon}\right) \int_{T_{1}}^{T_{2}} \int_{c+\frac{1}{2} \delta_{1}}^{d-\frac{1}{2} \delta_{1}}\left|z_{\alpha \alpha}(\alpha, t)\right|^{2} d \alpha d t \\
& +\frac{\epsilon}{l^{2}} \int_{0}^{l} h \int \delta(t) \int_{2 Q}\left|z_{\alpha h h}(\alpha, t)\right|^{2} d \alpha d t d h,
\end{aligned}
$$

where $c_{19}$ is a constant depending on $M, m, T_{1}$, and $T_{2}$. We now apply Lemma 4.2 to (4.41) and (4.42). We obtain that for any $0<|l|<\frac{1}{4} \delta_{1}, 0<\epsilon<1$,

$$
\begin{aligned}
& \frac{1}{l^{2}} \int_{0}^{l} h \int \delta(t) \int_{Q}\left|z_{t h h}(\alpha, t)\right|^{2} d \alpha d t d h+v \frac{1}{l^{2}} \int_{0}^{l} h \int \delta(t) \int_{Q}\left|z_{\alpha h h}(\alpha, t)\right|^{2} d \alpha d t d h \\
& \leq \frac{2 \epsilon}{l^{2}} \int_{0}^{l} h \int_{2 Q} \delta(t) \int_{Q}\left|z_{\alpha h h}(\alpha, t)\right|^{2} d \alpha d t d h \\
& \quad+c_{1}^{\prime} \sup _{[0, T]}\left(\left\|\ln z_{\alpha}\right\|_{*, \delta_{0}}^{2}+\left\|\ln z_{\alpha}\right\|_{*, \delta_{0}}^{1 / 2}\right) \frac{1}{l^{2}} \int_{0}^{l} h \int \delta(t) \int_{2 Q}\left|z_{\alpha h h}(\alpha, t)\right|^{2} d \alpha d t d h \\
& +c_{4}^{\prime}\left(\frac{1}{\epsilon}+1\right)\left(\int_{T_{1}}^{T_{2}} \int_{c+\frac{1}{2} \delta_{1}}^{d-\frac{1}{2} \delta_{1}}\left|z_{\alpha \alpha}(\alpha, t)\right|^{2} d \alpha d t\right) \\
& \quad \cdot \frac{1}{l^{2}} \int_{0}^{l} h \int \delta(t) \int_{2 Q}\left|z_{\alpha h h}(\alpha, t)\right|^{2} d \alpha d t d h
\end{aligned}
$$




$$
\begin{aligned}
& +c_{4}^{\prime}\left(\frac{1}{\epsilon}+1\right)\left(\int_{T_{1}}^{T_{2}} \int_{c+\frac{1}{2} \delta_{1}}^{d-\frac{1}{2} \delta_{1}}\left|z_{\alpha \alpha}(\alpha, t)\right|^{2} d \alpha d t\right) \\
& +\frac{1}{l^{2}} \int_{0}^{l} h \int \delta(t) \int_{2 Q}\left|z_{t h h}(\alpha, t)\right|^{2} d \alpha d t d h \\
& +c_{5}^{\prime}\left(\left(\frac{1}{\epsilon}+1\right) \frac{1}{|Q|^{2}}+\frac{1}{\epsilon}+\frac{1}{|Q|}\right) \int_{T_{1}}^{T_{2}} \int_{c+\frac{1}{2} \delta_{1}}^{d-\frac{1}{2} \delta_{1}}\left|z_{\alpha \alpha}(\alpha, t)\right|^{2} d \alpha d t \\
& +c_{6}^{\prime}\left(\frac{1}{\epsilon}+1\right)\left(\int_{T_{1}}^{T_{2}} \int_{c+\frac{1}{2} \delta_{1}}^{d-\frac{1}{2} \delta_{1}}\left|z_{\alpha \alpha}(\alpha, t)\right|^{2} d \alpha d t\right)^{2}+\frac{c_{7}^{\prime}}{|Q|^{3}} .
\end{aligned}
$$

where $v>0, c_{1}^{\prime}$, and $c_{4}^{\prime}$ are constants depending on $m$ and $M$ only, and $c_{5}^{\prime}, c_{6}^{\prime}$, and $c_{7}^{\prime}$ are constants depending on $M, m, T_{1}$, and $T_{2}$. We want to apply the following result to (4.43):

LEMMA 4.5 Let $I \subset \mathbb{R}$ be a finite-length interval. Assume that $f, g \in L^{1}(I)$, $f, g \geq 0$, satisfy that for any interval $2 Q \subset I$,

$$
\begin{aligned}
\int_{Q} f(\alpha) d \alpha & +v \int_{Q} g(\alpha) d \alpha \\
& \leq \frac{1}{36} \int_{2 Q} f(\alpha) d \alpha+\frac{v}{36} \int_{2 Q} g(\alpha) d \alpha+C_{1}+\frac{C_{2}}{|Q|}+\frac{C_{3}}{|Q|^{2}}+\frac{C_{4}}{|Q|^{3}},
\end{aligned}
$$

where $C_{1}, C_{2}, C_{3}$, and $C_{4}$ are constants. Then

$$
\begin{aligned}
& \int_{I} \operatorname{dist}^{4}(\alpha, \partial I) f(\alpha) d \alpha+v \int_{I} \operatorname{dist}^{4}(\alpha, \partial I) g(\alpha) d \alpha \\
& \quad \leq 32 C_{1}|I|^{4}+32 C_{2}|I|^{3}+32 C_{3}|I|^{2}+32 C_{4}|I| .
\end{aligned}
$$

Here $\operatorname{dist}(\alpha, \partial I)$ is the distance from $\alpha$ to the boundary $\partial I$ of $I$.

The proof of Lemma 4.5 is a small modification of that of Proposition 3.2 and so we omit it.

We first fix $\epsilon=v / 288$. From Proposition 3.3, we know $z_{\alpha} \in H_{\mathrm{loc}}^{1}((a, b) \times$ $(0, T))$. Therefore, for small enough $T_{2}-T_{1}$, we have that

$$
c_{4}^{\prime}\left(\frac{1}{\epsilon}+1\right)\left(\int_{T_{1}}^{T_{2}} \int_{c+\frac{1}{2} \delta_{1}}^{d-\frac{1}{2} \delta_{1}}\left|z_{\alpha \alpha}(\alpha, t)\right|^{2} d \alpha d t\right) \leq \min \left\{\frac{v}{144}, \frac{1}{36}\right\} .
$$

We take the interval $\left[T_{1}, T_{2}\right]$ small enough so that (4.44) holds. We now take

$$
c_{1}(m, M)=\min \left\{c(m, M),\left(\frac{v}{144 c_{1}^{\prime}}\right)^{2},\left(\frac{v}{144 c_{1}^{\prime}}\right)^{1 / 2}\right\}
$$


where $c(m, M)$ is as defined in (3.34). Therefore for $\sup _{[0, T]}\left\|\ln z_{\alpha}(\cdot, t)\right\|_{*, \delta_{0}} \leq$ $c_{1}(m, M)$, we have

$$
\begin{aligned}
\frac{1}{l^{2}} \int_{0}^{l} h \int \delta(t) \int_{Q}\left|z_{t h h}(\alpha, t)\right|^{2} d \alpha d t d h \\
+v \frac{1}{l^{2}} \int_{0}^{l} h \int \delta(t) \int_{Q}\left|z_{\alpha h h}(\alpha, t)\right|^{2} d \alpha d t d h \\
\leq \frac{1}{36 l^{2}} \int_{0}^{l} h \int \delta(t) \int_{2 Q}\left|z_{t h h}(\alpha, t)\right|^{2} d \alpha d t d h \\
\quad+\frac{v}{36 l^{2}} \int_{0}^{l} h \int \delta(t) \int_{2 Q}\left|z_{\alpha h h}(\alpha, t)\right|^{2} d \alpha d t d h \\
\quad+c_{5}^{\prime}\left(\left(\frac{288}{v}+1\right) \frac{1}{|Q|^{2}}+\frac{288}{v}+\frac{1}{|Q|}\right) \int_{T_{1}}^{T_{2}} \int_{c+\frac{1}{2} \delta_{1}}^{d-\frac{1}{2} \delta_{1}}\left|z_{\alpha \alpha}(\alpha, t)\right|^{2} d \alpha d t \\
\quad+c_{6}^{\prime}\left(\frac{288}{v}+1\right)\left(\int_{T_{1}}^{T_{2}} \int_{c+\frac{1}{2} \delta_{1}}^{d-\frac{1}{2} \delta_{1}}\left|z_{\alpha \alpha}(\alpha, t)\right|^{2} d \alpha d t\right)^{2}+\frac{c_{7}^{\prime}}{|Q|^{3}} .
\end{aligned}
$$

Notice that (4.46) holds for any interval $2 Q \subset[c, d]$, with $|2 Q| \leq \frac{1}{2} \delta_{1}$.

Now let $I \subset[c, d] \subset(a, b)$ be any closed interval that satisfies $|I| \leq \frac{1}{2} \delta_{1}$. Notice that from Proposition 3.3 and a simply application of the Fourier transform and Plancherel's theorem, we have for any $0<|l|<\frac{1}{4} \delta_{1}$,

$$
\begin{aligned}
\frac{1}{l^{2}} \int_{0}^{l} h \int \delta(t) \int_{I}\left|z_{t h h}(\alpha, t)\right|^{2} d \alpha d t d h & \\
& +\frac{1}{l^{2}} \int_{0}^{l} h \int \delta(t) \int_{I}\left|z_{\alpha h h}(\alpha, t)\right|^{2} d \alpha d t d h<\infty .
\end{aligned}
$$

We apply Lemma 4.5 to (4.46). We get that for any $0<|l|<\frac{1}{4} \delta_{1}$,

$$
\begin{aligned}
& \frac{1}{l^{2}} \int_{0}^{l} h \int \delta(t) \int_{I} \operatorname{dist}^{4}(\alpha, \partial I)\left|z_{t h h}(\alpha, t)\right|^{2} d \alpha d t d h \\
& \quad+v \frac{1}{l^{2}} \int_{0}^{l} h \int \delta(t) \int_{I} \operatorname{dist}^{4}(\alpha, \partial I)\left|z_{\alpha h h}(\alpha, t)\right|^{2} d \alpha d t d h \leq K,
\end{aligned}
$$

where $K$ is a constant depending on $m, M, T_{1}, T_{2},|I|$, and

$$
\int_{T_{1}}^{T_{2}} \int_{c+\frac{1}{2} \delta_{1}}^{d-\frac{1}{2} \delta_{1}}\left|z_{\alpha \alpha}(\alpha, t)\right|^{2} d \alpha d t
$$


and most importantly, $K$ is independent of $l$. Let $l \rightarrow 0$. Since $[c, d] \subset(a, b)$ is arbitrary and $\left(T_{1}, T_{2}\right) \subset(0, T)$ is an arbitrary small enough interval, we conclude that

$$
z_{\alpha \alpha} \in H_{\mathrm{loc}}^{1}((a, b) \times(0, T)) .
$$

The fact that $z_{\alpha} \in C((a, b) \times(0, T))$ directly follows from a simple calculation involving Sobolev embedding. We obtain $z_{\alpha} \in H_{\mathrm{loc}}^{2}((a, b) \times(0, T))$ by taking the derivative $\partial_{t} \partial_{\alpha}$ of both sides of (1.6).

\section{2 $H^{3 / 2}$ Compatibility of the Initial Data}

We now prove the second part of Proposition 4.1. We assume in addition that $\operatorname{Im}\left((1+i) \ln z_{\alpha}\right)(\cdot, 0) \in H_{\mathrm{loc}}^{3 / 2}(a, b)$ throughout the rest of this section. Take $\zeta(t)=$ $\left(T_{1}-t\right)^{2}$ for $t \in\left[0, T_{1}\right]$ and $\zeta(t)=0$ for $t \notin\left[0, T_{1}\right]$. Instead of $\delta(t)$, we multiply (4.40) by $\zeta(t)$, integrate with respect to $t$, and go through similar calculations to those in (4.41) through (4.46). We have for $T_{1}>0$ small enough, and for the same $c_{1}(m, M)$ defined in (4.45), that

$$
\begin{aligned}
\frac{1}{l^{2}} \int_{0}^{l} h \int \zeta(t) \int_{Q}\left|z_{t h h}(\alpha, t)\right|^{2} d \alpha d t d h \\
+v \frac{1}{l^{2}} \int_{0}^{l} h \int \zeta(t) \int_{Q}\left|z_{\alpha h h}(\alpha, t)\right|^{2} d \alpha d t d h \\
\leq \frac{T_{1}^{2}}{\pi l^{2}} \operatorname{Im} \int_{0}^{l} h \iint_{\frac{1}{(\alpha-\beta)^{2}}\left(\eta(\alpha) \frac{z_{h h}(\alpha, 0)}{z_{h}(\alpha, 0)}-\eta(\beta) \frac{z_{h h}(\beta, 0)}{z_{h}(\beta, 0)}\right)^{2} d \alpha d \beta d h} \\
\quad+\frac{1}{36 l^{2}} \int_{0}^{l} h \int \zeta(t) \int\left|z_{t h h}(\alpha, t)\right|^{2} d \alpha d t d h \\
\quad+\frac{e}{36 l^{2}} \int_{0}^{l} h \int \zeta(t) \int_{2 Q}\left|z_{\alpha h h}(\alpha, t)\right|^{2} d \alpha d t d h \\
\quad+c_{1}^{\prime \prime}\left(\left(\frac{288}{v}+1\right) \frac{1}{|Q|^{2}}+\frac{288}{v}+\frac{1}{|Q|}\right) \int_{0}^{T_{1}} \int_{c+\frac{1}{2} \delta_{1}}^{d-\frac{1}{2} \delta_{1}}\left|z_{\alpha \alpha}(\alpha, t)\right|^{2} d \alpha d t \\
\quad+c_{2}^{\prime \prime}\left(\frac{288}{v}+1\right)\left(\int_{0}^{T_{1}} \int_{c+\frac{1}{2} \delta_{1}}^{d-\frac{1}{2} \delta_{1}}\left|z_{\alpha \alpha}(\alpha, t)\right|^{2} d \alpha d t\right)^{2}+\frac{c_{3}^{\prime \prime}}{|Q|^{3}},
\end{aligned}
$$

where $c_{1}^{\prime \prime}, c_{2}^{\prime \prime}$, and $c_{3}^{\prime \prime}$ are constants depending on $m, M$, and $T_{1}$.

From here we want to use arguments similar to that in Lemma 4.5 or Proposition 3.2. The only difference between (4.48) and the assumption of Lemma 4.5 is that (4.48) contains an extra term involving the initial data. We explain how to handle this term while going through the argument as that in Lemma 4.5 (which is similar to the argument of Proposition 3.2). 
Let $I_{i, k}, i=1,2, k=1,2, \ldots$, be the subintervals of $I=[p, q]$ as defined in the proof of Proposition 3.2:

$$
\begin{gathered}
\left|I_{1, k}\right|=\operatorname{dist}\left(p, I_{1, k}\right)=\frac{1}{2^{k+1}}|I|, \quad\left|I_{2, k}\right|=\operatorname{dist}\left(q, I_{2, k}\right)=\frac{1}{2^{k+1}}|I|, \\
\quad \text { for } k=1,2, \ldots
\end{gathered}
$$

and let $\eta_{i, k}$ be the function $\eta$ corresponding to the intervals $I_{i, k}$ as defined in (4.6) such that $\left|I_{i, k}\right|\left\|\eta_{i, k}^{\prime}\right\|_{\infty} \leq C$ and $\left|I_{i, k}\right|^{2}\left\|\eta_{i, k}^{\prime \prime}\right\|_{\infty} \leq C$ for some constant $C$ independent of $i$ and $k$. Define

$$
\vartheta^{2}(\alpha)=\sum_{i, k}\left|I_{i, k}\right|^{4} \eta_{i, k}^{2}(\alpha)
$$

and $\vartheta(\alpha) \geq 0$. We have $\vartheta \in C^{1,1}(\mathbb{R})$, supp $\vartheta \subset I$, and for $\alpha \in I$,

$$
\begin{aligned}
\frac{1}{16} \operatorname{dist}^{4}(\alpha, \partial I) \leq \sum_{i, k}\left|I_{i, k}\right|^{4} \chi_{I_{i, k}} \leq \vartheta^{2}(\alpha) & \leq \sum_{i, k}\left|I_{i, k}\right|^{4} \chi_{2 I_{i, k}} \\
& \leq 18 \operatorname{dist}^{4}(\alpha, \partial I) .
\end{aligned}
$$

Moreover, $2 \vartheta\left|\vartheta^{\prime}\right|=\left.2\left|\sum_{i, k}\right| I_{i, k}\right|^{4} \eta_{i, k} \eta_{i, k}^{\prime}|\leq 2 C| \sum_{i, k}\left|I_{i, k}\right|^{3} \eta_{i, k}$, so there is a constant $C^{\prime}$ such that

$$
\left|\vartheta^{\prime}(\alpha)\right| \leq C^{\prime} \sqrt{\vartheta(\alpha)}
$$

We note that for any function $f \in C^{\infty}(\mathbb{R})$,

$$
\begin{aligned}
& \sum_{i, k}\left|I_{i, k}\right|^{4} \iint \frac{\left(\eta_{i, k}(\alpha) f(\alpha)-\eta_{i, k}(\beta) f(\beta)\right)^{2}}{(\alpha-\beta)^{2}} d \alpha d \beta \\
& =\iint \frac{(\vartheta(\alpha) f(\alpha)-\vartheta(\beta) f(\beta))^{2}}{(\alpha-\beta)^{2}} d \alpha d \beta \\
& \quad+2 \iint \frac{\vartheta(\alpha) \vartheta(\beta)-\sum_{i, k}\left|I_{i, k}\right|^{4} \eta_{i, k}(\alpha) \eta_{i, k}(\beta)}{(\alpha-\beta)^{2}} f(\alpha) f(\beta) d \alpha d \beta .
\end{aligned}
$$

Furthermore,

$$
\begin{aligned}
2 \sum_{i, k}\left|I_{i, k}\right|^{4} \frac{\eta_{i, k}(\alpha) \eta_{i, k}(\beta)}{\sqrt{\vartheta(\alpha) \vartheta(\beta)}} \leq 2 \sqrt{\vartheta(\alpha) \vartheta(\beta)} & \leq \vartheta(\alpha)+\vartheta(\beta) \\
& =\sum_{i, k}\left|I_{i, k}\right|^{4}\left(\frac{\eta_{i, k}^{2}(\alpha)}{\vartheta(\alpha)}+\frac{\eta_{i, k}^{2}(\beta)}{\vartheta(\beta)}\right) .
\end{aligned}
$$


Therefore there is a constant $C_{1}$ such that

$$
\begin{aligned}
& 2\left|\iint \frac{\vartheta(\alpha) \vartheta(\beta)-\sum_{i, k}\left|I_{i, k}\right|^{4} \eta_{i, k}(\alpha) \eta_{i, k}(\beta)}{(\alpha-\beta)^{2}} f(\alpha) f(\beta) d \alpha d \beta\right| \\
& \quad \leq \sum_{i, k}\left|I_{i, k}\right|^{4} \iint \frac{1}{(\alpha-\beta)^{2}}\left(\frac{\eta_{i, k}(\alpha)}{\sqrt{\vartheta(\alpha)}}-\frac{\eta_{i, k}(\beta)}{\sqrt{\vartheta(\beta)}}\right)^{2}|f(\alpha) \sqrt{\vartheta(\alpha)} f(\beta) \sqrt{\vartheta(\beta)}| d \alpha d \beta \\
& \quad \leq C_{1} \sum_{i, k}\left|I_{i, k}\right|^{4}\left(\int \eta_{i, k}^{2}(\alpha)|f(\alpha)|^{2} d \alpha\right)^{1 / 2}\left\|\left(\frac{\eta_{i, k}}{\sqrt{\vartheta}}\right)^{\prime}\right\|_{\infty}\left(\int \vartheta(\beta)|f(\beta)|^{2} d \beta\right)^{1 / 2} .
\end{aligned}
$$

Since

$$
\left|\left(\frac{\eta_{i, k}(\alpha)}{\sqrt{\vartheta(\alpha)}}\right)^{\prime}\right|=\left|\frac{\eta_{i, k}^{\prime}(\alpha)}{\sqrt{\vartheta(\alpha)}}-\frac{\eta_{i, k}(\alpha) \vartheta^{\prime}(\alpha)}{2 \vartheta^{3 / 2}(\alpha)}\right| \leq \frac{C_{2}}{\left|I_{i, k}\right|^{2}} \chi_{2 I_{i, k}}(\alpha)
$$

for some constant $C_{2}$, we have

$$
\begin{aligned}
2\left|\iint \frac{\vartheta(\alpha) \vartheta(\beta)-\sum_{i, k}\left|I_{i, k}\right|^{4} \eta_{i, k}(\alpha) \eta_{i, k}(\beta)}{(\alpha-\beta)^{2}} f(\alpha) f(\beta) d \alpha d \beta\right| \\
\leq C_{1} C_{2} \sum_{i, k}\left|I_{i, k}\right|^{2}\left(\int \eta_{i, k}^{2}(\alpha)|f(\alpha)|^{2} d \alpha\right)^{1 / 2}\left(\int \vartheta(\beta)|f(\beta)|^{2} d \beta\right)^{1 / 2} \\
\leq C_{1} C_{2}\left(\int \sum_{i, k}\left|I_{i, k}\right|^{2} \eta_{i, k}^{2}(\alpha)|f(\alpha)|^{2} d \alpha\right)^{1 / 2}\left(\sum_{i, k}\left|I_{i, k}\right|^{2}\right)^{1 / 2} \\
\quad \cdot\left(\int \vartheta(\beta)|f(\beta)|^{2} d \beta\right)^{1 / 2} \\
\leq C_{3}|I| \int \vartheta(\alpha)|f(\alpha)|^{2} d \alpha .
\end{aligned}
$$

Let $I \subset[c, d] \subset(a, b)$ be any closed interval satisfying $|I| \leq \frac{1}{2} \delta_{1}$, and let $I_{i, k}$ be the subintervals of $I$ as above. We now apply (4.48) to $Q=I_{i, k}$ and multiply $|Q|^{4}=\left|I_{i, k}\right|^{4}$ on both sides of (4.48); then sum up with respect to $i=1,2$, $k=1,2, \ldots$ While going through similar arguments as in the proof of Proposition 3.2 , we apply the calculations of (4.49) through (4.53). We get

$$
\begin{aligned}
& \frac{1}{36 l^{2}} \int_{0}^{l} h \int \zeta(t) \int \vartheta^{2}(\alpha)\left|z_{t h h}(\alpha, t)\right|^{2} d \alpha d t d h \\
& +\frac{v}{36} \frac{1}{l^{2}} \int_{0}^{l} h \int \zeta(t) \int \vartheta^{2}(\alpha)\left|z_{\alpha h h}(\alpha, t)\right|^{2} d \alpha d t d h \\
& \leq \frac{T_{1}^{2}}{\pi l^{2}} \operatorname{Im} \int_{0}^{l} h \iint \frac{1}{(\alpha-\beta)^{2}}\left(\vartheta(\alpha) \frac{z_{h h}(\alpha, 0)}{z_{h}(\alpha, 0)}-\vartheta(\beta) \frac{z_{h h}(\beta, 0)}{z_{h}(\beta, 0)}\right)^{2} d \alpha d \beta d h \\
& \quad+C_{4} \frac{T_{1}^{2}|I|}{\pi l^{2}} \int_{0}^{l} h \int \vartheta(\alpha)\left|z_{h h}(\alpha, 0)\right|^{2} d \alpha d h+K,
\end{aligned}
$$

where $C_{4}$ is a constant depending on $m$ and $M$, and $K$ depends on

$$
\int_{0}^{T_{1}} \int_{c+\frac{1}{2} \delta_{1}}^{d-\frac{1}{2} \delta_{1}}\left|z_{\alpha \alpha}(\alpha, t)\right|^{2} d \alpha d t
$$


$m, M, T_{1}$, and $|I|$. As in the proof of the $H^{1 / 2}$ compatibility of the initial data, we rewrite (4.54) as

$$
\begin{aligned}
& \frac{1}{36 l^{2}} \int_{0}^{l} h \int \zeta(t) \int \vartheta^{2}(\alpha)\left|z_{t h h}(\alpha, t)\right|^{2} d \alpha d t d h \\
& +\frac{v}{36} \frac{1}{l^{2}} \int_{0}^{l} h \int \zeta(t) \int \vartheta^{2}(\alpha)\left|z_{\alpha h h}(\alpha, t)\right|^{2} d \alpha d t d h \\
& +\frac{T_{1}^{2}}{\pi l^{2}} \int_{0}^{l} h \iint \frac{1}{(\alpha-\beta)^{2}}\left|\vartheta(\alpha)\left(\left[\ln z_{\alpha}\right]_{h}\right)_{h}(\alpha, 0)-\vartheta(\beta)\left(\left[\ln z_{\beta}\right]_{h}\right)_{h}(\beta, 0)\right|^{2} d \alpha d \beta d h \\
& \quad \leq \frac{T_{1}^{2}}{\pi l^{2}} \operatorname{Im} \int_{0}^{l} h \iint \frac{1}{(\alpha-\beta)^{2}}\left(\vartheta(\alpha) \frac{z_{h h}(\alpha, 0)}{z_{h}(\alpha, 0)}-\vartheta(\beta) \frac{z_{h h}(\beta, 0)}{z_{h}(\beta, 0)}\right)^{2} d \alpha d \beta d h \\
& \quad-\frac{T_{1}^{2}}{\pi l^{2}} \operatorname{Im} \int_{0}^{l} h \iint \frac{1}{(\alpha-\beta)^{2}}\left(\vartheta(\alpha)\left(\left[\ln z_{\alpha}\right]_{h}\right)_{h}(\alpha, 0)\right. \\
& \left.\quad-\vartheta(\beta)\left(\left[\ln z_{\beta}\right]_{h}\right)_{h}(\beta, 0)\right)^{2} d \alpha d \beta d h \\
& \quad+\frac{T_{1}^{2}}{\pi l^{2}} \int_{0}^{l} h \iint \frac{1}{(\alpha-\beta)^{2}}\left(\vartheta(\alpha)\left([w]_{h}\right)_{h}(\alpha)-\vartheta(\beta)\left([w]_{h}\right)_{h}(\beta)\right)^{2} d \alpha d \beta d h \\
& \quad+C_{4} \frac{T_{1}^{2}|I|}{\pi l^{2}} \int_{0}^{l} h \int \vartheta(\alpha)\left|z_{h h}(\alpha, 0)\right|^{2} d \alpha d h+K,
\end{aligned}
$$

where $w(\alpha)=\operatorname{Im}\left((1+i) \ln z_{\alpha}(\alpha, 0)\right)$. From the assumption that $w \in H_{\mathrm{loc}}^{3 / 2}(a, b)$, we know

$$
\begin{aligned}
& \frac{T_{1}^{2}}{\pi l^{2}} \int_{0}^{l} h \iint \frac{1}{(\alpha-\beta)^{2}}\left(\vartheta(\alpha)\left([w]_{h}\right)_{h}(\alpha)-\vartheta(\beta)\left([w]_{h}\right)_{h}(\beta)\right)^{2} d \alpha d \beta d h \\
& \leq K_{1}<\infty
\end{aligned}
$$

for some constant $K_{1}$ independent of $l, 0<|l|<\frac{1}{4} \delta_{1}$.

Our main effort from now on is to show that the quantity

$$
\begin{gathered}
\frac{T_{1}^{2}}{\pi l^{2}} \operatorname{Im} \int_{0}^{l} h \iint \frac{1}{(\alpha-\beta)^{2}}\left(\vartheta(\alpha) \frac{z_{h h}(\alpha, 0)}{z_{h}(\alpha, 0)}-\vartheta(\beta) \frac{z_{h h}(\beta, 0)}{z_{h}(\beta, 0)}\right)^{2} d \alpha d \beta d h \\
-\frac{T_{1}^{2}}{\pi l^{2}} \operatorname{Im} \int_{0}^{l} h \iint \frac{1}{(\alpha-\beta)^{2}}\left(\vartheta(\alpha)\left(\left[\ln z_{\alpha}\right]_{h}\right)_{h}(\alpha, 0)\right. \\
\left.-\vartheta(\beta)\left(\left[\ln z_{\beta}\right]_{h}\right)_{h}(\beta, 0)\right)^{2} d \alpha d \beta d h
\end{gathered}
$$

can be dominated by the left-hand side of (4.55) as long as $\left\|\ln z_{\alpha}(\cdot, 0)\right\|_{\mathrm{BMO}(a, b), \delta_{0}}$ is small. We would also need $|I|$ small, but $I \subset[c, d]$ is otherwise arbitrary. We will give an estimate for $\int \vartheta(\alpha)\left|z_{h h}(\alpha, 0)\right|^{2} d \alpha$, since so far we only have $z_{\alpha}(\cdot, 0) \in H_{\mathrm{loc}}^{1 / 2}(a, b)$ from Proposition 3.3. 
Consider

$$
\begin{aligned}
& \iint \frac{1}{(\alpha-\beta)^{2}}\left(\vartheta(\alpha) \frac{z_{h h}(\alpha, 0)}{z_{h}(\alpha, 0)}-\vartheta(\beta) \frac{z_{h h}(\beta, 0)}{z_{h}(\beta, 0)}\right)^{2} d \alpha d \beta \\
& \quad-\iint \frac{1}{(\alpha-\beta)^{2}}\left(\vartheta(\alpha)\left(\left[\ln z_{\alpha}\right]_{h}\right)_{h}(\alpha, 0)-\vartheta(\beta)\left(\left[\ln z_{\beta}\right]_{h}\right)_{h}(\beta, 0)\right)^{2} d \alpha d \beta .
\end{aligned}
$$

We have

$$
\begin{aligned}
& \mid \iint \frac{1}{(\alpha-\beta)^{2}}\left(\vartheta(\alpha) \frac{z_{h h}(\alpha, 0)}{z_{h}(\alpha, 0)}-\vartheta(\beta) \frac{z_{h h}(\beta, 0)}{z_{h}(\beta, 0)}\right)^{2} d \alpha d \beta \\
& -\iint \frac{1}{(\alpha-\beta)^{2}}\left(\vartheta(\alpha)\left(\left[\ln z_{\alpha}\right]_{h}\right)_{h}(\alpha, 0)-\vartheta(\beta)\left(\left[\ln z_{\beta}\right]_{h}\right)_{h}(\beta, 0)\right)^{2} d \alpha d \beta \mid \\
& \quad \leq 3 \iint \frac{1}{(\alpha-\beta)^{2}} \mid \vartheta(\alpha)\left(\frac{z_{h h}}{z_{h}}-\left(\left[\ln z_{\alpha}\right]_{h}\right)_{h}\right)(\alpha, 0) \\
& \quad-\left.\vartheta(\beta)\left(\frac{z_{h h}}{z_{h}}-\left(\left[\ln z_{\beta}\right]_{h}\right)_{h}\right)(\beta, 0)\right|^{2} d \alpha d \beta \\
& \quad+\frac{1}{2} \iint \frac{1}{(\alpha-\beta)^{2}}\left|\vartheta(\alpha)\left(\left[\ln z_{\alpha}\right]_{h}\right)_{h}(\alpha, 0)-\vartheta(\beta)\left(\left[\ln z_{\beta}\right]_{h}\right)_{h}(\beta, 0)\right|^{2} d \alpha d \beta .
\end{aligned}
$$

We only need to estimate

$$
\begin{aligned}
\iint \frac{1}{(\alpha-\beta)^{2}} \mid \vartheta(\alpha)\left(\frac{z_{h h}(\alpha, 0)}{z_{h}(\alpha, 0)}-\left(\left[\ln z_{\alpha}\right]_{h}\right)_{h}(\alpha, 0)\right) \\
-\left.\vartheta(\beta)\left(\frac{z_{h h}(\beta, 0)}{z_{h}(\beta, 0)}-\left(\left[\ln z_{\beta}\right]_{h}\right)_{h}(\beta, 0)\right)\right|^{2} d \alpha d \beta
\end{aligned}
$$

Now

$$
\begin{aligned}
& \iint \frac{1}{(\alpha-\beta)^{2}} \\
& .\left|\vartheta(\alpha)\left(\frac{z_{h h}}{z_{h}}-\left(\left[\ln z_{\alpha}\right]_{h}\right)_{h}\right)(\alpha, 0)-\vartheta(\beta)\left(\frac{z_{h h}}{z_{h}}-\left(\left[\ln z_{\beta}\right]_{h}\right)_{h}\right)(\beta, 0)\right|^{2} d \alpha d \beta \\
& =2 \iint \frac{\vartheta(\alpha) \vartheta^{\prime}(\beta)}{\alpha-\beta} \overline{\left(\frac{z_{h h}(\alpha, 0)}{z_{h}(\alpha, 0)}-\left(\left[\ln z_{\alpha}\right]_{h}\right)_{h}(\alpha, 0)\right)} \\
& \quad \cdot\left(\frac{z_{h h}(\beta, 0)}{z_{h}(\beta, 0)}-\left(\left[\ln z_{\beta}\right]_{h}\right)_{h}(\beta, 0)\right) d \alpha d \beta \\
& +2 \iint \frac{\vartheta(\alpha) \vartheta(\beta)}{\alpha-\beta} \overline{\left(\frac{z_{h h}(\alpha, 0)}{z_{h}(\alpha, 0)}-\left(\left[\ln z_{\alpha}\right]_{h}\right)_{h}(\alpha, 0)\right)} \\
& \quad \cdot \partial_{\beta}\left(\frac{z_{h h}(\beta, 0)}{z_{h}(\beta, 0)}-\left(\left[\ln z_{\beta}\right]_{h}\right)_{h}(\beta, 0)\right) d \alpha d \beta,
\end{aligned}
$$


where

$$
\begin{aligned}
2 \mid \iint & \frac{\vartheta(\alpha) \vartheta^{\prime}(\beta)}{\alpha-\beta} \overline{\left(\frac{z_{h h}(\alpha, 0)}{z_{h}(\alpha, 0)}-\left(\left[\ln z_{\alpha}\right]_{h}\right)_{h}(\alpha, 0)\right)} \\
& \cdot\left(\frac{z_{h h}(\beta, 0)}{z_{h}(\beta, 0)}-\left(\left[\ln z_{\beta}\right]_{h}\right)_{h}(\beta, 0)\right) d \alpha d \beta \mid \\
\leq & C_{5}|I|\left(\int \vartheta(\alpha)\left|z_{h h}(\alpha, 0)\right|^{2} d \alpha+\int \vartheta(\alpha)\left|\left(\left[\ln z_{\alpha}\right]_{h}\right)_{h}(\alpha, 0)\right|^{2} d \alpha\right)
\end{aligned}
$$

for some constant $C_{5}$ depending on $m$ and $M$.

We estimate the second term on the right-hand side of (4.57). We write

$$
\begin{aligned}
& \partial_{\beta}\left(\frac{z_{h h}(\beta, 0)}{z_{h}(\beta, 0)}-\left(\left[\ln z_{\beta}\right]_{h}\right)_{h}(\beta, 0)\right) \\
& \quad=\partial_{\beta}\left(\frac{z_{h h}(\beta, 0)}{z_{h}(\beta, 0)}\right)-\left(\frac{z_{\beta h}(\beta, 0)}{z_{\beta}(\beta, 0)}\right)_{h}+\left(\frac{z_{\beta h}(\beta, 0)}{z_{\beta}(\beta, 0)}\right)_{h}-\left(\ln z_{\beta}\right)_{h h}(\beta, 0) \\
& \quad=\mathrm{VII}_{1}+\mathrm{VII}_{2}
\end{aligned}
$$

where

(4.60)

$$
\begin{aligned}
\mathrm{VII}_{1}= & \partial_{\beta}\left(\frac{z_{h h}(\beta, 0)}{z_{h}(\beta, 0)}\right)-\left(\frac{z_{\beta h}(\beta, 0)}{z_{\beta}(\beta, 0)}\right)_{h} \\
= & z_{\beta h h}(\beta, 0)\left(\frac{1}{z_{h}(\beta, 0)}-\frac{1}{z_{\beta}(\beta, 0)}\right)-\frac{z_{\beta h}(\beta, 0) z_{h h}(\beta, 0)}{z_{h}^{2}(\beta, 0)} \\
& +\frac{z_{\beta h}(\beta, 0) z_{\beta h}(\beta+h, 0)}{z_{\beta}(\beta, 0) z_{\beta}(\beta+h, 0)} \\
= & z_{\beta h h}(\beta, 0)\left(\frac{2}{z_{h}(\beta, 0)}-\frac{1}{z_{\beta}(\beta, 0)}-\frac{1}{z_{2 h}(\beta, 0)}\right) \\
& -z_{\beta h h}(\beta, 0)\left(\frac{1}{z_{h}(\beta, 0)}-\frac{1}{z_{2 h}(\beta, 0)}\right) \\
& -\frac{\left(z_{\beta h}(\beta, 0)-z_{h h}(\beta, 0)\right) z_{h h}(\beta, 0)}{z_{h}^{2}(\beta, 0)}-\frac{z_{h h}^{2}(\beta, 0)}{z_{h}^{2}(\beta, 0)} \\
& +\frac{\left(z_{\beta h}(\beta, 0)-z_{h h}(\beta, 0)\right)\left(z_{\beta h}(\beta+h, 0)-z_{h h}(\beta+h, 0)\right)}{z_{\beta}(\beta, 0) z_{\beta}(\beta+h, 0)} \\
& +\frac{z_{h h}(\beta, 0) z_{h h}(\beta+h, 0)}{z_{\beta}(\beta, 0) z_{\beta}(\beta+h, 0)} \\
& +z_{h h}(\beta+h, 0) \frac{z_{\beta h}(\beta, 0)-z_{h h}(\beta, 0)}{z_{\beta}(\beta, 0) z_{\beta}(\beta+h, 0)} \\
& +z_{h h}(\beta, 0) \frac{z_{\beta h}(\beta+h, 0)-z_{h h}(\beta+h, 0)}{z_{\beta}(\beta, 0) z_{\beta}(\beta+h, 0)}
\end{aligned}
$$


and

$$
\begin{aligned}
\mathrm{VII}_{2}= & \frac{1}{h}\left(\frac{z_{\beta}(\beta+h, 0)}{z_{\beta}(\beta, 0)}-1-\ln z_{\beta}(\beta+h, 0)+\ln z_{\beta}(\beta, 0)\right)_{h} \\
= & \left(\left(\int_{0}^{1} e^{r\left(\ln z_{\beta}(\beta+h, 0)-\ln z_{\beta}(\beta, 0)\right)} d r-1\right)\left(\ln z_{\beta}\right)_{h}(\beta, 0)\right)_{h} \\
= & \left(\int_{0}^{1} e^{r h\left(\ln z_{\beta}\right)_{h}(\beta, 0)} d r-1\right)\left(\ln z_{\beta}\right)_{h h}(\beta, 0) \\
& +\left(\int_{0}^{1} e^{r h\left(\ln z_{\beta}\right)_{h}(\beta, 0)} d r-1\right)_{h}\left(\ln z_{\beta}\right)_{h}(\beta+h, 0) \\
= & \left(\ln z_{\beta}\right)_{h h}(\beta, 0) \int_{0}^{1}\left(e^{r h\left(\ln z_{\beta}\right)_{h}(\beta, 0)}-e^{r h\left(\left[\ln z_{\beta}\right]_{h}\right)_{h}(\beta, 0)}\right) d r \\
& +\left(\ln z_{\beta}\right)_{h h}(\beta, 0)\left(\int_{0}^{1} e^{r h\left(\left[\ln z_{\beta}\right]_{h}\right)_{h}(\beta, 0)} d r-1\right) \\
& +\frac{1}{h}\left(\left(\ln z_{\beta}\right)_{h}-\left(\left[\ln z_{\beta}\right]_{h}\right)_{h}\right)(\beta+h, 0) \\
& \cdot \int_{0}^{1}\left(e^{r h\left(\ln z_{\beta}\right)_{h}(\beta+h, 0)}-e^{r h\left(\ln z_{\beta}\right)_{h}(\beta, 0)}\right) d r \\
& +\frac{1}{h}\left(\left[\ln z_{\beta}\right]_{h}\right)_{h}(\beta+h, 0) \int_{0}^{1}\left(e^{r h\left(\ln z_{\beta}\right)_{h}(\beta+h, 0)}-e^{r h\left(\ln z_{\beta}\right)_{h}(\beta, 0)}\right) d r .
\end{aligned}
$$

\section{On the other hand,}

$$
\begin{aligned}
\frac{z_{h h}(\alpha, 0)}{z_{h}(\alpha, 0)}-\left(\left[\ln z_{\alpha}\right]_{h}\right)_{h}(\alpha, 0) \\
=\frac{z_{h h}(\alpha, 0)}{z_{h}(\alpha, 0)}-\left[\frac{z_{\alpha h}}{z_{\alpha}}\right]_{h}(\alpha, 0)+\left[\frac{z_{\alpha h}}{z_{\alpha}}-\left(\ln z_{\alpha}\right)_{h}\right]_{h}(\alpha, 0) \\
=\frac{1}{h} \int_{0}^{h} z_{\alpha h}(\alpha+r, 0)\left(\frac{1}{z_{h}(\alpha, 0)}-\frac{1}{z_{\alpha}(\alpha+r, 0)}\right) d r \\
+\left[\left(\int_{0}^{1} e^{r\left(\ln z_{\alpha}(\alpha+h, 0)-\ln z_{\alpha}(\alpha, 0)\right)} d r-1\right)\left(\ln z_{\alpha}\right)_{h}(\alpha, 0)\right]_{h} \\
=\frac{1}{h} \int_{0}^{h}\left(z_{\alpha h}(\alpha+r, 0)-z_{h h}(\alpha+r, 0)\right)\left(\frac{1}{z_{h}(\alpha, 0)}-\frac{1}{z_{\alpha}(\alpha+r, 0)}\right) d r \\
+\frac{1}{h} \int_{0}^{h} z_{h h}(\alpha+r, 0)\left(\frac{2}{z_{h}(\alpha+r, 0)}-\frac{1}{z_{\alpha}(\alpha+r, 0)}-\frac{1}{z_{2 h}(\alpha+r, 0)}\right) d r \\
+\frac{1}{h} \int_{0}^{h} z_{h h}(\alpha+r, 0)\left(\frac{1}{z_{h}(\alpha, 0)}-\frac{2}{z_{h}(\alpha+r, 0)}+\frac{1}{z_{2 h}(\alpha+r, 0)}\right) d r \\
+\left[\left(\left(\ln z_{\alpha}\right)_{h}(\alpha, 0)-\left[\left(\ln z_{\alpha}\right)_{h}\right]_{h}(\alpha, 0)\right)\left(\int_{0}^{1} e^{r h\left(\ln z_{\alpha}\right)_{h}(\alpha, 0)} d r-1\right)\right]_{h} \\
+\left[\left[\left(\ln z_{\alpha}\right)_{h}\right]_{h}(\alpha, 0) \int_{0}^{1}\left(e^{r h\left(\ln z_{\alpha}\right)_{h}(\alpha, 0)}-e^{r h\left[\left(\ln z_{\alpha}\right)_{h}\right]_{h}(\alpha, 0)}\right) d r\right]_{h} \\
+\left[\left[\left(\ln z_{\alpha}\right)_{h}\right]_{h}(\alpha, 0)\left(\int_{0}^{1} e^{r h\left[\left(\ln z_{\alpha}\right)_{h}\right]_{h}(\alpha, 0)} d r-1\right)\right]_{h}
\end{aligned}
$$


Let the interval $I=[p, q]$. Let $\varrho \in C_{0}^{\infty}(\mathbb{R})$ be such that

$$
\begin{cases}\varrho(\alpha)=1 & \text { for } \alpha \in\left[p-\frac{1}{4} \delta_{1}, q+\frac{1}{4} \delta_{1}\right] \\ \varrho(\alpha)=0 & \text { for } \alpha \notin\left[p-\frac{1}{2} \delta_{1}, q+\frac{1}{2} \delta_{1}\right]\end{cases}
$$

and $0 \leq \varrho(\alpha) \leq 1$ for all $\alpha \in \mathbb{R}$. We note that for any function $f, \alpha \in \mathbb{R}$, $0<|h| \leq \frac{1}{16} \delta_{1}$,

(4.64) $\vartheta(\alpha) f_{h h}(\alpha)=(\vartheta f)_{h h}(\alpha)-2 \vartheta_{h}(\alpha)(\varrho f)_{h}(\alpha+h)-\vartheta_{h h}(\alpha)(\varrho f)(\alpha+2 h)$.

From the definition of $\vartheta$, it is clear that $\left\|\vartheta^{\prime}\right\|_{\infty} \leq C|I|$ and $\left\|\vartheta^{\prime \prime}\right\|_{\infty} \leq C$ for some constant $C<\infty$. Therefore for $\alpha, \gamma \in \mathbb{R}$,

$$
|\vartheta(\alpha+\gamma)-\vartheta(\alpha)| \leq\left\|\vartheta^{\prime}\right\|_{\infty}|\gamma| \text {. }
$$

We apply an argument such as that in (3.70) to (4.57), using the new forms of the involved terms in (4.59) through (4.62), and Lemma 4.3, (4.16), (4.64), and (4.65). We get, for $0<|l| \leq \frac{1}{16} \delta_{1}$,

$$
\begin{aligned}
& \frac{2}{l^{2}} \int_{0}^{l} h \iint \frac{\vartheta(\alpha) \vartheta(\beta)}{\alpha-\beta} \overline{\left(\frac{z_{h h}}{z_{h}}-\left(\left[\ln z_{\alpha}\right]_{h}\right)_{h}\right)(\alpha, 0)} \\
& \quad \cdot \partial_{\beta}\left(\frac{z_{h h}}{z_{h}}-\left(\left[\ln z_{\beta}\right]_{h}\right)_{h}\right)(\beta, 0) d \alpha d \beta d h \\
& \leq C_{6}\left\|\ln z_{\alpha}(\cdot, 0)\right\|_{*, \delta_{0}} \frac{1}{l^{2}} \int_{0}^{l} h \int h\left|\left(\vartheta z_{\alpha}\right)_{h h}(\alpha, 0)\right|^{2} d \alpha d h \\
& +C_{6}\left\|\ln z_{\alpha}(\cdot, 0)\right\|_{*, \delta_{0}} \frac{1}{l^{2}} \int_{0}^{l} h \int h\left|\left(\vartheta \ln z_{\alpha}\right)_{h h}(\alpha, 0)\right|^{2} d \alpha d h \\
& +C_{6} \frac{1}{l^{2}} \int_{0}^{l} h \int \vartheta^{2}(\alpha)\left(\left|z_{h h}(\alpha, 0)\right|^{3}+\left|\left(\left[\ln z_{\alpha}\right]_{h}\right)_{h}(\alpha, 0)\right|^{3}\right) d \alpha d h \\
& +C_{7}\left(1+\left\|\ln z_{\alpha}(\cdot, 0)\right\|_{*, \delta_{0}}\right)\left\|\vartheta^{\prime}\right\|_{\infty}^{2} \\
& \quad \cdot \int\left(\left.\left.|| D\right|^{1 / 2}\left(\varrho z_{\alpha}\right)(\alpha, 0)\right|^{2}+\left.\left.|| D\right|^{1 / 2}\left(\varrho \ln z_{\alpha}\right)(\alpha, 0)\right|^{2}\right) d \alpha \\
& +C_{8}\left\|\vartheta^{\prime \prime}\right\|_{\infty}^{2}|l| \int_{p-\frac{1}{2} \delta_{1}}^{q+\frac{1}{2} \delta_{1}}\left(\left|z_{\alpha}(\alpha, 0)\right|^{2}+\left|\ln z_{\alpha}(\alpha, 0)\right|^{2}\right) d \alpha,
\end{aligned}
$$

where $C_{6}, C_{7}$, and $C_{8}$ are constants depending on $m$ and $M$. We further need to estimate the terms

$$
\frac{1}{l^{2}} \int_{0}^{l} h \int \vartheta^{2}(\alpha)\left(\left|z_{h h}(\alpha, 0)\right|^{3}+\left|\left(\left[\ln z_{\alpha}\right]_{h}\right)_{h}(\alpha, 0)\right|^{3}\right) d \alpha d h
$$

in (4.66) and

$$
\frac{|I|}{l^{2}} \int_{0}^{l} h \int \vartheta(\alpha)\left|z_{h h}(\alpha, 0)\right|^{2} d \alpha+\int \vartheta(\alpha)\left|\left(\left[\ln z_{\alpha}\right]_{h}\right)_{h}(\alpha, 0)\right|^{2} d \alpha d h
$$


in (4.58) and (4.55).

Before we proceed, we prove the following lemma:

Lemma 4.6 Let $f: \mathbb{R} \rightarrow \mathbb{C}$ be periodic of period $A$ and $f \in H_{\mathrm{loc}}^{1 / 2}(\mathbb{R})$. Let $\sigma \in C_{0}^{\infty}(\mathbb{R})$ such that $0 \leq \sigma(\alpha) \leq 1, \sigma(\alpha)=1$ for $\alpha \in[0, A], \sigma(\alpha)=0$ for $\alpha \notin[-A, 2 A]$, and $\left|\sigma^{\prime}(\alpha)\right| \leq 2 / A$. Then there exist constants $c_{1}$ and $c_{2}$ such that

$$
\begin{aligned}
\left.\iint_{0}^{\infty}|\sigma(\alpha)|^{2} \mid([f]]_{h}\right)\left._{h}(\alpha)\right|^{2} d h d \alpha & \\
& \leq\left.\left. c_{1} \int|| D\right|^{1 / 2}(\sigma f)\right|^{2} d \alpha+\frac{c_{2}}{A} \int_{0}^{A}|f|^{2} d \alpha<\infty
\end{aligned}
$$

and

$$
\begin{array}{rl}
\iint_{0}^{\infty}|\sigma(\alpha)|^{2}\left|f_{h}(\alpha)\right|^{2} & d h d \alpha \\
& \leq\left.\left. c_{1} \int|| D\right|^{1 / 2}(\sigma f)\right|^{2} d \alpha+\frac{c_{2}}{A} \int_{0}^{A}|f|^{2} d \alpha<\infty .
\end{array}
$$

ProOF OF (4.67): We know

$$
\begin{aligned}
& \sigma(\alpha)\left([f]_{h}\right)_{h}(\alpha) \\
& =\left[(\sigma f)_{h}\right]_{h}-\frac{1}{h^{2}} \int_{0}^{h}(\sigma(\alpha+h+r)-\sigma(\alpha)) f(\alpha+h+r) d r \\
& \quad+\frac{1}{h^{2}} \int_{0}^{h}(\sigma(\alpha+r)-\sigma(\alpha)) f(\alpha+r) d r .
\end{aligned}
$$

Now from the Schwarz inequality, in particular, $\left|\int_{0}^{h} f(x) d x\right|^{2} \leq h \int_{0}^{h}|f(x)|^{2} d x$,

$$
\begin{aligned}
& \iint_{0}^{\infty}\left|\frac{1}{h^{2}} \int_{0}^{h}(\sigma(\alpha+r)-\sigma(\alpha)) f(\alpha+r) d r\right|^{2} d h d \alpha \\
& \leq \iint_{0}^{\infty} \frac{1}{h^{3}} \int_{0}^{h}|\sigma(\alpha+r)-\sigma(\alpha)|^{2}|f(\alpha+r)|^{2} d r d h d \alpha \\
& \leq \frac{4}{A^{2}} \iint_{0}^{A} \frac{1}{h^{3}} \int_{0}^{h} r^{2}\left|\chi_{[-A, 3 A]}(\alpha+r) f(\alpha+r)\right|^{2} d r d h d \alpha \\
& \quad+2 \iint_{A}^{\infty} \frac{1}{h^{3}} \int_{0}^{h}\left(|\sigma(\alpha+r)|^{2}+|\sigma(\alpha)|^{2}\right)|f(\alpha+r)|^{2} d r d h d \alpha \\
& \leq \frac{16}{3 A} \int_{0}^{A}|f(\alpha)|^{2} d \alpha+\frac{12}{A} \int_{0}^{A}|f(\alpha)|^{2} d \alpha .
\end{aligned}
$$


Similarly, we have

$$
\begin{array}{rl}
\iint_{0}^{\infty}\left|\frac{1}{h^{2}} \int_{0}^{h}(\sigma(\alpha+h+r)-\sigma(\alpha)) f(\alpha+h+r) d r\right|^{2} & d h d \alpha \\
& \leq \frac{92}{A} \int_{0}^{A}|f(\alpha)|^{2} d \alpha .
\end{array}
$$

On the other hand, using Fourier analysis and Plancherel's theorem, we obtain

$$
\begin{aligned}
& \iint_{0}^{\infty}\left|\left[(\sigma f)_{h}\right]_{h}\right|^{2} d h d \alpha \\
& \quad=c^{\prime} \int\left(\int_{0}^{\infty} \frac{\left|e^{i h \xi}-1\right|^{4}}{h^{4}|\xi|^{2}} d h\right)|\widehat{(\sigma f)}(\xi)|^{2} d \xi=\left.\left.c^{\prime \prime} \int|| D\right|^{1 / 2}(\sigma f)(\alpha)\right|^{2} d \alpha,
\end{aligned}
$$

where $c^{\prime}$ and $c^{\prime \prime}$ are constants. This proves (4.67). Inequality (4.68) can be proved similarly. We omit the details.

Let $Z: \mathbb{R} \rightarrow \mathbb{C}$ be the reflective periodic extension of $z(\cdot, 0)$ from $\left[c-\frac{1}{2} \delta_{1}\right.$, $\left.d+\frac{1}{2} \delta_{1}\right]$ to $\mathbb{R}$. Notice that for $\alpha \in \mathbb{R}$ and $0<|h| \leq \frac{1}{16} \delta_{1}$,

$$
\vartheta(\alpha) z_{h h}(\alpha, 0)=\vartheta(\alpha) Z_{h h}(\alpha)
$$

and

$$
\vartheta(\alpha)\left[\left(\ln z_{\alpha}\right)_{h}\right]_{h}(\alpha, 0)=\vartheta(\alpha)\left[\left(\ln Z_{\alpha}\right)_{h}\right]_{h}(\alpha) .
$$

Moreover, from Proposition 3.3, $Z_{\alpha}, \ln Z_{\alpha} \in H_{\text {loc }}^{1 / 2}(\mathbb{R})$.

We now estimate $\int \vartheta^{2}(\alpha)\left|\left[f_{h}\right]_{h}(\alpha)\right|^{3} d \alpha$ for $f=Z_{\alpha}$ or $f=\ln Z_{\alpha}$. We know $f \in H_{\mathrm{loc}}^{1 / 2}(\mathbb{R})$ and $f$ is periodic with period

$$
A=2 d-2 c+2 \delta_{1} \text {. }
$$

Without loss of generality, we assume $0<h \leq \frac{1}{16} \delta_{1}$. We have

$$
\begin{aligned}
&\left.\int \vartheta^{2}(\alpha) \mid\left[f_{h}\right]\right]\left._{h}(\alpha)\right|^{3} d \alpha \\
& \leq 3 \iint_{h}^{\infty} \vartheta^{2}(\alpha)\left|\left[f_{\kappa}\right]_{\kappa}(\alpha)\right|^{2}\left|\frac{d}{d \kappa}\left[f_{\kappa}\right]_{\kappa}(\alpha)\right| d \kappa d \alpha \\
& \leq 3\left(\iint_{h}^{\infty} \vartheta^{2}(\alpha)\left|\left[f_{\kappa}\right]_{\kappa}(\alpha)\right|^{4} d \kappa d \alpha\right)^{1 / 2}\left(\iint_{h}^{\infty} \vartheta^{2}(\alpha)\left|\frac{d}{d \kappa}\left[f_{\kappa}\right]_{\kappa}(\alpha)\right|^{2} d \kappa d \alpha\right)^{1 / 2} \\
& \leq 6\left(\iint_{h}^{\infty}\left|\left[f_{\kappa}\right]_{\kappa}(\alpha)\right|^{2} d \kappa d \alpha\right)^{1 / 2} \\
& \cdot \iint_{h}^{\infty} \vartheta^{2}(\alpha)\left(\left|f_{\kappa \kappa}(\alpha)\right|^{2}+\left|\frac{d}{d \kappa}\left[f_{\kappa}\right]_{\kappa}(\alpha)\right|^{2}\right) d \kappa d \alpha \\
&+C|I|\left(\iint_{h}^{\infty}\left|\left[f_{\kappa}\right]_{\kappa}(\alpha)\right|^{2} d \kappa d \alpha\right)\left(\iint_{h}^{\infty} \vartheta^{2}(\alpha)\left|\frac{d}{d \kappa}\left[f_{\kappa}\right]_{\kappa}(\alpha)\right|^{2} d \kappa d \alpha\right)^{1 / 2} .
\end{aligned}
$$

Here we used Lemma 4.2. $C$ is a universal constant. 
We know

$$
\frac{d}{d \kappa}\left[f_{\kappa}\right]_{\kappa}(\alpha)=\frac{2}{\kappa}\left(f_{\kappa}(\alpha+\kappa)-\left[f_{\kappa}\right]_{\kappa}(\alpha)\right) .
$$

We compute further the term $\iint_{h}^{\infty} \vartheta^{2}(\alpha)\left(\left|f_{\kappa \kappa}(\alpha)\right|^{2}+\left|\frac{d}{d \kappa}\left[f_{\kappa}\right]_{\kappa}(\alpha)\right|^{2}\right) d \kappa d \alpha$. Notice that

$$
\vartheta(\alpha) f_{\kappa \kappa}(\alpha)=(\vartheta f)_{\kappa \kappa}(\alpha)-2 \vartheta_{\kappa}(\alpha) f_{\kappa}(\alpha+\kappa)-\vartheta_{\kappa \kappa}(\alpha) f(\alpha+2 \kappa) .
$$

Let $\sigma(\alpha)$ be as defined in Lemma 4.6. We have

$$
\begin{aligned}
& \iint_{h}^{\infty} \vartheta_{\kappa}^{2}(\alpha)\left|f_{\kappa}(\alpha+k)\right|^{2} d \kappa d \alpha \\
& \leq \leq \iint_{0}^{|I|} \vartheta_{\kappa}^{2}(\alpha)\left|f_{\kappa}(\alpha+k)\right|^{2} d \kappa d \alpha \\
& \quad+2 \iint_{|I|}^{\infty} \frac{1}{\kappa^{2}}\left(|\vartheta(\alpha+\kappa)|^{2}+|\vartheta(\alpha)|^{2}\right)\left|f_{\kappa}(\alpha+\kappa)\right|^{2} d \kappa d \alpha \\
& \leq \iint_{0}^{|I|}\left\|\vartheta^{\prime}\right\|_{\infty}^{2}\left|\chi_{3 I}(\alpha+\kappa) f_{\kappa}(\alpha+\kappa)\right|^{2} d \kappa d \alpha \\
& \quad+4 \iint_{|I|}^{\infty} \frac{\|\vartheta\|_{\infty}^{2}}{\kappa^{2}}\left|\sigma(\alpha) f_{\kappa}(\alpha)\right|^{2} d \kappa d \alpha \\
& \leq C|I|^{2} \iint_{0}^{\infty}\left|\sigma(\alpha) f_{\kappa}(\alpha)\right|^{2} d \kappa d \alpha .
\end{aligned}
$$

Similarly,

$$
\iint_{h}^{\infty}\left|\vartheta_{\kappa \kappa}(\alpha) f(\alpha+2 \kappa)\right|^{2} d \kappa d \alpha \leq C|I| \int|\sigma(\alpha) f(\alpha)|^{2} d \alpha .
$$

Here $C$ is some universal constant. Therefore

$$
\begin{aligned}
\iint_{h}^{\infty} \vartheta^{2}(\alpha)\left|f_{\kappa \kappa}(\alpha)\right|^{2} d \kappa d \alpha \leq & 3 \iint_{h}^{\infty}\left|(\vartheta f)_{\kappa \kappa}(\alpha)\right|^{2} d \kappa d \alpha \\
& +3 C|I|^{2} \iint_{0}^{\infty}\left|\sigma(\alpha) f_{\kappa}(\alpha)\right|^{2} d \kappa d \alpha \\
& +3 C|I| \int|\sigma(\alpha) f(\alpha)|^{2} d \alpha .
\end{aligned}
$$

Using (4.72) and a similar calculation, we get

$$
\begin{aligned}
\iint_{h}^{\infty} \vartheta^{2}(\alpha)\left|\frac{d}{d \kappa}\left[f_{\kappa}\right]_{\kappa}(\alpha)\right|^{2} d \kappa d \alpha \leq & 3 \iint_{h}^{\infty}\left|\frac{d}{d \kappa}\left[(\vartheta f)_{\kappa}\right]_{\kappa}(\alpha)\right|^{2} d \kappa d \alpha \\
& +3 C^{\prime}|I|^{2} \iint_{0}^{\infty}\left|\sigma(\alpha) f_{\kappa}(\alpha)\right|^{2} d \kappa d \alpha \\
& +3 C^{\prime}|I| \int|\sigma(\alpha) f(\alpha)|^{2} d \alpha
\end{aligned}
$$


for some universal constant $C^{\prime}$. We further apply Lemma 4.6 to

$$
\iint_{0}^{\infty}\left|\sigma(\alpha) f_{\kappa}(\alpha)\right|^{2} d \kappa d \alpha
$$

We get

$$
\begin{aligned}
\int \vartheta^{2}(\alpha)\left|\left[f_{h}\right]_{h}(\alpha)\right|^{3} d \alpha \\
\leq 20\left(\iint_{I}^{\infty}\left|\left[f_{\kappa}\right]_{\kappa}(\alpha)\right|^{2} d \kappa d \alpha\right)^{1 / 2} \\
\quad \cdot \iint_{h}^{\infty}\left(\left|(\vartheta f)_{\kappa \kappa}(\alpha)\right|^{2}+\left|\frac{d}{d \kappa}\left[(\vartheta f)_{\kappa}\right]_{\kappa}(\alpha)\right|^{2}\right) d \kappa d \alpha \\
\quad+c_{3}|I|^{2}\left(\left.\left.\int|| D\right|^{1 / 2}(\sigma f)(\alpha)\right|^{2} d \alpha\right)^{3 / 2}+c_{4}|I|^{1 / 2}\left(\int_{0}^{A}|f(\alpha)|^{2} d \alpha\right)^{3 / 2},
\end{aligned}
$$

where $c_{3}$ and $c_{4}$ are universal constants.

We estimate $|I| \int \vartheta(\alpha)\left|\left[f_{h}\right]_{h}(\alpha)\right|^{2} d \alpha$ for $f=Z_{\alpha}$ or $f=\ln Z_{\alpha}$ similarly. We have

$$
\begin{aligned}
&|I| \int \vartheta(\alpha)\left|\left[f_{h}\right] h(\alpha)\right|^{2} d \alpha \\
& \leq 2|I| \iint_{h}^{\infty} \vartheta(\alpha)\left|\left[f_{\kappa}\right]_{\kappa}(\alpha)\right| \frac{d}{d \kappa}\left[f_{\kappa}\right]_{\kappa}(\alpha) \mid d \kappa d \alpha \\
& \leq 2|I|\left(\iint_{I}^{\infty}\left|\left[f_{\kappa}\right]_{\kappa}(\alpha)\right|^{2} d \kappa d \alpha\right)^{1 / 2} \\
& \cdot\left(\iint_{h}^{\infty} \vartheta^{2}(\alpha)\left|\frac{d}{d \kappa}\left[f_{\kappa}\right]_{\kappa}(\alpha)\right|^{2} d \kappa d \alpha\right)^{1 / 2} \\
& \leq\left(\iint_{I}^{\infty}\left|\left[f_{\kappa}\right]_{\kappa}(\alpha)\right|^{2} d \kappa d \alpha\right)^{1 / 2}\left(\iint_{h}^{\infty} \vartheta^{2}(\alpha)\left|\frac{d}{d \kappa}\left[f_{\kappa}\right]_{\kappa}(\alpha)\right|^{2} d \kappa d \alpha\right) \\
&+|I|^{2}\left(\iint_{0}^{\infty}\left|\left[f_{\kappa}\right]_{\kappa}(\alpha)\right|^{2} d \kappa d \alpha\right)^{1 / 2} \\
& \leq 3\left(\iint_{I}^{\infty}\left|\left[f_{\kappa}\right]_{\kappa}(\alpha)\right|^{2} d \kappa d \alpha\right)^{1 / 2} \iint_{h}^{\infty}\left|\frac{d}{d \kappa}\left[(\vartheta f)_{\kappa}\right]_{\kappa}(\alpha)\right|^{2} d \kappa d \alpha \\
&+c_{5}|I|^{2}\left(\left.\left.\int|| D\right|^{1 / 2}(\sigma f)(\alpha)\right|^{2} d \alpha\right)^{3 / 2}+c_{6}|I|^{1 / 2}\left(\int_{0}^{A}|f(\alpha)|^{2} d \alpha\right)^{3 / 2} \\
&+c_{7}|I|^{2}\left(\left.\left.\int|| D\right|^{1 / 2}(\sigma f)(\alpha)\right|^{2} d \alpha\right)^{1 / 2}+c_{8}|I|^{3 / 2}\left(\int_{0}^{A}|f(\alpha)|^{2} d \alpha\right)^{1 / 2} .
\end{aligned}
$$


Let's sum up our estimates so far. We note that for $0<|l| \leq \frac{1}{16} \delta_{1}$,

$$
\begin{aligned}
\frac{1}{l^{2}} & \int_{0}^{l} h \iint \frac{1}{(\alpha-\beta)^{2}}\left|\vartheta(\alpha)\left(\left[\ln z_{\alpha}\right]_{h}\right)_{h}(\alpha, 0)-\vartheta(\beta)\left(\left[\ln z_{\beta}\right]_{h}\right)_{h}(\beta, 0)\right|^{2} d \alpha d \beta d h \\
& =\left.\left.\frac{2 \pi}{l^{2}} \int_{0}^{l} h \int|| D\right|^{1 / 2}\left(\vartheta(\alpha)\left(\left[\ln z_{\alpha}\right]_{h}\right)_{h}(\alpha, 0)\right)\right|^{2} d \alpha d h<\infty
\end{aligned}
$$

This is an easy consequence of Lemma 4.7 given a little later.

From (4.55), using the estimates in (4.56) through (4.74), the Fourier transform, and Plancherel's theorem, we obtain, for $0<|l| \leq \frac{1}{16} \delta_{1}$,

$$
\begin{aligned}
\frac{1}{36 l^{2}} \int_{0}^{l} h \int \zeta(t) \int \vartheta^{2}(\alpha)\left|z_{t h h}(\alpha, t)\right|^{2} d \alpha d t d h \\
+\frac{v}{36 l^{2}} \int_{0}^{l} h \int \zeta(t) \int \vartheta^{2}(\alpha)\left|z_{\alpha h h}(\alpha, t)\right|^{2} d \alpha d t d h \\
+\left.\left.\frac{T_{1}^{2}}{l^{2}} \int_{0}^{l} h \int|| D\right|^{1 / 2}\left(\vartheta(\alpha)\left(\left[\ln z_{\alpha}\right]_{h}\right)_{h}(\alpha, 0)\right)\right|^{2} d \alpha d h \\
\leq K_{2}+\left.\left.C_{9}\left\|\ln z_{\alpha}(\cdot, 0)\right\|_{*, \delta_{0}} \frac{T_{1}^{2}}{l^{2}} \int_{0}^{l} h \int|| D\right|^{1 / 2}\left[\left(\vartheta z_{\alpha}\right)_{h}\right]_{h}(\alpha, 0)\right|^{2} d \alpha d h \\
+C_{10}\left(\iint_{I}^{\infty}\left|Z_{h h}(\alpha)\right|^{2} d h d \alpha\right)^{1 / 2} \\
\left.\left.\quad \cdot \frac{T_{1}^{2}}{l^{2}} \int_{0}^{l} h \int|| D\right|^{1 / 2}\left[\left(\vartheta z_{\alpha}\right)_{h}\right]_{h}(\alpha, 0)\right|^{2} d \alpha d h \\
+\left.\left.C_{11}\left\|\ln z_{\alpha}(\cdot, 0)\right\|_{*, \delta_{0}} \frac{T_{1}^{2}}{l^{2}} \int_{0}^{l} h \int|| D\right|^{1 / 2}\left(\left(\left[\vartheta \ln z_{\alpha}\right]_{h}\right)_{h}(\alpha, 0)\right)\right|^{2} d \alpha d h \\
+C_{12}\left(\iint_{I}^{\infty}\left|\left(\left[\ln Z_{\alpha}\right]_{h}\right)_{h}(\alpha)\right|^{2} d h d \alpha\right)^{1 / 2} \\
\left.\left.\quad \cdot \frac{T_{1}^{2}}{l^{2}} \int_{0}^{l} h \int|| D\right|^{1 / 2}\left(\left(\left[\vartheta \ln z_{\alpha}\right]_{h}\right)_{h}(\alpha, 0)\right)\right|^{2} d \alpha d h,
\end{aligned}
$$

where $C_{9}, C_{10}, C_{11}$, and $C_{12}$ are constants depending on $m$ and $M$ only, and $K_{2}<$ $\infty$ is independent of $l$.

We now handle the left-hand side of inequality (4.75). First, we have

$$
\begin{aligned}
& \left.\left.T_{1}^{2} \int|| D\right|^{1 / 2}\left(\vartheta(\alpha) z_{h h}(\alpha, 0)\right)\right|^{2} d \alpha \\
& \leq \frac{1}{\sqrt{v}}\left(\int \zeta(t) \int \vartheta^{2}(\alpha)\left|z_{t h h}(\alpha, t)\right|^{2} d \alpha d t\right. \\
& \left.\quad+v \int \zeta(t) \int \vartheta^{2}(\alpha)\left|z_{\alpha h h}(\alpha, t)\right|^{2} d \alpha d t\right) \\
& \quad+c_{9} \int_{0}^{T_{1}} \int_{c-\frac{1}{2} \delta_{1}}^{d+\frac{1}{2} \delta_{1}}\left|z_{\alpha \alpha}(\alpha, t)\right|^{2} d \alpha d t .
\end{aligned}
$$


This can be obtained as in (3.47). Here $c_{9}$ is a constant depending on $|I|, T_{1}, m$, and $M$. The following lemma gives an estimate for the commutator $\vartheta\left[f_{h}\right]_{h}(\alpha)-$ $\left[(\vartheta f)_{h}\right]_{h}(\alpha)$ :

Lemma 4.7 Assume that $\vartheta \in C^{1,1}(\mathbb{R})$, supp $\vartheta \subset I=[p, q]$, and $\varrho=\varrho(\alpha)$ is defined in (4.63). Then there exist constants $c_{10}$ and $c_{11}$ such that for any $f \in$ $H_{\text {loc }}^{1 / 2}(\mathbb{R}), 0<|h| \leq \frac{1}{16} \delta_{1}$,

$$
\begin{aligned}
& \left.\left.\int|| D\right|^{1 / 2}\left(\vartheta(\alpha)\left[f_{h}\right]_{h}(\alpha)-\left[(\vartheta f)_{h}\right]_{h}\right)(\alpha)\right|^{2} d \alpha \\
& \leq\left.\left. c_{1}\left\|\vartheta^{\prime}\right\|_{\infty}^{2} \int|| D\right|^{1 / 2}(\varrho f)(\alpha)\right|^{2} d \alpha \\
& \quad+c_{2}\left(\left\|\vartheta^{\prime \prime}\right\|_{\infty}^{2}\left(|I|+2 \delta_{1}\right)+\frac{1}{|I|+2 \delta_{1}}\left\|\vartheta^{\prime}\right\|_{\infty}^{2}\right) \int|\varrho(\alpha) f(\alpha)|^{2} d \alpha
\end{aligned}
$$

Lemma 4.7 is an easy consequence of (4.69); we omit the proof. We apply (4.76) and Lemma 4.7 to the left-hand side of (4.75) and obtain, for $0<|l| \leq \frac{1}{16} \delta_{1}$,

$$
\begin{aligned}
&\left.\left.\frac{T_{1}^{2} \sqrt{v}}{72 l^{2}} \int_{0}^{l} h \int|| D\right|^{1 / 2}\left[\left(\vartheta z_{\alpha}\right)_{h}\right]_{h}(\alpha, 0)\right|^{2} d \alpha d h \\
&+\left.\left.\frac{T_{1}^{2}}{2 l^{2}} \int_{0}^{l} h \int|| D\right|^{1 / 2}\left(\left[\vartheta \ln z_{\alpha}\right]_{h}\right)_{h}(\alpha, 0)\right|^{2} d \alpha d h \\
& \leq\left.\left.C_{9}\left\|\ln z_{\alpha}(\cdot, 0)\right\|_{*, \delta_{0}} \frac{T_{1}^{2}}{l^{2}} \int_{0}^{l} h \int|| D\right|^{1 / 2}\left[\left(\vartheta z_{\alpha}\right)_{h}\right]_{h}(\alpha, 0)\right|^{2} d \alpha d h \\
&+ C_{10}\left(\iint_{I}^{\infty}\left|Z_{h h}(\alpha)\right|^{2} d h d \alpha\right)^{1 / 2} \\
&\left.\left.\cdot \frac{T_{1}^{2}}{l^{2}} \int_{0}^{l} h \int|| D\right|^{1 / 2}\left[\left(\vartheta z_{\alpha}\right)_{h}\right]_{h}(\alpha, 0)\right|^{2} d \alpha d h \\
&+\left.\left.C_{11}\left\|\ln z_{\alpha}(\cdot, 0)\right\|_{*, \delta_{0}} \frac{T_{1}^{2}}{l^{2}} \int_{0}^{l} h \int|| D\right|^{1 / 2}\left(\left(\left[\vartheta \ln Z_{\alpha}\right]_{h}\right)_{h}(\alpha, 0)\right)\right|^{2} d \alpha d h \\
&+ C_{12}\left(\iint_{I}^{\infty}\left|\left(\left[\ln Z_{\alpha}\right]_{h}\right)_{h}(\alpha)\right|^{2} d h d \alpha\right)^{1 / 2} \\
&\left.\left.\cdot \frac{T_{1}^{2}}{l^{2}} \int_{0}^{l} h \int|| D\right|^{1 / 2}\left(\left(\left[\vartheta \ln z_{\alpha}\right]_{h}\right)_{h}(\alpha, 0)\right)\right|^{2} d \alpha d h+K_{3} .
\end{aligned}
$$

Here $K_{3}<\infty$ is independent of $l$. Since $Z_{\alpha} \in H_{\text {loc }}^{1 / 2}(\mathbb{R})$ and $\ln Z_{\alpha} \in H_{\text {loc }}^{1 / 2}(\mathbb{R})$, we have, from Lemma 4.6, that for any $\epsilon>0$, there is an $\iota$ such that whenever $|I| \leq \iota$ and $I \subset[c, d]$,

$$
\iint_{I}^{\infty}\left|Z_{h h}(\alpha)\right|^{2} d h d \alpha+\iint_{I}^{\infty}\left|\left(\left[\ln Z_{\alpha}\right]_{h}\right)_{h}(\alpha)\right|^{2} d h d \alpha \leq \epsilon .
$$


Now take $I \subset[c, d]$ small enough such that

$$
C_{10}\left(\iint_{I}^{\infty}\left|Z_{h h}(\alpha)\right|^{2} d h d \alpha\right)^{1 / 2} \leq \frac{\sqrt{v}}{288}
$$

and

$$
C_{12}\left(\iint_{I}^{\infty}\left|\left(\left[\ln Z_{\alpha}\right]_{h}\right)_{h}(\alpha)\right|^{2} d h d \alpha\right)^{1 / 2} \leq \frac{1}{8} .
$$

We have, whenever

$$
\sup _{[0, T]}\left\|\ln z_{\alpha}(\cdot, t)\right\|_{\mathrm{BMO}(a, b), \delta_{0}} \leq \min \left\{c_{1}(m, M), \frac{\sqrt{v}}{288 C_{9}}, \frac{1}{8 C_{11}}\right\},
$$

where $c_{1}(m, M)$ is as in (4.45),

$$
\begin{aligned}
& \left.\left.\frac{T_{1}^{2} \sqrt{v}}{144 l^{2}} \int_{0}^{l} h \int|| D\right|^{1 / 2}\left[\left(\vartheta z_{\alpha}\right)_{h}\right]_{h}(\alpha, 0)\right|^{2} d \alpha d h \\
& +\left.\left.\frac{T_{1}^{2}}{4 l^{2}} \int_{0}^{l} h \int|| D\right|^{1 / 2}\left(\left[\vartheta \ln z_{\alpha}\right]_{h}\right)_{h}(\alpha, 0)\right|^{2} d \alpha d h<K_{3} .
\end{aligned}
$$

Let $l \rightarrow 0$. Since the small interval $I \subset[c, d] \subset(a, b)$ is arbitrary, we get $z_{\alpha}(\cdot, 0) \in H_{\mathrm{loc}}^{3 / 2}(a, b)$ and $\ln z_{\alpha}(\cdot, 0) \in H_{\mathrm{loc}}^{3 / 2}(a, b)$. The fact that $z_{\alpha \alpha} \in H^{1}(K)$ for $K$ compact in $(a, b) \times\left[0, T_{1}\right)$ follows from (4.75) and (4.79) by taking $l \rightarrow 0$. The fact that $z_{\alpha} \in C((a, b) \times[0, T))$ follows from Sobolev embedding, and $z_{\alpha} \in$ $H_{\text {loc }}^{2}((a, b) \times[0, T))$ follows from taking derivatives $\partial_{t} \partial_{\alpha}$ of equation (1.6). This completes the proof of Proposition 4.1.

\section{$5 C^{\infty}$ Regularity of $z_{\alpha}$ and $C^{\infty}$ Compatibility of the Initial Data}

From now on we can assume in addition to the assumptions in Theorem 1.2, in particular assumption (i), that $z_{\alpha}, z_{\alpha \alpha} \in H_{\mathrm{loc}}^{1}((a, b) \times(0, T))$. We show in this section that a solution $z=z(\alpha, t)$ of the Birkhoff-Rott equation (1.6), under the assumptions of Theorem 1.2, is in $C^{\infty}(a, b)$ for each fixed $t \in(0, T)$. In fact, $z \in C^{\infty}((a, b) \times(0, T))$. We will also prove the $C^{\infty}$ compatibility of the initial data.

\section{PROPOSITION 5.1}

(i) Assume $z \in H^{1}\left([0, T], L_{\text {loc }}^{2}(\mathbb{R})\right) \cap L^{2}\left([0, T], H_{\text {loc }}^{1}(\mathbb{R})\right)$ is a solution of the Birkhoff-Rott equation (1.6) for $0 \leq t \leq T$, satisfying assumption (i) of Theorem 1.2. Assume that $z_{\alpha}, \partial_{\alpha} z_{\alpha} \in H_{\mathrm{loc}}^{1}((a, b) \times(0, T))$. Then $\partial_{\alpha}^{j} z_{\alpha} \in H_{\mathrm{loc}}^{1}((a, b) \times$ $(0, T))$ for all $j \geq 2$.

(ii) Let $j \geq 2$ be fixed. If, in addition,

$$
z_{\alpha}, \partial_{\alpha} z_{\alpha} \in H_{\mathrm{loc}}^{1}((a, b) \times[0, T)) \quad \text { and } \operatorname{Im}\left((1+i) \ln z_{\alpha}(\cdot, 0)\right) \in H_{\mathrm{loc}}^{j+1 / 2}(a, b) \text {, }
$$


then

$$
\begin{gathered}
\operatorname{Re}\left((1+i) \ln z_{\alpha}(\cdot, 0)\right) \in H_{\mathrm{loc}}^{j+1 / 2}(a, b), \quad z_{\alpha}(\cdot, 0) \in H_{\mathrm{loc}}^{j+1 / 2}(a, b), \\
\text { and } \partial_{\alpha}^{j} z_{\alpha} \in H_{\mathrm{loc}}^{1}((a, b) \times[0, T)) .
\end{gathered}
$$

Remark. Although we don't state it explicitly in Proposition 5.1, it is not difficult to see that we can obtain $z_{\alpha} \in H_{\mathrm{loc}}^{j+1}((a, b) \times(0, T))$ by taking derivatives on both sides of (1.6) with respect to $\alpha$ and $t$.

A corollary of Proposition 5.1 is that for each fixed $t \in(0, T), z_{\alpha}(\cdot, t) \in$ $C^{\infty}(a, b)$.

We will prove Proposition 5.1 by induction. Before we proceed, we need some lemmas.

Let $\left(\partial_{\beta} \varrho(\beta)\right)^{j} f(\beta)=\partial_{\beta} \varrho \cdots \partial_{\beta} \varrho f(\beta)$ and $\left(\varrho(\alpha) \partial_{\alpha}\right)^{j} f(\alpha)=\varrho \partial_{\alpha} \cdots \varrho \partial_{\alpha} f(\alpha)$, that is, $\partial_{\beta} \varrho(\beta)$ is applied to $f(\beta) j$ times and $\varrho(\alpha) \partial_{\alpha}$ is applied to $f(\alpha) j$ times.

Lemma 5.2 Assume that $K \in C^{\infty}\left(\mathbb{R}^{2} \backslash \Delta\right)$, where $\Delta=\{(\alpha, \alpha): \alpha \in \mathbb{R}\}$, $K(\alpha, \beta)=-K(\beta, \alpha)$, and for each $j \geq 0$, there exist constants $C_{j}$ such that for all $(\alpha, \beta) \in \mathbb{R}^{2} \backslash \Delta,\left|\partial_{\alpha}^{j} K(\alpha, \beta)\right| \leq \frac{C_{j}}{|\alpha-\beta|^{j+1}}$. Let $\varrho, f \in C^{\infty}(\mathbb{R})$, and let $\varrho$ or $f$ be compactly supported. Then

$$
\begin{aligned}
& \varrho(\alpha) \partial_{\alpha} \int K(\alpha, \beta) f(\beta) d \beta \\
& =\int\left(\varrho(\alpha) \partial_{\alpha}+\varrho(\beta) \partial_{\beta}\right) K(\alpha, \beta) f(\beta) d \beta+\int K(\alpha, \beta) \partial_{\beta}(\varrho f)(\beta) d \beta
\end{aligned}
$$

and

$$
\begin{aligned}
\left(\varrho(\alpha) \partial_{\alpha}\right)^{j} & \int K(\alpha, \beta) f(\beta) d \beta \\
& =\sum_{l=0}^{j}\left(\begin{array}{l}
l \\
j
\end{array}\right) \int\left(\varrho(\alpha) \partial_{\alpha}+\varrho(\beta) \partial_{\beta}\right)^{l} K(\alpha, \beta)\left(\partial_{\beta} \varrho(\beta)\right)^{j-l} f(\beta) d \beta .
\end{aligned}
$$

Here $\left(\begin{array}{l}l \\ j\end{array}\right)=\frac{j !}{l !(j-l) !}$.

In (5.1) and (5.2), the integrals are in the sense of principal value. Equality (5.1) is an easy consequence of integration by parts and distribution theory, while (5.2) directly follows from (5.1) by induction.

We are mainly interested in applying Lemma 5.2 to

$$
K(\alpha, \beta)=\frac{1}{z(\alpha, t)-z(\beta, t)}
$$

for fixed $t \in(0, T)$, where $z=z(\alpha, t)$ is a solution of the Birkhoff-Rott equation (1.6). 
Lemma 5.3 Let $\varrho, z \in C^{\infty}(\mathbb{R})$. Assume that $z(\alpha)-z(\beta) \neq 0$ for $\alpha \neq \beta$. Then for $j \geq 1$,

$$
\text { (5.3) } \begin{aligned}
& \frac{1}{j !}\left(\varrho(\alpha) \partial_{\alpha}+\varrho(\beta) \partial_{\beta}\right)^{j} \frac{1}{z(\alpha)-z(\beta)} \\
= & \sum_{n=1}^{j}(-1)^{n} \frac{1}{(z(\alpha)-z(\beta))^{n+1}} \sum_{\substack{k_{1}+\cdots+k_{n}=j \\
k_{i} \geq 1}} \frac{\prod_{i=1}^{n}\left(\left(\varrho(\alpha) \partial_{\alpha}\right)^{k_{i}} z(\alpha)-\left(\varrho(\beta) \partial_{\beta}\right)^{k_{i}} z(\beta)\right)}{k_{1} ! \cdots k_{n} !} .
\end{aligned}
$$

Equality (5.3) is inspired by the fact that

$$
\frac{1}{j !}\left(\frac{1}{g}\right)^{(j)}(0)=\sum_{n=1}^{j}(-1)^{n} \frac{1}{g(0)^{n+1}} \sum_{\substack{k_{1}+\cdots+k_{n}=j \\ k_{i} \geq 1}} \frac{g^{\left(k_{1}\right)}(0) \cdots g^{\left(k_{n}\right)}(0)}{k_{1} ! \cdots k_{n} !},
$$

which in turn can be proved using Taylor expansion. Equality (5.3) can be proved directly by induction. We omit the proof.

LEMmA 5.4 Assume that $f \in H^{1}(I)$, where $I \subset \mathbb{R}$ is a bounded, closed interval. Then

$$
\|f\|_{L^{\infty}(I)} \leq|I|^{-1 / 2}\|f\|_{L^{2}(I)}+|I|^{1 / 2}\left\|f^{\prime}\right\|_{L^{2}(I)} .
$$

If in addition $f\left(x_{0}\right)=0$ for some $x_{0} \in I$, then

$$
\|f\|_{L^{\infty}(I)} \leq|I|^{1 / 2}\left\|f^{\prime}\right\|_{L^{2}(I)} .
$$

Lemma 5.4 is just a special case of the Sobolev embedding theorem. We define

$$
\|\widetilde{f f}\|_{H^{1}(I)}=|I|^{-1 / 2}\|f\|_{L^{2}(I)}+|I|^{1 / 2}\left\|f^{\prime}\right\|_{L^{2}(I)} .
$$

The following is a generalization of Corollary 2.5. It is a consequence of the $\mathrm{Tb}$ theorem (see $[10,19]$ for a statement of the $\mathrm{Tb}$ theorem).

Let $z: \mathbb{R} \rightarrow \mathbb{C}$ be a complex-valued function satisfying

$$
m|\alpha-\beta| \leq|z(\alpha)-z(\beta)| \leq M|\alpha-\beta| \quad \text { for all } \alpha, \beta \in \mathbb{R}
$$

for some constants $m, M>0$. Let

$$
\begin{aligned}
B_{k}\left(A_{1}, \ldots, A_{k}, f\right) & (\alpha) \\
& =\int \frac{\left(A_{1}(\alpha)-A_{1}(\beta)\right) \cdots\left(A_{k}(\alpha)-A_{k}(\beta)\right)}{(z(\alpha)-z(\beta))^{k+1}} f(\beta) d \beta .
\end{aligned}
$$

LEMMA 5.5 There exists a constant $C>0$ such that for all $A_{i}$, with $A_{i}^{\prime} \in L^{\infty}(\mathbb{R})$, $f \in L^{2}(\mathbb{R})$, and nonnegative integer $k$,

$$
\left\|B_{k}\left(A_{1}, \ldots, A_{k}, f\right)\right\|_{L^{2}(\mathbb{R})} \leq C^{k+1}\left\|A_{1}^{\prime}\right\|_{L^{\infty}(\mathbb{R})} \cdots\left\|A_{k}^{\prime}\right\|_{L^{\infty}(\mathbb{R})}\|f\|_{L^{2}(\mathbb{R})} .
$$

For the multilinear operator $B_{k}\left(A_{1}, \ldots, A_{k}, f\right)$ we will also need the following estimate:

Lemma 5.6 Let $I \subset \mathbb{R}$ be a bounded interval. Assume that $z: \mathbb{R} \rightarrow \mathbb{C}$ satisfies (5.6), $z_{\alpha} \in H^{1}(I)$, and $f \in H_{0}^{1}(I)$, supp $f \subset I$. 
(i) Assume $A_{1} \in H^{1}(I)$ and $A_{i} \in H^{2}(I)$ for $2 \leq i \leq k$. Then there exists $a$ constant $C_{0}>0$, depending on $m, M$, and $|I|$, such that

$$
\begin{aligned}
& \left\|B_{k}\left(A_{1}, \ldots, A_{k}, f\right)\right\|_{L^{2}(I)} \\
& \quad \leq C_{0}^{k}\left(1+\left\|z_{\alpha \alpha}\right\|_{L^{2}(I)}\right)^{k}\left\|A_{1}\right\|_{H^{1}(I)}\left\|A_{2}\right\|_{H^{2}(I)} \cdots\left\|A_{k}\right\|_{H^{2}(I)}\left\|f^{\prime}\right\|_{L^{2}(I)} .
\end{aligned}
$$

(ii) Assume that $A_{1} \in H_{0}^{1}(I)$ and $A_{i} \in H_{0}^{2}(I)$ for $2 \leq i \leq k$, and $\operatorname{supp} A_{j} \subset I$ for $1 \leq j \leq k$. Then

$$
\begin{aligned}
& \left\|B_{k}\left(A_{1}, \ldots, A_{k}, f\right)\right\|_{L^{2}(\mathbb{R})} \\
& \quad \leq C_{0}^{k}\left(1+\left\|z_{\alpha \alpha}\right\|_{L^{2}(I)}\right)^{k}\left\|A_{1}^{\prime}\right\|_{L^{2}(I)}\left\|A_{2}^{\prime \prime}\right\|_{L^{2}(I)} \cdots\left\|A_{k}^{\prime \prime}\right\|_{L^{2}(I)}\left\|f^{\prime}\right\|_{L^{2}(I)} .
\end{aligned}
$$

Remark 5.7. Under the assumption of Lemma 5.6(ii), we have

$$
\begin{aligned}
& \left\|B_{k}\left(A_{1}, \ldots, A_{k}, 1\right)\right\|_{L^{2}(\mathbb{R})} \\
& \quad \leq C_{0}^{k}\left(1+\left\|z_{\alpha \alpha}\right\|_{L^{2}(I)}\right)^{k}\left\|A_{1}^{\prime}\right\|_{L^{2}(I)}\left\|A_{2}^{\prime \prime}\right\|_{L^{2}(I)} \cdots\left\|A_{k}^{\prime \prime}\right\|_{L^{2}(I)} .
\end{aligned}
$$

Inequality (5.11) can be proved by taking a function $\rho \in C_{0}^{\infty}(\mathbb{R})$ such that $\rho(\alpha)=$ 1 for $\alpha \in 2 I$ and decomposing

$$
B_{k}\left(A_{1}, \ldots, A_{k}, 1\right)=B_{k}\left(A_{1}, \ldots, A_{k}, \rho\right)+B_{k}\left(A_{1}, \ldots, A_{k}, 1-\rho\right) .
$$

Remark 5.8. In the proof, a more careful account of the role of $|I|$ leads to the following inequality under the assumptions of Lemma 5.6(i):

$$
\begin{aligned}
& \left\|B_{k}\left(A_{1}, \ldots, A_{k}, f\right)\right\|_{L^{2}(I)} \\
& \quad \leq C_{0}^{\prime k}\left(1+|I|^{1 / 2}\left\|z_{\alpha \alpha}\right\|_{L^{2}(I)}\right)^{k} \widetilde{\left\|A_{1}\right\|_{H^{1}(I)}} \prod_{j=2}^{k} \widetilde{\left\|A_{j}^{\prime}\right\|_{H^{1}(I)}}\left\|f^{\prime}\right\|_{L^{2}(I)}
\end{aligned}
$$

where $C_{0}^{\prime}$ depends on $m$ and $M$ only.

Proof: The following proof applies to both (5.9) and (5.10). However, we will write only in terms of (5.9). We will use Lemma 5.5. Notice that Lemma 5.5 is given on the whole line $\mathbb{R}$, while (5.9) is an inequality on an interval $I$. In order to apply (5.8) to prove (5.9), we need to reflect and then periodically extend the functions $A_{i}$ to $\mathbb{R}$, and extend $f$ to $\mathbb{R}$ by taking $f=0$ outside the interval $I$.

We use integration by parts and induction. For $k=1$,

$$
\begin{aligned}
B_{1}\left(A_{1}, f\right)= & \int \frac{\left(A_{1}(\alpha)-A_{1}(\beta)\right)}{(z(\alpha)-z(\beta))^{2}} f(\beta) d \beta \\
= & -\int \frac{A_{1}(\alpha)-A_{1}(\beta)}{z(\alpha)-z(\beta)} \partial_{\beta}\left(f(\beta) \frac{1}{z_{\beta}(\beta)}\right) d \beta \\
& +\int \frac{1}{z(\alpha)-z(\beta)} A_{1}^{\prime}(\beta) f(\beta) \frac{1}{z_{\beta}(\beta)} d \beta
\end{aligned}
$$


Applying (5.8) with $k=0$, we get, for $f \in H_{0}^{1}(I)$ and supp $f \subset I$,

$$
\begin{aligned}
\left\|B_{1}\left(A_{1}, f\right)\right\|_{L^{2}(I)} & \leq\left\|B_{1}\left(A_{1}, f\right)\right\|_{L^{2}(\mathbb{R})} \\
& \leq C\left(\left\|A_{1}^{\prime} f \frac{1}{z_{\beta}}\right\|_{L^{2}(I)}+2\left\|A_{1}\right\|_{L^{\infty}(I)}\left\|\partial_{\beta}\left(f \frac{1}{z_{\beta}}\right)\right\|_{L^{2}(I)}\right) .
\end{aligned}
$$

Therefore it follows from Lemma 5.4 and assumption (5.6) that

$$
\left\|B_{1}\left(A_{1}, f\right)\right\|_{L^{2}(I)} \leq C_{0}\left(1+\left\|z_{\alpha \alpha}\right\|_{L^{2}(I)}\right)\left\|A_{1}\right\|_{H^{1}(I)}\left\|f^{\prime}\right\|_{L^{2}(I)}
$$

for some constant $C_{0}$ depending on $C, m$, and $|I|$.

Assume that (5.9) holds for $k=l-1$; we now prove for $k=l$. From integration by parts, we have

$$
\begin{aligned}
B_{l}( & \left.A_{1}, \ldots, A_{l}, f\right) \\
= & \int \frac{\left(A_{1}(\alpha)-A_{1}(\beta)\right) \cdots\left(A_{l}(\alpha)-A_{l}(\beta)\right)}{(z(\alpha)-z(\beta))^{l+1}} f(\beta) d \beta \\
= & \frac{1}{l} \int \frac{\prod_{j=2}^{l}\left(A_{j}(\alpha)-A_{j}(\beta)\right)}{(z(\alpha)-z(\beta))^{l}} A_{1}^{\prime}(\beta) f(\beta) \frac{1}{z_{\beta}(\beta)} d \beta \\
& +\frac{1}{l} \sum_{i=2}^{l} \int \frac{\prod_{j \neq i}\left(A_{j}(\alpha)-A_{j}(\beta)\right)}{(z(\alpha)-z(\beta))^{l}} A_{i}^{\prime}(\beta) f(\beta) \frac{1}{z_{\beta}(\beta)} d \beta \\
& -\frac{1}{l} \int \frac{\left(A_{1}(\alpha)-A_{1}(\beta)\right) \cdots\left(A_{l}(\alpha)-A_{l}(\beta)\right)}{(z(\alpha)-z(\beta))^{l}} \partial_{\beta}\left(f(\beta) \frac{1}{z_{\beta}(\beta)}\right) d \beta .
\end{aligned}
$$

We apply Lemma 5.5 to the first and third terms and the induction hypothesis to the second term. We get

$$
\begin{aligned}
&\left\|B_{l}\left(A_{1}, \ldots, A_{l}, f\right)\right\|_{L^{2}(I)} \\
& \leq \frac{1}{l} C^{l}\left\|A_{2}^{\prime}\right\|_{L^{\infty}(I)} \cdots\left\|A_{l}^{\prime}\right\|_{L^{\infty}(I)}\left\|A_{1}^{\prime} f \frac{1}{z_{\alpha}}\right\|_{L^{2}(I)} \\
&+\frac{1}{l} \sum_{i=2}^{l} C_{0}^{l-1}\left(1+\left\|z_{\alpha \alpha}\right\|_{L^{2}(I)}\right)^{l-1}\left\|A_{1}\right\|_{H^{1}(I)} \prod_{j \neq i, j \neq 1}\left\|A_{j}\right\|_{H^{2}(I)}\left\|\partial_{\alpha}\left(A_{i}^{\prime} f \frac{1}{z_{\alpha}}\right)\right\|_{L^{2}(I)} \\
&+\frac{2}{l} C^{l}\left\|A_{1}\right\|_{L^{\infty}(I)}\left\|A_{2}^{\prime}\right\|_{L^{\infty}(I)} \cdots\left\|A_{l}^{\prime}\right\|_{L^{\infty}(I)}\left\|\partial_{\alpha}\left(f \frac{1}{z_{\alpha}}\right)\right\|_{L^{2}(I)} .
\end{aligned}
$$

From here and Lemma 5.4 we get (5.9) for $k=l$.

Lemma 5.9 Assume that $A_{1} \in H^{1 / 2}(\mathbb{R}), A_{i} \in H^{3 / 2}(\mathbb{R})$ for $2 \leq i \leq k$, and $A_{j}$, $1 \leq j \leq k$, are compactly supported. Then there exists a constant $C_{1}$ such that

$$
\begin{aligned}
\||D|^{1 / 2}\left(A_{1}\right. & \left.\cdots A_{k}\right) \|_{L^{2}(\mathbb{R})} \\
& \leq C_{1}^{k-1}\left\||D|^{1 / 2} A_{1}\right\|_{L^{2}(\mathbb{R})}\left\||D|^{1 / 2} A_{2}^{\prime}\right\|_{L^{2}(\mathbb{R})} \cdots\left\||D|^{1 / 2} A_{k}^{\prime}\right\|_{L^{2}(\mathbb{R})} .
\end{aligned}
$$


For $k=2,(5.12)$ can be proved using some easy Fourier analysis and Poincare's inequality. For $k>2,(5.12)$ can be obtained by induction. We omit the details.

We are now ready to prove Proposition 5.1. We will treat the case $j=2$ first, then give a unified proof for all $j \geq 3$. We will again use the difference quotient argument. However, the proof will be easier from now on because we have some better regularity $z_{\alpha}, z_{\alpha \alpha} \in H_{\mathrm{loc}}^{1}((a, b) \times(0, T))$ to begin with.

\section{1 $\boldsymbol{H}^{\mathbf{1}}$ Regularity of $\boldsymbol{\partial}_{\alpha}^{2} z_{\alpha}$ and $\boldsymbol{H}^{\mathbf{5} / \mathbf{2}}$ Compatibility of the Initial Data}

Let $Q$ and $\mathcal{T}$ be compact intervals, and $3 Q \subset(a, b)$ and $\mathcal{T} \subset(0, T)$. Take $\eta, \rho \in C_{0}^{\infty}(\mathbb{R})$ such that

$$
\operatorname{supp} \eta \subset Q, \quad \rho(\alpha)=1 \text { on } \frac{3}{2} Q, \quad \operatorname{supp} \rho \subset 2 Q,
$$

and $0 \leq \eta(\alpha), \rho(\alpha) \leq 1,\left|\eta^{\prime}(\alpha)\right|+\left|\rho^{\prime}(\alpha)\right| \leq C_{2} /|Q|$, for some constant $C_{2}>0$. Taking derivatives $\partial_{\alpha}^{2}$ on both sides of (1.6), we have from Lemmas 5.2 and 5.3, distribution theory, and the assumption $z_{\alpha}, z_{\alpha \alpha} \in H_{\mathrm{loc}}^{1}((a, b) \times(0, T))$ that for any $\alpha \in Q$,

$$
\begin{aligned}
\bar{z}_{t \alpha \alpha}(\alpha, t)= & \frac{1}{2 \pi i} \int \frac{-z_{\alpha \alpha}(\alpha, t)+z_{\beta \beta}(\beta, t)}{(z(\alpha, t)-z(\beta, t))^{2}} \rho(\beta) d \beta \\
& +\frac{1}{\pi i} \int \frac{\left(z_{\alpha}(\alpha, t)-z_{\beta}(\beta, t)\right)^{2}}{(z(\alpha, t)-z(\beta, t))^{3}} \rho(\beta) d \beta+f_{1}(\alpha, t),
\end{aligned}
$$

where

$$
\begin{aligned}
& f_{1}(\alpha, t) \\
& =\frac{1}{\pi i} \int \frac{-z_{\alpha}(\alpha, t)+z_{\beta}(\beta, t)}{(z(\alpha, t)-z(\beta, t))^{2}} \rho^{\prime}(\beta) d \beta+\frac{1}{2 \pi i} \int \frac{1}{z(\alpha, t)-z(\beta, t)} \rho^{\prime \prime}(\beta) d \beta \\
& \quad+\frac{1}{2 \pi i} \int\left(\frac{-z_{\alpha \alpha}(\alpha, t)}{(z(\alpha, t)-z(\beta, t))^{2}}+\frac{2 z_{\alpha}^{2}(\alpha, t)}{(z(\alpha, t)-z(\beta, t))^{3}}\right)(1-\rho(\beta)) d \beta .
\end{aligned}
$$

Let

$$
u=z_{\alpha \alpha} .
$$

We take the difference quotient with respect to $\alpha$ on both sides of (5.14) and then multiply both sides by $\eta(\alpha)$. We get

$$
\begin{aligned}
& \eta(\alpha) \bar{u}_{t h}(\alpha, t) \\
&= \frac{\eta(\alpha)}{2 \pi i} \int \frac{-u_{h}(\alpha, t)+u_{h}(\beta, t)}{(z(\alpha+h, t)-z(\beta+h, t))^{2}} \rho(\beta) d \beta \\
&+\frac{1}{2 \pi i h} \int\left(\frac{-u(\alpha, t)+u(\beta, t)}{(z(\alpha+h, t)-z(\beta+h, t))^{2}}-\frac{-u(\alpha, t)+u(\beta, t)}{(z(\alpha, t)-z(\beta, t))^{2}}\right)(\eta(\alpha)-\eta(\beta)) \rho(\beta) d \beta \\
&+\frac{1}{2 \pi i h} \int\left(\frac{-u(\alpha, t)+u(\beta, t)}{(z(\alpha+h, t)-z(\beta+h, t))^{2}}-\frac{-u(\alpha, t)+u(\beta, t)}{(z(\alpha, t)-z(\beta, t))^{2}}\right) \eta(\beta) d \beta \\
&+\frac{1}{\pi i} \int \frac{\left(z_{\alpha}(\alpha+h, t)+z_{\alpha}(\alpha, t)-z_{\beta}(\beta+h, t)-z_{\beta}(\beta, t)\right)\left(\eta(\alpha) z_{\alpha h}(\alpha, t)-\eta(\beta) z_{\beta h}(\beta, t)\right)}{(z(\alpha+h, t)-z(\beta+h, t))^{3}} \rho(\beta) d \beta \\
&+\frac{1}{\pi i} \int \frac{\left(z_{\alpha}(\alpha+h, t)+z_{\alpha}(\alpha, t)-z_{\beta}(\beta+h, t)-z_{\beta}(\beta, t)\right)(\eta(\beta)-\eta(\alpha)) z_{\beta h}(\beta, t)}{(z(\alpha+h, t)-z(\beta+h, t))^{3}} \rho(\beta) d \beta
\end{aligned}
$$




$$
\begin{aligned}
& +\frac{1}{\pi i h} \int\left(\frac{\left(z_{\alpha}(\alpha, t)-z_{\beta}(\beta, t)\right)^{2}}{(z(\alpha+h, t)-z(\beta+h, t))^{3}}-\frac{\left(z_{\alpha}(\alpha, t)-z_{\beta}(\beta, t)\right)^{2}}{(z(\alpha, t)-z(\beta, t))^{3}}\right)(\eta(\alpha)-\eta(\beta)) \rho(\beta) d \beta \\
& +\frac{1}{\pi i h} \int\left(\frac{\left(z_{\alpha}(\alpha, t)-z_{\beta}(\beta, t)\right)^{2}}{(z(\alpha+h, t)-z(\beta+h, t))^{3}}-\frac{\left(z_{\alpha}(\alpha, t)-z_{\beta}(\beta, t)\right)^{2}}{(z(\alpha, t)-z(\beta, t))^{3}}\right) \eta(\beta) d \beta \\
& +\frac{\eta(\alpha)}{2 \pi i} \int \frac{-u(\alpha+h, t)+u(\beta+h, t)}{(z(\alpha+h, t)-z(\beta+h, t))^{2}} \rho_{h}(\beta) d \beta+\frac{\eta(\alpha)}{\pi i} \int \frac{\left(z_{\alpha}(\alpha+h, t)-z_{\beta}(\beta+h, t)\right)^{2}}{(z(\alpha+h, t)-z(\beta+h, t))^{3}} \rho_{h}(\beta) d \beta \\
& +\eta(\alpha) f_{1 h}(\alpha, t) .
\end{aligned}
$$

Let

$$
\begin{aligned}
f_{2}= & \frac{\eta(\alpha)}{2 \pi i} \int \frac{-u(\alpha+h, t)+u(\beta+h, t)}{(z(\alpha+h, t)-z(\beta+h, t))^{2}} \rho_{h}(\beta) d \beta \\
& +\frac{\eta(\alpha)}{\pi i} \int \frac{\left(z_{\alpha}(\alpha+h, t)-z_{\beta}(\beta+h, t)\right)^{2}}{(z(\alpha+h, t)-z(\beta+h, t))^{3}} \rho_{h}(\beta) d \beta, \\
\mathrm{I}= & \frac{1}{2 \pi i h} \int\left(\frac{-u(\alpha, t)+u(\beta, t)}{(z(\alpha+h, t)-z(\beta+h, t))^{2}}-\frac{-u(\alpha, t)+u(\beta, t)}{(z(\alpha, t)-z(\beta, t))^{2}}\right)(\eta(\alpha)-\eta(\beta)) \rho(\beta) d \beta, \\
\mathrm{II}= & \frac{1}{h} \int\left(\frac{-u(\alpha, t)+u(\beta, t)}{(z(\alpha+h, t)-z(\beta+h, t))^{2}}-\frac{-u(\alpha, t)+u(\beta, t)}{(z(\alpha, t)-z(\beta, t))^{2}}\right) \eta(\beta) d \beta, \\
\mathrm{III}= & \frac{1}{\pi i} \int \frac{\left(z_{\alpha}(\alpha+h, t)+z_{\alpha}(\alpha, t)-z_{\beta}(\beta+h, t)-z_{\beta}(\beta, t)\right)\left(\eta(\alpha) z_{\alpha h}(\alpha, t)-\eta(\beta) z_{\beta h}(\beta, t)\right)}{(z(\alpha+h, t)-z(\beta+h, t))^{3}} \rho(\beta) d \beta, \\
\mathrm{IV}= & \frac{1}{\pi i} \int \frac{\left(z_{\alpha}(\alpha+h, t)+z_{\alpha}(\alpha, t)-z_{\beta}(\beta+h, t)-z_{\beta}(\beta, t)\right)(\eta(\beta)-\eta(\alpha)) z_{\beta h}(\beta, t)}{(z+h, t)-z(\beta+h, t))^{3}} \rho(\beta) d \beta, \\
\mathrm{V}= & \frac{1}{\pi i h} \int\left(\frac{\left(z_{\alpha}(\alpha, t)-z_{\beta}(\beta, t)\right)^{2}}{(z(\alpha+h, t)-z(\beta+h, t))^{3}}-\frac{\left(z_{\alpha}(\alpha, t)-z \beta(\beta, t)\right)^{2}}{(z(\alpha, t)-z(\beta, t))^{3}}\right)(\eta(\alpha)-\eta(\beta)) \rho(\beta) d \beta, \\
\mathrm{VI}= & \frac{1}{h} \int\left(\frac{\left(z_{\alpha}(\alpha, t)-z_{\beta}(\beta, t)\right)^{2}}{(z(\alpha+h, t)-z(\beta+h, t))^{3}}-\frac{\left(z_{\alpha}(\alpha, t)-z_{\beta}(\beta, t)\right)^{2}}{\left.(z(\alpha, t)-z(\beta, t))^{3}\right) \eta(\beta) d \beta .}\right.
\end{aligned}
$$

Therefore

$$
\begin{aligned}
& \eta(\alpha) \bar{u}_{t h}(\alpha, t) \\
& =\frac{\eta(\alpha)}{2 \pi i} \int \frac{-u_{h}(\alpha, t)+u_{h}(\beta, t)}{(z(\alpha+h, t)-z(\beta+h, t))^{2}} \rho(\beta) d \beta \\
& \quad+\chi_{Q}(\alpha)\left(\mathrm{I}+\frac{1}{2 \pi i} \mathrm{II}+\mathrm{III}+\mathrm{IV}+\mathrm{V}+\frac{1}{\pi i} \mathrm{VI}+f_{2}+\eta f_{1 h}\right) .
\end{aligned}
$$

We may multiply $\chi_{Q}$ in (5.17) because supp $\eta \subset Q$. For fixed $t \in[0, T]$, we want to estimate $\int_{Q}\left|I+\frac{1}{2 \pi i} \mathrm{II}+\mathrm{III}+\mathrm{IV}+\mathrm{V}+\frac{1}{\pi i} \mathrm{VI}+f_{2}+\eta f_{1 h}\right|^{2} d \alpha$. We rewrite I and $\mathrm{V}$ as

$$
\mathrm{I}=\frac{1}{\pi i h} \int_{0}^{h} \int \frac{(u(\alpha, t)-u(\beta, t))(\eta(\alpha)-\eta(\beta))\left(z_{\alpha}(\alpha+r)-z_{\beta}(\beta+r, t)\right)}{(z(\alpha+r, t)-z(\beta+r, t))^{3}} \rho(\beta) d \beta d r
$$

and

$$
\mathrm{V}=-\frac{3}{\pi i h} \int_{0}^{h} \int \frac{\left(z_{\alpha}(\alpha, t)-z_{\beta}(\beta, t)\right)^{2}(\eta(\alpha)-\eta(\beta))\left(z_{\alpha}(\alpha+r, t)-z_{\beta}(\beta+r, t)\right)}{(z(\alpha+r, t)-z(\beta+r, t))^{4}} \rho(\beta) d \beta d r
$$

We also need to work on II and VI by rewriting them into expressions that we can estimate using existing results such as Lemmas 5.5 and 5.6. We plan to use 
integration by parts. We have

$$
\begin{aligned}
\mathrm{II}= & \frac{1}{h} \int\left(\frac{(-u(\alpha, t)+u(\beta, t)) z_{\beta}(\beta+h, t)}{(z(\alpha+h, t)-z(\beta+h, t))^{2}}-\frac{(-u(\alpha, t)+u(\beta, t)) z_{\beta}(\beta, t)}{(z(\alpha, t)-z(\beta, t))^{2}}\right) \frac{\eta(\beta)}{z_{\beta}(\beta, t)} d \beta \\
& +\frac{1}{h} \int \frac{-u(\alpha, t)+u(\beta, t)}{(z(\alpha+h, t)-z(\beta+h, t))^{2}}\left(1-\frac{z_{\beta}(\beta+h, t)}{z_{\beta}(\beta, t)}\right) \eta(\beta) d \beta=\mathrm{II}_{1}+\mathrm{II}_{2} .
\end{aligned}
$$

Using integration by parts on the first term $\mathrm{II}_{1}$, we rewrite it as

$$
\begin{aligned}
\mathrm{II}_{1}= & \frac{1}{h} \int\left(\frac{u(\alpha, t)-u(\beta, t)}{z(\alpha+h, t)-z(\beta+h, t)}-\frac{u(\alpha, t)-u(\beta, t)}{z(\alpha, t)-z(\beta, t)}\right) \partial_{\beta}\left(\frac{\eta(\beta)}{z_{\beta}(\beta, t)}\right) d \beta \\
& -\frac{1}{h} \int\left(\frac{1}{z(\alpha+h, t)-z(\beta+h, t)}-\frac{1}{z(\alpha, t)-z(\beta, t)}\right) u_{\beta}(\beta, t) \frac{\eta(\beta)}{z_{\beta}(\beta, t)} d \beta \\
= & \frac{1}{h} \int\left(\frac{u(\alpha, t)-u(\beta, t)}{z(\alpha+h, t)-z(\beta+h, t)}-\frac{u(\alpha, t)-u(\beta, t)}{z(\alpha, t)-z(\beta, t)}\right)\left(\frac{\eta^{\prime}(\beta)}{z_{\beta}(\beta, t)}-z_{\beta h}(\beta, t) \frac{\eta(\beta)}{z_{\beta}^{2}(\beta, t)}\right) d \beta \\
& -\int\left(\frac{u(\alpha, t)-u(\beta, t)}{z(\alpha+h, t)-z(\beta+h, t)}-\frac{u(\alpha, t)-u(\beta, t)}{z(\alpha, t)-z(\beta, t)}\right) \frac{\left(z_{\beta \beta}-z_{\beta h}\right)(\beta, t)}{h} \frac{\eta(\beta)}{z_{\beta}^{2}(\beta, t)} d \beta \\
& -\int\left(\frac{1}{z(\alpha+h, t)-z(\beta+h, t)}-\frac{1}{z(\alpha, t)-z(\beta, t)}\right) \frac{\left(u_{\beta}-u_{h}\right)(\beta, t)}{h} \frac{\eta(\beta)}{z_{\beta}(\beta, t)} d \beta \\
& -\frac{1}{h} \int\left(\frac{1}{z(\alpha+h, t)-z(\beta+h, t)}-\frac{1}{z(\alpha, t)-z(\beta, t)}\right) u_{h}(\beta, t) \frac{\eta(\beta)}{z_{\beta}(\beta, t)} d \beta \\
= & \mathrm{II}_{11}+\mathrm{II}_{12}+\mathrm{II}_{13}+\mathrm{II}_{14} .
\end{aligned}
$$

We further rewrite the terms $\mathrm{II}_{11}, \mathrm{II}_{13}$, and $\mathrm{II}_{14}$ :

$$
\begin{aligned}
\mathrm{II}_{11}= & \frac{1}{h} \int\left(\frac{u(\alpha, t)-u(\beta, t)}{z(\alpha+h, t)-z(\beta+h, t)}-\frac{u(\alpha, t)-u(\beta, t)}{z(\alpha, t)-z(\beta, t)}\right)\left(\frac{\eta^{\prime}(\beta)}{z_{\beta}(\beta, t)}-z_{\beta h}(\beta, t) \frac{\eta(\beta)}{z_{\beta}^{2}(\beta, t)}\right) d \beta \\
= & -\frac{1}{h} \int_{0}^{h} \int \frac{\left(z_{\alpha}(\alpha+r, t)-z_{\beta}(\beta+r, t)\right)(u(\alpha, t)-u(\beta, t))}{(z(\alpha+r, t)-z(\beta+r, t))^{2}} \\
& \cdot\left(\frac{\eta^{\prime}(\beta)}{z_{\beta}(\beta, t)}-z_{\beta h}(\beta, t) \frac{\eta(\beta)}{z_{\beta}^{2}(\beta, t)}\right) d \beta d r, \\
\mathrm{II}_{13}= & -\int\left(\frac{1}{z(\alpha+h, t)-z(\beta+h, t)}-\frac{1}{z(\alpha, t)-z(\beta, t)}\right) \frac{\left(u_{\beta}-u_{h}\right)(\beta, t)}{h} \frac{\eta(\beta)}{z_{\beta}(\beta, t)} d \beta \\
= & \left.\int \frac{(z(\alpha+h, t)-z(\alpha, t)-z(\beta+h, t)+z(\beta, t))}{(z(\alpha+h, t)-z(\beta+h, t))(z(\alpha, t)-z(\beta, t))} \frac{1}{h}-u_{h}\right)(\beta, t) \\
\mathrm{I}_{14}(\beta) & -\frac{1}{h} \int\left(\frac{1}{z(\alpha+h, t)} d \beta,\right. \\
\mathrm{I}_{14}(\beta+h, t) & \left.-\frac{1}{z(\alpha, t)-z(\beta, t)}\right) u_{h}(\beta, t) \frac{\eta(\beta)}{z_{\beta}(\beta, t)} d \beta \\
= & \frac{1}{h} \int_{0}^{h} \int \frac{z_{\alpha}(\alpha+r, t)-z_{\beta}(\beta+r, t)}{(z(\alpha+r, t)-z(\beta+r, t))^{2}} u_{h}(\beta, t) \frac{\eta(\beta)}{z_{\beta}(\beta, t)} d \beta d r .
\end{aligned}
$$

Similarly, for VI we have

$$
\begin{aligned}
\mathrm{VI}= & \frac{1}{h} \int\left(\frac{\left(z_{\alpha}(\alpha, t)-z_{\beta}(\beta, t)\right)^{2} z_{\beta}(\beta+h, t)}{(z(\alpha+h, t)-z(\beta+h, t))^{3}}-\frac{\left(z_{\alpha}(\alpha, t)-z_{\beta}(\beta, t)\right)^{2} z_{\beta}(\beta, t)}{(z(\alpha, t)-z(\beta, t))^{3}}\right) \frac{\eta(\beta)}{z_{\beta}(\beta, t)} d \beta \\
& -\int\left(\frac{\left(z_{\alpha}(\alpha, t)-z_{\beta}(\beta, t)\right)^{2}}{(z(\alpha+h, t)-z(\beta+h, t))^{3}}\right) z_{\beta h}(\beta, t) \frac{\eta(\beta)}{z_{\beta}(\beta, t)} d \beta=\mathrm{VI}_{1}+\mathrm{VI}_{2} .
\end{aligned}
$$


Using integration by parts on the first term, $\mathrm{VI}_{1}$, we get

$$
\begin{aligned}
\mathrm{VI}_{1}= & \int\left(\frac{z_{\alpha}(\alpha, t)-z_{\beta}(\beta, t)}{(z(\alpha+h, t)-z(\beta+h, t))^{2}}-\frac{z_{\alpha}(\alpha, t)-z_{\beta}(\beta, t)}{(z(\alpha, t)-z(\beta, t))^{2}}\right) \frac{\left(z_{\beta \beta}-z_{\beta h}\right)(\beta, t)}{h} \frac{\eta(\beta)}{z_{\beta}(\beta, t)} d \beta \\
& +\frac{1}{h} \int\left(\frac{z_{\alpha}(\alpha, t)-z_{\beta}(\beta, t)}{(z(\alpha+h, t)-z(\beta+h, t))^{2}}-\frac{z_{\alpha}(\alpha, t)-z_{\beta}(\beta, t)}{(z(\alpha, t)-z(\beta, t))^{2}}\right) z_{\beta h}(\beta, t) \frac{\eta(\beta)}{z_{\beta}(\beta, t)} d \beta \\
& -\frac{1}{2 h} \int\left(\frac{\left(z_{\alpha}(\alpha, t)-z_{\beta}(\beta, t)\right)^{2}}{(z(\alpha+h, t)-z(\beta+h, t))^{2}}-\frac{\left(z_{\alpha}(\alpha, t)-z_{\beta}(\beta, t)\right)^{2}}{(z(\alpha, t)-z(\beta, t))^{2}}\right) \\
& \cdot\left(\frac{\eta^{\prime}(\beta)}{z_{\beta}(\beta, t)}-z_{\beta h}(\beta, t) \frac{\eta(\beta)}{z_{\beta}^{2}(\beta, t)}\right) d \beta \\
+ & \frac{1}{2} \int\left(\frac{\left(z_{\alpha}(\alpha, t)-z_{\beta}(\beta, t)\right)^{2}}{(z(\alpha+h, t)-z(\beta+h, t))^{2}}-\frac{\left(z_{\alpha}(\alpha, t)-z_{\beta}(\beta, t)\right)^{2}}{(z(\alpha, t)-z(\beta, t))^{2}}\right) \\
& \cdot \frac{\left(z_{\beta \beta}-z_{\beta h}\right)(\beta, t)}{h} \frac{\eta(\beta)}{z_{\beta}^{2}(\beta, t)} d \beta \\
= & \mathrm{VI}_{11}+\mathrm{VI}_{12}+\mathrm{VI}_{13}+\mathrm{VI}_{14} .
\end{aligned}
$$

We further rewrite $\mathrm{VI}_{12}$ and $\mathrm{VI}_{13}$ as

$$
\begin{gathered}
\mathrm{VI}_{12}=\frac{1}{h} \int\left(\frac{z_{\alpha}(\alpha, t)-z_{\beta}(\beta, t)}{(z(\alpha+h, t)-z(\beta+h, t))^{2}}-\frac{z_{\alpha}(\alpha, t)-z_{\beta}(\beta, t)}{(z(\alpha, t)-z(\beta, t))^{2}}\right) z_{\beta h}(\beta, t) \frac{\eta(\beta)}{z_{\beta}(\beta, t)} d \beta \\
=-\frac{2}{h} \int_{0}^{h} \int \frac{\left(z_{\alpha}(\alpha, t)-z_{\beta}(\beta, t)\right)\left(z_{\alpha}(\alpha+r, t)-z_{\beta}(\beta+r, t)\right)}{(z(\alpha+r, t)-z(\beta+r, t))^{3}} \\
\cdot z_{\beta h}(\beta, t) \frac{\eta(\beta)}{z_{\beta}(\beta, t)} d \beta d r
\end{gathered}
$$

and

$$
\begin{aligned}
& \mathrm{VI}_{13}=-\frac{1}{2 h} \int\left(\frac{\left(z_{\alpha}(\alpha, t)-z_{\beta}(\beta, t)\right)^{2}}{(z(\alpha+h, t)-z(\beta+h, t))^{2}}-\frac{\left(z_{\alpha}(\alpha, t)-z_{\beta}(\beta, t)\right)^{2}}{(z(\alpha, t)-z(\beta, t))^{2}}\right) \\
& \cdot\left(\frac{\eta^{\prime}(\beta)}{z_{\beta}(\beta, t)}-z_{\beta h}(\beta, t) \frac{\eta(\beta)}{z_{\beta}^{2}(\beta, t)}\right) d \beta \\
&=\frac{1}{h} \int_{0}^{h} \int \frac{\left(z_{\alpha}(\alpha, t)-z_{\beta}(\beta, t)\right)^{2}\left(z_{\alpha}(\alpha+r, t)-z_{\beta}(\beta+r, t)\right)}{(z(\alpha+r, t)-z(\beta+r, t))^{3}} \\
& \cdot\left(\frac{\eta^{\prime}(\beta)}{z_{\beta}(\beta, t)}-z_{\beta h}(\beta, t) \frac{\eta(\beta)}{z_{\beta}^{2}(\beta, t)}\right) d \beta d r .
\end{aligned}
$$

We can now estimate the $L^{2}(Q)$ norm of terms I through VI using their new expressions. We apply Lemma 5.6 to I, V, III, IV, $\mathrm{II}_{2}, \mathrm{II}_{11}, \mathrm{VI}_{2}, \mathrm{VI}_{12}$, and $\mathrm{VI}_{13}$. For $\mathrm{II}_{12}, \mathrm{II}_{14}, \mathrm{VI}_{11}$, and $\mathrm{VI}_{14}$ we apply Lemmas 5.4 and 5.5. For $\mathrm{II}_{13}$ and fixed $t \in[0, T]$, we first replace the curve $z=z(\alpha, t)$ by its reflective periodic extension (extend $z_{\alpha}(\alpha, t)$ in $\alpha$ ) from $\alpha \in 2 Q$ to $\mathbb{R}$ and then apply the Tb theorem. Notice that the new curve is a chord-arc when $|Q|$ is small enough, and the reflective periodic extension doesn't change $\mathrm{II}_{13}$ for $\alpha \in Q$. 
Let

$$
\varpi\left(z_{\alpha}, h\right)=\sup _{\substack{\alpha, \beta \in \mathcal{Q} \\ t \in \mathcal{T} \\|\alpha-\beta| \leq|h|}}\left|z_{\beta}(\beta, t)-z_{\alpha}(\alpha, t)\right|
$$

Notice that

$$
\begin{aligned}
\left\|z_{\alpha \alpha} z_{\alpha h} \eta\right\|_{L^{2}(\mathcal{I})} & \leq\left\|z_{\alpha \alpha}\right\|_{L^{2}(\mathcal{I})}\left\|z_{\alpha h} \eta\right\|_{L^{\infty}(\mathcal{I})} \\
& \leq|\mathcal{I}|^{1 / 2}\left\|z_{\alpha \alpha}\right\|_{L^{2}(\mathcal{I})}\left\|\partial_{\alpha}\left(z_{\alpha h} \eta\right)\right\|_{L^{2}(\mathcal{I})}
\end{aligned}
$$

for any interval $\mathcal{I} \supset \operatorname{supp} \eta$. We get, for $0<|h| \leq \frac{1}{8}|Q|$, that there exist constants $c_{0}$ (depending on $m$ and $M$ ) and $c_{1}$ (depending on $m, M$, and $|Q|$ ) such that for $t \in[0, T]$,

$$
\begin{aligned}
& \left(\int_{Q}\left|I+\frac{1}{2 \pi i} \mathrm{II}+\mathrm{III}+\mathrm{IV}+\mathrm{V}+\frac{1}{\pi i} \mathrm{VI}\right|^{2} d \alpha\right)^{1 / 2} \\
& \leq c_{1}\left(1+\left\|z_{\alpha \alpha}(\cdot, t)\right\|_{L^{2}(3 Q)}\right)^{4}\left\|\partial_{\alpha}^{2} z_{\alpha}(\cdot, t)\right\|_{L^{2}(3 Q)}\left\|\eta u_{h}(\cdot, t)\right\|_{L^{2}(Q)} \\
& \quad+c_{0}|Q|^{1 / 2}\left\|\partial_{\alpha}^{2} z_{\alpha}(\cdot, t)\right\|_{L^{2}(3 Q)}\left\|\eta \tilde{u}_{h}(\cdot, t)\right\|_{L^{2}(3 Q)} \\
& \quad+c_{0} \varpi\left(z_{\alpha}, h\right)\left\|\eta \tilde{u}_{\alpha h}(\cdot, t)\right\|_{L^{2}(3 Q)} \\
& \quad+c_{1}\left(1+\left\|z_{\alpha \alpha}(\cdot, t)\right\|_{L^{2}(3 Q)}\right)^{5}\left\|z_{\alpha}(\cdot, t)\right\|_{H^{2}(3 Q)} .
\end{aligned}
$$

For $f_{2}$ and $\eta f_{1 h}$, we notice that for $0<|h| \leq \frac{1}{8}|Q|, \beta \in \frac{11}{8} Q, \rho_{h}(\beta)=0$, and $\rho^{\prime}(\beta)=0$ for $\beta \in \frac{3}{2} Q$.

Recall supp $\eta \subset Q$. We directly estimate the integrals in the expressions of $f_{2}$ and $\partial_{\alpha} f_{1}$ and use

$$
f_{1 h}(\alpha, t)=\frac{1}{h} \int_{0}^{h} \partial_{\alpha} f_{1}(\alpha+r, t) d r
$$

to estimate $f_{1 h}$ through $\partial_{\alpha} f_{1}$. We get

$$
\left(\int_{Q}\left|f_{2}(\alpha, t)+\eta(\alpha) f_{1 h}(\alpha, t)\right|^{2} d \alpha\right)^{1 / 2} \leq c_{2}\left\|z_{\alpha \alpha}(\cdot, t)\right\|_{H^{1}(3 Q)}+c_{2}
$$

where $c_{2}$ depends on $m, M$, and $|Q|$ only.

We now consider the term

$$
\frac{\eta(\alpha)}{2 \pi i} \int \frac{-u_{h}(\alpha, t)+u_{h}(\beta, t)}{(z(\alpha+h, t)-z(\beta+h, t))^{2}} \rho(\beta) d \beta
$$

in (5.17). We note that if we replace in (5.22) the curve $z(\alpha, t)$ by its reflective periodic extension (extend $z_{\alpha}(\alpha, t)$ in $\alpha$ ) from $\alpha \in 3 Q$ to $\mathbb{R}$, the quantity in (5.22) remains unchanged. For $|Q|$ small enough (independent of $t \in[0, T]$ ), the new curve is still chord-arc. From here on we replace the curve $z=z(\alpha, t)$ in (5.22) 
by its reflective periodical extension, and we use the same notation $z=z(\alpha, t)$ to indicate the new curve.

We rewrite the quantity

$$
\begin{aligned}
\eta(\alpha) & \int \frac{-u_{h}(\alpha, t)+u_{h}(\beta, t)}{(z(\alpha+h, t)-z(\beta+h, t))^{2}} \rho(\beta) d \beta \\
= & \int \frac{\left(-\eta(\alpha) u_{h}(\alpha, t)+\eta(\beta) u_{h}(\beta, h)\right) \rho(\beta)}{(z(\alpha+h, t)-z(\beta+h, t))^{2}} d \beta \\
& +\int \frac{(\eta(\alpha)-\eta(\beta)) u_{h}(\beta, h) \rho(\beta)}{(z(\alpha+h, t)-z(\beta+h, t))^{2}} d \beta .
\end{aligned}
$$

Let

$$
A=\int \frac{\left(-\eta(\alpha) u_{h}(\alpha, t)+\eta(\beta) u_{h}(\beta, h)\right) \rho(\beta)}{(z(\alpha+h, t)-z(\beta+h, t))^{2}} d \beta
$$

and

$$
B=\int \frac{(\eta(\alpha)-\eta(\beta)) u_{h}(\beta, h) \rho(\beta)}{(z(\alpha+h, t)-z(\beta+h, t))^{2}} d \beta .
$$

We further rewrite $A$ :

$$
\begin{aligned}
A= & -\eta(\alpha) u_{h}(\alpha, t) \int \frac{z_{\beta}(\beta+h, t)}{(z(\alpha+h, t)-z(\beta+h, t))^{2}}\left(\frac{\rho(\beta)}{z_{\beta}(\beta+h, t)}-\frac{\rho(\alpha)}{z_{\alpha}(\alpha+h, t)}\right) d \beta \\
& +\frac{1}{z_{\alpha}(\alpha+h, t)} \int\left(\frac{z_{\alpha}(\alpha+h, t) z_{\beta}(\beta+h, t)}{(z(\alpha+h, t)-z(\beta+h, t))^{2}}-\frac{1}{(\alpha-\beta)^{2}}\right) \frac{\eta(\beta) u_{h}(\beta, t)}{z_{\beta}(\beta+h, t)} d \beta \\
& +\frac{1}{z_{\alpha}(\alpha+h, t)} \int \frac{1}{(\alpha-\beta)^{2}}\left(\frac{\eta(\beta) u_{h}(\beta, t)}{z_{\beta}(\beta+h, t)}-\frac{\eta(\alpha) u_{h}(\alpha, t)}{z_{\alpha}(\alpha+h, t)}\right) d \beta
\end{aligned}
$$

Let

$$
\begin{aligned}
F(\alpha, t) & =\int \frac{z_{\beta}(\beta+h, t)}{(z(\alpha+h, t)-z(\beta+h, t))^{2}}\left(\frac{\rho(\beta)}{z_{\beta}(\beta+h, t)}-\frac{\rho(\alpha)}{z_{\alpha}(\alpha+h, t)}\right) d \beta \\
& =-\int \frac{1}{z(\alpha+h, t)-z(\beta+h, t)} \partial_{\beta}\left(\frac{\rho(\beta)}{z_{\beta}(\beta+h, t)}\right) d \beta .
\end{aligned}
$$

Notice that

$$
\begin{aligned}
\partial_{\alpha} F(\alpha, t)= & \int \frac{z_{\alpha}(\alpha+h, t)-z_{\beta}(\beta+h, t)}{(z(\alpha+h, t)-z(\beta+h, t))^{2}} \partial_{\beta}\left(\frac{\rho(\beta)}{z_{\beta}(\beta+h, t)}\right) d \beta \\
& -\int \frac{1}{z(\alpha+h, t)-z(\beta+h, t)} \partial_{\beta}^{2}\left(\frac{\rho(\beta)}{z_{\beta}(\beta+h, t)}\right) d \beta .
\end{aligned}
$$

Let

$$
A_{2}=\frac{1}{z_{\alpha}(\alpha+h, t)} \int\left(\frac{z_{\alpha}(\alpha+h, t) z_{\beta}(\beta+h, t)}{(z(\alpha+h, t)-z(\beta+h, t))^{2}}-\frac{1}{(\alpha-\beta)^{2}}\right) \frac{\eta(\beta) u_{h}(\beta, t)}{z_{\beta}(\beta+h, t)} d \beta .
$$


Therefore

$$
\begin{aligned}
& \frac{\eta(\alpha)}{2 \pi i} \int \frac{-u_{h}(\alpha, t)+u_{h}(\beta, t)}{(z(\alpha+h, t)-z(\beta+h, t))^{2}} \rho(\beta) d \beta \\
& \quad=\frac{1}{2 \pi i z_{\alpha}(\alpha+h, t)} \int \frac{1}{(\alpha-\beta)^{2}}\left(\frac{\eta(\beta) u_{h}(\beta, t)}{z_{\beta}(\beta+h, t)}-\frac{\eta(\alpha) u_{h}(\alpha, t)}{z_{\alpha}(\alpha+h, t)}\right) d \beta \\
& \quad-\frac{1}{2 \pi i} \eta(\alpha) u_{h}(\alpha, t) F(\alpha, t)+\frac{1}{2 \pi i} A_{2}+\frac{1}{2 \pi i} B .
\end{aligned}
$$

We apply Lemma 5.5 to $B$ and $F(\alpha, t)$, the T1 theorem (see David and Journe [18]) to $A_{2}$, Lemma 5.6 to $\partial_{\alpha} F(\alpha, t)$, and Lemma 5.4; we get

$$
\begin{aligned}
& \left(\int\left|-\frac{1}{2 \pi i} \eta(\alpha) u_{h}(\alpha, t) F(\alpha, t)+\frac{1}{2 \pi i} A_{2}+\frac{1}{2 \pi i} B\right|^{2} d \alpha\right)^{1 / 2} \\
& \leq c_{3}\left\|\eta u_{h}(\cdot, t)\right\|_{L^{2}(3 Q)}\|F(\cdot, t)\|_{L^{\infty}(3 Q)} \\
& \quad+c_{3}\left\|z_{\alpha \alpha}(\cdot, t)\right\|_{L^{\infty}(3 Q)}\left\|\eta u_{h}(\cdot, t)\right\|_{L^{2}(3 Q)}+c_{3}\left\|\rho u_{h}(\cdot, t)\right\|_{L^{2}(3 Q)} \\
& \leq c_{4}\left(1+\left\|z_{\alpha \alpha}(\cdot, t)\right\|_{L^{2}(3 Q)}\right)^{2}\left\|\partial_{\alpha}^{2} z_{\alpha}(\cdot, t)\right\|_{L^{2}(3 Q)}\left\|\eta u_{h}(\cdot, t)\right\|_{L^{2}(3 Q)} \\
& \quad+c_{4}\left(1+\left\|z_{\alpha \alpha}(\cdot, t)\right\|_{L^{2}(3 Q)}\right)^{3}\left\|u_{\alpha}(\cdot, t)\right\|_{L^{2}(3 Q)}
\end{aligned}
$$

where $c_{3}$ and $c_{4}$ depend on $m, M$, and $|Q|$.

Summing up the above calculation, we have

(5.25) $\eta(\alpha) \bar{u}_{t h}$

$$
=\frac{1}{2 \pi i z_{\alpha}(\alpha+h, t)} \int \frac{1}{(\alpha-\beta)^{2}}\left(\frac{\eta(\beta) u_{h}(\beta, t)}{z_{\beta}(\beta+h, t)}-\frac{\eta(\alpha) u_{h}(\alpha, t)}{z_{\alpha}(\alpha+h, t)}\right) d \beta+G(\alpha, t)
$$

where

$$
\begin{aligned}
G(\alpha, t)= & -\frac{1}{2 \pi i} \eta(\alpha) u_{h}(\alpha, t) F(\alpha, t)+\frac{1}{2 \pi i} A_{2}+\frac{1}{2 \pi i} B \\
& +\chi_{Q}(\alpha)\left(\mathrm{I}+\frac{1}{2 \pi i} \mathrm{II}+\mathrm{III}+\mathrm{IV}+\mathrm{V}+\frac{1}{\pi i} \mathrm{VI}+f_{2}+\eta f_{1 h}\right)
\end{aligned}
$$

and

$$
\begin{aligned}
& \left(\int|G(\alpha, t)|^{2} d \alpha\right)^{1 / 2} \\
& \quad \leq c_{0}^{\prime}\left(1+\left\|z_{\alpha \alpha}(\cdot, t)\right\|_{L^{2}(3 Q)}\right)^{4}\left\|\partial_{\alpha}^{2} z_{\alpha}(\cdot, t)\right\|_{L^{2}(3 Q)}\left\|\eta u_{h}(\cdot, t)\right\|_{L^{2}(Q)} \\
& \quad+c_{0}|Q|^{1 / 2}\left\|\partial_{\alpha}^{2} z_{\alpha}(\cdot, t)\right\|_{L^{2}(3 Q)}\left\|\eta \tilde{u}_{h}(\cdot, t)\right\|_{L^{2}(3 Q)} \\
& \quad+c_{0} \varpi\left(z_{\alpha}, h\right)\left\|\eta \tilde{u}_{\alpha h}(\cdot, t)\right\|_{L^{2}(3 Q)} \\
& \quad+c_{1}^{\prime}\left(1+\left\|z_{\alpha \alpha}(\cdot, t)\right\|_{L^{2}(3 Q)}\right)^{5}\left\|z_{\alpha}(\cdot, t)\right\|_{H^{2}(3 Q)}+c_{2}\left\|z_{\alpha \alpha}(\cdot, t)\right\|_{H^{1}(3 Q)} \\
& \quad+c_{2}
\end{aligned}
$$


where $c_{0}$ depends on $m$ and $M$ only, and $c_{0}^{\prime}, c_{1}^{\prime}$, and $c_{2}$ depend on $m, M$, and $|Q|$.

We now consider

$$
\begin{aligned}
\int \mid & \eta(\alpha) \bar{u}_{t h}(\alpha, t) \\
& -\left.\frac{1}{2 \pi i z_{\alpha}(\alpha+h, t)} \int \frac{1}{(\alpha-\beta)^{2}}\left(\frac{\eta(\beta) u_{h}(\beta, t)}{z_{\beta}(\beta+h, t)}-\frac{\eta(\alpha) u_{h}(\alpha, t)}{z_{\alpha}(\alpha+h, t)}\right) d \beta\right|^{2} d \alpha \\
= & \int\left|\eta(\alpha) \bar{u}_{t h}(\alpha, t)+\frac{1}{2 i z_{\alpha}(\alpha+h, t)} H\left(\partial_{\alpha}\left(\frac{\eta(\alpha) u_{h}(\alpha, t)}{z_{\alpha}(\alpha+h, t)}\right)\right)\right|^{2} d \alpha \\
= & \int\left|\eta(\alpha) \bar{u}_{t h}(\alpha, t)\right|^{2} d \alpha+\int\left|\frac{1}{2 z_{\alpha}(\alpha+h, t)} H\left(\partial_{\alpha}\left(\frac{\eta(\alpha) u_{h}(\alpha, t)}{z_{\alpha}(\alpha+h, t)}\right)\right)\right|^{2} d \alpha \\
& +\operatorname{Im} \int \frac{\eta(\alpha) u_{t h}(\alpha, t)}{z_{\alpha}(\alpha+h, t)} H\left(\partial_{\alpha}\left(\frac{\eta(\alpha) u_{h}(\alpha, t)}{z_{\alpha}(\alpha+h, t)}\right)\right) d \alpha
\end{aligned}
$$

where

$$
\begin{aligned}
\int & \frac{\eta(\alpha) u_{t h}(\alpha, t)}{z_{\alpha}(\alpha+h, t)} H\left(\partial_{\alpha}\left(\frac{\eta(\alpha) u_{h}(\alpha, t)}{z_{\alpha}(\alpha+h, t)}\right)\right) d \alpha \\
= & \int \partial_{t}\left(\frac{\eta(\alpha) u_{h}(\alpha, t)}{z_{\alpha}(\alpha+h, t)}\right) H\left(\partial_{\alpha}\left(\frac{\eta(\alpha) u_{h}(\alpha, t)}{z_{\alpha}(\alpha+h, t)}\right)\right) d \alpha \\
& +\int \frac{\eta(\alpha) u_{h}(\alpha, t) z_{t \alpha}(\alpha+h, t)}{z_{\alpha}^{2}(\alpha+h, t)} H\left(\partial_{\alpha}\left(\frac{\eta(\alpha) u_{h}(\alpha, t)}{z_{\alpha}(\alpha+h, t)}\right)\right) d \alpha \\
= & \frac{1}{4 \pi} \frac{d}{d t} \iint \frac{1}{(\alpha-\beta)^{2}}\left(\frac{\eta(\alpha) u_{h}(\alpha, t)}{z_{\alpha}(\alpha+h, t)}-\frac{\eta(\beta) u_{h}(\beta, t)}{z_{\beta}(\beta+h, t)}\right)^{2} d \alpha d \beta \\
& +\int \frac{\eta(\alpha) u_{h}(\alpha, t) z_{t \alpha}(\alpha+h, t)}{z_{\alpha}^{2}(\alpha+h, t)} H\left(\partial_{\alpha}\left(\frac{\eta(\alpha) u_{h}(\alpha, t)}{z_{\alpha}(\alpha+h, t)}\right)\right) d \alpha
\end{aligned}
$$

and

$$
\begin{aligned}
\mid \int & \frac{\eta(\alpha) u_{h}(\alpha, t) z_{t \alpha}(\alpha+h, t)}{z_{\alpha}^{2}(\alpha+h, t)} H\left(\partial_{\alpha}\left(\frac{\eta(\alpha) u_{h}(\alpha, t)}{z_{\alpha}(\alpha+h, t)}\right)\right) d \alpha \mid \\
\leq & \frac{1}{m^{2}}\left\|\eta u_{h}(\cdot, t)\right\|_{L^{2}(3 Q)}\left\|z_{t \alpha}(\cdot, t)\right\|_{L^{\infty}(3 Q)}\left\|\partial_{\alpha}\left(\frac{\eta(\cdot) u_{h}(\cdot, t)}{z_{\alpha}(\cdot+h, t)}\right)\right\|_{L^{2}(3 Q)} \\
\leq & \frac{1}{16 M^{2}}\left\|\partial_{\alpha}\left(\frac{\eta(\cdot) u_{h}(\cdot, t)}{z_{\alpha}(\cdot+h, t)}\right)\right\|_{L^{2}(3 Q)}^{2} \\
& +\frac{4 M^{2}}{m^{4}}\left\|\eta u_{h}(\cdot, t)\right\|_{L^{2}(3 Q)}^{2}\left\|z_{t \alpha}(\cdot, t)\right\|_{H^{1}(3 Q)}^{2} .
\end{aligned}
$$


Therefore from (5.25) and (5.27) we get

$$
\begin{aligned}
\int\left|\eta(\alpha) u_{t h}(\alpha, t)\right|^{2} d \alpha+\frac{3}{16 M^{2}} \int\left|\partial_{\alpha}\left(\frac{\eta(\alpha) u_{h}(\alpha, t)}{z_{\alpha}(\alpha+h, t)}\right)\right|^{2} d \alpha \\
\leq-\frac{1}{4 \pi} \operatorname{Im} \frac{d}{d t} \iint \frac{1}{(\alpha-\beta)^{2}}\left(\frac{\eta(\alpha) u_{h}(\alpha, t)}{z_{\alpha}(\alpha+h, t)}-\frac{\eta(\beta) u_{h}(\beta, t)}{z_{\beta}(\beta+h, t)}\right)^{2} d \alpha d \beta \\
\quad+\frac{4 M^{2}}{m^{4}}\left\|\eta u_{h}(\cdot, t)\right\|_{L^{2}(3 Q)}^{2}\left\|z_{t \alpha}(\cdot, t)\right\|_{H^{1}(3 Q)}^{2}+\int|G(\alpha, t)|^{2} d \alpha .
\end{aligned}
$$

We note that (5.23) with estimate (5.24) holds for any function $u$, and (5.28) holds for any function $u$ satisfying (5.25).

\section{$H^{1}$ Regularity of $\partial_{\alpha}^{2} z_{\alpha}$}

Assume that $\mathcal{T}=\left[T_{1}, T_{2}\right]$. Let $\delta(t)=\left(t-T_{1}\right)^{2}\left(T_{2}-t\right)^{2}$ for $t \in \mathcal{T}$, and $\delta(t)=0$ for $t \notin \mathcal{T}$. From the fact that

$$
\sup _{t \in \mathcal{T}}|g(t)| \leq \frac{1}{|\mathcal{T}|} \int_{\mathcal{T}}|g(t)| d t+\int_{\mathcal{T}}\left|g^{\prime}(t)\right| d t,
$$

we have

$$
\sup _{t \in \mathcal{T}}\left\|z_{\alpha \alpha}(\cdot, t)\right\|_{L^{2}(3 Q)}^{2} \leq \frac{2}{|\mathcal{T}|}\left\|z_{\alpha \alpha}\right\|_{L^{2}(3 Q \times \mathcal{T})}^{2}+|\mathcal{T}|\left\|z_{t \alpha \alpha}\right\|_{L^{2}(3 Q \times \mathcal{T})}^{2},
$$

and for any $\epsilon>0$,

$$
\begin{aligned}
& \sup _{t \in \mathcal{T}} \delta(t)\left\|\eta u_{h}(\cdot, t)\right\|_{L^{2}(3 Q)}^{2} \\
& \quad \leq \epsilon \iint \delta(t)\left|\eta(\alpha) u_{t h}(\alpha, t)\right|^{2} d \alpha d t+\left(3|\mathcal{T}|^{3}+\frac{|\mathcal{T}|^{4}}{\epsilon}\right)\left\|u_{\alpha}\right\|_{L^{2}(3 Q \times \mathcal{T})}^{2} .
\end{aligned}
$$

Since for any function $g$

$$
\eta(\alpha) \tilde{g}_{h}(\alpha)=\frac{1}{h^{2}} \int_{0}^{h} \eta(\alpha)(g(\alpha)-g(\alpha+s)) d s=-\frac{1}{h^{2}} \int_{0}^{h} s \eta(\alpha) g_{s}(\alpha) d s,
$$

we have

$$
\left\|\eta \tilde{u}_{\alpha h}(\cdot, t)\right\|_{L^{2}(3 Q)}^{2} \leq \frac{1}{h^{3}} \int_{0}^{h} s^{2}\left\|\eta u_{\alpha s}(\cdot, t)\right\|_{L^{2}(3 Q)}^{2} d s,
$$

and for any $\epsilon>0$,

$$
\begin{aligned}
\sup _{t \in \mathcal{T}} \delta(t)\left\|\eta \tilde{u}_{h}(\cdot, t)\right\|_{L^{2}(3 Q)}^{2} \leq & \frac{\epsilon}{h^{3}} \int_{0}^{h} s^{2} \iint \delta(t)\left|\eta(\alpha) u_{t s}(\alpha, t)\right|^{2} d \alpha d t d s \\
& +\left(3|\mathcal{T}|^{3}+\frac{|\mathcal{T}|^{4}}{\epsilon}\right)\left\|u_{\alpha}\right\|_{L^{2}(3 Q \times \mathcal{T})}^{2}
\end{aligned}
$$


Furthermore, using the fact that $\left|\delta^{\prime}(t)\right|^{2} \leq 4|\mathcal{T}|^{2} \delta(t)$ for $t \in \mathcal{T}$,

$$
\begin{aligned}
& \left|\frac{-1}{\pi} \int \delta(t) \frac{d}{d t} \iint \frac{1}{(\alpha-\beta)^{2}}\left(\frac{\eta(\alpha) u_{h}(\alpha, t)}{z_{\alpha}(\alpha+h, t)}-\frac{\eta(\beta) u_{h}(\beta, t)}{z_{\beta}(\beta+h, t)}\right)^{2} d \alpha d \beta d t\right| \\
& \quad=\left|\frac{1}{\pi} \int \delta^{\prime}(t) \iint \frac{1}{(\alpha-\beta)^{2}}\left(\frac{\eta(\alpha) u_{h}(\alpha, t)}{z_{\alpha}(\alpha+h, t)}-\frac{\eta(\beta) u_{h}(\beta, t)}{z_{\beta}(\beta+h, t)}\right)^{2} d \alpha d \beta d t\right| \\
& \quad=2\left|\int \delta^{\prime}(t) \int \frac{\eta(\alpha) u_{h}(\alpha, t)}{z_{\alpha}(\alpha+h, t)} H\left(\partial_{\alpha}\left(\frac{\eta(\alpha) u_{h}(\alpha, t)}{z_{\alpha}(\alpha+h, t)}\right)\right) d \alpha d t\right| \\
& \quad \leq \frac{1}{4 M^{2}} \int \delta(t) \int\left|\partial_{\alpha}\left(\frac{\eta(\alpha) u_{h}(\alpha, t)}{z_{\alpha}(\alpha+h, t)}\right)\right|^{2} d \alpha d t+\frac{16 M^{2}|\mathcal{T}|^{2}}{m^{2}}\left\|u_{\alpha}\right\|_{L^{2}(3 Q \times \mathcal{T})}^{2} .
\end{aligned}
$$

We now multiply both sides of (5.28) by $\delta(t)$ and integrate with respect to $t$. Using the estimates from (5.26) through (5.33), we have, for any $\epsilon>0$,

$$
\begin{aligned}
\iint \delta(t)\left|\eta(\alpha) u_{t h}(\alpha, t)\right|^{2} d \alpha d t \\
+\frac{1}{8 M^{2}} \iint \delta(t)\left|\partial_{\alpha}\left(\frac{\eta(\alpha) u_{h}(\alpha, t)}{z_{\alpha}(\alpha+h, t)}\right)\right|^{2} d \alpha d t \\
\leq \epsilon \iint \delta(t)\left|\eta(\alpha) u_{t h}(\alpha, t)\right|^{2} d \alpha d t \\
\quad+\epsilon \frac{1}{h^{3}} \int_{0}^{h} s^{2} \iint \delta(t)\left|\eta(\alpha) u_{t s}(\alpha, t)\right|^{2} d \alpha d t d s \\
\quad+2 c_{0}^{2} \varpi^{2}\left(z_{\alpha}, h\right) \frac{1}{h^{3}} \int_{0}^{h} s^{2} \iint \delta(t)\left|\eta(\alpha) u_{\alpha s}(\alpha, t)\right|^{2} d \alpha d t d s+K_{1}
\end{aligned}
$$

where $K_{1}<\infty$ is a constant depending on $m, M, 1 / \epsilon,|Q|,|\mathcal{T}|,\left\|z_{\alpha}\right\|_{H^{1}(3 Q \times \mathcal{T})}$, and $\left\|z_{\alpha \alpha}\right\|_{H^{1}(3 Q \times \mathcal{T})}$; in particular, $K_{1}$ is independent of $h$. Multiplying (5.34) by $h^{3 / 2}$ and then integrating with respect to $h$ from 0 to $l$, we get

$$
\begin{aligned}
& \frac{1}{l^{5 / 2}} \int_{0}^{l} h^{3 / 2} \iint \delta(t)\left|\eta(\alpha) u_{t h}(\alpha, t)\right|^{2} d \alpha d t d h \\
& +\frac{1}{8 M^{2} l^{5 / 2}} \int_{0}^{l} h^{3 / 2} \iint \delta(t)\left|\partial_{\alpha}\left(\frac{\eta(\alpha) u_{h}(\alpha, t)}{z_{\alpha}(\alpha+h, t)}\right)\right|^{2} d \alpha d t d h \\
& \quad \leq \frac{3 \epsilon}{l^{5 / 2}} \int_{0}^{l} h^{3 / 2} \iint \delta(t)\left|\eta(\alpha) u_{t h}(\alpha, t)\right|^{2} d \alpha d t d h \\
& \quad+4 c_{0}^{2} \varpi^{2}\left(z_{\alpha}, l\right) \frac{1}{l^{5 / 2}} \int_{0}^{l} h^{3 / 2} \iint \delta(t)\left|\eta(\alpha) u_{\alpha h}(\alpha, t)\right|^{2} d \alpha d t d h+K_{1} .
\end{aligned}
$$

We further note that

$$
\begin{aligned}
\partial_{\alpha}\left(\frac{\eta(\alpha) u_{h}(\alpha, t)}{z_{\alpha}(\alpha+h, t)}\right)= & \frac{\eta(\alpha) u_{\alpha h}(\alpha, t)}{z_{\alpha}(\alpha+h, t)}+\frac{\eta^{\prime}(\alpha) u_{h}(\alpha, t)}{z_{\alpha}(\alpha+h, t)} \\
& -\frac{\eta(\alpha) u_{h}(\alpha, t) z_{\alpha \alpha}(\alpha+h, t)}{z_{\alpha}^{2}(\alpha+h, t)}
\end{aligned}
$$

and 


$$
\begin{aligned}
\iint \delta(t)\left|\eta(\alpha) u_{h}(\alpha, t) z_{\alpha \alpha}(\alpha+h, t)\right|^{2} d \alpha d t & \\
& \leq \sup _{t \in \mathcal{T}} \delta(t)\left\|\eta u_{h}(\cdot, t)\right\|_{L^{2}(3 Q)}^{2} \int_{\mathcal{T}}\left\|\widetilde{z_{\alpha \alpha}(\cdot, t)}\right\|_{H^{1}(3 Q)}^{2} d t ;
\end{aligned}
$$

therefore for any $\epsilon>0$,

$$
\begin{aligned}
& \frac{1}{l^{5 / 2}} \int_{0}^{l} h^{3 / 2} \iint \delta(t)\left|\eta(\alpha) u_{t h}(\alpha, t)\right|^{2} d \alpha d t d h \\
& +\frac{1}{8 M^{4} l^{5 / 2}} \int_{0}^{l} h^{3 / 2} \iint \delta(t)\left|\eta(\alpha) u_{\alpha h}(\alpha, t)\right|^{2} d \alpha d t d h \\
& \quad \leq \frac{4 \epsilon}{l^{5 / 2}} \int_{0}^{l} h^{3 / 2} \iint \delta(t)\left|\eta(\alpha) u_{t h}(\alpha, t)\right|^{2} d \alpha d t d h \\
& \quad+4 c_{0}^{2} \varpi^{2}\left(z_{\alpha}, l\right) \frac{1}{l^{5 / 2}} \int_{0}^{l} h^{3 / 2} \iint \delta(t)\left|\eta(\alpha) u_{\alpha h}(\alpha, t)\right|^{2} d \alpha d t d h+K_{2}
\end{aligned}
$$

where $K_{2}<\infty$ is a constant depending on $m, M, 1 / \epsilon,|Q|,|\mathcal{T}|,\left\|z_{\alpha}\right\|_{H^{1}(3 Q \times \mathcal{T})}$, and $\left\|z_{\alpha \alpha}\right\|_{H^{1}(3 Q \times \mathcal{T})}$, and $K_{2}$ is independent of $l$. Notice further that

$$
\varpi\left(z_{\alpha}, l\right) \leq|l|^{1 / 2} \sup _{t \in \mathcal{T}}\left\|z_{\alpha \alpha}(\cdot, t)\right\|_{L^{2}(3 Q)} .
$$

Take $\epsilon$ small enough and let $l \rightarrow 0$. Since $Q, 3 Q \subset(a, b)$, is an arbitrarily small interval and $\mathcal{T} \subset(0, T)$ is arbitrary, we get

$$
\partial_{\alpha}^{2} z_{\alpha} \in H_{\mathrm{loc}}^{1}((a, b) \times(0, T)) .
$$

\section{$H^{5 / 2}$ Compatibility of the Initial Data}

We now prove the $H^{5 / 2}$ compatibility of the initial data. Take $\mathcal{T}=\left[0, T_{1}\right] \subset$ $[0, T)$. Let $\zeta(t)=\left(T_{1}-t\right)^{2}$ for $t \in \mathcal{T}$, and $\zeta(t)=0$ for $t \notin \mathcal{T}$. Instead of $\delta(t)$, we multiply (5.28) by $\zeta(t)$ and integrate with respect to $t$. Going through similar calculations as in (5.29) through (5.36), we arrive at

$$
\begin{aligned}
& \frac{1}{2 l^{5 / 2}} \int_{0}^{l} h^{3 / 2} \iint \zeta(t)\left|\eta(\alpha) u_{t h}(\alpha, t)\right|^{2} d \alpha d t d h \\
& +\frac{1}{16 M^{4} l^{5 / 2}} \int_{0}^{l} h^{3 / 2} \iint \zeta(t)\left|\eta(\alpha) u_{\alpha h}(\alpha, t)\right|^{2} d \alpha d t d h \\
& \leq \frac{\zeta(0)}{4 \pi l^{5 / 2}} \operatorname{Im} \int_{0}^{l} h^{3 / 2} \iint \frac{1}{(\alpha-\beta)^{2}}\left(\frac{\eta(\alpha) u_{h}(\alpha, 0)}{z_{\alpha}(\alpha+h, 0)}\right. \\
& \left.\quad-\frac{\eta(\beta) u_{h}(\beta, 0)}{z_{\beta}(\beta+h, 0)}\right)^{2} d \alpha d \beta d h+K_{3}
\end{aligned}
$$

where $K_{3}$ depends on $m, M,|Q|,|\mathcal{T}|,\left\|z_{\alpha}\right\|_{H^{1}(3 Q \times \mathcal{T})}$, and $\left\|z_{\alpha \alpha}\right\|_{H^{1}(3 Q \times \mathcal{T})}$, and $K_{3}$ is independent of $l$. Inequality (5.37) holds for $0<|l|<l_{0}$, where $l_{0}>0$ is a small enough constant. 
Recall $u=z_{\alpha \alpha}$. Notice that

$$
\begin{aligned}
\left(\partial_{\alpha} \ln z_{\alpha}(\alpha, 0)\right)_{h} & =\frac{z_{\alpha \alpha h}(\alpha, 0)}{z_{\alpha}(\alpha+h, 0)}-\frac{z_{\alpha \alpha}(\alpha, 0) z_{\alpha h}(\alpha, 0)}{z_{\alpha}(\alpha, 0) z_{\alpha}(\alpha+h, 0)} \\
& =\frac{u_{h}(\alpha, 0)}{z_{\alpha}(\alpha+h, 0)}-\frac{z_{\alpha \alpha}(\alpha, 0) z_{\alpha h}(\alpha, 0)}{z_{\alpha}(\alpha, 0) z_{\alpha}(\alpha+h, 0)} .
\end{aligned}
$$

Let $v(\alpha)=\operatorname{Re}\left((1+i) \ln z_{\alpha}(\alpha, 0)\right)$ and $w(\alpha)=\operatorname{Im}\left((1+i) \ln z_{\alpha}(\alpha, 0)\right)$. Therefore

$$
\begin{aligned}
& \zeta(0) \operatorname{Im} \iint \frac{1}{(\alpha-\beta)^{2}}\left(\frac{\eta(\alpha) u_{h}(\alpha, 0)}{z_{\alpha}(\alpha+h, 0)}-\frac{\eta(\beta) u_{h}(\beta, 0)}{z_{\beta}(\beta+h, 0)}\right)^{2} d \alpha d \beta \\
& \leq \quad \zeta(0) \operatorname{Im} \iint \frac{1}{(\alpha-\beta)^{2}}\left(\eta(\alpha)\left(\partial_{\alpha} \ln z_{\alpha}(\alpha, 0)\right)_{h}-\eta(\beta)\left(\partial_{\beta} \ln z_{\beta}(\beta, 0)\right)_{h}\right)^{2} d \alpha d \beta \\
& \left.\quad+\frac{\zeta(0)}{2} \iint \frac{1}{(\alpha-\beta)^{2}} \mid \eta(\alpha)\left(\partial_{\alpha} \ln z_{\alpha}(\alpha, 0)\right)_{h}-\eta(\beta)\left(\partial_{\beta} \ln z_{\beta}(\beta, 0)\right)_{h}\right)\left.\right|^{2} d \alpha d \beta \\
& \quad+3 \zeta(0) \iint \frac{1}{(\alpha-\beta)^{2}} \mid \frac{\eta(\alpha) z_{\alpha \alpha}(\alpha, 0) z_{\alpha h}(\alpha, 0)}{z_{\alpha}(\alpha, 0) z_{\alpha}(\alpha+h, 0)} \\
& \quad-\left.\frac{\eta(\beta) z_{\beta \beta}(\beta, 0) z_{\beta h}(\beta, 0)}{z_{\beta}(\beta, 0) z_{\beta}(\beta+h, 0)}\right|^{2} d \alpha d \beta \\
& =\frac{3 \zeta(0)}{4} \iint \frac{1}{(\alpha-\beta)^{2}}\left(\eta(\alpha) w_{\alpha h}(\alpha)-\eta(\beta) w_{\beta h}(\beta)\right)^{2} d \alpha d \beta \\
& \quad-\frac{\zeta(0)}{4} \iint \frac{1}{(\alpha-\beta)^{2}}\left(\eta(\alpha) v_{\alpha h}(\alpha)-\eta(\beta) v_{\beta h}(\beta)\right)^{2} d \alpha d \beta \\
& \quad+3 \zeta(0) \iint \frac{1}{(\alpha-\beta)^{2}} \mid \frac{\eta(\alpha) z_{\alpha \alpha}(\alpha, 0) z_{\alpha h}(\alpha, 0)}{z_{\alpha}(\alpha, 0) z_{\alpha}(\alpha+h, 0)} \\
& \quad-\left.\frac{\eta(\beta) z_{\beta \beta}(\beta, 0) z_{\beta h}(\beta, 0)}{z_{\beta}(\beta, 0) z_{\beta}(\beta+h, 0)}\right|^{2} d \alpha d \beta .
\end{aligned}
$$

We estimate

$$
\begin{aligned}
\zeta(0) & \iint \frac{1}{(\alpha-\beta)^{2}}\left|\frac{\eta(\alpha) z_{\alpha \alpha}(\alpha, 0) z_{\alpha h}(\alpha, 0)}{z_{\alpha}(\alpha, 0) z_{\alpha}(\alpha+h, 0)}-\frac{\eta(\beta) z_{\beta \beta}(\beta, 0) z_{\beta h}(\beta, 0)}{z_{\beta}(\beta, 0) z_{\beta}(\beta+h, 0)}\right|^{2} d \alpha d \beta \\
& =2 \zeta(0) \int \frac{\overline{\eta(\alpha) z_{\alpha \alpha}(\alpha, 0) z_{\alpha h}(\alpha, 0)}}{z_{\alpha}(\alpha, 0) z_{\alpha}(\alpha+h, 0)} H\left(\partial_{\alpha}\left(\frac{\eta(\alpha) z_{\alpha \alpha}(\alpha, 0) z_{\alpha h}(\alpha, 0)}{z_{\alpha}(\alpha, 0) z_{\alpha}(\alpha+h, 0)}\right)\right) d \alpha .
\end{aligned}
$$

We have

$$
\begin{gathered}
\zeta(0) \int \frac{\overline{\eta(\alpha) z_{\alpha \alpha}(\alpha, 0) z_{\alpha h}(\alpha, 0)}}{z_{\alpha}(\alpha, 0) z_{\alpha}(\alpha+h, 0)} H\left(\partial_{\alpha}\left(\frac{\eta(\alpha) z_{\alpha \alpha}(\alpha, 0) z_{\alpha h}(\alpha, 0)}{z_{\alpha}(\alpha, 0) z_{\alpha}(\alpha+h, 0)}\right)\right) d \alpha \\
=-\int \frac{d}{d t}\left(\zeta(t) \int \frac{\overline{\eta(\alpha) z_{\alpha \alpha}(\alpha, t) z_{\alpha h}(\alpha, t)}}{z_{\alpha}(\alpha, t) z_{\alpha}(\alpha+h, t)} H\left(\partial_{\alpha}\left(\frac{\eta(\alpha) z_{\alpha \alpha}(\alpha, t) z_{\alpha h}(\alpha, t)}{z_{\alpha}(\alpha, t) z_{\alpha}(\alpha+h, t)}\right)\right) d \alpha d t\right. \\
=-\int \zeta^{\prime}(t) \int \frac{\overline{\eta(\alpha) z_{\alpha \alpha}(\alpha, t) z_{\alpha h}(\alpha, t)}}{z_{\alpha}(\alpha, t) z_{\alpha}(\alpha+h, t)} H\left(\partial_{\alpha}\left(\frac{\eta(\alpha) z_{\alpha \alpha}(\alpha, t) z_{\alpha h}(\alpha, t)}{z_{\alpha}(\alpha, t) z_{\alpha}(\alpha+h, t)}\right)\right) d \alpha d t \\
-2 \operatorname{Re} \int \zeta(t) \int \partial_{t}\left(\frac{\eta(\alpha) z_{\alpha \alpha}(\alpha, t) z_{\alpha h}(\alpha, t)}{z_{\alpha}(\alpha, t) z_{\alpha}(\alpha+h, t)}\right) \\
\cdot H\left(\partial_{\alpha}\left(\frac{\eta(\alpha) z_{\alpha \alpha}(\alpha, t) z_{\alpha h}(\alpha, t)}{z_{\alpha}(\alpha, t) z_{\alpha}(\alpha+h, t)}\right)\right) d \alpha d t .
\end{gathered}
$$


Now

and

$$
\begin{aligned}
\partial_{t}( & \left.\frac{\eta(\alpha) z_{\alpha \alpha}(\alpha, t) z_{\alpha h}(\alpha, t)}{z_{\alpha}(\alpha, t) z_{\alpha}(\alpha+h, t)}\right) \\
= & \frac{\eta(\alpha) z_{t \alpha \alpha}(\alpha, t) z_{\alpha h}(\alpha, t)}{z_{\alpha}(\alpha, t) z_{\alpha}(\alpha+h, t)}+\frac{\eta(\alpha) z_{\alpha \alpha}(\alpha, t) z_{t \alpha h}(\alpha, t)}{z_{\alpha}(\alpha, t) z_{\alpha}(\alpha+h, t)} \\
& \quad-\frac{\eta(\alpha) z_{\alpha \alpha}(\alpha, t) z_{\alpha h}(\alpha, t)}{z_{\alpha}(\alpha, t) z_{\alpha}(\alpha+h, t)}\left(\frac{z_{t \alpha}(\alpha, t)}{z_{\alpha}(\alpha, t)}+\frac{z_{t \alpha}(\alpha+h, t)}{z_{\alpha}(\alpha+h, t)}\right)
\end{aligned}
$$

$$
\begin{aligned}
\partial_{\alpha}( & \left.\frac{\eta(\alpha) z_{\alpha \alpha}(\alpha, t) z_{\alpha h}(\alpha, t)}{z_{\alpha}(\alpha, t) z_{\alpha}(\alpha+h, t)}\right) \\
= & \frac{\eta(\alpha) z_{\alpha \alpha \alpha}(\alpha, t) z_{\alpha h}(\alpha, t)}{z_{\alpha}(\alpha, t) z_{\alpha}(\alpha+h, t)} \\
& +\frac{\eta^{\prime}(\alpha) z_{\alpha \alpha}(\alpha, t) z_{\alpha h}(\alpha, t)}{z_{\alpha}(\alpha, t) z_{\alpha}(\alpha+h, t)}+\frac{\eta(\alpha) z_{\alpha \alpha}(\alpha, t) z_{\alpha \alpha h}(\alpha, t)}{z_{\alpha}(\alpha, t) z_{\alpha}(\alpha+h, t)} \\
& -\frac{\eta(\alpha) z_{\alpha \alpha}(\alpha, t) z_{\alpha h}(\alpha, t)}{z_{\alpha}(\alpha, t) z_{\alpha}(\alpha+h, t)}\left(\frac{z_{\alpha \alpha}(\alpha, t)}{z_{\alpha}(\alpha, t)}+\frac{z_{\alpha \alpha}(\alpha+h, t)}{z_{\alpha}(\alpha+h, t)}\right)
\end{aligned}
$$

Using the estimates in (5.19), (5.29), and (5.30), the fact that

$$
\begin{aligned}
& \int \zeta(t)\left|\eta(\alpha) z_{\alpha \alpha}(\alpha, t) z_{t \alpha h}(\alpha, t)\right|^{2} d \alpha d t \\
& \quad \leq\left(\int_{3 Q}\left\|z_{\alpha \alpha}(\alpha, \cdot)\right\|_{L^{\infty}(\mathcal{T})}^{2} d \alpha\right) \sup _{\alpha \in Q}\left(\int \zeta(t)\left|\eta(\alpha) z_{t \alpha h}(\alpha, t)\right|^{2} d t\right),
\end{aligned}
$$

and Lemma 5.4, we have that for any $\epsilon>0$,

$$
\begin{aligned}
& \left|\zeta(0) \int \frac{\overline{\eta(\alpha) z_{\alpha \alpha}(\alpha, 0) z_{\alpha h}(\alpha, 0)}}{z_{\alpha}(\alpha, 0) z_{\alpha}(\alpha+h, 0)} H\left(\partial_{\alpha}\left(\frac{\eta(\alpha) z_{\alpha \alpha}(\alpha, 0) z_{\alpha h}(\alpha, 0)}{z_{\alpha}(\alpha, 0) z_{\alpha}(\alpha+h, 0)}\right)\right) d \alpha\right| \\
& \quad \leq \epsilon \iint \zeta(t)\left|\eta(\alpha) z_{\alpha \alpha t}(\alpha, t)\right|^{2} d \alpha d t+K_{4}
\end{aligned}
$$

where $K_{4}<\infty$ is independent of $h$, and $K_{4}$ depends on $1 / \epsilon, m, M,|Q|,|\mathcal{T}|$, $\left\|z_{\alpha}\right\|_{H^{1}(3 Q \times \mathcal{T})}$, and $\left\|z_{\alpha \alpha}\right\|_{H^{1}(3 Q \times \mathcal{T})}$.

From (5.37), (5.39), and (5.41) and with $\epsilon>0$ small enough, we conclude that

$$
\begin{aligned}
& \frac{1}{4 l^{5 / 2}} \int_{0}^{l} h^{3 / 2} \iint \zeta(t)\left|\eta(\alpha) u_{t h}(\alpha, t)\right|^{2} d \alpha d t d h \\
& +\frac{1}{16 M^{4} l^{5 / 2}} \int_{0}^{l} h^{3 / 2} \iint \zeta(t)\left|\eta(\alpha) u_{\alpha h}(\alpha, t)\right|^{2} d \alpha d t d h \\
& +\frac{|\mathcal{T}|^{2}}{16 \pi l^{5 / 2}} \int_{0}^{l} h^{3 / 2} \iint \frac{1}{(\alpha-\beta)^{2}}\left(\eta(\alpha) v_{\alpha h}(\alpha)-\eta(\beta) v_{\beta h}(\beta)\right)^{2} d \alpha d \beta d h \\
& \quad \leq \frac{3|\mathcal{T}|^{2}}{16 \pi l^{5 / 2}} \int_{0}^{l} h^{3 / 2} \iint \frac{1}{(\alpha-\beta)^{2}}\left(\eta(\alpha) w_{\alpha h}(\alpha)-\eta(\beta) w_{\beta h}(\beta)\right)^{2} d \alpha d \beta d h+K_{5}
\end{aligned}
$$

where $K_{5}<\infty$ depends on $m, M,|Q|,|\mathcal{T}|,\left\|z_{\alpha}\right\|_{H^{1}(3 Q \times \mathcal{T})}$, and $\left\|z_{\alpha \alpha}\right\|_{H^{1}(3 Q \times \mathcal{T})}$, and $K_{5}$ is independent of $l$. From the assumption that $w=\operatorname{Im}\left((1+i) \ln z_{\alpha}(\cdot, 0)\right) \in$ 
$H_{\mathrm{loc}}^{5 / 2}(a, b)$, we know the right-hand side of (5.42) is bounded by a constant independent of $l$. Let $l \rightarrow 0$. This proves that $v=\operatorname{Re}\left((1+i) \ln z_{\alpha}(\cdot, 0)\right) \in H_{\mathrm{loc}}^{5 / 2}(a, b)$ and $\partial_{\alpha}^{2} z_{\alpha} \in H_{\mathrm{loc}}^{1}((a, b) \times[0, T))$. The fact that $z_{\alpha}(\cdot, 0) \in H_{\mathrm{loc}}^{5 / 2}(a, b)$ directly follows from $\partial_{\alpha}^{2} z_{\alpha} \in H_{\mathrm{loc}}^{1}((a, b) \times[0, T))$.

\section{2 $H^{1}$ Regularity of $\partial_{\alpha}^{j} z_{\alpha}$ and $H^{j+1 / 2}$ Compatibility of the Initial Data, $j \geq 3$}

We prove by induction. Let $j \geq 3$. Assume that $z_{\alpha}, \ldots, \partial_{\alpha}^{j-1} z_{\alpha} \in H_{\text {loc }}^{1}((a, b) \times$ $(0, T))$ and that assumption (i) of Theorem 1.2 holds; we want to show $\partial_{\alpha}^{j} z_{\alpha} \in$ $H_{\mathrm{loc}}^{1}((a, b) \times(0, T))$. We also show that if, in addition, $\operatorname{Im}\left((1+i) \ln z_{\alpha}(\cdot, 0)\right) \in$ $H_{\mathrm{loc}}^{j+1 / 2}((a, b))$ and $z_{\alpha}, \ldots, \partial_{\alpha}^{j-1} z_{\alpha} \in H_{\mathrm{loc}}^{1}((a, b) \times[0, T))$, then

$$
\operatorname{Re}\left((1+i) \ln z_{\alpha}(\cdot, 0)\right) \in H_{\mathrm{loc}}^{j+1 / 2}((a, b)) \quad \text { and } \quad \partial_{\alpha}^{j} z_{\alpha} \in H_{\mathrm{loc}}^{1}((a, b) \times[0, T)) .
$$

The proof is very much the same as that for the case $j=2$ except that for $j \geq 3$ the proof is simpler because of the better regularity on $z_{\alpha}$ to begin with. We only sketch the proof, pointing out the differences.

Let $Q$ and $\mathcal{T}$ be compact intervals, $3 Q \subset(a, b)$ and $\mathcal{T} \subset(0, T)$. Let $\eta$ and $\rho$ be as in (5.13). Taking derivatives $\partial_{\alpha}^{j}$ on both sides of (1.6), using Lemmas 5.2 and 5.3, and distribution theory, we have, for $\alpha \in Q$,

$$
\partial_{\alpha}^{j} \bar{z}_{t}(\alpha, t)=\frac{1}{2 \pi i} \int \frac{-\partial_{\alpha}^{j} z(\alpha, t)+\partial_{\beta}^{j} z(\beta, t)}{(z(\alpha, t)-z(\beta, t))^{2}} \rho(\beta) d \beta+f_{3}(\alpha, t),
$$

where

$$
\begin{aligned}
& f_{3}(\alpha, t) \\
& =\frac{j !}{2 \pi i} \sum_{n=2}^{j}(-1)^{n} \int \frac{1}{(z(\alpha)-z(\beta))^{n+1}} \sum_{\substack{k_{1}+\cdots+k_{n}=j \\
k_{l} \geq 1}} \frac{\prod_{l=1}^{n}\left(\partial_{\alpha}^{k_{l}} z(\alpha)-\partial_{\beta}^{k_{l}} z(\beta)\right)}{k_{1} ! \cdots k_{n} !} \rho(\beta) d \beta \\
& \quad+\frac{1}{2 \pi i} \sum_{l=0}^{j-1}\left(\begin{array}{l}
l \\
j
\end{array}\right) \int\left(\partial_{\alpha}+\partial_{\beta}\right)^{l} \frac{1}{z(\alpha, t)-z(\beta, t)} \partial_{\beta}^{j-l} \rho(\beta) d \beta \\
& \quad+\frac{1}{2 \pi i} \int \partial_{\alpha}^{j} \frac{1}{z(\alpha, t)-z(\beta, t)}(1-\rho(\beta)) d \beta .
\end{aligned}
$$

Let

$$
u(\alpha, t)=\partial_{\alpha}^{j} z .
$$

Taking the difference quotient with respect to $\alpha$ on both sides of (5.43) and then multiplying by $\eta(\alpha)$, we get

$$
\eta(\alpha) \bar{u}_{t h}(\alpha, t)=\frac{\eta(\alpha)}{2 \pi i} \int \frac{-u_{h}(\alpha, t)+u_{h}(\beta, t)}{(z(\alpha+h, t)-z(\beta+h, t))^{2}} \rho(\beta) d \beta+f(\alpha, t)
$$


where

$$
\begin{aligned}
f(\alpha, t)= & \frac{\eta(\alpha)}{\pi i h} \int_{0}^{h} \int \frac{(u(\alpha, t)-u(\beta, t))\left(z_{\alpha}(\alpha+r, t)-z_{\beta}(\beta+r, t)\right)}{(z(\alpha+r, t)-z(\beta+r, t))^{3}} \rho(\beta) d \beta d r \\
& +\frac{\eta(\alpha)}{2 \pi i} \int \frac{-u(\alpha+h, t)+u(\beta+h, t)}{(z(\alpha+h, t)-z(\beta+h, t))^{2}} \rho_{h}(\beta) d \beta+\eta(\alpha) f_{3 h}(\alpha, t) .
\end{aligned}
$$

We note that the same discussion for (5.22), equality (5.23), and estimate (5.24) hold for $u=\partial_{\alpha}^{j} z$; therefore

$$
\begin{aligned}
\eta(\alpha) \bar{u}_{t h}= & \frac{1}{2 \pi i z_{\alpha}(\alpha+h, t)} \int \frac{1}{(\alpha-\beta)^{2}}\left(\frac{\eta(\beta) u_{h}(\beta, t)}{z_{\beta}(\beta+h, t)}-\frac{\eta(\alpha) u_{h}(\alpha, t)}{z_{\alpha}(\alpha+h, t)}\right) d \beta \\
& +G_{1}(\alpha, t)
\end{aligned}
$$

where

$$
G_{1}(\alpha, t)=-\frac{1}{2 \pi i} \eta(\alpha) u_{h}(\alpha, t) F(\alpha, t)+\frac{1}{2 \pi i} A_{2}+\frac{1}{2 \pi i} B+f(\alpha, t) .
$$

\section{$H_{\text {loc }}^{1}$ Regularity of $\partial_{\alpha}^{j} z_{\alpha}$}

Assume $\mathcal{T}=\left[T_{1}, T_{2}\right] \subset(0, T)$. Let $\delta(t)=\left(t-T_{1}\right)^{2}\left(T_{2}-t\right)^{2}$ for $t \in \mathcal{T}$ and $\delta(t)=0$ for $t \notin \mathcal{T}$. We estimate $f_{3 h}$ through $\partial_{\alpha} f_{3}$, the fact that

$$
f_{3 h}(\alpha, t)=\frac{1}{h} \int_{0}^{h} \partial_{\alpha} f_{3}(\alpha+r, t) d r
$$

$\operatorname{supp} \eta \subset Q, \rho^{\prime}(\beta)=0$ for $\beta \in \frac{3}{2} Q, \rho_{h}(\beta)=0$ for $\beta \in \frac{11}{8} Q$ and $0<|h| \leq \frac{1}{8}|Q|$, $1-\rho(\beta)=0$ for $\beta \in \frac{3}{2} Q$, and Lemmas 5.2, 5.3, 5.4, 5.5, and 5.6. We estimate the remaining terms in $G_{1}(\alpha, t)$ by Lemma 5.6 and (5.24). We obtain, for $0<|h| \leq$ $\frac{1}{8}|Q|$,

$$
\iint \delta(t)\left|G_{1}(\alpha, t)\right|^{2} d \alpha d t \leq K_{6}<\infty
$$

where $K_{6}$ depends on $m, M,|Q|,|\mathcal{T}|$, and $\left\|\partial_{\alpha}^{k} z_{\alpha}\right\|_{H^{1}(3 Q \times \mathcal{T})}, k=0, \ldots, j-1$; in particular, $K_{6}$ is independent of $h$. Notice that (5.28) holds for any $u$ satisfying (5.25), and (5.33) holds for any $u$. Moreover, by taking the derivative with respect to $t$ of equation (5.14) and using Lemma 5.6, we have

$$
z_{t t \alpha \alpha} \in L^{2}(3 Q \times \mathcal{T})
$$

and $z_{t t \alpha} \in L^{2}(3 Q \times \mathcal{T})$ can be obtained by taking the derivatives $\partial_{t} \partial_{\alpha}$ of (1.6) and Lemma 5.6. We get from (5.28), (5.33), and (5.47) that for $0<|h|<\frac{1}{8}|Q|$,

$$
\begin{aligned}
& \iint \delta(t)\left|\eta(\alpha) u_{t h}(\alpha, t)\right|^{2} d \alpha d t+\frac{1}{8 M^{2}} \iint \delta(t)\left|\partial_{\alpha}\left(\frac{\eta(\alpha) u_{h}(\alpha, t)}{z_{\alpha}(\alpha+h, t)}\right)\right|^{2} d \alpha d t \\
& \quad \leq K_{7}<\infty
\end{aligned}
$$

where $K_{7}$ depends on $m, M,|Q|,|\mathcal{T}|,\left\|\partial_{\alpha}^{k} z_{\alpha}\right\|_{H^{1}(3 Q \times \mathcal{T})}, k=0, \ldots, j-1$,

$$
\left\|z_{t t \alpha \alpha}\right\|_{L^{2}(3 Q \times \mathcal{T})}, \quad \text { and }\left\|z_{t \alpha \alpha}\right\|_{L^{2}(3 Q \times \mathcal{T})} \text {. }
$$


The constant $K_{7}$ is independent of $h$. Let $h \rightarrow 0$. We conclude

$$
\partial_{\alpha}^{j} z_{\alpha} \in H_{\mathrm{loc}}^{1}((a, b) \times(0, T)) .
$$

\section{$H^{j+1 / 2}$ Compatibility of the Initial Data, $j \geq 3$}

Assume that $\operatorname{Im}\left((1+i) \ln z_{\alpha}(\cdot, 0)\right) \in H_{\mathrm{loc}}^{j+1 / 2}(a, b)$ and

$$
z_{\alpha}(\cdot, 0) \in H_{\mathrm{loc}}^{j-1 / 2}(a, b), \quad z_{\alpha}, \ldots, \partial_{\alpha}^{j-1} z_{\alpha} \in H_{\mathrm{loc}}^{1}((a, b) \times[0, T)) .
$$

We take $\mathcal{T}=\left[0, T_{1}\right] \subset[0, T)$. Let $\zeta(t)=\left(T_{1}-t\right)^{2}$ for $t \in \mathcal{T}$, and $\zeta(t)=0$ for $t \notin \mathcal{T}$. Similarly to the case $j=2$, we have

$$
\begin{aligned}
& \iint \zeta(t)\left|\eta(\alpha) u_{t h}(\alpha, t)\right|^{2} d \alpha d t+\frac{1}{8 M^{2}} \iint \zeta(t)\left|\partial_{\alpha}\left(\frac{\eta(\alpha) u_{h}(\alpha, t)}{z_{\alpha}(\alpha+h, t)}\right)\right|^{2} d \alpha d t \\
& \quad \leq \frac{\zeta(0)}{4 \pi} \operatorname{Im} \iint \frac{1}{(\alpha-\beta)^{2}}\left(\frac{\eta(\alpha) u_{h}(\alpha, 0)}{z_{\alpha}(\alpha+h, 0)}-\frac{\eta(\beta) u_{h}(\beta, 0)}{z_{\beta}(\beta+h, 0)}\right)^{2} d \alpha d \beta+K_{8}
\end{aligned}
$$

where $K_{8}<\infty$ depends on $m, M,|Q|,|\mathcal{T}|,\left\|\partial_{\alpha}^{k} z_{\alpha}\right\|_{H^{1}(3 Q \times \mathcal{T})}, k=0, \ldots, j-1$, $\left\|z_{t t \alpha \alpha}\right\|_{L^{2}(3 Q \times \mathcal{T})}$, and $\left\|z_{t \alpha \alpha}\right\|_{L^{2}(3 Q \times \mathcal{T})}$, and is independent of $h$.

Notice that

$$
\left(\partial_{\alpha}^{j-1} \ln z_{\alpha}(\alpha, 0)\right)_{h}=\frac{u_{h}(\alpha, 0)}{z_{\alpha}(\alpha+h, 0)}+\mathcal{R}(\alpha)
$$

The fact that $\mathcal{R} \in H_{\text {loc }}^{1 / 2}$ with $\left\||D|^{1 / 2}(\eta \mathcal{R})\right\|_{L^{2}(\mathbb{R})}<K_{9}$, where $K_{9}<\infty$ is independent of $h$, can be obtained directly from the inductive assumption $z_{\alpha}(\cdot, 0) \in$ $H_{\mathrm{loc}}^{j-1 / 2}(a, b)$ and Lemma 5.9. Following through the same argument as in the case $j=2$, we conclude that $\operatorname{Re}(1+i) \ln z_{\alpha}(\cdot, 0) \in H_{\mathrm{loc}}^{j+1 / 2}(a, b), \partial_{\alpha}^{j} z_{\alpha} \in$ $H_{\mathrm{loc}}^{1}((a, b) \times[0, T))$, and $z_{\alpha}(\cdot, 0) \in H_{\mathrm{loc}}^{j+1 / 2}(a, b)$ for $j \geq 3$.

\section{Analyticity}

In this section, we prove the analyticity of $z_{\alpha}(\cdot, t)$ on $(a, b)$ for fixed $t \in$ $(0, T)$ under the assumptions of Theorem 1.2. We have so far obtained $\partial_{\alpha}^{j} z_{\alpha} \in$ $H_{\mathrm{loc}}^{1}((a, b) \times(0, T))$ for all $j \geq 0$. We also show that if, in addition, $\operatorname{Im}((1+$ $\left.i) \ln z_{\alpha}(\cdot, 0)\right)$ is analytic on $(a, b)$, then $\operatorname{Re}\left((1+i) \ln z_{\alpha}(\cdot, 0)\right)$ is analytic on $(a, b)$.

We first consider the analyticity of $z_{\alpha}(\cdot, t)$ for fixed $t \in(0, T)$. Let $Q$ and $\mathcal{T}$ be compact intervals, $3 Q \subset(a, b), \mathcal{T} \subset(0, T)$. Let $\eta \in C^{\infty}(Q) \cap C_{0}^{3}(\mathbb{R})$ and $\rho \in C_{0}^{\infty}(\mathbb{R})$ such that $\operatorname{supp} \eta \subset Q$, supp $\rho \subset 3 Q, \rho(\alpha)=1$ for $\alpha \in 2 Q$, and $\eta(\alpha), \rho(\alpha) \geq 0$ for $\alpha \in \mathbb{R}$. We will later give a specific construction of $\eta$ when it becomes clear what is required of it. 
Let $j \geq 1$. Taking derivatives $\left(\eta(\alpha) \partial_{\alpha}\right)^{j}$ on both sides of (1.6) and using Lemmas 5.2 and 5.3, we get

$$
\begin{array}{r}
\left(\eta(\alpha) \partial_{\alpha}\right)^{j} \bar{z}_{t}(\alpha, t)+\frac{1}{2 \pi i} \int \frac{\left(\eta(\alpha) \partial_{\alpha}\right)^{j} z(\alpha, t)-\left(\eta(\beta) \partial_{\beta}\right)^{j} z(\beta, t)}{(z(\alpha, t)-z(\beta, t))^{2}} d \beta \\
=\frac{j !}{2 \pi i} A+\frac{j !}{2 \pi i} B
\end{array}
$$

where

$$
\begin{aligned}
A=\sum_{n=2}^{j}(-1)^{n} \int & \frac{1}{(z(\alpha, t)-z(\beta, t))^{n+1}} \\
& \cdot \sum_{\substack{k_{1}+\cdots+k_{n}=j \\
k_{l} \geq 1}} \frac{\prod_{l=1}^{n}\left(\left(\eta(\alpha) \partial_{\alpha}\right)^{k_{l}} z(\alpha, t)-\left(\eta(\beta) \partial_{\beta}\right)^{k_{l}} z(\beta, t)\right)}{k_{1} ! \cdots k_{n} !} d \beta
\end{aligned}
$$

(when $j=1$ and $A=0$ ) and

$$
B=\sum_{l=0}^{j-1} \frac{1}{l !(j-l) !} \int\left(\eta(\alpha) \partial_{\alpha}+\eta(\beta) \partial_{\beta}\right) \frac{1}{z(\alpha, t)-z(\beta, t)}\left(\partial_{\beta} \eta(\beta)\right)^{j-l} 1 d \beta
$$

We take further derivatives $\left(\rho(\alpha) \partial_{\alpha}\right)^{2}$ of (6.1). Notice that $\left(\rho(\alpha) \partial_{\alpha}\right)^{\iota}\left(\eta(\alpha) \partial_{\alpha}\right)^{k}=$ $\partial_{\alpha}^{\iota}\left(\eta(\alpha) \partial_{\alpha}\right)^{k}$ for $k \geq 1$. We may calculate $\left(\rho(\alpha) \partial_{\alpha}\right)^{2} A$ and $\left(\rho(\alpha) \partial_{\alpha}\right)^{2} B$ using formula (5.2). On the other hand, taking $\left(\rho(\alpha) \partial_{\alpha}\right)^{2}$ on the left-hand side of (6.1) and using (5.2), we get

$$
\begin{aligned}
& \left(\rho(\alpha) \partial_{\alpha}\right)^{2}\left(\left(\eta(\alpha) \partial_{\alpha}\right)^{j} \bar{z}_{t}(\alpha, t)+\frac{1}{2 \pi i} \int \frac{\left(\eta(\alpha) \partial_{\alpha}\right)^{j} z(\alpha, t)-\left(\eta(\beta) \partial_{\beta}\right)^{j} z(\beta, t)}{(z(\alpha, t)-z(\beta, t))^{2}} d \beta\right) \\
& =\partial_{\alpha}^{2}\left(\eta(\alpha) \partial_{\alpha}\right)^{j} \bar{z}_{t}(\alpha, t)+\frac{1}{2 \pi i} \int \frac{\partial_{\alpha}^{2}\left(\eta(\alpha) \partial_{\alpha}\right)^{j} z(\alpha, t)-\partial_{\beta}^{2}\left(\eta(\beta) \partial_{\beta}\right)^{j} z(\beta, t)}{(z(\alpha, t)-z(\beta, t))^{2}} d \beta \\
& \quad+\frac{j !}{2 \pi i} \mathcal{D}
\end{aligned}
$$

where

$$
\begin{aligned}
j ! \mathcal{D}= & -4 \int \frac{\left(\partial_{\alpha}\left(\eta(\alpha) \partial_{\alpha}\right)^{j} z(\alpha, t)-\partial_{\beta}\left(\eta(\beta) \partial_{\beta}\right)^{j} z(\beta, t)\right)\left(\rho(\alpha) z_{\alpha}(\alpha, t)-\rho(\beta) z_{\beta}(\beta, t)\right)}{(z(\alpha, t)-z(\beta, t))^{3}} d \beta \\
& -2 \int \frac{\left(\left(\eta(\alpha) \partial_{\alpha}\right)^{j} z(\alpha, t)-\left(\eta(\beta) \partial_{\beta}\right)^{j} z(\beta, t)\right)\left(\left(\rho(\alpha) \partial_{\alpha}\right)^{2} z(\alpha, t)-\left(\rho(\beta) \partial_{\beta}\right)^{2} z(\beta, t)\right)}{(z(\alpha, t)-z(\beta, t))^{3}} d \beta \\
& +6 \int \frac{\left(\left(\eta(\alpha) \partial_{\alpha}\right)^{j} z(\alpha, t)-\left(\eta(\beta) \partial_{\beta}\right)^{j} z(\beta, t)\right)\left(\rho(\alpha) z_{\alpha}(\alpha, t)-\rho(\beta) z_{\beta}(\beta, t)\right)^{2}}{(z(\alpha, t)-z(\beta, t))^{4}} d \beta \\
& +2 \int \frac{\partial_{\alpha}\left(\eta(\alpha) \partial_{\alpha}\right)^{j} z(\alpha, t)-\partial_{\beta}\left(\eta(\beta) \partial_{\beta}\right)^{j} z(\beta, t)}{(z(\alpha, t)-z(\beta, t))^{2}} \rho^{\prime}(\beta) d \beta
\end{aligned}
$$




$$
\begin{aligned}
& -4 \int \frac{\left(\left(\eta(\alpha) \partial_{\alpha}\right)^{j} z(\alpha, t)-\left(\eta(\beta) \partial_{\beta}\right)^{j} z(\beta, t)\right)\left(\rho(\alpha) z_{\alpha}(\alpha, t)-\rho(\beta) z_{\beta}(\beta, t)\right)}{(z(\alpha, t)-z(\beta, t))^{3}} \rho^{\prime}(\beta) d \beta \\
& +\int \frac{\left(\eta(\alpha) \partial_{\alpha}\right)^{j} z(\alpha, t)-\left(\eta(\beta) \partial_{\beta}\right)^{j} z(\beta, t)}{(z(\alpha, t)-z(\beta, t))^{2}} \partial_{\beta}\left(\rho(\beta) \rho^{\prime}(\beta)\right) d \beta .
\end{aligned}
$$

Therefore

$$
\begin{aligned}
& \partial_{\alpha}^{2}\left(\eta(\alpha) \partial_{\alpha}\right)^{j} \bar{z}_{t}(\alpha, t) \\
& +\frac{1}{2 \pi i} \int \frac{\partial_{\alpha}^{2}\left(\eta(\alpha) \partial_{\alpha}\right)^{j} z(\alpha, t)-\partial_{\beta}^{2}\left(\eta(\beta) \partial_{\beta}\right)^{j} z(\beta, t)}{(z(\alpha, t)-z(\beta, t))^{2}} d \beta \\
& \quad=-\frac{j !}{2 \pi i} \mathcal{D}+\frac{j !}{2 \pi i}\left(\rho(\alpha) \partial_{\alpha}\right)^{2} A+\frac{j !}{2 \pi i}\left(\rho(\alpha) \partial_{\alpha}\right)^{2} B
\end{aligned}
$$

Assume $\mathcal{T}=\left[T_{1}, T_{2}\right]$. Let $\sigma(t)=\left(T_{2}-t\right)\left(t-T_{1}\right)$ for $t \in \mathcal{T}$ and $\sigma(t)=0$ for $t \notin \mathcal{T}$. We want to obtain estimates for

$$
\left\|\sigma^{j}\left(\rho \partial_{\alpha}\right)^{2} A\right\|_{L^{2}(R \times \mathcal{T})}=\left(\iint \sigma^{2 j}(t)\left|\left(\rho \partial_{\alpha}\right)^{2} A\right|^{2} d \alpha d t\right)^{1 / 2},
$$

$\left\|\sigma^{j}\left(\rho \partial_{\alpha}\right)^{2} B\right\|_{L^{2}(R \times \mathcal{T})}$, and $\left\|\sigma^{j} \mathcal{D}\right\|_{L^{2}(R \times \mathcal{T})}$. Notice that from $\sigma\left(T_{2}\right)=0$, we have, for $k \geq 1$,

$$
\begin{aligned}
\sup _{t \in \mathcal{T}} & \left\|\sigma^{k}(t) \partial_{\alpha}^{2}\left(\eta \partial_{\alpha}\right)^{k} z(\cdot, t)\right\|_{L^{2}(\mathbb{R})}^{2} \\
\leq & \int\left|\partial_{t}\left(\left\|\sigma^{k}(t) \partial_{\alpha}^{2}\left(\eta \partial_{\alpha}\right)^{k} z(\cdot, t)\right\|_{L^{2}(\mathbb{R})}^{2}\right)\right| d t \\
\leq & 2 k \iint \sigma^{2 k-1}(t)\left|\sigma^{\prime}(t)\right|\left|\partial_{\alpha}^{2}\left(\eta \partial_{\alpha}\right)^{k} z(\alpha, t)\right|^{2} d \alpha d t \\
& +2 \iint \sigma^{2 k}(t)\left|\partial_{\alpha}^{2}\left(\eta \partial_{\alpha}\right)^{k} z(\alpha, t)\right|\left|\partial_{\alpha}^{2}\left(\eta \partial_{\alpha}\right)^{k} z_{t}(\alpha, t)\right| d \alpha d t \\
\leq & \left(2|\mathcal{T}|^{3} k+|\mathcal{T}|^{4}\right)\left\|\sigma^{k-1} \partial_{\alpha}^{2}\left(\eta \partial_{\alpha}\right)^{k} z\right\|_{L^{2}(3 Q \times \mathcal{T})}^{2} \\
& +\left\|\sigma^{k} \partial_{\alpha}^{2}\left(\eta \partial_{\alpha}\right)^{k} z_{t}\right\|_{L^{2}(3 Q \times \mathcal{T})}^{2} .
\end{aligned}
$$

Here we used the fact that $|\sigma(t)| \leq|\mathcal{T}|^{2}$ and $\left|\sigma^{\prime}(t)\right| \leq|\mathcal{T}|$. Furthermore, for $k \geq 1$,

$$
\left\|\partial_{\alpha}^{2}\left(\eta \partial_{\alpha}\right)^{k+1} z(\cdot, t)\right\|_{L^{2}(3 Q)} \leq c_{1}\left\|\partial_{\alpha}^{3}\left(\eta \partial_{\alpha}\right)^{k} z(\cdot, t)\right\|_{L^{2}(3 Q)},
$$

where $c_{1}=\left\|\eta^{\prime \prime}\right\|_{\infty}|Q|^{2}+2\left\|\eta^{\prime}\right\|_{\infty}|Q|+\|\eta\|_{\infty}$.

Let

$$
\begin{aligned}
& a_{k}=\left\|\sigma^{k} \partial_{\alpha}^{3}\left(\eta \partial_{\alpha}\right)^{k} z\right\|_{L^{2}(3 Q \times \mathcal{T})}, \\
& b_{k}=\left\|\sigma^{k} \partial_{\alpha}^{2}\left(\eta \partial_{\alpha}\right)^{k} z_{t}\right\|_{L^{2}(3 Q \times \mathcal{T})}, \\
& d_{k}=|\mathcal{T}|^{2 k}\left\|\partial_{\alpha}^{2}\left(\partial_{\alpha} \eta\right)^{k} 1\right\|_{L^{2}(3 Q)},
\end{aligned}
$$

for $k \geq 1$ and $a_{0}=\left\|\partial_{\alpha}^{2}\left(\eta z_{\alpha}\right)\right\|_{L^{2}(3 Q \times \mathcal{T})}$. 
We now estimate $\left\|\sigma^{j}\left(\rho \partial_{\alpha}\right)^{2} A\right\|_{L^{2}(\mathbb{R} \times \mathcal{T})}$. Using (5.2), we have

$$
\begin{aligned}
& \left(\rho(\alpha) \partial_{\alpha}\right)^{2} A \\
& =\sum_{n=2}^{j}(-1)^{n} \sum_{\substack{k_{1}+\ldots+k_{n}=j \\
k_{l} \geq 1}} \int\left(\rho(\alpha) \partial_{\alpha}+\rho(\beta) \partial_{\beta}\right)^{2} \\
& \cdot \frac{\prod_{l=1}^{n}\left(\left(\eta(\alpha) \partial_{\alpha}\right)^{k_{l}} z(\alpha, t)-\left(\eta(\beta) \partial_{\beta}\right)^{k_{l}} z(\beta, t)\right)}{(z(\alpha, t)-z(\beta, t))^{n+1} k_{1} ! \cdots k_{n} !} d \beta \\
& +2 \sum_{n=2}^{j}(-1)^{n} \sum_{\substack{k_{1}+\ldots+k_{n}=j \\
k_{l} \geq 1}} \int\left(\rho(\alpha) \partial_{\alpha}+\rho(\beta) \partial_{\beta}\right) \\
& \cdot \frac{\prod_{l=1}^{n}\left(\left(\eta(\alpha) \partial_{\alpha}\right)^{k_{l}} z(\alpha, t)-\left(\eta(\beta) \partial_{\beta}\right)^{k_{l}} z(\beta, t)\right)}{(z(\alpha, t)-z(\beta, t))^{n+1} k_{1} ! \cdots k_{n} !} \rho^{\prime}(\beta) d \beta \\
& \begin{aligned}
+\sum_{n=2}^{j}(-1)^{n} \sum_{\substack{k_{1}+\ldots+k_{n}=j \\
k_{l} \geq 1}} \int \frac{\prod_{l=1}^{n}\left(\left(\eta(\alpha) \partial_{\alpha}\right)^{k_{l}} z(\alpha, t)-\left(\eta(\beta) \partial_{\beta}\right)^{k_{l}} z(\beta, t)\right)}{(z(\alpha, t)-z(\beta, t))^{n+1} k_{1} ! \cdots k_{n} !} \\
. \partial_{\beta}\left(\rho(\beta) \rho^{\prime}(\beta)\right) d \beta,
\end{aligned}
\end{aligned}
$$

Further expanding the derivatives

$$
\left(\rho(\alpha) \partial_{\alpha}+\rho(\beta) \partial_{\beta}\right)^{l} \frac{\prod_{l=1}^{n}\left(\left(\eta(\alpha) \partial_{\alpha}\right)^{k_{l}} z(\alpha, t)-\left(\eta(\beta) \partial_{\beta}\right)^{k_{l}} z(\beta, t)\right)}{(z(\alpha, t)-z(\beta, t))^{n+1} k_{1} ! \cdots k_{n} !},
$$

$\iota=1,2$, we then apply Lemma 5.6(ii), Remark 5.7, Lemma 5.5, (6.3), and (6.4). We obtain the following estimate:

$$
\left\|\sigma^{j}\left(\rho \partial_{\alpha}\right)^{2} A\right\|_{L^{2}(R \times \mathcal{T})} \leq \sum_{n=2}^{j} \sum_{\substack { k_{1} \\
\begin{subarray}{c}{k_{l} \geq 1 \\
k_{\iota} \geq 1{ k _ { 1 } \\
\begin{subarray} { c } { k _ { l } \geq 1 \\
k _ { \iota } \geq 1 } }\end{subarray}} c_{0}^{n} n^{2} \frac{\prod_{l=1}^{n}\left(a_{k_{\iota}}+b_{k_{\iota}}+v k_{\iota} a_{k_{\iota}-1}\right)}{k_{1} ! \cdots k_{n} !}
$$

where $c_{0}, v>0$ are constants, $c_{0}$ depending on $\left\|z_{\alpha}\right\|_{H^{1}(3 Q \times \mathcal{T})},\left\|z_{\alpha \alpha}\right\|_{H^{1}(3 Q \times \mathcal{T})}$, $m, M,|Q|,|\mathcal{T}|$, and $\rho$, and $v$ depending on $c_{1}$ and $|\mathcal{T}|$. Similarly, expanding the terms in $\left(\rho(\alpha) \partial_{\alpha}\right)^{2} B$ according to Lemmas 5.3 and 5.2 and applying Lemma 5.6(ii), Remark 5.7, Lemma 5.5, (6.3), and (6.4), we have, for the same constants $c_{0}$ and $v$ as in (6.6) (we can make them the same by choosing the bigger of the two), that

$$
\begin{aligned}
& \left\|\sigma^{j}\left(\rho \partial_{\alpha}\right)^{2} B\right\|_{L^{2}(R \times \mathcal{T})} \\
& \leq \sum_{l=1}^{j-1} \sum_{n=1}^{l} \sum_{\substack{k_{1}+\cdots+k_{n}=l \\
k_{l} \geq 1}} c_{0}^{n+1}(n+1)^{2} \frac{\prod_{l=1}^{n}\left(a_{k_{l}}+b_{k_{l}}+v k_{l} a_{k_{l}-1}\right)}{k_{1} ! \cdots k_{n} !} \frac{d_{j-l}}{(j-l) !} \\
& \quad+c_{0} \frac{d_{j}}{j !} .
\end{aligned}
$$

Furthermore, applying Lemmas 5.6 and 5.5 and then (6.4) to $\mathcal{D}$, we get

$$
\left\|\sigma^{j} \mathcal{D}\right\|_{L^{2}(R \times \mathcal{T})} \leq c_{2} \frac{1}{j !}\left\|\sigma^{j} \partial_{\alpha}^{2}\left(\eta \partial_{\alpha}\right)^{j} z\right\|_{L^{2}(3 Q \times \mathcal{T})} \leq c_{2}^{\prime} \frac{1}{(j-1) !} a_{j-1},
$$


where $c_{2}$ and $c_{2}^{\prime}$ depend on $\left\|z_{\alpha}\right\|_{H^{1}(3 Q \times \mathcal{T})},\left\|z_{\alpha \alpha}\right\|_{H^{1}(3 Q \times \mathcal{T})},\left\|z_{\alpha \alpha \alpha}\right\|_{H^{1}(3 Q \times \mathcal{T})},|Q|$, $|\mathcal{T}|, m, M$, and $\rho$.

We now consider the left-hand side of (6.2). Let

$$
u=\partial_{\alpha}^{2}\left(\eta(\alpha) \partial_{\alpha}\right)^{j} z(\alpha, t)
$$

We may handle the term

$$
\frac{1}{2 \pi i} \int \frac{u(\alpha, t)-u(\beta, t)}{(z(\alpha, t)-z(\beta, t))^{2}} d \beta
$$

in the same way as in Section 5 by rewriting it as

$$
\frac{1}{2 \pi i} \int \frac{u(\alpha, t)-u(\beta, t)}{(z(\alpha, t)-z(\beta, t))^{2}} d \beta=\frac{1}{2 i z_{\alpha}(\alpha, t)} H\left(\partial_{\alpha}\left(\frac{u(\alpha, t)}{z_{\alpha}(\alpha, t)}\right)\right)+\frac{j !}{2 \pi i} E
$$

where

$$
\begin{aligned}
j ! E= & u(\alpha, t) \int \frac{z_{\beta}(\beta, t)}{(z(\alpha, t)-z(\beta, t))^{2}}\left(\frac{\rho(\beta)}{z_{\beta}(\beta, t)}-\frac{\rho(\alpha)}{z_{\alpha}(\alpha, t)}\right) d \beta \\
& +\int \frac{u(\alpha, t)}{(z(\alpha, t)-z(\beta))^{2}}(1-\rho(\beta)) d \beta \\
& -\frac{1}{z_{\alpha}(\alpha, t)} \int\left(\frac{z_{\beta}(\beta, t) z_{\alpha}(\alpha, t)}{z(\alpha, t)-z(\beta, t)^{2}}-\frac{1}{(\alpha-\beta)^{2}}\right) \frac{u(\beta, t)}{z_{\beta}(\beta, t)} d \beta .
\end{aligned}
$$

Similarly to (5.24), we apply Lemma 5.6 to the first term and the T1 theorem to the third term in $E$ while estimating the second term in $E$ straightforwardly using the fact $\rho(\beta)=1$ on $2 Q$. We get

$$
\left\|\sigma^{j} E\right\|_{L^{2}(R \times \mathcal{T})} \leq c_{3} \frac{1}{j !}\left\|\sigma^{j} \partial_{\alpha}^{2}\left(\eta \partial_{\alpha}\right)^{j} z\right\|_{L^{2}(3 Q \times \mathcal{T})} \leq c_{3}^{\prime} \frac{1}{(j-1) !} a_{j-1},
$$

where $c_{3}$ and $c_{3}^{\prime}$ depend on $\left\|z_{\alpha}\right\|_{H^{1}(3 Q \times \mathcal{T})},\left\|z_{\alpha \alpha}\right\|_{H^{1}(3 Q \times \mathcal{T})},\left\|z_{\alpha \alpha \alpha}\right\|_{H^{1}(3 Q \times \mathcal{T})},|Q|$, $|\mathcal{T}|, m, M$, and $\rho$. Let

$$
\mathcal{M}=\bar{u}_{t}(\alpha, t)+\frac{1}{2 i z_{\alpha}(\alpha, t)} H\left(\partial_{\alpha}\left(\frac{u(\alpha, t)}{z_{\alpha}(\alpha, t)}\right)\right) .
$$

From a similar argument to that in (5.27), (5.28), and (5.33), we obtain

$$
\begin{aligned}
\left\|\sigma^{j} u_{t}\right\|_{L^{2}(R \times \mathcal{T})}^{2}+ & \frac{1}{8 M^{4}}\left\|\sigma^{j} u_{\alpha}\right\|_{L^{2}(R \times \mathcal{T})}^{2} \\
& \leq c_{4} j^{2}\left\|\sigma^{j-1} \partial_{\alpha}^{2}\left(\eta \partial_{\alpha}\right)^{j} z\right\|_{L^{2}(3 Q \times \mathcal{T})}^{2}+\left\|\sigma^{j} \mathcal{M}\right\|_{L^{2}(R \times \mathcal{T})}^{2},
\end{aligned}
$$

where $c_{4}$ depends on $\left\|z_{\alpha}\right\|_{H^{1}(3 Q \times \mathcal{T})},\left\|z_{\alpha \alpha}\right\|_{H^{1}(3 Q \times \mathcal{T})},\left\|z_{t \alpha}\right\|_{H^{2}(3 Q \times \mathcal{T})},|Q|,|\mathcal{T}|, m$, $M$, and $\rho$. We know from (6.2), (6.10), and (6.12) that

$$
\mathcal{M}=\frac{j !}{2 \pi i}\left(-E-\mathcal{D}+\left(\rho(\alpha) \partial_{\alpha}\right)^{2} A+\left(\rho(\alpha) \partial_{\alpha}\right)^{2} B\right) ;
$$


using estimates (6.6), (6.7), (6.8), (6.11), and (6.13), we arrive at

$$
\begin{aligned}
& \frac{1}{4 M^{2}} \frac{a_{j}}{j !}+\frac{b_{j}}{j !} \\
& \leq c_{0}^{\prime} \frac{a_{j-1}}{(j-1) !}+c_{0} \frac{d_{j}}{j !}+\sum_{n=2}^{j} \sum_{\substack{k_{1}+\cdots+k_{n}=j \\
k_{l} \geq 1}} c_{0}^{n} n^{2} \frac{\prod_{l=1}^{n}\left(a_{k_{\iota}}+b_{k_{\iota}}+v k_{\iota} a_{k_{l}-1}\right)}{k_{1} ! \cdots k_{n} !} \\
& \quad+\sum_{l=1}^{j-1} \sum_{n=1}^{l} \sum_{\substack{k_{1}+\ldots+k_{n}=l \\
k_{l} \geq 1}} c_{0}^{n+1}(n+1)^{2} \frac{\prod_{l=1}^{n}\left(a_{k_{\iota}}+b_{k_{\iota}}+v k_{\iota} a_{k_{l}-1}\right)}{k_{1} ! \cdots k_{n} !} \frac{d_{j-l}}{(j-l) !}
\end{aligned}
$$

Let

$$
F(x)=\sum_{n=1}^{\infty} c_{0}^{n+1}(n+1)^{2} x^{n} .
$$

Multiplying (6.15) by $x^{j}$ and then adding up from $j=1$ to $p$, we obtain

$$
\begin{aligned}
\sum_{j=1}^{p}\left(\frac{1}{4 M^{2}} \frac{a_{j}}{j !}+\frac{b_{j}}{j !}\right) x^{j} \\
\leq c_{0}^{\prime} a_{0} x+c_{0}^{\prime} x \sum_{j=1}^{p-1} \frac{a_{j}}{j !} x^{j}+c_{0} \sum_{j=1}^{p} \frac{d_{j}}{j !} x^{j} \\
+\left(\sum_{k=1}^{p-1}\left(\frac{a_{k}}{k !}+\frac{b_{k}}{k !}+v \frac{a_{k-1}}{(k-1) !}\right) x^{k}+\sum_{k=1}^{p} \frac{d_{k}}{k !} x^{k}\right) \\
\cdot F\left(\sum_{k=1}^{p-1}\left(\frac{a_{k}}{k !}+\frac{b_{k}}{k !}+v \frac{a_{k-1}}{(k-1) !}\right) x^{k}\right),
\end{aligned}
$$

where $c_{0}^{\prime}$ and $c_{0}$ depend on $\left\|z_{\alpha}\right\|_{H^{1}(3 Q \times \mathcal{T})},\left\|z_{\alpha \alpha}\right\|_{H^{1}(3 Q \times \mathcal{T})},\left\|z_{\alpha \alpha \alpha}\right\|_{H^{1}(3 Q \times \mathcal{T})},|Q|$, $|\mathcal{T}|, m, M$, and $\rho$. It is clear that if the radius of convergence of the series $\sum_{j=1}^{\infty} \frac{d_{j}}{j !} x^{j}$ is nonzero, then from an induction argument we have that the series $\sum_{j=1}^{\infty}\left(\frac{a_{j}}{j !}+\frac{b_{j}}{j !}\right) x^{j}$ has a nonzero radius of convergence. This in turn gives the analyticity of $z_{\alpha}(\cdot, t)$ on the interval $(a, b)$ for each fixed $t \in(0, T)$. We now construct a function $\eta$ for which the series $\sum_{j=1}^{\infty} \frac{d_{j}}{j !} x^{j}$ is convergent for some $x=r_{0}>0$.

\subsection{The Construction of $\boldsymbol{\eta}$}

We construct an $\eta$ such that

$$
\sum_{j=1}^{\infty} \frac{\left\|\partial_{\alpha}^{2}\left(\partial_{\alpha} \eta\right)^{j} 1\right\|_{L^{2}(\mathbb{R})}}{j !} r_{0}^{j}<\infty
$$

for some $r_{0}>0$. Moreover, this function $\eta$ satisfies that $\eta \in C^{\infty}(Q) \cap C_{0}^{3}(\mathbb{R})$, supp $\eta \subset Q$, and $\eta(\alpha) \geq 0$ for $\alpha \in \mathbb{R}$. 
For any one-variable functions $f$ and $g$, we denote $f \circ g(x)=f(g(x))$. From the chain rule, we know for any differentiable functions $f$ and $g, g$ strictly increasing, $(f \circ g)^{\prime}(x)=g^{\prime}(x) f^{\prime} \circ g(x)=\left(g^{\prime} \circ g^{-1} f^{\prime}\right) \circ g(x)$ and $(f \circ g)^{(j)}(x)=$ $\left(g^{\prime} \circ g^{-1} \partial_{x}\right)^{j} f \circ g(x)$ for $j \geq 1$.

Let

$$
g^{\prime}(\beta)=\left(1+\beta^{2}\right)^{-\gamma} \quad \text { for } \beta \in \mathbb{R}, \quad \frac{1}{2}<\gamma<\frac{3}{5},
$$

and $g(\beta)=\int_{-\infty}^{\beta} g^{\prime}(x) d x$ for $\beta \in \mathbb{R}$. We know $g: \mathbb{R} \rightarrow(0, q)$ is a homeomorphism, where $q=\int_{-\infty}^{\infty}\left(1+\beta^{2}\right)^{-\gamma} d \beta<\infty$. We define $\eta$ by

$$
\eta(\alpha)= \begin{cases}g^{\prime} \circ g^{-1}(\alpha)=\frac{1}{\left(g^{-1}\right)^{\prime}(\alpha)} & \text { for } \alpha \in(0, q) \\ 0 & \text { for } \alpha \notin(0, q)\end{cases}
$$

where $g$ is as given in (6.18). We claim that the function $\eta$, defined by (6.19) and (6.18), satisfies all the properties required for $Q=[0, q]$. For a general interval $Q$, we can modify the definition by some rescaling and translation.

The main properties that need to be checked are $\eta \in C^{3}(\mathbb{R})$ and (6.17). We may verify that $\eta \in C^{3}(\mathbb{R})$ by the fact $\eta \circ g(\beta)=g^{\prime}(\beta)$ for $\beta \in \mathbb{R}$ and application of the chain rule. We leave the details to the reader. We note that $\left(\eta \partial_{\alpha}\right)^{j} \eta \circ g=(\eta \circ g)^{(j)}$. A direct calculation gives that

$$
\begin{aligned}
\partial_{\alpha}^{2}\left(\partial_{\alpha} \eta\right)^{j} 1=\left(-\frac{\eta^{\prime \prime}}{\eta^{2}}+2 \frac{\eta^{\prime 2}}{\eta^{3}}\right)\left(\eta \partial_{\alpha}\right)^{j} \eta-3 \frac{\eta^{\prime}}{\eta^{3}}\left(\eta \partial_{\alpha}\right)^{j+1} \eta+\frac{1}{\eta^{3}}\left(\eta \partial_{\alpha}\right)^{j+2} \eta & \text { on }(0, q) .
\end{aligned}
$$

Therefore

$$
\left\|\partial_{\alpha}^{2}\left(\partial_{\alpha} \eta\right)^{j} 1\right\|_{L^{2}(\mathbb{R})}^{2} \leq c \sum_{\iota=0}^{2} \int\left(1+\beta^{2}\right)^{1+\iota}\left|(\eta \circ g)^{(j+\iota)}(\beta)\right|^{2} d \beta
$$

for some constant $c>0$. Estimate (6.20) is obtained from a change of variable $\alpha=g(\beta)$ in the integral. We may verify that (6.17) holds from estimating the righthand side integral in (6.20); this can be done either directly or by using Fourier analysis. We note that the Fourier transform of $\eta \circ g=g^{\prime}=\left(1+\beta^{2}\right)^{-\gamma}$ can be found in Stein [46, chap. V, sec. 3, p. 132]. We leave the precise verification of (6.17) to the reader.

\subsection{Analytic Compatibility of the Initial Data}

Let $w=\operatorname{Im}\left((1+i) \ln z_{\alpha}(\cdot, 0)\right)$ and $v=\operatorname{Re}\left((1+i) \ln z_{\alpha}(\cdot, 0)\right)$, and assume in addition that $w$ is analytic on $(a, b)$. We now show that $v$ is also analytic on $(a, b)$. So far we know under this further assumption that we have $v \in C^{\infty}(a, b)$, $z_{\alpha}(\cdot, 0) \in C^{\infty}(a, b)$, and $\partial_{\alpha}^{j} z_{\alpha} \in H_{\mathrm{loc}}^{1}((a, b) \times[0, T))$.

Let $Q, \eta$, and $\rho$ be defined as at the beginning of Section 6, and by (6.19) and (6.18), up to some rescaling and translation. Take $\mathcal{T}=\left[0, T_{1}\right] \subset[0, T)$. Let 
$\varsigma(t)=T_{1}-t$ for $t \in \mathcal{T}, \varsigma(t)=0$ for $t \notin \mathcal{T}$. We note that $\varsigma(0)=T_{1}=|\mathcal{T}|$. Define

$$
\begin{aligned}
\tilde{a}_{k} & =\left\|\varsigma^{k} \partial_{\alpha}^{3}\left(\eta \partial_{\alpha}\right)^{k} z\right\|_{L^{2}(3 Q \times \mathcal{T})}, \\
\tilde{b}_{k} & =\left\|\varsigma^{k} \partial_{\alpha}^{2}\left(\eta \partial_{\alpha}\right)^{k} z_{t}\right\|_{L^{2}(3 Q \times \mathcal{T})}, \\
\mu_{k} & =|\mathcal{T}|^{k}\left\||D|^{1 / 2} \partial_{\alpha}\left(\eta \partial_{\alpha}\right)^{k} \ln z_{\alpha}(\cdot, 0)\right\|_{L^{2}(\mathbb{R})}, \\
v_{k} & =|\mathcal{T}|^{k}\left\||D|^{1 / 2} \partial_{\alpha}\left(\partial_{\alpha} \eta\right)^{k} 1\right\|_{L^{2}(\mathbb{R})},
\end{aligned}
$$

for $k \geq 1$ and $\tilde{a}_{0}=\left\|\partial_{\alpha}^{2}\left(\eta z_{\alpha}\right)\right\|_{L^{2}(3 Q \times \mathcal{T})}, \mu_{0}=\left\||D|^{1 / 2}\left(\eta \partial_{\alpha}\right) \ln z_{\alpha}(\cdot, 0)\right\|_{L^{2}(\mathbb{R})}$.

We prove the analytic compatibility of the initial data by basically following the same procedure as in the proof of $H^{j+1 / 2}$ compatibility in Section 5. Before we proceed, we record the following identities:

LEMMA 6.1 We have

$$
\begin{aligned}
\partial_{\alpha}^{2}\left(\eta \partial_{\alpha}\right)^{j} z= & \sum_{l=0}^{j}\left(\begin{array}{l}
l \\
j
\end{array}\right) \partial_{\alpha}\left(\partial_{\alpha} \eta\right)^{j-l} 1\left(\eta \partial_{\alpha}\right)^{l} z_{\alpha} \\
& +\sum_{l=0}^{j}\left(\begin{array}{l}
l \\
j
\end{array}\right)\left(\partial_{\alpha} \eta\right)^{j-l} 1 \partial_{\alpha}\left(\eta \partial_{\alpha}\right)^{l} z_{\alpha}
\end{aligned}
$$

and

$$
\frac{1}{l !}\left(\eta \partial_{\alpha}\right)^{l} z_{\alpha}=z_{\alpha} \sum_{n=1}^{l} \frac{1}{n !} \sum_{\substack{k_{1}+\ldots+k_{n}=l \\ k_{l} \geq 1}} \frac{\prod_{l=1}^{n}\left(\eta \partial_{\alpha}\right)^{k_{l}} \ln z_{\alpha}}{k_{1} ! \cdots k_{n} !} .
$$

The proof of (6.22) is straightforward, and we leave it to the reader. Equality (6.23) is inspired by

$$
\frac{1}{l !}\left(e^{f}\right)^{(l)}(0)=e^{f(0)} \sum_{n=1}^{l} \frac{1}{n !} \sum_{\substack{k_{1}+\ldots+k_{n}=l \\ k_{l} \geq 1}} \frac{f^{\left(k_{1}\right)}(0) \cdots f^{\left(k_{n}\right)}(0)}{k_{1} ! \cdots k_{n} !}
$$

which in turn is obtained from Taylor expansion. It can also be proved directly by induction.

From (6.22) and (6.23), we obtain

$$
\frac{\partial_{\alpha}^{2}\left(\eta(\alpha) \partial_{\alpha}\right)^{j} z(\alpha, 0)}{z_{\alpha}(\alpha, 0)}=\partial_{\alpha}\left(\eta(\alpha) \partial_{\alpha}\right)^{j} \ln z_{\alpha}(\alpha, 0)+j ! \mathcal{R}(\alpha, 0)
$$


where

$$
\begin{aligned}
\mathcal{R}= & \sum_{l=0}^{j-1} \frac{\partial_{\alpha}\left(\partial_{\alpha} \eta\right)^{j-l} 1}{(j-l) !} \frac{\left(\eta \partial_{\alpha}\right)^{l} z_{\alpha}}{l ! z_{\alpha}}+\sum_{l=0}^{j-1} \frac{\left(\partial_{\alpha} \eta\right)^{j-l} 1}{(j-l) !} \frac{\partial_{\alpha}\left(\eta \partial_{\alpha}\right)^{l} z_{\alpha}}{l ! z_{\alpha}} \\
& +\partial_{\alpha}\left(\ln z_{\alpha}\right) \sum_{n=1}^{j} \frac{1}{n !} \sum_{\substack{k_{1}+\cdots+k_{n}=j \\
k_{l} \geq 1}} \frac{\prod_{l=1}^{n}\left(\eta \partial_{\alpha}\right)^{k_{l}} \ln z_{\alpha}}{k_{1} ! \cdots k_{n} !} \\
& +\sum_{n=2}^{j} \frac{1}{n !} \sum_{\substack{k_{1}+\cdots+k_{n}=j \\
k_{l} \geq 1}} \frac{\partial_{\alpha}\left(\prod_{l=1}^{n}\left(\eta \partial_{\alpha}\right)^{k_{l}} \ln z_{\alpha}\right)}{k_{1} ! \cdots k_{n} !} .
\end{aligned}
$$

Further expanding the terms

$$
\frac{\left(\eta \partial_{\alpha}\right)^{l} z_{\alpha}}{z_{\alpha}}, \quad \frac{\partial_{\alpha}\left(\eta \partial_{\alpha}\right)^{l} z_{\alpha}}{z_{\alpha}}, \quad \text { and } \quad \partial_{\alpha}\left(\prod_{\iota=1}^{n}\left(\eta \partial_{\alpha}\right)^{k_{l}} \ln z_{\alpha}\right)
$$

in $\mathcal{R}$ and applying Lemma 5.9, we obtain the following estimate for $\mathcal{R}(\alpha, 0)$ :

$$
\begin{aligned}
& |\mathcal{T}|^{j}\left\||D|^{1 / 2} \mathcal{R}(\cdot, 0)\right\|_{L^{2}(\mathbb{R})} \\
& \leq \sum_{n=2}^{j} \frac{n \kappa_{0}^{n}}{n !} \sum_{\substack{k_{1}+\ldots+k_{n}=j \\
k_{l} \geq 1}} \frac{\mu_{k_{1}} \cdots \mu_{k_{n}}}{k_{1} ! \cdots k_{n} !}+\kappa_{0} \frac{1}{j !} \mu_{j-1} \\
& +\sum_{l=1}^{j-1} \sum_{n=1}^{l} \frac{(n+1) \kappa_{0}^{n+1}}{n !} \sum_{\substack{k_{1}+\ldots+k_{n}=l \\
k_{l} \geq 1}} \frac{\mu_{k_{1}} \cdots \mu_{k_{n}}}{k_{1} ! \cdots k_{n} !} \frac{v_{j-l}}{(j-l) !}+\kappa_{0} \frac{1}{j !} v_{j}
\end{aligned}
$$

where $\kappa_{0}$ is a constant depending on $|Q|$ and $\left\|\ln z_{\alpha}(\cdot, 0)\right\|_{H^{5 / 2}(3 Q)}$.

We are now ready to prove the analytic compatibility of the initial data. Working through a similar argument to that in Section 5 and using (6.24), we arrive at

$$
\begin{aligned}
& \iint \varsigma^{2 j}(t)\left|\partial_{\alpha}^{2}\left(\eta \partial_{\alpha}\right)^{j} z_{t}(\alpha, t)\right|^{2} d \alpha d t \\
& \quad+\frac{1}{8 M^{4}} \iint \varsigma^{2 j}(t)\left|\partial_{\alpha}^{3}\left(\eta \partial_{\alpha}\right)^{j} z(\alpha, t)\right|^{2} d \alpha d t \\
& \quad+\left.\left.\frac{|\mathcal{T}|^{2 j}}{4} \int|| D\right|^{1 / 2}\left(\partial_{\alpha}\left(\eta \partial_{\alpha}\right)^{j} \ln z_{\alpha}(\alpha, 0)\right)\right|^{2} d \alpha
\end{aligned}
$$




$$
\begin{aligned}
\leq & c_{5} j^{2} \iint \varsigma(t)^{2(j-1)}\left|\partial_{\alpha}^{3}\left(\eta \partial_{\alpha}\right)^{j-1} z(\alpha, t)\right|^{2} d \alpha d t \\
& +\left.\left.\frac{|\mathcal{T}|^{2 j}}{2} \int|| D\right|^{1 / 2}\left(\partial_{\alpha}\left(\eta \partial_{\alpha}\right)^{j} w(\alpha)\right)\right|^{2} d \alpha \\
& +\left.\left.\frac{3|\mathcal{T}|^{2 j}}{2} j !^{2} \int|| D\right|^{1 / 2} \mathcal{R}(\alpha, 0)\right|^{2} d \alpha+\iint \varsigma^{2 j}(t)|\mathcal{M}|^{2} d \alpha d t,
\end{aligned}
$$

where the constant $c_{5}$ depends on $\left\|z_{\alpha}\right\|_{H^{1}(3 Q \times \mathcal{T})},\left\|z_{\alpha \alpha}\right\|_{H^{1}(3 Q \times \mathcal{T})},\left\|z_{\alpha \alpha \alpha}\right\|_{H^{1}(3 Q \times \mathcal{T})}$, $|Q|,|\mathcal{T}|, m, M$, and $\rho$. Here the terms involving the initial data are as usual coming from the fact that $\varsigma(0)=|\mathcal{T}| \neq 0$ and from integration by parts.

Notice that

$$
\mathcal{M}=\frac{j !}{2 \pi i}\left(-E-\mathcal{D}+\left(\rho(\alpha) \partial_{\alpha}\right)^{2} A+\left(\rho(\alpha) \partial_{\alpha}\right)^{2} B\right),
$$

while the terms $\left\|\zeta^{j}\left(\rho \partial_{\alpha}\right)^{2} A\right\|_{L^{2}(R \times \mathcal{T})},\left\|\zeta^{j}\left(\rho \partial_{\alpha}\right)^{2} B\right\|_{L^{2}(R \times \mathcal{T})},\left\|\zeta^{j} E\right\|_{L^{2}(R \times \mathcal{T})}$, and $\left\|S^{j} \mathcal{D}\right\|_{L^{2}(R \times \mathcal{T})}$ can be estimated very much the same as in (6.6), (6.7), (6.8), and (6.11). We obtain, from (6.26), that

$$
\begin{aligned}
& \frac{1}{j !}\left(\frac{1}{4 M^{2}} \tilde{a}_{j}+\tilde{b}_{j}+\frac{1}{2} \mu_{j}\right) \\
& \quad \leq 2|\mathcal{T}|^{j} \frac{\left\||D|^{1 / 2} \partial_{\alpha}\left(\eta \partial_{\alpha}\right)^{j} w\right\|_{L^{2}(\mathbb{R})}}{j !}+3 \kappa_{0}\left(\frac{1}{j !} \mu_{j-1}+\frac{1}{j !} v_{j}\right) \\
& \quad+3 \sum_{n=2}^{j} \frac{n \kappa_{0}^{n}}{n !} \sum_{\substack{k_{1}+\cdots+k_{n}=j \\
k_{l} \geq 1}} \frac{\mu_{k_{1}} \cdots \mu_{k_{n}}}{k_{1} ! \cdots k_{n} !} \\
& \quad+3 \sum_{l=1}^{j-1} \sum_{n=1}^{l} \frac{(n+1) \kappa_{0}^{n+1}}{n !} \sum_{k_{1}+\cdots+k_{n}=l} \frac{\mu_{k_{l}} \cdots \mu_{k_{n}}}{k_{1} ! \cdots k_{n} !} \frac{v_{j-l}}{(j-l) !}+\tilde{c}_{0} \frac{1}{(j-1) !} \tilde{a}_{j-1} \\
& \quad+\tilde{c}_{0} \frac{d_{j}}{j !}+\sum_{n=2}^{j} \sum_{k_{1}+\cdots+k_{n}=j} \tilde{c}_{k_{l} \geq 1}^{n} n^{2} \frac{\prod_{l=1}^{n}\left(\tilde{a}_{k_{l}}+\tilde{b}_{k_{l}}+\tilde{v} k_{l} \tilde{a}_{k_{l}-1}\right)}{k_{1} ! \cdots k_{n} !} \\
& \quad+\sum_{l=1}^{j-1} \sum_{n=1}^{l} \sum_{k_{1}+\ldots+k_{n}=l} \tilde{c}_{k_{l} \geq 1}^{n+1}(n+1)^{2} \frac{\prod_{l=1}^{n}\left(\tilde{a}_{k_{l}}+\tilde{b}_{k_{l}}+\tilde{v} k_{l} \tilde{a}_{k_{l}-1}\right)}{k_{1} ! \cdots k_{n} !} \frac{d_{j-l}}{(j-l) !}
\end{aligned}
$$

where the constant $\tilde{c}_{0}$ depends on $\left\|z_{\alpha}\right\|_{H^{1}(3 Q \times \mathcal{T})},\left\|z_{\alpha \alpha}\right\|_{H^{1}(3 Q \times \mathcal{T})},\left\|z_{\alpha \alpha \alpha}\right\|_{H^{1}(3 Q \times \mathcal{T})}$, $|Q|,|\mathcal{T}|, m, M$, and $\rho$. From our assumption and the construction of $\eta$, we know there exists $r_{0}>0$ such that

$$
\begin{aligned}
& \sum_{j=1}^{\infty}|\mathcal{T}|^{j} \frac{\left\||D|^{1 / 2} \partial_{\alpha}\left(\eta \partial_{\alpha}\right)^{j} w\right\|_{L^{2}(\mathbb{R})}}{j !} r_{0}^{j}<\infty \\
& \sum_{j=1}^{\infty} \frac{v_{j}}{j !} r_{0}^{j}<\infty, \quad \text { and } \quad \sum_{j=1}^{\infty} \frac{d_{j}}{j !} r_{0}^{j}<\infty
\end{aligned}
$$


Multiplying (6.27) by $x^{j}$ and adding up from $j=1$ to $p$, we then conclude from an inductive argument that the series

$$
\sum_{j=1}^{\infty} \frac{1}{j !}\left(\frac{1}{4 M^{2}} \tilde{a}_{j}+\tilde{b}_{j}+\frac{1}{2} \mu_{j}\right) x^{j}
$$

has a nonzero radius of convergence. The analyticity of $\ln z_{\alpha}(\cdot, 0)$ on $(a, b)$ therefore follows.

\section{Existence}

We now prove Theorem 1.4. That is, we show that for any given real-valued function $w_{0} \in H^{3 / 2}(\mathbb{R})$, there exists a $T=T\left(\left\|w_{0}\right\|_{H^{3 / 2}}\right)>0$ such that the Birkhoff-Rott equation (1.6) has a solution $z=z(\alpha, t)$ for $0 \leq t \leq T$ satisfying $\ln z_{\alpha} \in C\left([0, T], H^{3 / 2}(\mathbb{R})\right) \cap C^{1}\left([0, T], H^{1 / 2}(\mathbb{R})\right)$ and $\operatorname{Im}\left((1+i) \ln z_{\alpha}(\alpha, 0)=\right.$ $w_{0}(\alpha)$. Moreover, this solution satisfies

$$
m|\alpha-\beta| \leq|z(\alpha, t)-z(\beta, t)| \leq M|\alpha-\beta| \text { for } \alpha, \beta \in \mathbb{R}, 0 \leq t \leq T,
$$

for some constants $m, M>0$.

We first rewrite equation (1.6). Taking the derivative with respect to $\alpha$ on both sides of (1.6) and then dividing both sides by $\bar{z}_{\alpha}$, we get

$$
\begin{aligned}
\overline{\partial_{t}\left(\ln z_{\alpha}\right)}= & -\frac{1}{2 i\left|z_{\alpha}\right|^{2}}|D| \ln z_{\alpha}+\frac{1}{2 i\left|z_{\alpha}\right|^{2}}|D| \ln z_{\alpha} \\
& +\frac{1}{2 \pi i \bar{z}_{\alpha}(\alpha, t)} \int \frac{-z_{\alpha}(\alpha, t)+z_{\beta}(\beta, t)}{(z(\alpha, t)-z(\beta, t))^{2}} d \beta .
\end{aligned}
$$

Let

$$
\begin{gathered}
V=(1+i) \ln z_{\alpha}, \quad v=\operatorname{Re} V, w=\operatorname{Im} V, \\
\mathfrak{a}=\frac{1}{2\left|z_{\alpha}\right|^{2}}=\frac{1}{2} e^{-(v+w)}, \\
\mathcal{F}(\alpha, t)=\frac{1-i}{2 \pi i}\left(\frac{\pi}{\left|z_{\alpha}(\alpha, t)\right|^{2}}|D| \ln z_{\alpha}(\alpha, t)\right. \\
\left.+\frac{1}{\bar{z}_{\alpha}(\alpha, t)} \int \frac{-z_{\alpha}(\alpha, t)+z_{\beta}(\beta, t)}{(z(\alpha, t)-z(\beta, t))^{2}} d \beta\right) \\
\mathfrak{f}_{1}(\alpha, t)=\operatorname{Re} \mathcal{F}(\alpha, t), \quad \mathfrak{f}_{2}(\alpha, t)=\operatorname{Im} \mathcal{F}(\alpha, t) .
\end{gathered}
$$

Equation (7.2) becomes

$$
\bar{V}_{t}=\mathfrak{a}|D| V+\mathcal{F}
$$

or equivalently

$$
\left\{\begin{array}{l}
v_{t}-\mathfrak{a}|D| v=\mathfrak{f}_{1} \\
w_{t}+\mathfrak{a}|D| w=-\mathfrak{f}_{2}
\end{array}\right.
$$


Our goal is to solve (7.5)-(7.3) for some finite time period $[0, T]$ for any given $w(\alpha, 0)=w_{0}(\alpha) \in H^{3 / 2}(\mathbb{R})$. Let's first record some basic estimates.

LEMMA 7.1

(i) For any $f \in H^{1 / 4}(\mathbb{R})$,

$$
\|f\|_{L^{4}(\mathbb{R})} \leq c\left\||D|^{1 / 4} f\right\|_{L^{2}(\mathbb{R})} .
$$

(ii) For any $f \in H^{1 / 2}(\mathbb{R})$,

$$
\|f\|_{\mathrm{BMO}(\mathbb{R})} \leq \sqrt{2 \pi}\left\||D|^{1 / 2} f\right\|_{L^{2}(\mathbb{R})} .
$$

(iii) Let $f \in H^{1}(\mathbb{R})$ and $g \in H^{1 / 2}(\mathbb{R})$. Then $f g \in H^{1 / 2}(\mathbb{R})$, and

$$
\|f g\|_{H^{1 / 2}(\mathbb{R})} \leq c\|f\|_{H^{1}(\mathbb{R})}\|g\|_{H^{1 / 2}(\mathbb{R})} .
$$

Here $c$ is a universal constant.

PROOF:

(i) This proof can be found in Stein [46, chap. V, sec. 1].

(ii) This statement is a direct consequence of definitions:

$$
\begin{aligned}
\|f\|_{\mathrm{BMO}(\mathbb{R})} & \leq \sup _{I}\left(\frac{1}{|I|^{2}} \iint_{I \times I}|f(\alpha)-f(\beta)|^{2} d \alpha d \beta\right)^{1 / 2} \\
& \leq\left(\iint \frac{|f(\alpha)-f(\beta)|^{2}}{(\alpha-\beta)^{2}} d \alpha d \beta\right)^{1 / 2}
\end{aligned}
$$

(iii) The proof of (iii) is also straightforward: notice that

$$
\|f g\|_{L^{2}(\mathbb{R})} \leq\|f\|_{L^{\infty}(\mathbb{R})}\|g\|_{L^{2}(\mathbb{R})}
$$

and

$$
\begin{aligned}
& \left(\iint \frac{|f(\alpha) g(\alpha)-f(\beta) g(\beta)|^{2}}{(\alpha-\beta)^{2}} d \alpha d \beta\right)^{1 / 2} \\
& \leq\left(\iint \frac{|f(\alpha)-f(\beta)|^{2}|g(\alpha)|^{2}}{(\alpha-\beta)^{2}} d \alpha d \beta\right)^{1 / 2} \\
& \quad+\|f\|_{L^{\infty}(\mathbb{R})}\left(\iint \frac{|g(\alpha)-g(\beta)|^{2}}{(\alpha-\beta)^{2}} d \alpha d \beta\right)^{1 / 2} \\
& \leq \sqrt{2 \pi}\left(\int|g(\alpha)|^{2}\left|[f, H] f_{\alpha}(\alpha)\right| d \alpha\right)^{1 / 2}+\sqrt{2 \pi}\|f\|_{L^{\infty}}\left\||D|^{1 / 2} g\right\|_{L^{2}(\mathbb{R})} \\
& \leq c\|g\|_{L^{4}(\mathbb{R})}\left(\|f\|_{\text {BMO }(\mathbb{R})}\left\|f_{\alpha}\right\|_{L^{2}(\mathbb{R})}\right)^{1 / 2}+c\|f\|_{H^{1}(\mathbb{R})}\left\||D|^{1 / 2} g\right\|_{L^{2}(\mathbb{R})} .
\end{aligned}
$$

A further application of (i) and (ii) gives (iii). 
We present and prove the result in Lemma 7.2(ii). A much stronger and deeper result on the analytic dependence of the Cauchy integral in $\mathrm{BMO}(\mathbb{R})$ is available in $[8,12,13]$ (see also Theorem 2.3 and Corollary 2.4). Lemma 7.2(i) and its proof are modifications of similar results in [15].

Let

$$
C_{z} f(\alpha)=\int \frac{z_{\alpha}(\alpha)}{z(\alpha)-z(\beta)} f(\beta) d \beta
$$

LEMMA 7.2

(i) Let $z^{1}=z^{1}(\alpha)$ be chord-arc,

$$
m|\alpha-\beta| \leq\left|z^{1}(\alpha)-z^{1}(\beta)\right| \leq M|\alpha-\beta|, \quad \alpha, \beta \in \mathbb{R} .
$$

for some constants $m, M>0$. Let $b=b(\alpha)$ be a complex-valued function in $\operatorname{BMO}(\mathbb{R})$ such that $\operatorname{Re} b \in L^{\infty}, \tilde{M}^{-1} \leq e^{\operatorname{Re} b(\alpha)} \leq \tilde{M}$ for some constant $\tilde{M}$, and $\|b\|_{\mathrm{BMO}(\mathbb{R})} \leq m /\left(2 M \tilde{M}^{2}\right)$. Then $z_{\alpha}=z_{\alpha}^{1} e^{b}$ defines a chord-arc curve $z=z(\alpha)$, with

$$
\frac{m}{2 \tilde{M}}|\alpha-\beta| \leq|z(\alpha)-z(\beta)| \leq\left(\frac{m}{2 \tilde{M}}+M \tilde{M}\right)|\alpha-\beta| \quad \text { for } \alpha, \beta \in \mathbb{R} .
$$

(ii) Let $z^{1}=z^{1}(\alpha)$ and $z^{2}=z^{2}(\alpha)$ be given, and $\ln z_{\alpha}(\alpha, \theta)=\ln z_{\alpha}^{1}(\alpha)+$ $\theta\left(\ln z_{\alpha}{ }^{2}(\alpha)-\ln z_{\alpha}{ }^{1}(\alpha)\right)$ for $0 \leq \theta \leq 1$. Assume that there exist constants $m, M>0$ such that

$$
m|\alpha-\beta| \leq|z(\alpha, \theta)-z(\beta, \theta)| \leq M|\alpha-\beta| \quad \text { for } \alpha, \beta \in \mathbb{R}, 0 \leq \theta \leq 1 .
$$

Then there exists a constant $c$ depending on $m$ and $M$ such that

$$
\begin{aligned}
& \left\|\left(C_{z^{1}}-C_{z^{2}}\right) f\right\|_{L^{2}(\mathbb{R})} \leq c\left\||D|^{1 / 2}\left(\ln z_{\alpha}{ }^{1}-\ln z_{\alpha^{2}}{ }^{2}\right)\right\|_{L^{2}(\mathbb{R})}\|f\|_{L^{2}(\mathbb{R})} \\
& \text { for all } f \in L^{2}(\mathbb{R}) .
\end{aligned}
$$

ProOF:

(i) Let $m_{I} b=\frac{1}{|I|} \int_{I} b(\alpha) d \alpha$ for any interval $I$. We know

$$
\begin{aligned}
& \left|z(\alpha)-z(\beta)-e^{m_{[\alpha, \beta]} b}\left(z^{1}(\alpha)-z^{1}(\beta)\right)\right| \\
& =\left|\int_{\beta}^{\alpha} z_{\gamma}^{1}(\gamma)\left(e^{b(\gamma)}-e^{m_{[\alpha, \beta]} b}\right) d \gamma\right| \leq M \tilde{M}\left|\int_{\beta}^{\alpha}\right| b(\gamma)-m_{[\alpha, \beta]} b|d \gamma| \\
& \leq M \tilde{M}\left\|b_{0}\right\|_{\mathrm{BMO}}|\alpha-\beta| .
\end{aligned}
$$

On the other hand, $m \tilde{M}^{-1}|\alpha-\beta| \leq\left|e^{m_{[\alpha, \beta]} b}\left(z^{1}(\alpha)-z^{1}(\beta)\right)\right| \leq M \tilde{M}|\alpha-\beta|$. This proves (7.7). 
(ii) We know from assumption that $z_{\alpha}(\alpha, 0)=z_{\alpha}^{1}(\alpha)$ and $z_{\alpha}(\alpha, 1)=z_{\alpha}^{2}(\alpha)$. Now

$$
\begin{array}{rl}
C_{z^{1}} & f(\alpha)-C_{z^{2}} f(\alpha) \\
& =-\int_{0}^{1} \int \partial_{\theta} \frac{z_{\alpha}(\alpha, \theta)}{z(\alpha, \theta)-z(\beta, \theta)} f(\beta) d \beta d \theta \\
& =\int_{0}^{1} \int \frac{z_{\alpha}(\alpha, \theta) \int_{\beta}^{\alpha} z_{\gamma}(\gamma, \theta)\left(\ln z_{\gamma}^{2}(\gamma)-\ln z_{\gamma}^{1}(\gamma)-\ln z_{\alpha}^{2}(\alpha)+\ln z_{\alpha}^{1}(\alpha)\right) d \gamma}{(z(\alpha, \theta)-z(\beta, \theta))^{2}} f(\beta) d \beta d \theta .
\end{array}
$$

Therefore

$$
\begin{aligned}
& \left|C_{z^{1}} f(\alpha)-C_{z^{2}} f(\alpha)\right|^{2} \\
\leq & c\left(\int\left|\frac{\ln z_{\alpha}^{2}(\alpha)-\ln z_{\alpha}^{1}(\alpha)-\ln z_{\beta}^{2}(\beta)+\ln z_{\beta}^{1}(\beta)}{\alpha-\beta}\right|^{2} d \beta\right)\left(\int|f(\beta)|^{2} d \beta\right)
\end{aligned}
$$

for some constant $c$ depending on $m$ and $M$. Estimate (7.8) follows from integrating with respect to $\alpha$ on both sides of (7.9).

Lemma 7.3 Let $T>0, \mathfrak{a}$, and $\mathcal{F}$ be defined by (7.3). Assume that $V \in C([0, T]$, $\left.H^{3 / 2}(\mathbb{R})\right)$ and there exist constants $m, M>0$ such that

$$
m|\alpha-\beta| \leq|z(\alpha, t)-z(\beta, t)| \leq M|\alpha-\beta| \quad \text { for } \alpha, \beta \in \mathbb{R}, t \in[0, T] .
$$

Then $\mathfrak{a}-1 \in C\left([0, T], H^{3 / 2}(\mathbb{R})\right)$ and $\mathcal{F} \in C\left([0, T], H^{1 / 2}(\mathbb{R})\right)$, and there exists a constant $c$ depending on $m$ and $M$ only such that for any $t \in[0, T]$,

$$
\begin{aligned}
\|\mathfrak{a}(\cdot, t)-1\|_{H^{3 / 2}(\mathbb{R})} & \leq c\left(1+\|V(\cdot, t)\|_{H^{1}(\mathbb{R})}\right)\|V(\cdot, t)\|_{H^{3 / 2}(\mathbb{R})}, \\
\|\mathcal{F}(\cdot, t)\|_{L^{2}(\mathbb{R})} & \leq c\left\|\partial_{\alpha} V(\cdot, t)\right\|_{L^{2}(\mathbb{R})}, \\
\|\mathcal{F}(\cdot, t)\|_{H^{1 / 2}(\mathbb{R})} & \leq c\left(1+\|V(\cdot, t)\|_{H^{1}}\right)^{3}\|V(\cdot, t)\|_{H^{3 / 2}(\mathbb{R})} .
\end{aligned}
$$

If in addition $V \in L^{2}\left([0, T], H^{2}(\mathbb{R})\right)$, then $\partial_{\alpha} \mathcal{F} \in L^{2}(\mathbb{R} \times[0, T])$, and there exists a constant $c$ depending only on $m$ and $M$ such that for a.e. $t \in[0, T]$ and any $\varepsilon>0$,

$$
\begin{aligned}
\left\|\partial_{\alpha} \mathcal{F}(\cdot, t)\right\|_{L^{2}(\mathbb{R})}^{2} \leq & c\left(1+\frac{1}{\varepsilon}\left\||D|^{1 / 2} V(\cdot, t)\right\|_{L^{2}(\mathbb{R})}^{2}\right)\|V(\cdot, t)\|_{H^{3 / 2}(\mathbb{R})}^{4} \\
& +\varepsilon\left\|V_{\alpha \alpha}(\cdot, t)\right\|_{L^{2}(\mathbb{R})}^{2} .
\end{aligned}
$$

Proof: That $\mathfrak{a}-1 \in C\left([0, T], H^{3 / 2}(\mathbb{R})\right)$ and (7.10) directly follow from Lemma 7.1. The first estimate in (7.11) is straightforward. Notice that from integration by parts,

$$
\int \frac{-z_{\alpha}(\alpha, t)+z_{\beta}(\beta, t)}{(z(\alpha, t)-z(\beta, t))^{2}} d \beta=-\int \frac{z_{\alpha}(\alpha, t)}{z(\alpha, t)-z(\beta, t)} \frac{\partial_{\beta} \ln z_{\beta}(\beta, t)}{z_{\beta}(\beta, t)} d \beta
$$


Let $t \in[0, T]$ be fixed, and

$$
\tilde{C} f(\alpha, t)=\int \frac{1}{z(\alpha, t)-z(\beta, t)} f(\beta) d \beta .
$$

We know $\tilde{C}$ is bounded from $L^{2}(\mathbb{R}) \rightarrow L^{2}(\mathbb{R})$, with

$$
\|\tilde{C} f(\cdot, t)\|_{L^{2}(\mathbb{R})} \leq c\|f\|_{L^{2}(\mathbb{R})}
$$

for some constant $c$ depending on $m$ and $M$ (see Section 1, Theorem 1.1, [17, 5, $11,13])$. Moreover, we know from integration by parts that

$$
\partial_{\alpha} \tilde{C} f(\alpha)=\int \frac{z_{\alpha}(\alpha, t)}{z(\alpha, t)-z(\beta, t)} \partial_{\beta} \frac{f(\beta)}{z_{\beta}(\beta, t)} d \beta .
$$

Therefore $\tilde{C}$ is bounded from $H^{1}(\mathbb{R})$ to $H^{1}(\mathbb{R})$ with

$$
\|\tilde{C} f(\cdot, t)\|_{H^{1}(\mathbb{R})} \leq c\left(1+\|V(\cdot, t)\|_{H^{1}(\mathbb{R})}\right)\|f\|_{H^{1}(\mathbb{R})} ;
$$

here $c$ is a constant depending on $m$ and $M$. Using interpolation, we have $\tilde{C}$ : $H^{1 / 2}(\mathbb{R}) \rightarrow H^{1 / 2}(\mathbb{R})$ bounded and

$$
\|\tilde{C} f(\cdot, t)\|_{H^{1 / 2}(\mathbb{R})} \leq c\left(1+\|V(\cdot, t)\|_{H^{1}(\mathbb{R})}\right)\|f\|_{H^{1 / 2}(\mathbb{R})}
$$

for some constant $c$ depending on $m$ and $M$. The second estimate in (7.11) therefore follows directly from the definition of $\mathcal{F}$ and an application of Lemma 7.1(iii). The fact that $\mathcal{F} \in C\left([0, T], H^{1 / 2}(\mathbb{R})\right)$ can be proved similarly using Lemma 7.1 and interpolation; we omit the details.

We now prove (7.12). We know

$$
\begin{aligned}
\frac{2 \pi i}{1-i} \partial_{\alpha} \mathcal{F}= & \partial_{\alpha}\left(\frac{1}{\bar{z}_{\alpha}(\alpha, t)}\right) \int \frac{-z_{\alpha}(\alpha, t)+z_{\beta}(\beta, t)}{(z(\alpha, t)-z(\beta, t))^{2}} d \beta \\
& +\partial_{\alpha}\left(\frac{\pi}{\left|z_{\alpha}(\alpha, t)\right|^{2}}\right)|D| \ln z_{\alpha}(\alpha, t) \\
& +\frac{2}{\bar{z}_{\alpha}(\alpha, t)} \int \frac{\left(-z_{\alpha}(\alpha, t)+z_{\beta}(\beta, t)\right)^{2}}{(z(\alpha, t)-z(\beta, t))^{3}} d \beta \\
& +\frac{1}{\bar{z}_{\alpha}(\alpha, t)} \int \frac{-z_{\alpha \alpha}(\alpha, t)+z_{\beta \beta}(\beta, t)}{(z(\alpha, t)-z(\beta, t))^{2}} d \beta \\
& +\frac{1}{\left|z_{\alpha}(\alpha, t)\right|^{2}} \int \frac{\partial_{\alpha} \ln z_{\alpha}(\alpha, t)-\partial_{\beta} \ln z_{\beta}(\beta, t)}{(\alpha-\beta)^{2}} d \beta
\end{aligned}
$$

From integration by parts, we have

$$
\begin{aligned}
& 2 \int \frac{\left(-z_{\alpha}(\alpha, t)+z_{\beta}(\beta, t)\right)^{2}}{(z(\alpha, t)-z(\beta, t))^{3}} d \beta \\
& \quad=\int \frac{z_{\alpha}^{2}(\alpha, t)-z_{\beta}^{2}(\beta, t)}{(z(\alpha, t)-z(\beta, t))^{2}} \frac{1}{z_{\beta}(\beta, t)} \partial_{\beta} \ln z_{\beta}(\beta, t) d \beta
\end{aligned}
$$




$$
\begin{aligned}
= & \int \frac{z_{\alpha}^{2}(\alpha, t)-z_{\beta}^{2}(\beta, t)}{(z(\alpha, t)-z(\beta, t))^{2}} \frac{1}{z_{\beta}(\beta, t)}\left(\partial_{\beta} \ln z_{\beta}(\beta, t)-\partial_{\alpha} \ln z_{\alpha}(\alpha, t)\right) d \beta \\
& +\partial_{\alpha} \ln z_{\alpha}(\alpha, t) \int \frac{z_{\alpha}^{2}(\alpha, t)-z_{\beta}^{2}(\beta, t)}{(z(\alpha, t)-z(\beta, t))^{2}} \frac{1}{z_{\beta}(\beta, t)} d \beta .
\end{aligned}
$$

Furthermore,

$$
\begin{aligned}
& \int \frac{-z_{\alpha \alpha}(\alpha, t)+z_{\beta \beta}(\beta, t)}{(z(\alpha, t)-z(\beta, t))^{2}} d \beta \\
& +\frac{1}{z_{\alpha}(\alpha, t)} \int \frac{\partial_{\alpha} \ln z_{\alpha}(\alpha, t)-\partial_{\beta} \ln z_{\beta}(\beta, t)}{(\alpha-\beta)^{2}} d \beta \\
& =\int\left(\frac{-z_{\alpha} \partial_{\alpha} \ln z_{\alpha}(\alpha, t)+z_{\beta} \partial_{\beta} \ln z_{\beta}(\beta, t)}{(z(\alpha, t)-z(\beta, t))^{2}}\right. \\
& \left.\quad+\frac{1}{z_{\alpha}(\alpha, t)} \frac{\partial_{\alpha} \ln z_{\alpha}(\alpha, t)-\partial_{\beta} \ln z_{\beta}(\beta, t)}{(\alpha-\beta)^{2}}\right) d \beta \\
& \quad \partial_{\alpha} \ln z_{\alpha}(\alpha, t) \int \frac{-z_{\alpha}(\alpha, t)+z_{\beta}(\beta, t)}{(z(\alpha, t)-z(\beta, t))^{2}} d \beta \\
& \quad+\int \frac{\partial_{\alpha} \ln z_{\alpha}(\alpha, t)-\partial_{\beta} \ln z_{\beta}(\beta, t)}{z_{\alpha}(\alpha, t)(z(\alpha, t)-z(\beta, t))^{2}}\left(\left(\frac{z(\alpha, t)-z(\beta, t)}{\alpha-\beta}\right)^{2}-z_{\alpha}(\alpha, t) z_{\beta}(\beta, t)\right) d \beta .
\end{aligned}
$$

Therefore for fixed $t \in[0, T]$,

$$
\begin{aligned}
& \int\left|\partial_{\alpha} \mathcal{F}(\alpha, t)\right|^{2} d \alpha \\
& \leq c_{1} \int\left|\partial_{\alpha} V(\alpha, t)\right|^{4} d \alpha \\
& \quad+c_{1} \int\left(\int\left|\frac{V(\alpha, t)-V(\beta, t)}{\alpha-\beta}\right|^{2} d \beta\right)\left(\int\left|\frac{V_{\alpha}(\alpha, t)-V_{\beta}(\beta, t)}{\alpha-\beta}\right|^{2} d \beta\right) d \beta \\
& \leq c_{1} \int\left|\partial_{\alpha} V(\alpha, t)\right|^{4} d \alpha+(2 \pi)^{2} c_{1} \int\left|[V, H] V_{\alpha}\right|\left|\left[V_{\alpha}, H\right] V_{\alpha \alpha}\right| d \alpha \\
& \leq c_{2}\left\|\partial_{\alpha} V(\cdot, t)\right\|_{H^{1 / 2}(\mathbb{R})}^{4} \\
& \quad+c_{2}\left\||D|^{1 / 2} V(\cdot, t)\right\|_{L^{2}(\mathbb{R})}\left\|V_{\alpha}(\cdot, t)\right\|_{L^{2}(\mathbb{R})} \\
& \quad \cdot\left\||D|^{1 / 2} V_{\alpha}(\cdot, t)\right\|_{L^{2}(\mathbb{R})}\left\|V_{\alpha \alpha}(\cdot, t)\right\|_{L^{2}(\mathbb{R})} \\
& \leq c\left(1+\frac{1}{\varepsilon}\left\||D|^{1 / 2} V(\cdot, t)\right\|_{L^{2}(\mathbb{R})}^{2}\right)\|V(\cdot, t)\|_{H^{3 / 2}(\mathbb{R})}^{4}+\varepsilon\left\|V_{\alpha \alpha}(\cdot, t)\right\|_{L^{2}(\mathbb{R})}^{2}
\end{aligned}
$$

for any $\varepsilon>0$. Here $c_{1}, c_{2}$, and $c$ are constants independent of $t \in[0, T]$ but depending on $m$ and $M$. This proves (7.12).

LEMMA 7.4 Let $z^{k}=z^{k}(\alpha, t), k=1,2$, be given, $V_{k}=(1+i) \ln z_{\alpha}^{k}$, and $\ln z_{\alpha}(\alpha, t ; \theta)=\ln z_{\alpha}{ }^{1}(\alpha, t)+\theta\left(\ln z_{\alpha}{ }^{2}(\alpha, t)-\ln z_{\alpha}{ }^{1}(\alpha, t)\right)$ for $0 \leq \theta \leq 1$. Also, let $\mathfrak{a}_{k}=\mathfrak{a}\left(V_{k}\right)$ and $\mathcal{F}_{k}=\mathcal{F}\left(V_{k}\right)$ be defined as in (7.3). Assume that $V_{k} \in$ $L^{\infty}\left([0, T], H^{3 / 2}(\mathbb{R})\right)$ for $k=1,2$, and that there exist constants $m, M>0$ such 
that

$$
\begin{aligned}
& m|\alpha-\beta| \leq|z(\alpha, t ; \theta)-z(\beta, t ; \theta)| \leq M|\alpha-\beta| \\
& \qquad \text { for } \alpha, \beta \in \mathbb{R}, t \in[0, T], 0 \leq \theta \leq 1 .
\end{aligned}
$$

Then there exists a constant $c$, depending on $m$ and $M$ only, such that for a.e. $t \in[0, T]$ and any $\varepsilon>0$,

$$
\begin{aligned}
& \left\|\mathfrak{a}_{1}(\cdot, t)-\mathfrak{a}_{2}(\cdot, t)\right\|_{H^{1 / 2}(\mathbb{R})} \\
& \quad \leq c\left(1+\left\|V_{1}(\cdot, t)\right\|_{H^{1}(\mathbb{R})}\right)\left\|V_{1}(\cdot, t)-V_{2}(\cdot, t)\right\|_{H^{1 / 2}(\mathbb{R})} \\
& \quad \|\left(\mathcal{F}_{1}(\cdot, t)-\mathcal{F}_{2}(\cdot, t) \|_{L^{2}(\mathbb{R})}^{2}\right. \\
& \quad \leq \varepsilon\left\|\partial_{\alpha}\left(V_{1}-V_{2}\right)(\cdot, t)\right\|_{L^{2}(\mathbb{R})}^{2} \\
& \quad+c\left(1+\frac{1}{\varepsilon}\left\||D|^{1 / 2} V_{1}(\cdot, t)\right\|_{L^{2}(\mathbb{R})}^{2}\right)\left\|V_{1}(\cdot, t)\right\|_{H^{3 / 2}(\mathbb{R})}^{2}\left\|\left(V_{1}-V_{2}\right)(\cdot, t)\right\|_{H^{1 / 2}(\mathbb{R})}^{2} \\
& \quad+c\left(1+\frac{1}{\varepsilon}\left\||D|^{1 / 2} V_{2}(\cdot, t)\right\|_{L^{2}(\mathbb{R})}^{2}\right)\left\|V_{2}(\cdot, t)\right\|_{H^{3 / 2}(\mathbb{R})}^{2}\left\|\left(V_{1}-V_{2}\right)(\cdot, t)\right\|_{H^{1 / 2}(\mathbb{R})}^{2}
\end{aligned}
$$

PROOF: The proof of (7.16) is similar to that for Lemma 7.1(iii) and straightforward; we omit the details. We have

$$
\begin{aligned}
& \frac{2 \pi i}{1-i}\left(\mathcal{F}_{1}-\mathcal{F}_{2}\right) \\
& =\left(\frac{\pi}{\left|z_{\alpha}^{1}\right|^{2}}-\frac{\pi}{\left|z_{\alpha}^{2}\right|^{2}}\right)|D| \ln z_{\alpha}{ }^{1}+\left(\frac{1}{\bar{z}_{\alpha}^{1}}-\frac{1}{\bar{z}_{\alpha}^{2}}\right) \int \frac{-z_{\alpha}^{1}+z_{\beta}^{1}}{\left(z^{1}(\alpha, t)-z^{1}(\beta, t)\right)^{2}} d \beta \\
& \quad+\frac{\pi}{\left|z_{\alpha}^{2}\right|^{2}}|D|\left(\ln z_{\alpha}{ }^{1}-\ln z_{\alpha}{ }^{2}\right)+\frac{1}{\bar{z}_{\alpha}^{2}} \int \frac{\left(-z_{\alpha}^{1} / z_{\beta}^{1}+z_{\alpha}^{2} / z_{\beta}^{2}\right) z_{\beta}^{2}}{\left(z^{2}(\alpha, t)-z^{2}(\beta, t)\right)^{2}} d \beta \\
& \quad+\frac{1}{\bar{z}_{\alpha}^{2}}\left(\int \frac{\left(-z_{\alpha}^{1} / z_{\beta}^{1}+1\right) z_{\beta}^{1}}{\left(z^{1}(\alpha, t)-z^{1}(\beta, t)\right)^{2}} d \beta-\int \frac{\left(-z_{\alpha}^{1} / z_{\beta}^{1}+1\right) z_{\beta}^{2}}{\left(z^{2}(\alpha, t)-z^{2}(\beta, t)\right)^{2}} d \beta\right) .
\end{aligned}
$$

Now

$$
\begin{aligned}
& \int \frac{\left(-z_{\alpha}^{1}(\alpha, t) / z_{\beta}^{1}(\beta, t)+1\right) z_{\beta}^{1}(\beta, t)}{\left(z^{1}(\alpha, t)-z^{1}(\beta, t)\right)^{2}} d \beta \\
& -\int \frac{\left(-z_{\alpha}^{1}(\alpha, t) / z_{\beta}^{1}(\beta, t)+1\right) z_{\beta}^{2}(\beta, t)}{\left(z^{2}(\alpha, t)-z^{2}(\beta, t)\right)^{2}} d \beta \\
& \quad=\int \frac{z_{\alpha}^{1}(\alpha, t)}{z^{1}(\alpha, t)-z^{1}(\beta, t)} \partial_{\beta} \frac{1}{z_{\beta}^{1}(\beta, t)} d \beta-\int \frac{z_{\alpha}^{1}(\alpha, t)}{z^{2}(\alpha, t)-z^{2}(\beta, t)} \partial_{\beta} \frac{1}{z_{\beta}^{1}(\beta, t)} d \beta \\
& \quad=\left(C_{z^{1}}-C_{z^{2}}\left(\partial_{\alpha} \frac{1}{z_{\alpha}^{1}(\alpha, t)}\right)-\frac{z_{\alpha}^{1}(\alpha, t)-z_{\alpha}^{2}(\alpha, t)}{z_{\alpha}^{2}(\alpha, t)} C_{z^{2}}\left(\partial_{\alpha} \frac{1}{z_{\alpha}^{1}(\alpha, t)}\right),\right.
\end{aligned}
$$


and

$$
\begin{aligned}
& \frac{\pi}{z_{\alpha}^{2}}|D|\left(\ln z_{\alpha}^{1}-\ln z_{\alpha}^{2}\right)+\int \frac{\left(-z_{\alpha}^{1} / z_{\beta}^{1}+z_{\alpha}^{2} / z_{\beta}^{2}\right) z_{\beta}^{2}}{\left(z^{2}(\alpha, t)-z^{2}(\beta, t)\right)^{2}} d \beta \\
& =\int \frac{\left(-z_{\alpha}^{1} / z_{\beta}^{1}+z_{\alpha}^{2} / z_{\beta}^{2}+\ln \left(z_{\alpha}^{1} / z_{\beta}^{1}\right)-\ln \left(z_{\alpha}^{2} / z_{\beta}^{2}\right)\right) z_{\beta}^{2}}{\left(z^{2}(\alpha, t)-z^{2}(\beta, t)\right)^{2}} d \beta \\
& \quad+\int \frac{\ln z_{\alpha}^{1}-\ln z_{\alpha}^{2}-\ln z_{\beta}^{1}+\ln z_{\beta}^{2}}{\left(z^{2}(\alpha, t)-z^{2}(\beta, t)\right)^{2} z_{\alpha}^{2}(\alpha, t)}\left(-z_{\beta}^{2}(\beta, t) z_{\alpha}^{2}(\alpha, t)+\left(\frac{z^{2}(\alpha, t)-z^{2}(\beta, t)}{\alpha-\beta}\right)^{2}\right) d \beta
\end{aligned}
$$

Therefore for fixed $t \in[0, T]$,

$$
\begin{aligned}
& \int\left|\left(\mathcal{F}_{1}-\mathcal{F}_{2}\right)(\alpha, t)\right|^{2} d \alpha \\
& \leq c\left(\int\left|\left(V_{1}-V_{2}\right)(\alpha, t)\right|^{4} d \alpha\right)^{1 / 2}\left(\int\left|\partial_{\alpha} V_{1}(\alpha, t)\right|^{4} d \alpha\right)^{1 / 2} \\
& \quad+c \int\left(\int\left|\frac{\left(V_{2}-V_{1}\right)(\alpha, t)-\left(V_{2}-V_{1}\right)(\beta, t)}{\alpha-\beta}\right|^{2} d \beta\right)\left(\int\left|\frac{V_{2}(\alpha, t)-V_{2}(\beta, t)}{\alpha-\beta}\right|^{2} d \beta\right) d \alpha \\
& \quad+c \int\left(\int\left|\frac{\left(V_{2}-V_{1}\right)(\alpha, t)-\left(V_{2}-V_{1}\right)(\beta, t)}{\alpha-\beta}\right|^{2} d \beta\right)\left(\int\left|\frac{V_{1}(\alpha, t)-V_{1}(\beta, t)}{\alpha-\beta}\right|^{2} d \beta\right) d \alpha \\
& \quad+c\left\||D|^{1 / 2}\left(V_{2}-V_{1}\right)(\cdot, t)\right\|_{L^{2}(\mathbb{R})}^{2}\left\|\partial_{\alpha} V_{1}(\cdot, t)\right\|_{L^{2}(\mathbb{R})}^{2} .
\end{aligned}
$$

From here (7.17) follows similarly as in (7.14).

\subsection{Linear Equation}

Some versions of Lemmas 7.5 through 7.7 are classical results in linear PDEs and may be found in [6].

Consider first the linear equation

$$
\left\{\begin{array}{l}
u_{t}+a|D| u-\epsilon \partial_{\alpha}^{2} u=f \\
u(\cdot, 0)=u_{0}
\end{array}\right.
$$

in $\mathbb{R} \times[0, T]$, where $|D|=H \partial_{\alpha}=\left|\partial_{\alpha}\right|, \epsilon>0$.

Let $s>0$. We use the notation that $|u|_{H^{s}(\mathbb{R})}=\left(\|u\|_{L^{2}(\mathbb{R})}^{2}+\left\||D|^{s} u\right\|_{L^{2}(\mathbb{R})}^{2}\right)^{1 / 2}$ for $u \in H^{s}(\mathbb{R})$ and $|u|_{H^{0}(\mathbb{R})}=\|u\|_{L^{2}(\mathbb{R})}$. We have the following:

LEMMA 7.5 Let $\epsilon>0, T>0$, and $u_{0} \in H^{2}(\mathbb{R})$. Assume that $a-1 \in$ $L^{\infty}\left([0, T], H^{3 / 2}(\mathbb{R})\right)$ and $f \in L^{2}\left([0, T], H^{1}(\mathbb{R})\right)$. Then the initial value problem (7.18) has a unique solution $u \in C\left([0, T], H^{2}(\mathbb{R})\right) \cap L^{2}\left([0, T], H^{3}(\mathbb{R})\right)$.

Proof: Let $\epsilon>0$. We know that for any given $f \in L^{2}\left([0, T], H^{1}(\mathbb{R})\right)$ and $u_{0} \in H^{2}(\mathbb{R})$, the initial value problem

$$
\left\{\begin{array}{l}
u_{t}-\epsilon \partial_{\alpha}^{2} u=f \\
u(\alpha, 0)=u_{0}(\alpha)
\end{array}\right.
$$

has a unique solution $u \in C\left([0, T], H^{2}(\mathbb{R})\right) \cap L^{2}\left([0, T], H^{3}(\mathbb{R})\right)$ such that for each $t \in[0, T]$, 


$$
\begin{aligned}
|u(\cdot, t)|_{H^{2}(\mathbb{R})}^{2}+\epsilon \int_{0}^{t} e^{t-\tau}\left|\partial_{\alpha} u(\cdot, \tau)\right|_{H^{2}(\mathbb{R})}^{2} d \tau & \\
& \leq e^{t}\left|u_{0}\right|_{H^{2}(\mathbb{R})}^{2}+c \int_{0}^{t} e^{t-\tau}|f(\cdot, \tau)|_{H^{1}(\mathbb{R})}^{2} d \tau
\end{aligned}
$$

where $c=\max (1,1 / \epsilon)$. We solve the initial value problem (7.18) using the fixed point theorem. Let $u^{0}(\alpha, t)=u_{0}(\alpha)$ for $t \in[0, T]$; we construct a sequence $u^{j}$ through

$$
\left\{\begin{array}{l}
u_{t}^{j+1}-\epsilon \partial_{\alpha}^{2} u^{j+1}=f-a|D| u^{j} \\
u(\alpha, 0)=u_{0}(\alpha)
\end{array}\right.
$$

Therefore $u^{j} \in C\left([0, T], H^{2}(\mathbb{R})\right) \cap L^{2}\left([0, T], H^{3}(\mathbb{R})\right)$ and

$$
\left\{\begin{array}{l}
\left(u^{j+1}-u^{j}\right)_{t}-\epsilon \partial_{\alpha}^{2}\left(u^{j+1}-u^{j}\right)=-a|D|\left(u^{j}-u^{j-1}\right) \\
\left(u^{j+1}-u^{j}\right)(\cdot, 0)=0
\end{array}\right.
$$

Applying (7.19), we get

$$
\begin{aligned}
& \left|\left(u^{j+1}-u^{j}\right)(\cdot, t)\right|_{H^{2}(\mathbb{R})}^{2}+\epsilon \int_{0}^{t} e^{t-\tau}\left|\partial_{\alpha}\left(u^{j+1}-u^{j}\right)(\cdot, \tau)\right|_{H^{2}(\mathbb{R})}^{2} d \tau \\
& \quad \leq c \int_{0}^{t} e^{t-\tau}|a| D\left|\left(u^{j}-u^{j-1}\right)(\cdot, \tau)\right|_{H^{1}(\mathbb{R})}^{2} d \tau \\
& \quad \leq c_{1} \int_{0}^{t}\left|\left(u^{j}-u^{j-1}\right)(\cdot, \tau)\right|_{H^{2}(\mathbb{R})}^{2} d \tau
\end{aligned}
$$

Here in the last step we applied Lemma 7.1(i). The constant $c_{1}$ depends on $\|a-1\|_{L^{\infty}\left([0, T], H^{3 / 2}(\mathbb{R})\right)}$ and $c$. This shows that $u^{j}$ is a Cauchy sequence in

$$
C\left([0, T], H^{2}(\mathbb{R})\right) \cap L^{2}\left([0, T], H^{3}(\mathbb{R})\right) .
$$

Therefore, there is a function

$$
u \in C\left([0, T], H^{2}(\mathbb{R})\right) \cap L^{2}\left([0, T], H^{3}(\mathbb{R})\right)
$$

such that $u^{j} \rightarrow u$ in $C\left([0, T], H^{2}(\mathbb{R})\right) \cap L^{2}\left([0, T], H^{3}(\mathbb{R})\right)$. The uniqueness can be obtained similarly; we omit it. This proves Lemma 7.5.

LemMA 7.6 Let $\epsilon \geq 0, T>0$, and $u_{0} \in H^{3 / 2}(\mathbb{R})$. Assume that $a-1 \in$ $L^{\infty}\left([0, T], H^{3 / 2}(\mathbb{R})\right), f \in L^{2}\left([0, T], H^{1}(\mathbb{R})\right)$, and $a \geq c_{0}$ for some constant $c_{0}>0$. If for some real number $s$,

$$
u \in L^{\infty}\left([0, T], H^{3 / 2}(\mathbb{R})\right) \cap C^{1}\left([0, T], H^{s}(\mathbb{R})\right) \cap L^{2}\left([0, T], H^{2}(\mathbb{R})\right)
$$


and $\epsilon|D|^{5 / 2} u \in L^{2}(\mathbb{R} \times[0, T])$ is a solution for (7.18), then $u \in C([0, T]$, $\left.H^{3 / 2}(\mathbb{R})\right)$, and for every $t \in[0, T], j=0,1$,

$$
\begin{aligned}
& |u(\cdot, t)|_{H^{j+1 / 2}(\mathbb{R})}^{2}+c_{0} \int_{0}^{t} e^{\kappa(t-\tau)}\left\|\partial_{\alpha}^{j+1} u(\cdot, \tau)\right\|_{L^{2}(\mathbb{R})}^{2} d \tau \\
& +2 \epsilon \int_{0}^{t} e^{\kappa(t-\tau)}\left|\partial_{\alpha} u(\cdot, \tau)\right|_{H^{j+1 / 2}(\mathbb{R})}^{2} d \tau \\
& \quad \leq e^{\kappa t}\left|u_{0}\right|_{H^{j+1 / 2}(\mathbb{R})}^{2}+\left(1+\frac{2}{c_{0}}\right) \int_{0}^{t} e^{\kappa(t-\tau)}|f(\cdot, \tau)|_{H^{j}(\mathbb{R})}^{2} d \tau
\end{aligned}
$$

where $\kappa=c\left(\|a-1\|_{L^{\infty}\left([0, T], H^{3 / 2}(\mathbb{R})\right)}^{2}+1\right)$ and $c$ is a constant depending on $c_{0}$.

PROOF:

Step 1. Assume that the solution $u \in C^{1}\left([0, T], H^{10}(\mathbb{R})\right)$. From equation (7.18), we have

$$
\begin{aligned}
& \left.\left.\frac{d}{d t} \int|| D\right|^{1 / 2}\left(\partial_{\alpha} u(\alpha, t)\right)\right|^{2} d \alpha \\
& \quad=2 \int|D| \partial_{\alpha} u(\alpha, t)\left(\partial_{\alpha} u(\alpha, t)\right)_{t} d \alpha \\
& \quad=2 \int\left(\epsilon \partial_{\alpha}^{3} u-a|D| \partial_{\alpha} u\right)|D| \partial_{\alpha} u d \alpha+2 \int\left(\partial_{\alpha} f-\partial_{\alpha} a|D| u\right)|D| \partial_{\alpha} u d \alpha .
\end{aligned}
$$

Therefore

$$
\begin{aligned}
\left.\left.\frac{d}{d t} \int|| D\right|^{1 / 2}\left(\partial_{\alpha} u(\alpha, t)\right)\right|^{2} d \alpha \\
+\left.\left.2 \epsilon \int|| D\right|^{5 / 2} u(\alpha, t)\right|^{2} d \alpha+2 \int a\left|\partial_{\alpha}^{2} u(\alpha, t)\right|^{2} d \alpha \\
\leq c_{0} \int\left|\partial_{\alpha}^{2} u(\alpha, t)\right|^{2} d \alpha \\
\quad+\frac{2}{c_{0}} \int\left|\partial_{\alpha} f\right|^{2} d \alpha+\frac{2}{c_{0}}\left(\int\left|\partial_{\alpha} a\right|^{4} d \alpha\right)^{1 / 2}\left(\int\left|\partial_{\alpha} u\right|^{4} d \alpha\right)^{1 / 2} \\
\leq c_{0} \int\left|\partial_{\alpha}^{2} u(\alpha, t)\right|^{2} d \alpha \\
\quad+\frac{2}{c_{0}} \int\left|\partial_{\alpha} f\right|^{2} d \alpha+c_{1}\|a\|_{L^{\infty}\left([0, T], H^{3 / 2}(\mathbb{R})\right)}^{2}|u(\cdot, t)|_{H^{3 / 2}(\mathbb{R})}^{2} .
\end{aligned}
$$

Here in the last step, we applied Lemma 7.1(i). The constant $c_{1}$ is universal. We also have from (7.18) that

$$
\begin{aligned}
\left.\left.\frac{d}{d t} \int|| D\right|^{1 / 2} u(\alpha, t)\right|^{2} d \alpha & =2 \int|D| u(\alpha, t) u_{t}(\alpha, t) d \alpha \\
& =2 \int\left(\epsilon \partial_{\alpha}^{2} u-a|D| u\right)|D| u d \alpha+2 \int f|D| u d \alpha
\end{aligned}
$$


therefore

$$
\begin{aligned}
& \left.\left.\frac{d}{d t} \int|| D\right|^{1 / 2} u(\alpha, t)\right|^{2} d \alpha+\left.\left.2 \epsilon \int|| D\right|^{3 / 2} u(\alpha, t)\right|^{2} d \alpha \\
& \quad+2 \int a\left|\partial_{\alpha} u(\alpha, t)\right|^{2} d \alpha \\
& \quad \leq \frac{c_{0}}{2} \int\left|\partial_{\alpha} u(\alpha, t)\right|^{2} d \alpha+\frac{2}{c_{0}} \int|f(\alpha, t)|^{2} d \alpha
\end{aligned}
$$

and

$$
\begin{aligned}
& \frac{d}{d t} \int|u(\alpha, t)|^{2} d \alpha+2 \epsilon \int\left|\partial_{\alpha} u(\alpha, t)\right|^{2} d \alpha \\
& \quad=2 \int u(\alpha, t)\left(u_{t}(\alpha, t)-\epsilon \partial_{\alpha}^{2} u(\alpha, t)\right) d \alpha=2 \int u(-a|D| u+f) d \alpha \\
& \quad \leq\left(1+\frac{2\|a\|_{L^{\infty}(R \times[0, T])}^{2}}{c_{0}}\right) \int|u|^{2} d \alpha+\int|f|^{2} d \alpha+\frac{c_{0}}{2} \int\left|\partial_{\alpha} u\right|^{2} d \alpha .
\end{aligned}
$$

Summing up (7.22) and (7.24) and then (7.23) and (7.24), we get, for $j=0,1$,

$$
\begin{aligned}
& \frac{d}{d t}|u(\cdot, t)|_{H^{j+1 / 2}(\mathbb{R})}^{2}+2 \epsilon\left|\partial_{\alpha} u(\cdot, t)\right|_{H^{j+1 / 2}(\mathbb{R})}^{2}+c_{0}\left\|\partial_{\alpha}^{j+1} u(\cdot, t)\right\|_{L^{2}(\mathbb{R})}^{2} \\
& \quad \leq \kappa|u(\cdot, t)|_{H^{j+1 / 2}(\mathbb{R})}^{2}+\left(1+\frac{2}{c_{0}}\right)|f(\cdot, t)|_{H^{j}(\mathbb{R})}^{2}
\end{aligned}
$$

where $\kappa=c_{2}\left(\|a\|_{L^{\infty}\left([0, T], H^{3 / 2}(\mathbb{R})\right)}^{2}+1\right)$ and $c_{2}$ is a constant depending on $c_{0}$. Inequality (7.20) follows from (7.25) through a standard ODE procedure.

Step 2. Assume that $u$ satisfies the assumption of Lemma 7.6. We claim that $u(\cdot, t) \in H^{3 / 2}(\mathbb{R})$ for each $t \in[0, T]$. From $u \in L^{\infty}\left([0, T], H^{3 / 2}(\mathbb{R})\right)$, we know that $u(\cdot, t) \in H^{3 / 2}(\mathbb{R})$ for a.e. $t \in[0, T]$. On the other hand, from $u \in$ $C^{1}\left([0, T], H^{s}(\mathbb{R})\right)$ for some $s \in \mathbb{R}$, we have $u(\cdot, t) \rightarrow u\left(\cdot, t_{0}\right)$ weakly in $H^{s}(\mathbb{R})$ as $t \rightarrow t_{0}$ in $[0, T]$. For any given $t_{0} \in[0, T]$, take a sequence $t_{j} \in[0, T]$ such that $u\left(\cdot, t_{j}\right)$ forms a bounded sequence in $H^{3 / 2}(\mathbb{R})$ and $t_{j} \rightarrow t_{0}$ as $j \rightarrow \infty$. The weak limit $u\left(\cdot, t_{0}\right)$ of the sequence $u\left(\cdot, t_{j}\right)$ is also in $H^{3 / 2}(\mathbb{R})$. This proves our claim.

Let $\phi \in C_{0}^{\infty}(\mathbb{R})$ and $\phi^{\delta}(\alpha)=(1 / \delta) \phi(\alpha / \delta)$. We have $u * \phi^{\delta} \in C^{1}([0, T]$, $\left.H^{10}(\mathbb{R})\right)$ and $u * \phi^{\delta}$ satisfies

(7.26) $\left(u * \phi^{\delta}\right)_{t}+a|D|\left(u * \phi^{\delta}\right)-\epsilon \partial_{\alpha}^{2}\left(u * \phi^{\delta}\right)=f * \phi^{\delta}+a|D|\left(u * \phi^{\delta}\right)-(a|D| u) * \phi^{\delta}$.

Notice that $a|D| u, a|D| \partial_{\alpha} u, \partial_{\alpha} a|D| u \in L^{2}(\mathbb{R} \times[0, T])$, so

$$
\int_{0}^{T}\left|(a|D| u) * \phi^{\delta}(\cdot, t)-a\right| D|u(\cdot, t)|_{H^{1}(\mathbb{R})}^{2} d t \rightarrow 0
$$


as $\delta \rightarrow 0$. On the other hand,

$$
\begin{aligned}
& \int_{0}^{T}|a| D\left|\left(u * \phi^{\delta}(\cdot, t)\right)-a\right| D|u(\cdot, t)|_{H^{1}(\mathbb{R})}^{2} d t \\
& \quad \leq 2\|a\|_{L^{\infty}(R \times[0, T])}^{2} \int_{0}^{T}\left|\left(u * \phi^{\delta}(\cdot, t)-u(\cdot, t)\right)\right|_{H^{2}(\mathbb{R})}^{2} d t \\
& \quad+2 \iint\left|\partial_{\alpha} a\right| D\left|\left(u * \phi^{\delta}(\cdot, t)-u(\cdot, t)\right)\right|^{2} d \alpha d t .
\end{aligned}
$$

Apply Holder's inequality and Lemma 7.1(i) to the last term in inequality (7.27), we get

$$
\int_{0}^{T}|a| D\left|\left(u * \phi^{\delta}(\cdot, t)\right)-a\right| D|u(\cdot, t)|_{H^{1}(\mathbb{R})}^{2} d t \rightarrow 0
$$

as $\delta \rightarrow 0$; therefore

$$
\int_{0}^{T}\left|(a|D| u) * \phi^{\delta}(\cdot, t)-a\right| D\left|\left(u * \phi^{\delta}(\cdot, t)\right)\right|_{H^{1}(\mathbb{R})}^{2} d t \rightarrow 0
$$

as $\delta \rightarrow 0$.

Let $\tilde{f}=f * \phi^{\delta}+a|D|\left(u * \phi^{\delta}\right)-(a|D| u) * \phi^{\delta}$. Applying the result in Step 1 to $u * \phi^{\delta}$, we get

$$
\begin{aligned}
& \left|u * \phi^{\delta}(\cdot, t)\right|_{H^{j+1 / 2}(\mathbb{R})}^{2}+c_{0} \int_{0}^{t} e^{\kappa(t-\tau)}\left\|\partial_{\alpha}^{j+1} u * \phi^{\delta}(\cdot, \tau)\right\|_{L^{2}(\mathbb{R})}^{2} d \tau \\
& +2 \epsilon \int_{0}^{t} e^{\kappa(t-\tau)}\left|\partial_{\alpha} u * \phi^{\delta}(\cdot, \tau)\right|_{H^{j+1 / 2}(\mathbb{R})}^{2} d \tau \\
& \quad \leq e^{\kappa t}\left|u_{0} * \phi^{\delta}\right|_{H^{j+1 / 2}(\mathbb{R})}^{2}+\left(1+\frac{2}{c_{0}}\right) \int_{0}^{t} e^{\kappa(t-\tau)}|\tilde{f}(\cdot, \tau)|_{H^{j}(\mathbb{R})}^{2} d \tau .
\end{aligned}
$$

Let $\delta \rightarrow 0$ on both sides of (7.29); we get (7.20) for every $t \in[0, T]$.

We now prove that $u \in C\left([0, T], H^{3 / 2}(\mathbb{R})\right)$. Applying (7.21) and (7.24) to $u * \phi^{\delta}$ and (7.26), we get

$$
\begin{array}{rl}
\left|\frac{d}{d t}\right| u & \left.* \phi^{\delta}(\cdot, t)\right|_{H^{3 / 2}(\mathbb{R})} ^{2} \mid \\
\leq & 2 \epsilon\left|\partial_{\alpha} u * \phi^{\delta}(\cdot, t)\right|_{H^{3 / 2}(\mathbb{R})}^{2} \\
& +\left(2\|a\|_{L^{\infty}(R \times[0, T])}+2\right)\left\|\partial_{\alpha}^{2} u * \phi^{\delta}(\cdot, t)\right\|_{L^{2}(\mathbb{R})}+c\left|u * \phi^{\delta}(\cdot, t)\right|_{H^{3 / 2}(\mathbb{R})}^{2} \\
& +|\tilde{f}|_{H^{1}(\mathbb{R})}^{2},
\end{array}
$$

where $c$ is a constant depending on $\|a-1\|_{L^{\infty}\left([0, T], H^{3 / 2}(\mathbb{R})\right)}$. Integrate both sides of (7.30) with respect to $t$ from $t_{0}$ to $t$, and then let $\delta \rightarrow 0$. We obtain

$$
\left.|| u(\cdot, t)\right|_{H^{3 / 2}(\mathbb{R})} ^{2}-\left|u\left(\cdot, t_{0}\right)\right|_{H^{3 / 2}(\mathbb{R})}^{2}|\leq| \int_{t_{0}}^{t} g(t) d t \mid
$$


for some $g \in L^{1}[0, T]$. This proves that $|u(\cdot, t)|_{H^{3 / 2}(\mathbb{R})}^{2} \rightarrow\left|u\left(\cdot, t_{0}\right)\right|_{H^{3 / 2}(\mathbb{R})}^{2}$ as $t \rightarrow t_{0}$ in $[0, T]$. Since $u(\cdot, t) \rightarrow u\left(\cdot, t_{0}\right)$ weakly, therefore

$$
\left|u(\cdot, t)-u\left(\cdot, t_{0}\right)\right|_{H^{3 / 2}(\mathbb{R})}^{2} \rightarrow 0
$$

as $t \rightarrow t_{0}$ in $[0, T]$.

LEMMA 7.7 Assume that $a \geq c_{0}$ for some constant $c_{0}>0, a-1 \in C([0, T]$, $\left.H^{3 / 2}(\mathbb{R})\right)$, and $f \in L^{2}\left([0, T], H^{1}(\mathbb{R})\right) \cap C\left([0, T], H^{1 / 2}(\mathbb{R})\right)$. Let $u_{0} \in H^{3 / 2}(\mathbb{R})$.

Then the initial value problem

$$
\left\{\begin{array}{l}
u_{t}+a|D| u=f \\
u(\cdot, 0)=u_{0}
\end{array}\right.
$$

has a unique solution $u \in C\left([0, T], H^{3 / 2}(\mathbb{R})\right) \cap C^{1}\left([0, T], H^{1 / 2}(\mathbb{R})\right) \cap L^{2}([0, T]$, $H^{2}(\mathbb{R})$ ).

Proof: Notice that (7.31) is the same as (7.18) with $\epsilon=0$. Let $\epsilon>0$, $u^{\epsilon} \in C\left([0, T], H^{2}(\mathbb{R})\right) \cap L^{2}\left([0, T], H^{3}(\mathbb{R})\right)$ be the unique solution of

$$
\left\{\begin{array}{l}
u_{t}^{\epsilon}+a|D| u^{\epsilon}-\epsilon \partial_{\alpha}^{2} u^{\epsilon}=f \\
u^{\epsilon}(\cdot, 0)=u_{0} * \phi^{\epsilon}
\end{array}\right.
$$

From equation (7.32), we know $u^{\epsilon} \in C^{1}\left([0, T], L^{2}(\mathbb{R})\right)$. From (7.20), $j=1$, we have that $\left\{u^{\epsilon}: \epsilon>0\right\}$ is a bounded set in $C\left([0, T], H^{3 / 2}(\mathbb{R})\right) \cap L^{2}\left([0, T], H^{2}(\mathbb{R})\right)$. Now

$$
\left\{\begin{array}{l}
\left(u^{\epsilon}-u^{\delta}\right)_{t}+a|D|\left(u^{\epsilon}-u^{\delta}\right)-\epsilon \partial_{\alpha}^{2}\left(u^{\epsilon}-u^{\delta}\right)=(\epsilon-\delta) \partial_{\alpha}^{2} u^{\delta} \\
\left(u^{\epsilon}-u^{\delta}\right)(\cdot, 0)=u_{0} * \phi^{\epsilon}-u_{0} * \phi^{\delta}
\end{array}\right.
$$

Applying (7.20), $j=0$, to $u^{\epsilon}-u^{\delta}$, we have

$$
\begin{aligned}
& \left|\left(u^{\epsilon}-u^{\delta}\right)(\cdot, t)\right|_{H^{1 / 2}(\mathbb{R})}^{2}+c_{0} \int_{0}^{t} e^{\kappa(t-\tau)}\left\|\partial_{\alpha}\left(u^{\epsilon}-u^{\delta}\right)(\cdot, \tau)\right\|_{L^{2}(\mathbb{R})}^{2} d \tau \\
& \leq e^{\kappa t}\left|u_{0} *\left(\phi^{\epsilon}-\phi^{\delta}\right)\right|_{H^{1 / 2}(\mathbb{R})}^{2}+c(\epsilon-\delta)^{2} \int_{0}^{t} \int\left|\partial_{\alpha}^{2} u^{\delta}(\alpha, \tau)\right|^{2} d \alpha d \tau .
\end{aligned}
$$

Therefore $u^{\epsilon}$ is a Cauchy sequence in $C\left([0, T], H^{1 / 2}(\mathbb{R})\right) \cap L^{2}\left([0, T], H^{1}(\mathbb{R})\right)$, and there is a $u \in C\left([0, T], H^{1 / 2}(\mathbb{R})\right) \cap L^{2}\left([0, T], H^{1}(\mathbb{R})\right)$ such that $u^{\epsilon} \rightarrow u$ in $C\left([0, T], H^{1 / 2}(\mathbb{R})\right) \cap L^{2}\left([0, T], H^{1}(\mathbb{R})\right)$ as $\epsilon \rightarrow 0$.

Letting $\epsilon \rightarrow 0$ in (7.32), we get $u$ is a solution of (7.31). Moreover, from (7.31), we get $u \in C^{1}\left([0, T], H^{-1}(\mathbb{R})\right)$ (because $\left.a|D| u=\partial_{\alpha}(a H u)-\partial_{\alpha} a H u\right)$. On the other hand, since $\left\{u^{\epsilon}: \epsilon>0\right\}$ is a bounded set in $C\left([0, T], H^{3 / 2}(\mathbb{R})\right) \cap$ $L^{2}\left([0, T], H^{2}(\mathbb{R})\right)$ and $u$ is the weak limit of $u^{\epsilon}$, we have

$$
u \in L^{\infty}\left([0, T], H^{3 / 2}(\mathbb{R})\right) \cap L^{2}\left([0, T], H^{2}(\mathbb{R})\right) .
$$

From Lemma 7.6 and equation (7.31), we obtain

$$
u \in C\left([0, T], H^{3 / 2}(\mathbb{R})\right) \cap C^{1}\left([0, T], H^{1 / 2}(\mathbb{R})\right) .
$$


This proves the existence of solutions of (7.31). The uniqueness follows from (7.20).

\subsection{The Quasi-Linear System (7.5)-(7.3)}

We now prove that the quasi-linear system is solvable for a finite time period $[0, T]$ for any given $w(\alpha, 0)=w_{0}(\alpha) \in H^{3 / 2}(\mathbb{R})$. We will use the iteration method and the fixed point theorm.

THEOREM 7.8 Assume that $w_{0} \in H^{3 / 2}(\mathbb{R})$. Then there is a $T=T\left(\|w\|_{H^{3 / 2}(\mathbb{R})}\right)>$ 0 such that the quasi-linear system (7.5)-(7.3) has a solution

$$
V \in C\left([0, T], H^{3 / 2}(\mathbb{R})\right) \cap C^{1}\left([0, T], H^{1 / 2}(\mathbb{R})\right) \cap L^{2}\left([0, T], H^{2}(\mathbb{R})\right)
$$

satisfying $w(\cdot, 0)=w_{0}(\cdot)$. Moreover, $\ln z_{\alpha}=V /(1+i)$ defines a chord-arc curve $z=z(\alpha, t)$ for each fixed $t \in[0, T]$, and there are constants $m, M>0$ independent of $t$ such that

$$
m|\alpha-\beta| \leq|z(\alpha, t)-z(\beta, t)| \leq M|\alpha-\beta| \quad \text { for } \alpha, \beta \in \mathbb{R}, t \in[0, T] .
$$

We prove by iteration. Notice that system (7.5) consists of two evolutionary equations, a forward equation for $w$ and a backward equation for $v$, while only the data $w(\cdot, 0)=w_{0}$ is given. Therefore we are allowed to prescribe data for $v$ at some later time $t=T$ ( $T$ to be determined) and solve for $v$ backwards.

Let $v_{T} \in H^{3 / 2}(\mathbb{R})$ be such that

$$
\ln \xi_{\alpha}=\frac{v_{T}+i w_{0}}{1+i}
$$

defines a chord-arc curve $\xi=\xi(\alpha)$, with

$$
m_{0}|\alpha-\beta| \leq|\xi(\alpha)-\xi(\beta)| \leq M_{0}|\alpha-\beta|, \quad \alpha, \beta \in \mathbb{R},
$$

for some constants $m_{0}, M_{0}>0$. There are many choices of $v_{T}$, one of which is $v_{T}=w_{0}$. In this case, we have $\ln \xi_{\alpha}=w_{0}$; that is, $\ln \xi_{\alpha}$ is a real-valued function in $H^{3 / 2}(\mathbb{R}) \subset L^{\infty}(\mathbb{R})$. So $\xi=\xi(\alpha)$ is a straight line satisfying (7.33). We want to solve system (7.5)-(7.3) with

$$
w(\alpha, 0)=w_{0}(\alpha), \quad v(\alpha, T)=v_{T}(\alpha), \quad \alpha \in \mathbb{R},
$$

where $T>0$ is to be determined. Assume

$$
\left\|w_{0}\right\|_{H^{3 / 2}(\mathbb{R})} \leq N, \quad\left\|v_{T}\right\|_{H^{3 / 2}(\mathbb{R})} \leq N .
$$

Let

$$
P^{t}(x)=\frac{t}{\pi\left(x^{2}+t^{2}\right)}
$$

be the Poisson kernel. Take

$$
V^{0}=v^{0}+i w^{0}=P^{T-t} * v_{T}+i P^{t} * w_{0} \quad \text { for } t \in[0, T] .
$$


We have $V^{0} \in C\left([0, T], H^{3 / 2}(\mathbb{R})\right) \cap L^{2}\left([0, T], H^{2}(\mathbb{R})\right)$ and

$$
\begin{aligned}
\int_{0}^{t} \int\left|\partial_{\alpha}^{2} V^{0}(\alpha, t)\right|^{2} d \alpha d t & \leq \frac{1}{2}\left(\left\||D|^{3 / 2} v_{T}\right\|_{L^{2}(\mathbb{R})}^{2}+\left\||D|^{3 / 2} w_{0}\right\|_{L^{2}(\mathbb{R})}^{2}\right) \leq N^{2}, \\
\left|V^{0}(\cdot, t)\right|_{H^{3 / 2}(\mathbb{R})} & \leq\left|v_{T}\right|_{H^{3 / 2}(\mathbb{R})}+\left|w_{0}\right|_{H^{3 / 2}(\mathbb{R})} \leq 2 N \quad \text { for } t \in[0, T] .
\end{aligned}
$$

Let $z^{0}=z^{0}(\alpha, t)$ be defined by $(1+i) \ln z_{\alpha}{ }^{0}(\alpha, t)=V^{0}(\alpha, t)$ for $(\alpha, t) \in$ $\mathbb{R} \times[0, T]$. Notice that we need one more bit of information such as $z^{0}(0, t)$ to completely determine $z^{0}(\alpha, t)$. We have

$$
\ln z_{\alpha}^{0}(\alpha, t)=\ln \xi_{\alpha}(\alpha)+b^{0}(\alpha, t)
$$

where $b^{0}(\alpha, t)=[(1-i) / 2]\left(v^{0}(\alpha, t)-v_{T}(\alpha)\right)+i\left(w^{0}(\alpha, t)-w_{0}(\alpha)\right)$. Notice that

$$
\|f\|_{L^{\infty}(\mathbb{R})} \leq 2|f|_{H^{3 / 2}(\mathbb{R})}
$$

for any function $f$ defined on $\mathbb{R}$. Therefore from well-known properties of the Poisson kernel, we have for $t \in[0, T]$,

$$
\begin{aligned}
\left\|\operatorname{Re} b^{0}(\cdot, t)\right\|_{L^{\infty}(\mathbb{R})} & \leq \frac{1}{2}\left(\left\|v^{0}-v_{T}\right\|_{L^{\infty}}+\left\|w^{0}-w_{0}\right\|_{L^{\infty}}\right) \\
& \leq\left\|v_{T}\right\|_{L^{\infty}}+\left\|w_{0}\right\|_{L^{\infty}} \leq 4 N
\end{aligned}
$$

and from Lemma 7.1(ii) and Plancherel's theorem,

$$
\begin{aligned}
& \left\|b^{0}(\cdot, t)\right\|_{\mathrm{BMO}(\mathbb{R})} \\
& \leq \sqrt{\pi}\left(\left\||D|^{1 / 2}\left(v^{0}(\cdot, t)-v_{T}\right)\right\|_{L^{2}(\mathbb{R})}+\left\||D|^{1 / 2}\left(w^{0}(\cdot, t)-w_{0}\right)\right\|_{L^{2}(\mathbb{R})}\right) \\
& =\sqrt{\pi}\left(\left\||D|^{1 / 2}\left(\int_{T}^{t} \partial_{s} P^{T-s} * v_{T} d s\right)\right\|_{L^{2}(\mathbb{R})}\right. \\
& \left.\quad+\left\||D|^{1 / 2}\left(\int_{0}^{t} \partial_{s} P^{s} * w_{0} d s\right)\right\|_{L^{2}(\mathbb{R})}\right) \\
& \leq \sqrt{\pi} T\left(\left|v_{T}\right|_{H^{3 / 2}(\mathbb{R})}+\left|w_{0}\right|_{H^{3 / 2}(\mathbb{R})} \leq 2 \sqrt{\pi} N T .\right.
\end{aligned}
$$

In the above we used Plancherel's theorem and the Fourier transform to estimate $V^{0}$ and $b^{0}$.

If

$$
0<T \leq T_{1}=\frac{m_{0}}{4 N M_{0} e^{8 N} \sqrt{\pi}},
$$

we have $\left\|b^{0}(\cdot, t)\right\|_{\mathrm{BMO}(\mathbb{R})} \leq m_{0} /\left(2 M_{0} e^{8 N}\right)$ for $t \in[0, T]$. From Lemma 7.2(i), we know $z^{0}=z^{0}(\alpha, t)$ is a chord-arc curve with

$$
\begin{aligned}
& \frac{1}{2} \frac{m_{0}}{e^{4 N}}|\alpha-\beta| \leq\left|z^{0}(\alpha, t)-z^{0}(\beta, t)\right| \leq\left(\frac{1}{2} \frac{m_{0}}{e^{4 N}}+e^{4 N} M_{0}\right)|\alpha-\beta| \\
& \quad \text { for } \alpha, \beta \in \mathbb{R}, t \in[0, T] .
\end{aligned}
$$

From now on, we assume $T$ satisfies (7.38). 
We construct a sequence of functions

$$
V^{j}=v^{j}+i w^{j} \in C\left([0, T], H^{3 / 2}(\mathbb{R})\right) \cap L^{2}\left([0, T], H^{2}(\mathbb{R})\right)
$$

by solving

$$
\left\{\begin{array}{l}
v_{t}^{j+1}-\mathfrak{a}^{j}|D| v^{j+1}=\mathfrak{f}_{1}^{j} \\
w_{t}^{j+1}+\mathfrak{a}^{j}|D| w^{j+1}=-\mathfrak{f}_{2}^{j} \\
v^{j+1}(\cdot, T)=v_{T}(\cdot), \quad w^{j+1}(\cdot, 0)=w_{0}(\cdot),
\end{array}\right.
$$

where $\mathfrak{a}^{j}=\mathfrak{a}\left(V^{j}\right)$ and $\mathcal{F}^{j}=\mathfrak{f}_{1}^{j}+i \mathfrak{f}_{2}^{j}=\mathcal{F}\left(V^{j}\right)$ are as defined in (7.3). We want to show that there is $0<T=T(N) \leq T_{1}$ such that for all $j \geq 0$,

$$
\begin{gathered}
\sup _{t \in[0, T]}\left|V^{j}(\cdot, t)\right|_{H^{3 / 2}(\mathbb{R})}^{2} \leq 8 N^{2}, \\
\int_{0}^{T} \int\left|\partial_{\alpha}^{2} V^{j}(\alpha, t)\right|^{2} d \alpha d t \leq 16 N^{2} e^{8 N},
\end{gathered}
$$

and $(1+i) \ln z_{\alpha}{ }^{j}(\alpha, t)=V^{j}(\alpha, t)$ defines a chord-arc curve $z^{j}=z^{j}(\alpha, t)$ for fixed $t \in[0, T]$ (with the freedom of choosing $z^{j}(0, t)$ ) such that

$$
\begin{aligned}
& \frac{m_{0}}{2 e^{6 N}}|\alpha-\beta| \leq\left|z^{j}(\alpha, t)-z^{j}(\beta, t)\right| \leq\left(\frac{m_{0}}{2 e^{6 N}}+e^{6 N} M_{0}\right)|\alpha-\beta| \\
& \text { for } \alpha, \beta \in \mathbb{R}, t \in[0, T] .
\end{aligned}
$$

We know $V^{0}$ satisfies (7.40)-(7.41). Assume that (7.40)-(7.41) holds for $V^{j}$. From (7.37) and (7.40),

$$
\mathfrak{a}^{j}=\frac{1}{2} e^{-\left(v^{j}+w^{j}\right)} \geq \frac{1}{2} e^{-8 N}=c_{0}
$$

So from Lemmas 7.3 and 7.7, $V^{j+1}$ exists in

$$
C\left([0, T], H^{3 / 2}(\mathbb{R})\right) \cap C^{1}\left([0, T], H^{1 / 2}(\mathbb{R})\right) \cap L^{2}\left([0, T], H^{2}(\mathbb{R})\right) .
$$

We want to show that (7.40)-(7.41) also holds for $V^{j+1}$ for some $0<T=T(N) \leq$ $T_{1}$. From Lemma 7.6, we have

$$
\begin{aligned}
\left|w^{j+1}(\cdot, t)\right|_{H^{3 / 2}(\mathbb{R})}^{2} & +c_{0} \int_{0}^{t} \int\left|\partial_{\alpha}^{2} w^{j+1}(\alpha, \tau)\right|^{2} d \alpha d \tau \\
& \leq e^{\kappa_{j} t}\left|w_{0}\right|_{H^{3 / 2}(\mathbb{R})}^{2}+\left(1+\frac{2}{c_{0}}\right) \int_{0}^{t} e^{\kappa_{j}(t-\tau)}\left|\mathfrak{f}_{2}^{j}(\cdot, \tau)\right|_{H^{1}(\mathbb{R})}^{2} d \tau
\end{aligned}
$$

and

$$
\begin{aligned}
& \left|v^{j+1}(\cdot, t)\right|_{H^{3 / 2}(\mathbb{R})}^{2}+c_{0} \int_{t}^{T} \int\left|\partial_{\alpha}^{2} v^{j+1}(\alpha, \tau)\right|^{2} d \alpha d \tau \\
& \quad \leq e^{\kappa_{j}(T-t)}\left|v_{T}\right|_{H^{3 / 2}(\mathbb{R})}^{2}+\left(1+\frac{2}{c_{0}}\right) \int_{t}^{T} e^{\kappa_{j}(\tau-t)}\left|\mathfrak{f}_{1}^{j}(\cdot, \tau)\right|_{H^{1}(\mathbb{R})}^{2} d \tau
\end{aligned}
$$


where $\kappa_{j}=c\left(\left\|\mathfrak{a}^{j}-1\right\|_{L^{\infty}\left([0, T], H^{3 / 2}(\mathbb{R})\right)}^{2}+1\right)$, and from (7.10) and (7.40), $\kappa_{j} \leq \kappa(N)$ for some constant $\kappa(N)$ depending on $N$. Therefore,

$$
\begin{aligned}
c_{0} \int_{0}^{T} \int\left|\partial_{\alpha}^{2} V^{j+1}(\alpha, t)\right|^{2} d \alpha d t \leq & e^{\kappa(N) T}\left(\left|w_{0}\right|_{H^{3 / 2}(\mathbb{R})}^{2}+\left|v_{T}\right|_{H^{3 / 2}(\mathbb{R})}^{2}\right) \\
& +\left(1+\frac{2}{c_{0}}\right) e^{\kappa(N) T} \int_{0}^{T}\left|\mathcal{F}^{j}(\cdot, t)\right|_{H^{1}(\mathbb{R})}^{2} d t
\end{aligned}
$$

Let

$$
T_{2}=\frac{\ln 2}{\kappa(N)} .
$$

Applying (7.11), (7.12), (7.40), and (7.42) to $\left|\mathcal{F}^{j}(\cdot, t)\right|_{H^{1}(\mathbb{R})}^{2}$, and taking $\varepsilon=$ $c_{0}^{2} /\left[8\left(c_{0}+2\right)\right]$ in (7.12), there is a constant $c_{1}(N)$ depending on $N$ such that for $0<T \leq \min \left\{T_{1}, T_{2}\right\}$,

$$
\begin{aligned}
\left(1+\frac{2}{c_{0}}\right) e^{\kappa(N) T} \int_{0}^{T}\left|\mathcal{F}^{j}(\cdot, t)\right|_{H^{1}(\mathbb{R})}^{2} d t & \\
& \leq c_{1}(N) T+\frac{1}{4} c_{0} \int_{0}^{T} \int\left|\partial_{\alpha}^{2} V^{j}(\alpha, t)\right|^{2} d \alpha d t .
\end{aligned}
$$

Let

$$
T_{3}=\frac{2 N^{2}}{c_{1}(N)}
$$

We obtain that for $0<T \leq \min \left\{T_{1}, T_{2}, T_{3}\right\}$,

$$
c_{0} \int_{0}^{T} \int\left|\partial_{\alpha}^{2} V^{j+1}(\alpha, t)\right|^{2} d \alpha d t \leq 8 N^{2}
$$

Moreover,

$$
\begin{aligned}
& \sup _{0 \leq t \leq T}\left|w^{j+1}(\cdot, t)\right|_{H^{3 / 2}(\mathbb{R})}^{2}+\sup _{0 \leq t \leq T}\left|w^{j+1}(\cdot, t)\right|_{H^{3 / 2}(\mathbb{R})}^{2} \\
& \quad \leq e^{\kappa(N) T}\left(\left|w_{0}\right|_{H^{3 / 2}(\mathbb{R})}^{2}+\left|v_{T}\right|_{H^{3 / 2}(\mathbb{R})}^{2}\right) \\
& \quad+\left(1+\frac{2}{c_{0}}\right) e^{\kappa(N) T} \int_{0}^{T}\left|\mathcal{F}^{j}(\cdot, t)\right|_{H^{1}(\mathbb{R})}^{2} d t \leq 8 N^{2} .
\end{aligned}
$$

This proves that for $0<T \leq \min \left\{T_{1}, T_{2}, T_{3}\right\}, V^{j+1}$ satisfies (7.40).

We now consider (7.41). We have

where

$$
\ln z_{\alpha}^{j+1}=\frac{1-i}{2}\left(v^{j+1}+i w^{j+1}\right)=\ln \xi_{\alpha}+b^{j+1},
$$

$$
\begin{aligned}
b^{j+1} & =\frac{1-i}{2}\left(\left(v^{j+1}-v_{T}\right)+i\left(w^{j+1}-w_{0}\right)\right) \\
& =\frac{1-i}{2}\left(-\int_{t}^{T} v_{\tau}^{j+1}(\cdot, \tau) d \tau+i \int_{0}^{t} w_{\tau}^{j+1}(\cdot, \tau) d \tau\right) .
\end{aligned}
$$


Therefore for $0 \leq t \leq T \leq \min \left\{T_{1}, T_{2}, T_{3}\right\}$,

$$
\begin{aligned}
& \| \operatorname{Re} b^{j+1}(\cdot, t) \|_{L^{\infty}(\mathbb{R})} \\
& \quad \leq \frac{1}{2}\left(\left\|v^{j+1}(\cdot, t)\right\|_{L^{\infty}}+\left\|v_{T}\right\|_{L^{\infty}}+\left\|w^{j+1}(\cdot, t)\right\|_{L^{\infty}}+\left\|w_{0}\right\|_{L^{\infty}}\right) \\
& \quad \leq\left|v^{j+1}(\cdot, t)\right|_{H^{3 / 2}(\mathbb{R})}+\left|v_{T}\right|_{H^{3 / 2}(\mathbb{R})}+\left|w^{j+1}(\cdot, t)\right|_{H^{3 / 2}(\mathbb{R})}+\left|w_{0}\right|_{H^{3 / 2}(\mathbb{R})} \\
& \quad \leq 6 N .
\end{aligned}
$$

On the other hand, we know from (7.39) that there is a constant $c(N)$ depending on $N$ such that for $0 \leq t \leq T \leq \min \left\{T_{1}, T_{2}, T_{3}\right\}$,

$$
\left|v_{t}^{j+1}(\cdot, t)\right|_{H^{1 / 2}(\mathbb{R})} \leq\left|\mathcal{F}^{j}(\cdot, t)\right|_{H^{1 / 2}(\mathbb{R})}+\left|\mathfrak{a}^{j}\right| D\left|v^{j+1}(\cdot, t)\right|_{H^{1 / 2}(\mathbb{R})} \leq c(N),
$$

and

$$
\left|w_{t}^{j+1}(\cdot, t)\right|_{H^{1 / 2}(\mathbb{R})} \leq\left|\mathcal{F}^{j}(\cdot, t)\right|_{H^{1 / 2}(\mathbb{R})}+\left|\mathfrak{a}^{j}\right| D\left|w^{j+1}(\cdot, t)\right|_{H^{1 / 2}(\mathbb{R})} \leq c(N) .
$$

Here we used Lemma 7.3 and Lemma 7.1(iii). Therefore

$$
\left\|b^{j+1}(\cdot, t)\right\|_{\mathrm{BMO}(\mathbb{R})} \leq \sqrt{2 \pi}\left\||D|^{1 / 2} b^{j+1}(\cdot, t)\right\|_{L^{2}(\mathbb{R})} \leq 2 \sqrt{\pi} T c(N) .
$$

Let

$$
T_{4}=\frac{m_{0}}{4 \sqrt{\pi} c(N) M_{0} e^{12 N}} .
$$

From Lemma 7.2(i), we have for fixed $t \in[0, T], 0<T \leq \min \left\{T_{1}, T_{2}, T_{3}, T_{4}\right\}$, that $z^{j+1}=z^{j+1}(\alpha, t)$ is a chord-arc curve, with

$$
\begin{aligned}
\frac{m_{0}}{2 e^{6 N}}|\alpha-\beta| & \leq\left|z^{j+1}(\alpha, t)-z^{j+1}(\beta, t)\right| \\
& \leq\left(\frac{m_{0}}{2 e^{6 N}}+e^{6 N} M_{0}\right)|\alpha-\beta| \quad \text { for } \alpha, \beta \in \mathbb{R} .
\end{aligned}
$$

This proves that $z^{j+1}$ satisfies (7.41). Therefore (7.40) and (7.41) hold uniformly for $\left\{V^{j}: j \geq 0\right\}$.

We now consider the convergence of the sequence $V^{j}$. From

$$
\left\{\begin{array}{l}
\left(v^{j+1}-v^{j}\right)_{t}-\mathfrak{a}^{j}|D|\left(v^{j+1}-v^{j}\right)=\mathfrak{f}_{1}^{j}-\mathfrak{f}_{1}^{j-1}+\left(\mathfrak{a}^{j}-\mathfrak{a}^{j-1}\right)|D| v^{j}=\tilde{f}_{1}^{j} \\
\left(w^{j+1}-w^{j}\right)_{t}+\mathfrak{a}^{j}|D|\left(w^{j+1}-w^{j}\right)=-\mathfrak{f}_{2}^{j}+\mathfrak{f}_{2}^{j-1}-\left(\mathfrak{a}^{j}-\mathfrak{a}^{j-1}\right)|D| w^{j}=\tilde{f}_{2}^{j} \\
\left(v^{j+1}-v^{j}\right)(\cdot, T)=0, \quad\left(w^{j+1}-w^{j}\right)(\cdot, 0)=0,
\end{array}\right.
$$

we apply Lemma 7.6. We have for $0 \leq t \leq \min \left\{T_{1}, T_{2}, T_{3}, T_{4}\right\}$,

$$
\begin{aligned}
& \left|\left(w^{j+1}-w^{j}\right)(\cdot, t)\right|_{H^{1 / 2}(\mathbb{R})}^{2}+c_{0} \int_{0}^{t} \int\left|\partial_{\alpha}\left(w^{j+1}-w^{j}\right)(\alpha, \tau)\right|^{2} d \alpha d \tau \\
& \quad \leq 2\left(1+\frac{2}{c_{0}}\right) \int_{0}^{t} \int\left|\tilde{f}_{2}^{j}(\alpha, \tau)\right|^{2} d \alpha d \tau,
\end{aligned}
$$


and

$$
\begin{aligned}
& \left|\left(v^{j+1}-v^{j}\right)(\cdot, t)\right|_{H^{1 / 2}(\mathbb{R})}^{2}+c_{0} \int_{t}^{T} \int\left|\partial_{\alpha}\left(v^{j+1}-v^{j}\right)(\alpha, \tau)\right|^{2} d \alpha d \tau \\
& \quad \leq 2\left(1+\frac{2}{c_{0}}\right) \int_{t}^{T} \int\left|\tilde{f}_{1}^{j}(\alpha, \tau)\right|^{2} d \alpha d \tau .
\end{aligned}
$$

Therefore for $0<T \leq \min \left\{T_{1}, T_{2}, T_{3}, T_{4}\right\}$,

$$
\begin{aligned}
& \sup _{[0, T]}\left|\left(v^{j+1}-v^{j}\right)(\cdot, t)\right|_{H^{1 / 2}(\mathbb{R})}^{2}+\sup _{[0, T]}\left|\left(w^{j+1}-w^{j}\right)(\cdot, t)\right|_{H^{1 / 2}(\mathbb{R})}^{2} \\
&+c_{0} \int_{0}^{T} \int\left|\partial_{\alpha}\left(V^{j+1}-V^{j}\right)(\alpha, \tau)\right|^{2} d \alpha d \tau \\
& \leq 4\left(1+\frac{2}{c_{0}}\right) \int_{0}^{T} \int\left|\left(\mathcal{F}^{j}-\mathcal{F}^{j-1}+\left(\mathfrak{a}^{j}-\mathfrak{a}^{j-1}\right)|D| V^{j}\right)(\alpha, \tau)\right|^{2} d \alpha d \tau \\
& \leq 8\left(1+\frac{2}{c_{0}}\right) \int_{0}^{T} \int\left|\left(\mathcal{F}^{j}-\mathcal{F}^{j-1}\right)(\alpha, \tau)\right|^{2} d \alpha d t \\
&+ 8\left(1+\frac{2}{c_{0}}\right)\left(\int_{0}^{T} \int\left|\left(\mathfrak{a}^{j}-\mathfrak{a}^{j-1}\right)\right|^{4} d \alpha d \tau\right)^{1 / 2} \\
& \cdot\left(\int_{0}^{T} \int|| D\left|V^{j}\right|^{4} d \alpha d \tau\right)^{1 / 2} \cdot
\end{aligned}
$$

In order to apply Lemma 7.4, we need to check that assumption (7.15) of Lemma 7.4 is satisfied. Let

$$
\begin{aligned}
\ln z_{\alpha}^{j}(\alpha, t ; \theta) & =\ln z_{\alpha}^{j}(\alpha, t)+\theta\left(\ln z_{\alpha}^{j+1}-\ln z_{\alpha}^{j}\right)(\alpha, t) \\
& =\ln \xi_{\alpha}(\alpha)+(1-\theta) b^{j}(\alpha, t)+\theta b^{j+1}(\alpha, t)
\end{aligned}
$$

where $b^{j}$ is as in (7.45). From (7.46) and (7.47) we know that

$$
\left\|\operatorname{Re}\left((1-\theta) b^{j}(\cdot, t)+\theta b^{j+1}(\cdot, t)\right)\right\|_{L^{\infty}(\mathbb{R})} \leq 6 N
$$

and

$$
\left\|(1-\theta) b^{j}(\cdot, t)+\theta b^{j+1}(\cdot, t)\right\|_{\mathrm{BMO}(\mathbb{R})} \leq 2 \sqrt{\pi} T c(N) .
$$

Therefore from Lemma 7.2(i), we know for $0 \leq t \leq \min \left\{T_{1}, T_{2}, T_{3}, T_{4}\right\}$, assumption (7.15) is satisfied, with $m=m_{0} /\left(2 e^{6 N}\right)$ and $M=m_{0} /\left(2 e^{6 N}\right)+e^{6 N} M_{0}$.

We now apply Lemma 7.4 to (7.49), taking $\varepsilon=c_{0}^{2} /\left(16\left(c_{0}+2\right)\right)$ in (7.17). We get, for some constant $c_{2}(N)$ depending on $N$,

$$
\begin{aligned}
& \sup _{[0, T]}\left|\left(v^{j+1}-v^{j}\right)(\cdot, t)\right|_{H^{1 / 2}(\mathbb{R})}^{2}+\sup _{[0, T]}\left|\left(w^{j+1}-w^{j}\right)(\cdot, t)\right|_{H^{1 / 2}(\mathbb{R})}^{2} \\
& \quad+c_{0} \int_{0}^{T} \int\left|\partial_{\alpha}\left(V^{j+1}-V^{j}\right)(\alpha, \tau)\right|^{2} d \alpha d \tau \\
& \quad \leq \frac{1}{2} c_{0} \int_{0}^{T} \int\left|\partial_{\alpha}\left(V^{j+1}-V^{j}\right)(\alpha, \tau)\right|^{2} d \alpha d \tau+c_{2}(N) T \sup _{[0, T]}\left|\left(V^{j}-V^{j-1}\right)(\cdot, t)\right|_{H^{1 / 2}(\mathbb{R})}^{2} .
\end{aligned}
$$


Let

$$
T_{5}=\frac{1}{2 c_{2}(N)} \quad \text { and } \quad T=\min \left\{T_{1}, T_{2}, T_{3}, T_{4}, T_{5}\right\}
$$

We obtain

$$
\begin{aligned}
& \sup _{[0, T]}\left|\left(V^{j+1}-V^{j}\right)(\cdot, t)\right|_{H^{1 / 2}(\mathbb{R})}^{2}+c_{0} \int_{0}^{T} \int\left|\partial_{\alpha}\left(V^{j+1}-V^{j}\right)(\alpha, \tau)\right|^{2} d \alpha d \tau \\
& \leq \frac{1}{2} c_{0} \int_{0}^{T} \int\left|\partial_{\alpha}\left(V^{j+1}-V^{j}\right)(\alpha, \tau)\right|^{2} d \alpha d \tau \\
& \quad+\frac{1}{2} \sup _{[0, T]}\left|\left(V^{j}-V^{j-1}\right)(\cdot, t)\right|_{H^{1 / 2}(\mathbb{R})}^{2} \cdot
\end{aligned}
$$

Therefore $V^{j}$ is a Cauchy sequence in $C\left([0, T], H^{1 / 2}(\mathbb{R})\right) \cap L^{2}\left([0, T], H^{1}(\mathbb{R})\right)$ and converges to a limit $V \in C\left([0, T], H^{1 / 2}(\mathbb{R})\right) \cap L^{2}\left([0, T], H^{1}(\mathbb{R})\right)$ as $j \rightarrow \infty$. Since $V^{j}$ is a bounded sequence in $C\left([0, T], H^{3 / 2}(\mathbb{R})\right) \cap L^{2}\left([0, T], H^{2}(\mathbb{R})\right)$, and $V$ is also the weak limit of $V^{j}$, we have

$$
V \in L^{\infty}\left([0, T], H^{3 / 2}(\mathbb{R})\right) \cap L^{2}\left([0, T], H^{2}(\mathbb{R})\right) .
$$

Furthermore, $z^{j}(\alpha, t)-z^{j}(0, t)$ is a Cauchy sequence in $C\left([0, T], H_{\mathrm{loc}}^{3 / 2}(\mathbb{R})\right)$ and converges to a limit $z(\alpha, t)-z(0, t)$ in $C\left([0, T], H_{\text {loc }}^{3 / 2}(\mathbb{R})\right)$. From (7.41), we know $z=z(\alpha, t)$ is chord-arc for each fixed $t \in[0, T]$ and satisfies

$$
\begin{aligned}
\frac{m_{0}}{2 e^{6 N}}|\alpha-\beta| & \leq|z(\alpha, t)-z(\beta, t)| \\
& \leq\left(\frac{m_{0}}{2 e^{6 N}}+e^{6 N} M_{0}\right)|\alpha-\beta| \quad \text { for } \alpha, \beta \in \mathbb{R}, t \in[0, T] .
\end{aligned}
$$

Moreover, $(1+i) \ln z_{\alpha}=V$.

From (7.39) we let $j \rightarrow \infty$ and apply Lemma 7.4. We conclude that $V=$ $v+i w$ is a solution of the quasi-linear system (7.5)-(7.3) satisfying data (7.34). Therefore $\bar{V}_{t}=\mathfrak{a}|D| V+\mathcal{F}$, or equivalently,

$$
\overline{\partial_{t} \ln z_{\alpha}}=\frac{1}{2 \pi i \bar{z}_{\alpha}(\alpha, t)} \int \frac{-z_{\alpha}(\alpha, t)+z_{\beta}(\beta, t)}{(z(\alpha, t)-z(\beta, t))^{2}} d \beta
$$

Notice that

$$
\int \frac{-z_{\alpha}(\alpha, t)+z_{\beta}(\beta, t)}{(z(\alpha, t)-z(\beta, t))^{2}} d \beta=\partial_{\alpha} \int \frac{1}{z(\alpha, t)-z(\beta, t)} d \beta .
$$

Since $\ln z_{\alpha} \in C\left([0, T], H^{1 / 2}(\mathbb{R})\right)$, using Lemma 7.2(ii), one may easily verify that

$$
\int \frac{1}{z(\alpha, t)-z(\beta, t)} d \beta=\int \frac{z_{\beta}(\beta, t)}{z(\alpha, t)-z(\beta, t)}\left(\frac{1}{z_{\beta}(\beta, t)}-1\right) d \beta
$$

is in $C\left([0, T], L^{2}(\mathbb{R})\right)$. Therefore $V=(1+i) \ln z_{\alpha} \in C^{1}\left([0, T], H^{-1}(\mathbb{R})\right)$. Using Lemma 7.6, we conclude that the solution

$$
V \in C\left([0, T], H^{3 / 2}(\mathbb{R})\right) \cap C^{1}\left([0, T], H^{1 / 2}(\mathbb{R})\right) \cap L^{2}\left([0, T], H^{2}(\mathbb{R})\right) .
$$


This proves Theorem 7.8.

Notice that (7.51) is equivalent to (1.6), since we may obtain (1.6) by multiplying $\bar{z}_{\alpha}$ on both sides of (7.51) and then integrating with respect to $\alpha$. Therefore a solution of the quasi-linear system (7.3)-(7.5) is also a solution of (1.6). This proves Theorem 1.4.

Acknowledgments. Financial support provided in part by National Science Foundation grants DMS-0100204 and DMS-0400643 and the Radcliffe fellowship.

Theorems 1.2, 1.4, and 1.5 were announced in [50]. I would like to thank the referee for going through this long paper and the many very helpful suggestions on improving the writing.

\section{Bibliography}

[1] Bergh, J.; Löfström, J. Interpolation spaces: an introduction. Grundlehren der Mathematischen Wissenschaften, 223. Springer, Berlin-New York, 1976.

[2] Birkhoff, G. Helmholtz and Taylor instability. Proc. Sympos. Appl. Math., Vol. XIII, 55-76. American Mathematical Society, Providence, R.I., 1962.

[3] Caflisch, R. E.; Orellana, O. F. Long time existence for a slightly perturbed vortex sheet. Comm. Pure Appl. Math. 39 (1986), no. 6, 807-838.

[4] Caflisch, R. E.; Orellana, O. F. Singular solutions and ill-posedness for the evolution of vortex sheets. SIAM J. Math. Anal. 20 (1989), no. 2, 293-307.

[5] Calderón, A.-P. Cauchy integrals on Lipschitz curves and related operators. Proc. Nat. Acad. Sci. U.S.A. 74 (1977), no. 4, 1324-1327.

[6] Chazarain, J.; Piriou, A. Introduction to the theory of linear partial differential equations. Studies in Mathematics and Its Applications, 14. North-Holland, Amsterdam-New York, 1982.

[7] Christ, M. Lectures on singular integral operators. CBMS Regional Conference Series in Mathematics, 77. American Mathematical Society, Providence, R.I., 1990.

[8] Coifman, R. R. Topics in nonlinear analysis. Lecture notes by M. Murray. Yale University, New Haven, Conn., 1982.

[9] Coifman, R. R.; Fefferman, C. Weighted norm inequalities for maximal functions and singular integrals. Studia Math. 51 (1974), 241-250.

[10] Coifman, R. R.; Jones, P. W.; Semmes, S. Two elementary proofs of the $L^{2}$ boundedness of Cauchy integrals on Lipschitz curves. J. Amer. Math. Soc. 2 (1989), no. 3, 553-564.

[11] Coifman, R. R.; McIntosh, A.; Meyer, Y. L'intégrale de Cauchy définit un opérateur borné sur $L^{2}$ pour les courbes lipschitziennes. Ann. of Math. (2) 116 (1982), no. 2, 361-387.

[12] Coifman, R.; Meyer, Y. Lavrentiev's curves and conformal mappings. Report no. 5. Institut Mittag-Leffler, Djursholm, Sweden, 1983.

[13] Coifman, R. R.; Meyer, Y. Nonlinear harmonic analysis, operator theory and P.D.E. Beijing lectures in harmonic analysis (Beijing, 1984), 3-45. Annals of Mathematics Studies, 112. Princeton University Press, Princeton, N.J., 1986.

[14] Coifman, R. R.; Rochberg, R.; Weiss, G. Factorization theorems for Hardy spaces in several variables. Ann. of Math. (2) 103 (1976), no. 3, 611-635.

[15] David, G. Thése de troisiéme cycle. Methématique. Doctoral dissertation, Université de Paris XI, 1981.

[16] David, G. Courbes corde-arc et espaces de Hardy généralisés. Ann. Inst. Fourier (Grenoble) 32 (1982), no. 3, xi, 227-239. 
[17] David, G. Opérateurs intégraux singuliers sur certaines courbes du plan complexe. Ann. Sci. École Norm. Sup. (4) 17 (1984), no. 1, 157-189.

[18] David, G.; Journé, J.-L. A boundedness criterion for generalized Calderón-Zygmund operators. Ann. of Math. (2) 120 (1984), no. 2, 371-397.

[19] David, G.; Journé, J.-L.; Semmes, S. Opérateurs de Calderón-Zygmund, fonctions paraaccrétives et interpolation. Rev. Mat. Iberoamericana 1 (1985), no. 4, 1-56.

[20] Delort, J.-M. Existence de nappes de tourbillon en dimension deux. J. Amer. Math. Soc. 4 (1991), no. 3, 553-586.

[21] DiPerna, R. J.; Majda, A. J. Concentrations in regularizations for 2-D incompressible flow. Comm. Pure Appl. Math. 40 (1987), no. 3, 301-345.

[22] Duchon, J.; Robert, R. Global vortex sheet solutions of Euler equations in the plane. J. Differential Equations 73 (1988), no. 2, 215-224.

[23] Ebin, D. G. Ill-posedness of the Rayleigh-Taylor and Helmholtz problems for incompressible fluids. Comm. Partial Differential Equations 13 (1988), no. 10, 1265-1295.

[24] Evans, L. C. Partial differential equations. Graduate Studies in Mathematics, 19. American Mathematical Society, Providence, R.I., 1998.

[25] Journé, J.-L. Calderón-Zygmund operators, pseudodifferential operators and the Cauchy integral of Calderón. Lecture Notes in Mathematics, 994. Springer, Berlin, 1983.

[26] Kambe, T. Spiral vortex solution of Birkhoff-Rott equation. Advances in fluid turbulence (Los Alamos, NM, 1988). Phys. D 37 (1989), no. 1-3, 463-473.

[27] Krasny, R. Desingularization of periodic vortex sheet roll-up. J. Comput. Phys. 65 (1986), 292313.

[28] Krasny, R. A study of singularity formation in a vortex sheet by the point-vortex approximation. J. Fluid Mech. 167 (1986), 65-93.

[29] Krasny, R. Computing vortex sheet motion. Proceedings of the International Congress of Mathematicians, Vol. I, II (Kyoto, 1990), 1573-1583. Mathematical Society of Japan, Tokyo, 1991.

[30] Ladyzhenskaya, O. A. Solution "in the large" of the nonstationary boundary value problem for the Navier-Stokes System with two space variables. Comm. Pure Appl. Math. 12 (1959), 427-433.

[31] Lebeau, G. Régularité du problème de Kelvin-Helmholtz pour l'équation d'Euler 2d. Séminaire: Équations aux Dérivées Partielles, 2000-2001, Exp. No. II, 12 pp. Séminaire: Équations aux Dérivées Partielles. École Polytechnique, Palaiseau, France, 2001.

[32] Liu, J.-G.; Xin, Z. P. Convergence of vortex methods for weak solutions to the 2-D Euler equations with vortex sheet data. Comm. Pure Appl. Math. 48 (1995), no. 6, 611-628.

[33] Lopes, M. C.; Lowengrub, J.; Nussenzveig Lopes, H. J.; Zheng, Y. X. Numerical evidence for nonuniqueness evolution for the 2D incompressible Euler equations: a vortex sheet example. Preprint, 2000.

[34] Lopes, M. C.; Nussenzveig Lopes, H. J.; Schochet, S. A criterion for the equivalence of the Birkhoff-Rott and Euler descriptions of vortex sheet evolution. arXiv: math. AP / 0502215. Preprint, 2005.

[35] Marchioro, C.; Pulvirenti, M. Mathematical theory of incompressible nonviscous fluids. Applied Mathematical Sciences, 96. Springer, New York, 1994.

[36] Meiron, D. I.; Baker, G. R.; Orszag, S. A. Analytic structure of vortex sheet dynamics. I. KelvinHelmholtz instability. J. Fluid Mech. 114 (1982), 283-298.

[37] Moore, D. W. The spontaneous appearance of a singularity in the shape of an evolving vortex sheet. Proc. Roy. Soc. London Ser. A 365 (1979), no. 1720, 105-119.

[38] Moore, D. W. Numerical and analytical aspects of Helmholtz instability. Theoretical and applied mechanics (Lyngby, 1984), 263-274. North-Holland, Amsterdam, 1985. 
[39] Pullin, D. I. The large-scale structure of unsteady self-similar rolled-up vortex sheets. J. Fluid Mech. 88 (1978), no. 3, 401-430.

[40] Pullin, D. I. On similarity flows containing two-branched vortex sheets. Mathematical aspects of vortex dynamics (Leesburg, VA, 1988), 97-106. SIAM, Philadelphia, 1989.

[41] Pullin, D. I.; Phillips, W. R. C. On a generalization of Kaden's problem. J. Fluid Mech. 104 (1981), 45-53.

[42] Rott, N. Diffraction of a weak shock with vortex generation. J. Fluid Mech. 1 (1956), 111-128.

[43] Saffman, P. G. Vortex dynamics. Cambridge Monographs on Mechanics and Applied Mathematics. Cambridge University Press, New York, 1992.

[44] Scheffer, V. An inviscid flow with compact support in space-time. J. Geom. Anal. 3 (1993), no. 4, 343-401.

[45] Shnirelman, A. On the nonuniqueness of weak solution of the Euler equation. Comm. Pure Appl. Math. 50 (1997), no. 12, 1261-1286.

[46] Stein, E. M. Singular integrals and differentiability properties of functions. Princeton Mathematical Series, 30. Princeton University Press, Princeton, N.J., 1970.

[47] Sulem, C.; Sulem, P.-L.; Bardos, C.; Frisch, U. Finite time analyticity for the two- and threedimensional Kelvin-Helmholtz instability. Comm. Math. Phys. 80 (1981), no. 4, 485-516.

[48] Van Dyke, M. An album of fluid motion. Parabolic Press, Stanford, Calif., 1982.

[49] Vishik, M. Incompressible flows of an ideal fluid with vorticity in borderline spaces of Besov type. Ann. Sci. École Norm. Sup. (4) 32 (1999), no. 6, 769-812.

[50] Wu, S. Recent progress in mathematical analysis of vortex sheets. Proceedings of the International Congress of Mathematicians, Vol. III (Beijing, 2002), 233-242. Higher Education Press, Beijing, 2002.

[51] Yudovich, V. Non-stationary flow of an ideal incompressible liquid. Comp. Math. Math. Phys. 3 (1963), 1407-1457.

[52] Yudovich, V. I. Uniqueness theorem for the basic nonstationary problem in the dynamics of an ideal incompressible fluid. Math. Res. Lett. 2 (1995), no. 1, 27-38.

\section{SiJUE WU}

Department of Mathematics

University of Michigan

2844 East Hall

530 Church Street

Ann Arbor, MI 48109

Email: si jue@umich.edu

Received December 2004. 